Fune 1, 1900. Species from T.du Z. East - $\begin{array}{r}1019 \\ 1067\end{array}$

Auy 17,1405 
Freshet in Baddecter hianga. Sas, ayil6 - masheremay kidge, patatatoficers r ruen monimp machinex Bugr graull thit at Beaverone, C.B. moith Exploing 



\section{FLORA OF MOUNT DESERT.}


BR = Bonaventure River.

Bar = Barachois de Malkaie

Breg = Belgium.

Carl = Caletion

LC= hanotaseaperia

$c_{a}=$ Cacouna $\quad$ In $\alpha=$ mont Louis

$D T=$ Douglastorm $R$. mHa = mialbat

$D=$ Douflostorn $\quad R S_{A}=R_{1}$ Stedmedeatwonto

Da TDarte: Dattmonth Staum: " $T="$

Dach - Dalhousie

$y p=$ your thice

Coc $=$ Cocuminac

FP = Father Paint

In: LaspéPaím

$u_{R}=$ haw Pines

\&C. Lista Cascaperia

mall = malbail

Thex = hetapeitia

$r=$ howalle

$M R=$ truthichnond

Rest = Restigonahe R

$T T=$ Table Trp

Trae $=$ Tracahigarh

$y k=y$ yok 
$C=$ Cutcer, Thaise

$n_{1}=$ Machias."

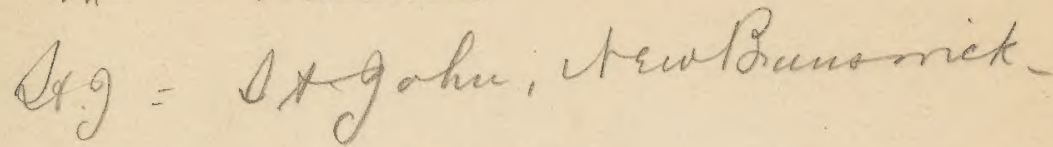

$\beta=$ Bathwet, " "

NC. = Haw Cacile, Quchec

$P=$ Paspébice.

$P D=$ PartDanis.

Pur $=$ Pointr Hauvelle -

$S h=$ Shigamake

$B R=$ Bonaventure River

Rdud = Riviere du Laup

$Q=$ Quiber $r$ sububs to Silleny $r$ t montruorcue:

$C_{a}=$ Cacouma -

$S=$ tyiney bapelpeton

$W=$ Vinioor (halfhousistade) hava Scatio

$t=$ tentwille

SP = SFarre Point

SM.: Aheffics nills

NR= Vaulk Paad, wouth mt.

$R A=$ Blacte Wole near Baytersttaber

Reat = Trectignehe R. at metaperia 


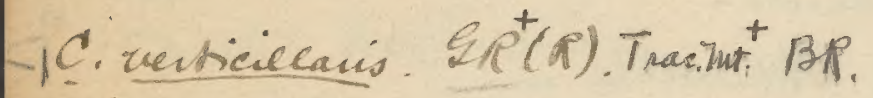

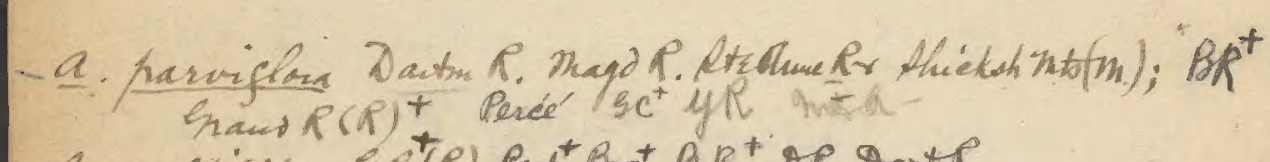

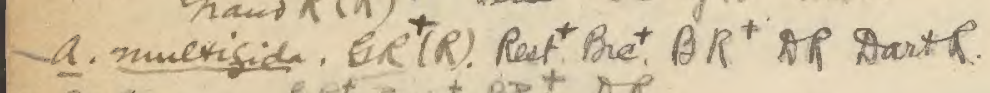

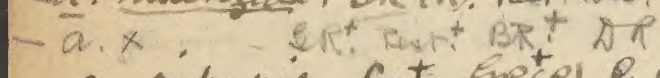

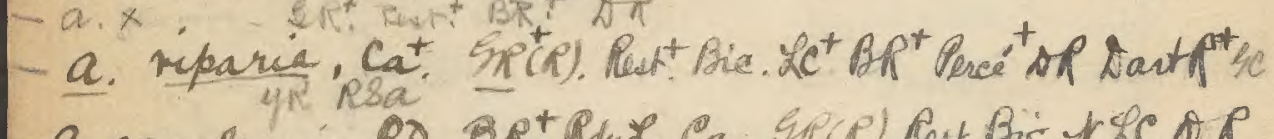

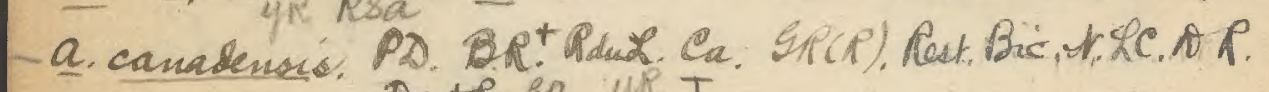
DaitR, PC YRT

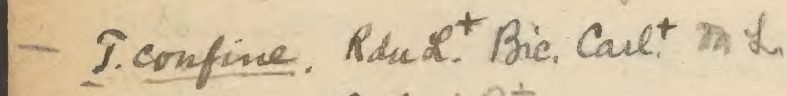

- T. alpinum Espt ypt

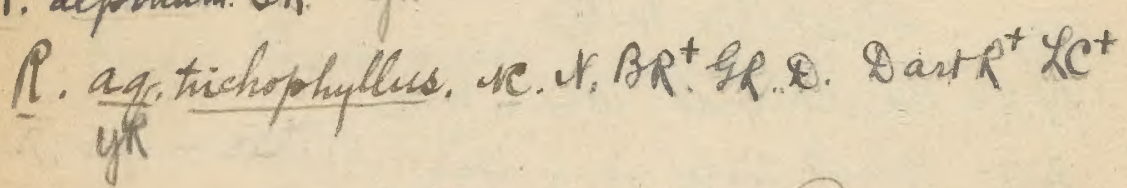

- 1 . Purshï. BR. Burtons, M. Trecr. Canbolange, 


\section{CATALOGUE OF PLANTS.}

Series I. PHANEROGAMIA; FLOWERING PLANTS.

Class I. DiCOTyledones ANGIOSPERMEA.

Division I. POLYPETALA.

\section{RANUNCULACEA. Crowfoot Famitr.}

CLEMATIS, L. Virain's Bower.

I. C. Virginiana, L.

Thickets; infrequent. Wasgatt Brook; mouth of Hadlock $\mathrm{G}$. th Brook (J. L. Wakefield); - Northeast Harbor, etc. (Rand); - M P. $\mathcal{K}$. Duck Brook (Rand, F. M. Day); - on Doctors Brook (R. \& R.); - Echo Notch (Redfield).

\section{A. mesnorose, $\mathrm{L}$.}

ANEMONE, L. WIND-FLOWER.

Rare; apparently occurring only in the southeastern part of the Island. Otter Creek (Grace H. Eliot); - near Schooner Head (Clara L. Walley, Mary Minot).

THALICTRUM, L. Meadow Rue.

T. polygamum, Muhl. T. Cornuti, Man., 5th ed., non L.*C B. Whe,

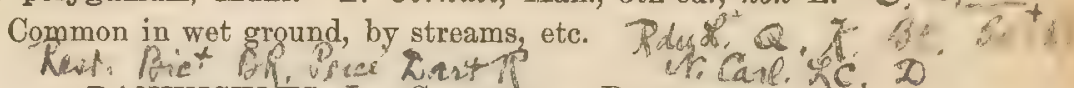
RANUNCULÚS, L. Crowfoot. ButTercup.

R. Cymbalaria, Pursh. Seaside Crowfoot.

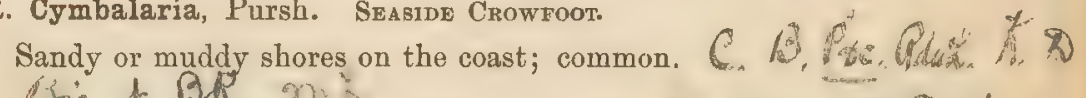
He. A. PR. See Trelease in Bot. Gaz., xi. 92. Dast R. Yt. 
5 R. Flammula, L., var. reptans (L.), E. Meyer. Crempina SPEARW ORT.

Gravelly shores; infrequent. Pool near Schooner Head (Red-

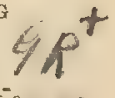
field, G. Hunt); - shores of Great Pond; Ripples Pond (Rand).

R. abortivus, L. Small-fiowered Crowfoot.

Grassy fields; rare. Southwest Harbor; Somesville; Bar p, Af, Harbor (Rand). Probably introdyced in grass seed.

R. recurvatus, Poir. Theur. XC. Peree lacth.

Rare. Hadlock Valley (Redfield). of gC $^{+}$

8 R. repens, L. SṔtTED-LEAF Buttercup. $h /$

C. St \% Frequent in moist ground by roadsides, in meadows, etc. While in some places, as by roadsides, this species is doubtB. Ae. Q.S. less introduced, it appears indigenous in others. It is not L. Situ ( $m$ so abundant on the Island as to lead to any strong presumpRoutmer, Stytion of its general introduction, or of its spreading to some remote places. If . Caif atP. TRP. Pence, D. Dait $R$.

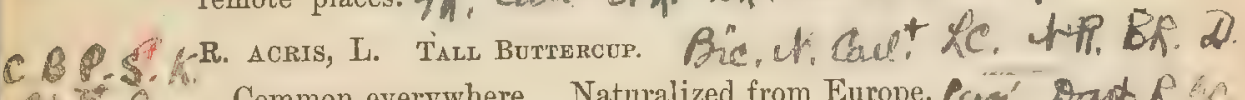
fis. Common everywhere. Naturalized from Europe. Pcece thach $f$. $F$

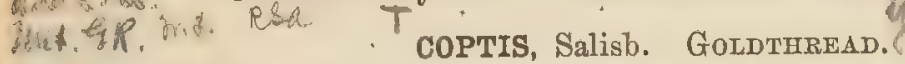

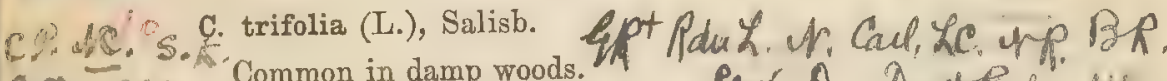
smp. PC. "Common in damp woods.

\section{AQUILEGIA, L. Columbine.}

\section{A. Canadensis, L.}

Q

Rare. High Head (Rand); - East Point, Seal Harbor (A. Räe Cope).

a. vulgaris, L. Garjen Columbine. Carl.

C. $f$ Occasional by roadsides and in waste places. Escaped from Bie, H $_{\text {: }}$ abunsizear of ficultivation. Near Jordan Pond (Rand); - near Ovens (Green- $2 B$, leaf, Lane \& Rand) ; - Town Hill (R. \& R.).

\section{ACT ÆEA, L. BANEBERRY.}

( 3 A. alba (L.), Miller.

Deep woods; infrequent. Sargent Mt. Gorge, etc. (Rand); Hadlock Valley (Redfield); - near Bar Harbor (F. M. Day). 


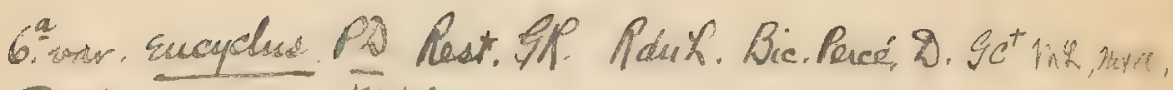
R. sur inta

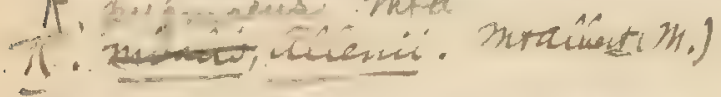

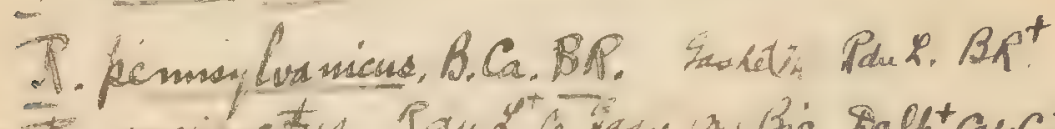

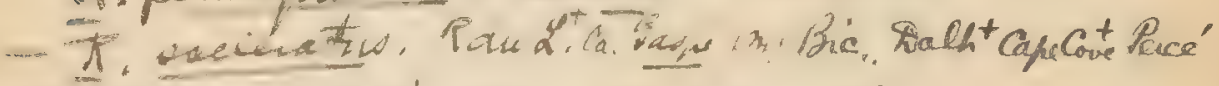

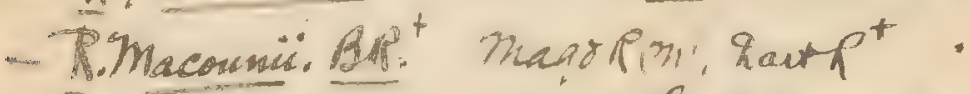

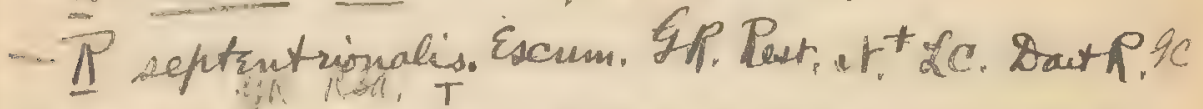

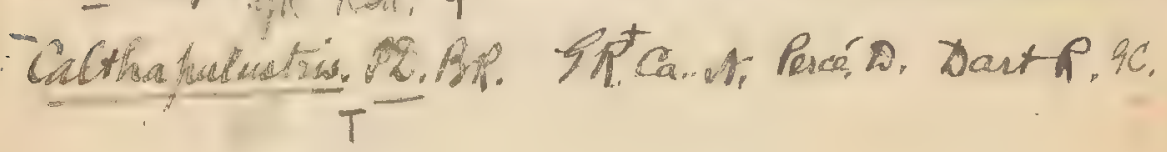

Aconitumetrpetfres. Q.

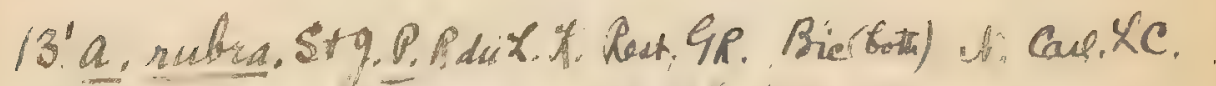

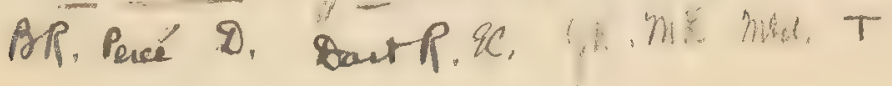


t. rabuodiseum to.

14. minimana metazer $\left(x_{1}\right)$.

P. varigatum Rest. $N^{+}$ER.

6. whenese Riet 


\section{BERBERIDACEAE. Barberry Family.}

BERBERIS, L. BARBERRY.

\section{B. VULGaris, L. Commox Barberry.}

Escaped from gardens, or rarely spontaneous. Roadside north of Seal Harbor, - this station now destroyed (Rand, Redfield); clearing, Canada Valley (Rand); - roadside near Norwood Core (Rand, Annie S. Downs); - Ox Hill, Seal Harbor (Redfield).

\section{NYMPHAEACEAE。 WATER-LILY FAMILT.}

BRASENIA, Schreb. WATER ShIELd.

IJ Beltata (Thunb.), Pursh.

Abundant in Witch Hole (Rand, Redfield, F. M. Day); - MR. Somes Pond (R. \& R., M. I. Fernald); - Ripples Pond (Rand).

\section{NYMPHAEA, L. WATER-LILY.}

N. odorata, Ait.

Common in ponds and meadow streams. A form with very $/ \mathcal{C}, S$, small flowers, Mountain Pond, Sargent Mit. (Rand).

NUPHAR, Smith. Cow-lily. Spatter Dock. Yellow POND-LILY.

N. advena, Ait. $f$.

Common in ponds, slow streams, and bog boles. C. vre.

SARRACENIACE

Sarracenia, L. Pitcher-piant. Side-saddle Flower.

S. purpurea, L.

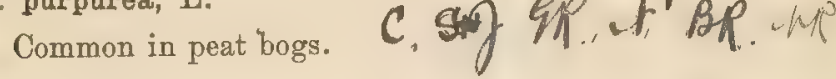

\section{FUMARTACE E. Fumitory Family.}

CORYDALIS, Vent.

\section{C. glauca (Mœnch), Pursh.}

Rocky ground and burnt clearings; frequent, but nowhere in $C, \mathcal{B}$.

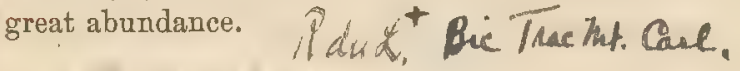
pis linati 
FUMARIA, L. Fumitory.

20 F. OFFICINALis, L.

Waste ground, Great Cramberry Isle (R. \& R.). Adventive from Europe.

CRUCIFER ZE. Mustard Family.

CARdamine, L: Bitter Cress.

21 C. hirsuta, L.

Frequent in brooks and on pond shores. So far as known the Island plants are all glabrous, and in other respects do not correspond to the typical European plant. Whether our common American plant is not specifically distinct seems to be an open question. That it is so distinct, see N. L. Britton, Bull. Torr. Bot. Club, xix. 219. As, however, there appear to be intermediate forms, perhaps it would be wiser to give it ouly varietal rank. The Mt. Desert forms can perhaps be classified under the three following heads for convenience, although apparently there are no well defined dividing lines between them.

P. P2. T. (a) Forma Pennsylvanica. C. Pennsylvanica, Mubl. GlaRkú. pedicels somewhat divergent. Roadside ditch between Town Hill and Northwest Cove; near outlet of Great Pond; Infervale Brook (Rand).

$(\beta)$ Like the last, but with widely divergent pedicels, and Bipici Heck D. Daith ac. thicker, much shorter pods. The most common form. Brook, $T$ Clark Valley; Cold Brook; Intervale Brook (Rand); Doctors Brook; Stanley Brook (Redfield);-Deer Brook (R. \& R.).

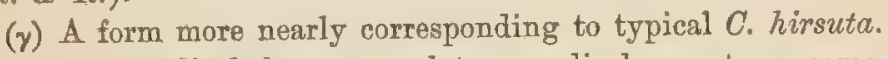
Glabrous; radical leaves rosulate; pedicels erect or somewhat spreading; style short and stout; pods variable in length and thickness. Shores of Northwest Arm, Great Pond (Rand, M. L. Fernald).

22 C. parviflora, L.

Leaflets mostly linear; radical leaves few or none; pods linear, erect on spreading pedicels. In dry ground, or among moist rocks; rare. Little Duck Island; Flying Mt. (Rand). 


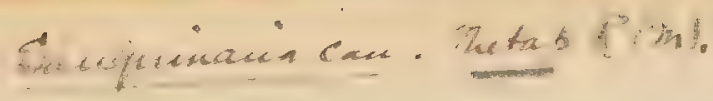

Panaw Pracas Nou L. Q. cul,

3. Hommiferum Q. CapeCove.

chelidonicus majus. Q.

C. pratanois BR Sout th

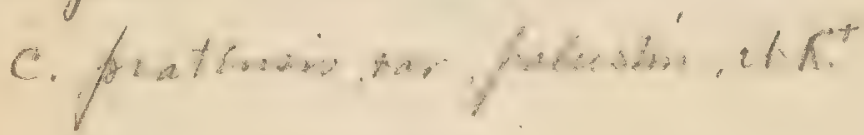

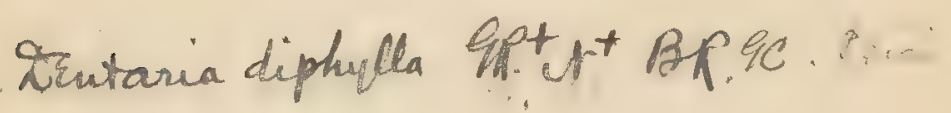




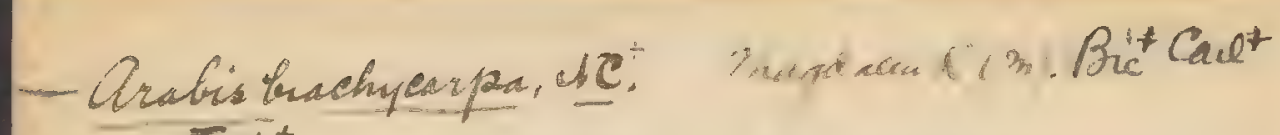
$\mathrm{Tad}^{+}$

- Arcomease. Hidu $\mathbb{Z}^{+}$

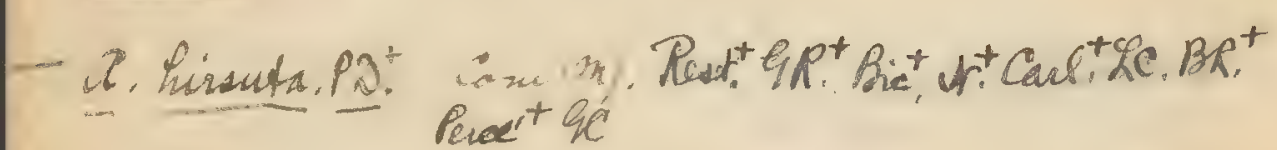

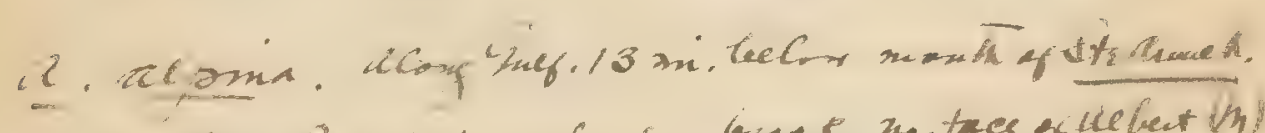

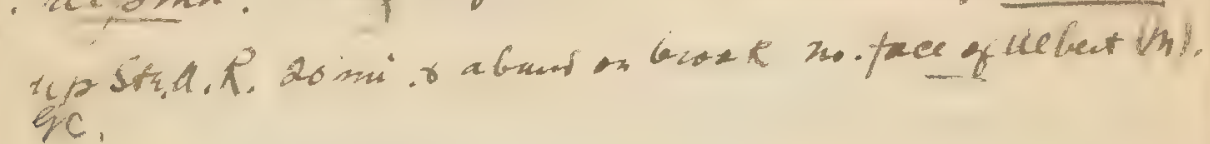

- A. Colinsï. Bie 
Frabr nugaspensa $\mathcal{P}^{+}$

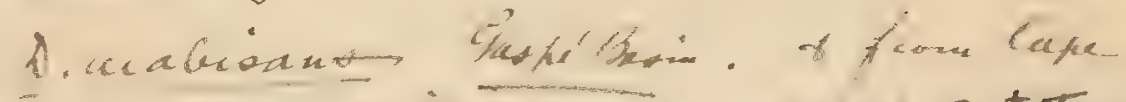

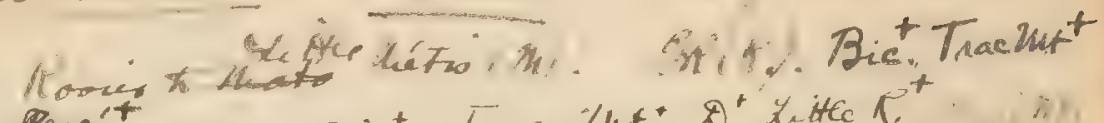

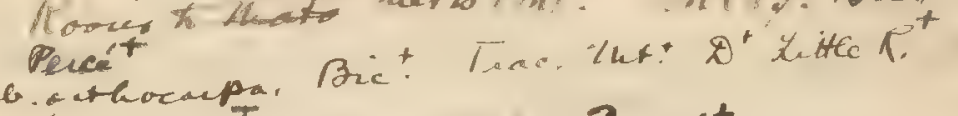

Rancenthocupa

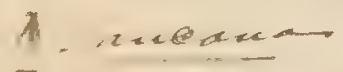

ion (hit: Beceét

D. micaur, confusa Perac't

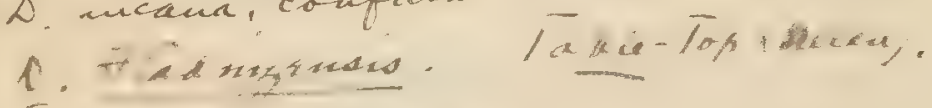

D. hucnoapenas Precét

7. RAtala:s Bices

D. Garatis Biet 
3. thethe 14

Heopecionationsija, sor.

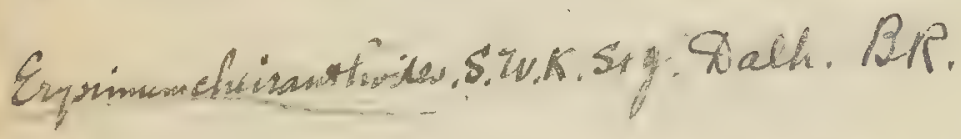

\$. Sofrica duation month of thagd. $112 \mathrm{~m}$.

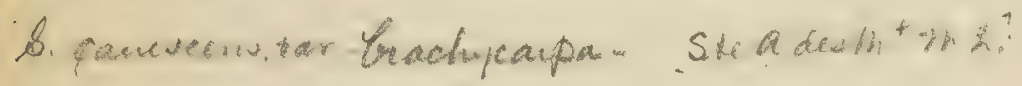

B. juneen. Q. S. Nor.

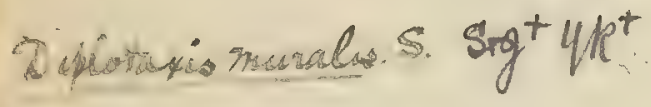

Diplot.srucoides YKt

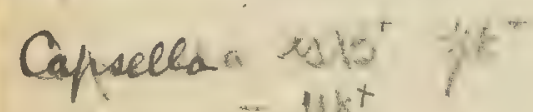
$-4 k^{t}$ 
NASTURTIUM, R. Br. WATER CRESS.

23 N. palustre (Leys.), DC. Marsh Cress.

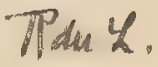

Rare. Field, Somesville (Rand). Doubtless introduced.

24 N. Armoracta (L.), Fries. Horseradish.

Rare. Escaped from cultivation to waste places. Somesville $p$, Gie (Rand). Adventive from Europe.

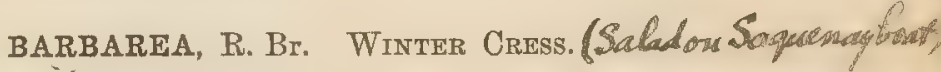

2.5 . 5 . vulgaris, R. Br. , Yellow Rocket.

Rare. Wayside, Bar Harbor (Rand). Lately introduced. Q Woutest

SISYMBRIUM, L. Hedge Mustaró.

S. officinale (L.), Scop. Common Hedge Mustard.

Roadsides and waste places. Naturalized from Europe. N. S.M.

\section{BRASSICA, L.}

2 B. Sinapistrum, Boiss. Charlock.

Old fields and waste places; infrequent. Northeast Harbor; Southwest Harbor; Somesville (Rand). Adventive from C. B. $2 . Q$

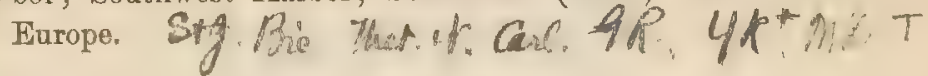

Z 8 B. migra (L.), Koch. Brack Mostard,

Old fields and waste places; frequent. Northeast Harbor; $\$ W_{s} \&$. High Head; beach, Greening Island; Southwest Harbor (Rand). Adventive from Europe.

2 B. CAMPESTRTS, I. TURNiP.

Old fields and waste places; frequent. Somesville (R. \& W. ?. R.); - Seal Harbor (Redfield); - Southwest Harbor; Northeast Harbor (Rand). Introduced from Europe.

CAPSella, Medic. Shepherd's Purse.

C. Bursa-pastoris (L.), Monch. Mi K

A common weed, - even at Duck Islands (Redfield). Natura1 C. B. Q Q.S.

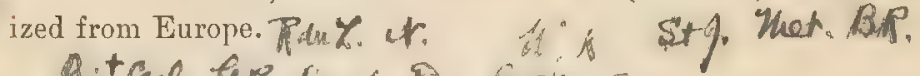

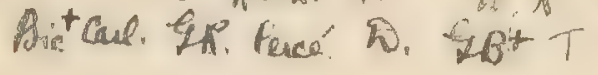




\section{LEPIDIUM, I. Peppergrass.}

3 L. Virginicum, L.

Roadsides; rare. Southwest Harbor (M. L. Fernald);- Somesville (Rand); - Bar Harbor (W. H. Manning). Recently introduced from the South.

CAKILE, Gærtn. SEA Rocket.

J2 c. Americana, Nutt.

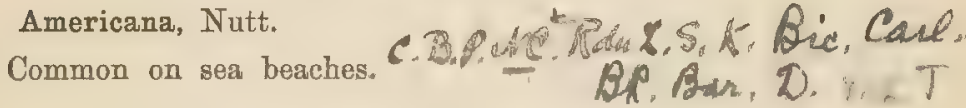

RAPHANUS, L. RADISH.

33 R. Raphanistrum, L. Wild Radish, Jojmted Charlock.

Old fields and waste places; frequent. Northeast Harbor $C$. B.P. (W. H. Dunbar); - Southwest Harbor; Beech Hill; Somesville; 5 Bar Harbor, etc. (Rand). Adventive from Europe.Ceul: QR,

CISTACE $\not$ E. ROCK-Rose Famitr.

\section{HUDSONIA, L.}

34 H. ericoides, L. Heath-Like Hedsonta.

Frequent on mountain summits. Also on borders of Sea Wall Swamp, and on Bass Harbor road (Annie S. Downs).

LECHEA, L. PinWeEd.

35 L. minor, L., var.

Very common in dry soil. The form found on the Island corresponds to $L$. intermedia, Leggett MS. $=L$. Leggettii, Britt. \& C \& Holl., var. intermedia (Legg.), Britt. \& Holl.,-according to Dr. N. L. Britton.

VIOLACEAE. VIOLET Family.

VIOLA, L. VIOLET.

36 . palmata, I. cucullata (Ait.), Gray.

Common, mostly in moist ground. Very variable. Forma albiflora.

Flowers pure white. Occasional. Emery District (Wm. C. Lane). 


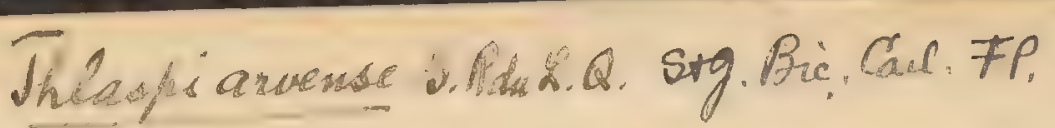

Canchia sativa $4 k^{+}$

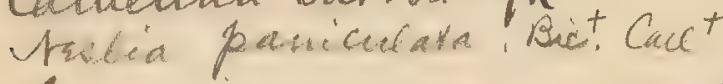

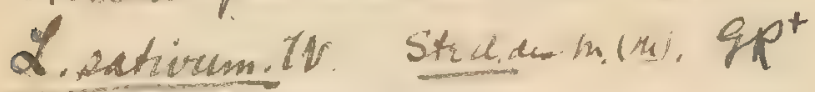

X. Apetalum. Iv. Sig.

L. ruderale.

t. eampesta. Tr.

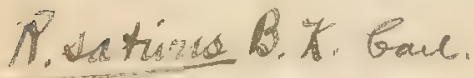

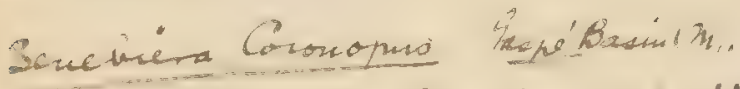

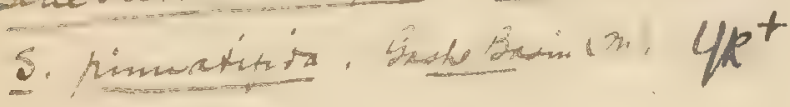

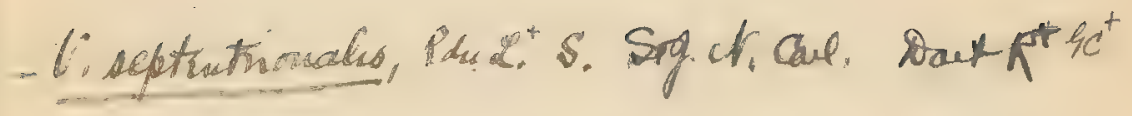

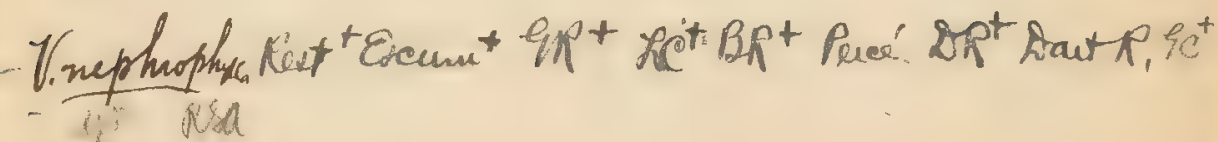


V. Fimbriatula. F.

V. paluotins. Acherp(ation).

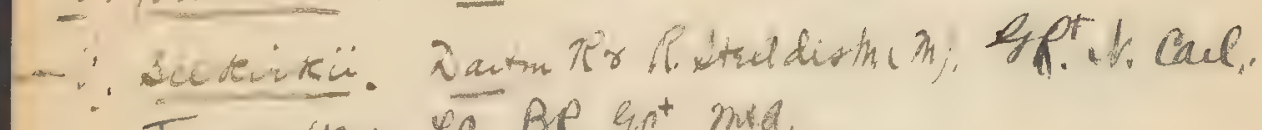

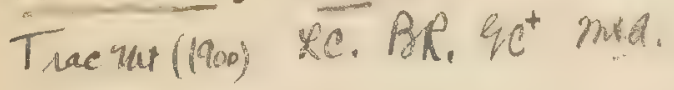

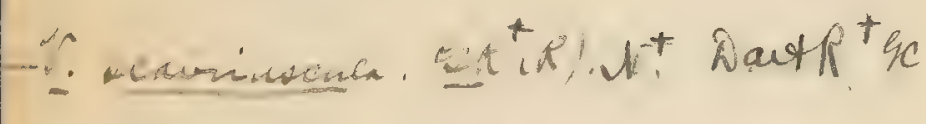

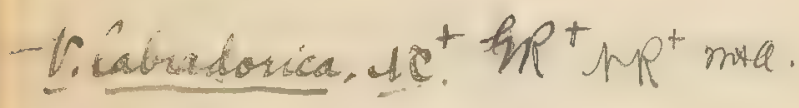

V. admed, caut 


\section{Forma variegata.}

Flowers blue, mottled with white. Occasional. Southwest Harbor; Somesville (Rand).

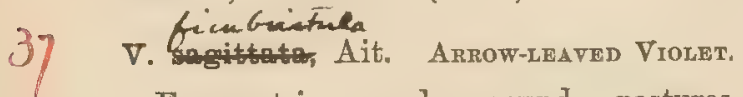

Frequent in open dry ground,-pastures and hillsides.

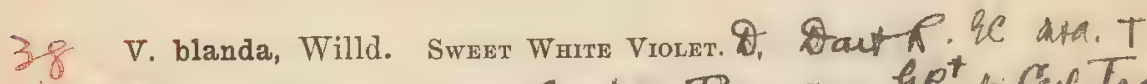

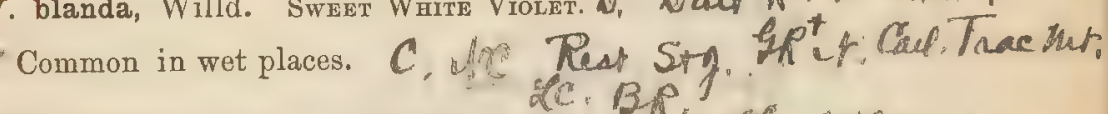

Fenifolia, Gray. Kidnet-tigated Vrolet.

Q.

Occasional. Southwest Harbor (Greenleaf, Lane \& Rand); -

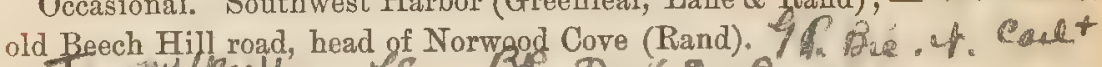

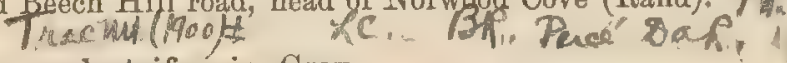

Var. Mulustrifoming matar.

Not uncommon in mossy ground. Seal Harbor (Redfield); Southwest Harbor (M. L. Fernald). antal:

4/ V. primulafolia, L. Primrose-Leaved Violet.

Infrequent. Southwest Harbor (Greenleaf, Lane \& Rand, M. L. Fernald); - old road to Beech Hill, head of Norwood Cove; shore of Pond Heath (Rand).

2 V. lanceolata, L. Larce-Leaved Vrolet.

Common in wet places and roadside ditches. $C$.

comeperea

V. animo, fro, Mor Mublanhergii (Torr.), Gray. DOG VIOLET.

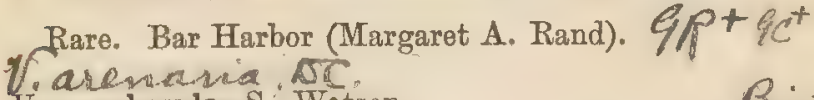

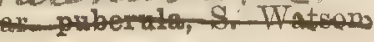

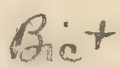

Frequent in dry soil. High Head; pasture near Pond Heath; Northwest Cove (Rand); - Bar Harbor (Mary Minot).

V. tricolor, L. Pangy. Heart's-ease, Ladigs' Delight.

Escaped from cultivation. Bar Harbor (W. H. Manning). Adrentive from Europe. 


\section{CARYOPHYLLACE $E$.}

DIANTHUS, L. Pink.

D. deltoides, L. Maiden Pink.

Well established in field, Bar Harbor (Mary Minot); - field, Northeast Harbor (B. E. J. Gresham). Adventive from Europe.

SAPONARIA, L.

4 S. VACCARia, L. Cow Herb.

Uncommon. By roadside, Town Hill; in old grain field, Southwest Harbor (Rand). Adventive from Europe.

silene, L, Catchrly. Campion.

S. NoCtiflora, L. Night-FLowering CATChFly. Occasional in waste places. Roadside near Sargent Cove; Lefg, $_{\text {, }}$
Somesville; Southwest Harbor (Rand). Adventive from Europe.

S. Armeria, L. Sweet William Catchfly.

Occasionally escaped from gardens to roadsides and waste places. Southwest Harbor; Sea Wall; between Fernald and Norwood Coves (Rand). Adventive from Europe.

S. Cucubalus, Wibel. S. inflata, Smith. Buader Campron. \& Well established in field, Bar Harbor (Mary Minot). Ad- $6 \neq \mathrm{dC}$, ventive from Europe nur. Bie. Cou de BR. Pereé Relus

S. NUTANS, $\mathbf{L}$.

Well established in field, Bar Harbor, although not abundant (Mary Minot). Adventive from Europe.

LYCHNIS, L. COCKLE.

S 2 L. vespertina, Sibth. Evening Lychis. White Campion.

Rare. Waste ground, Fernald Point (Rand). Adventive from Europe.

L. Githago (L.), Scop. Conn Cockle.

Fields and roadsides; occasional. Southwest Harbor; North- C $\ell . T$. east Harbor (Rand); - Bracy Cove (Wm. C. Lane); - Seal Harbor (Redfield). Adventive from Europe. 
i. Gatuin. RSA

S. officinalis. Q.S.K.

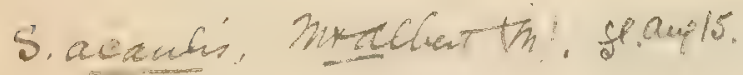

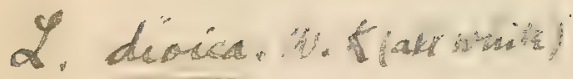

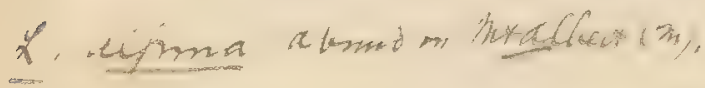




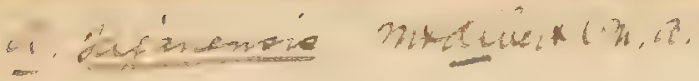

1. serpellifolis. K.

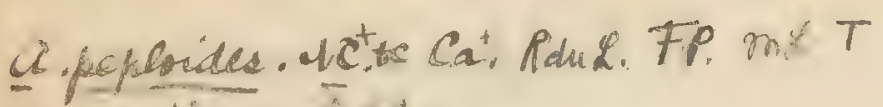

A. arctica 7rth-

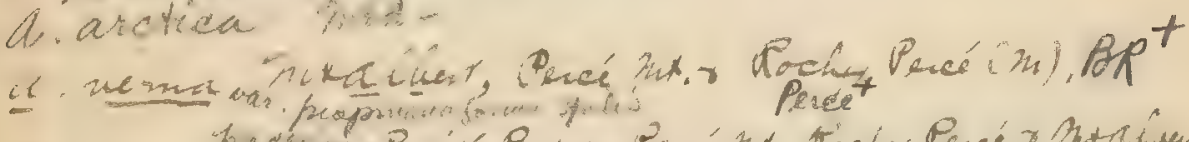

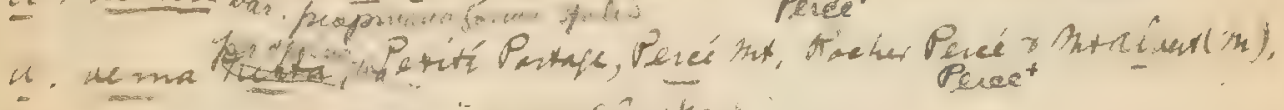

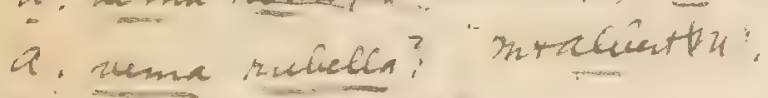

a. Eitore Core"

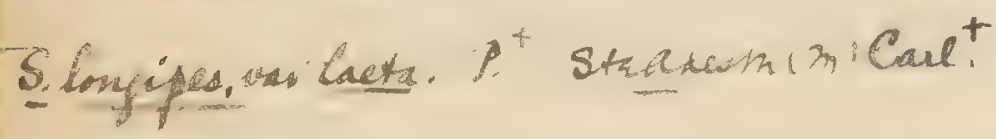

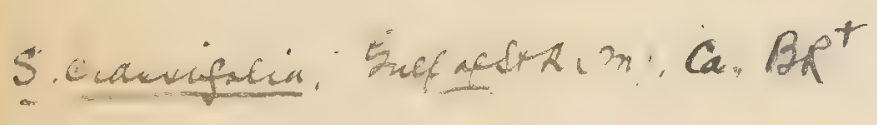


ARENARIA, L. SANDWORT.

\section{A. Grœnlandica (Retz), Spreng.}

Frequent on mountain summits; often on rocky hills of less altitude; and less frequently on headlands and rocky shores by the sea, as at Bar Harbor (Rand). At Mt. Desert this plant blooms throughout the entire season from early June to October, the later flowers, however, being much smaller in size and fewer in number.

\section{A. laterifiora, I. $\mathbb{D} T$}

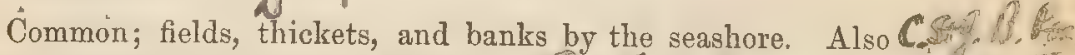
Duck Islands (Elizabeth G. Britton). Ridis. S. S. Sucum . Buc Caut

STELLARIA, 'L. CHIOKWEED. STARWORT.

S. media (Ls.), Smith. Commos Chuckwerd,

Common in cultivated and waste grounds. Naturalized from C., O O S Europe. Bic, or Caul BA, IR, Peré, IB.T K.

\section{S. longifolia, Muhl. Long-LEAVEd STarwort.}

Rare. Damp ground on Intervale Brook, near Hulls Cove (R. \& R.).

\section{S. GRAminea, L. English Starwort,}

Becoming frequent in grassy places. Bass Harbor road, near C. B. $A \%$. Southwest Harbor; Southwest Harbor; Northwest Cove; Town Hill (Rand); - Northeast Harbor (Redfield). Adventive from

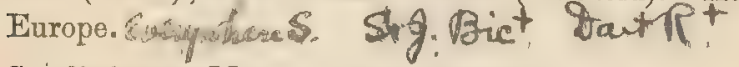

S. uliginosa, Murr. Swamp Starwort.

Rare. In wet ground and roadside ditch, east side of North-S. east Harbor (T. Meehan \& Redfield).

S. borealis, Bigel. Northern Starwort. Daut R, G if

nuta. Frequent in wet ground. Little Harbor Brook Notch, Somesville (Rand); - Long Pond meadows (Wm. C. Lane); - Green Mt.; Bear Island (Redfield); - Otter Creek (T. G. White). Cue. IR.

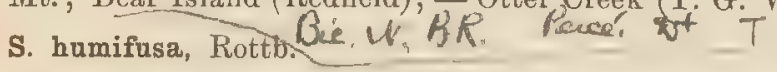

Rare. Salt marsh, Little Cranberry Isle (Redfield). There $C, B, C$ is only one other station for this plant within the limits of Gray's Manual, but it is common farther north.
Bic $\mathrm{H}^{+} \mathrm{Cal}$. AR
DartR. 


\section{CERASTIUM, L. MOUSE-EAR CHICKWEED.}

62 c. vULGatum, L. Gray, Manual, 6th ed. C. viscosum of D.PS Pda ?

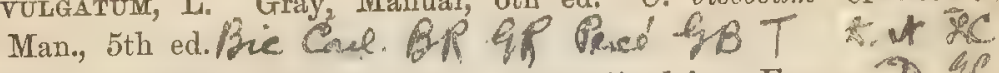
Fields and waste places; common. Naturalized from Europe. $D$ GC

63 c. arvense, L. Firld Chickweed. Rare. Duck Islauds (Rand, Redfield, Annie S. Downs); 7 F.
field near Ship Harbor (Redfield \& Faxon).

SAGINA, L. PearLWORT.

\section{$6 \%$ s. procumbens, $\mathrm{L}$.}

Springy places and wet rocks; frequent. Sea Wall (H. C.C. $\$$ fof B. B

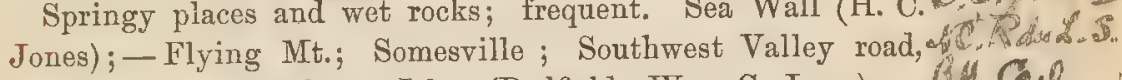
etc. (Rand); - Cranberry Isles (Redfield, Wm. C. Lane); BH Col. Duck Islands (Redfield); - Great Head (F. M. Day). Bre. Bif. SAP.

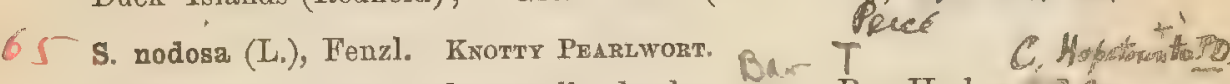
Crevices of rocks and gravelly banks; rare. Bar Harbor/R.e.

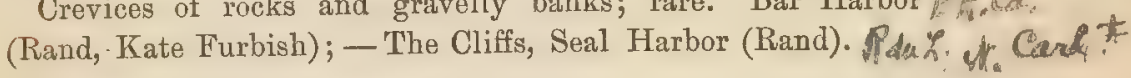

BUDA, Adans. (Spergularia, Presl.) Sand SpURReY.

B. rubra (L.), Dumort. Spergularia rubra (L.), Presl. PIs

Sayd Spurrey.

Frequent; dry sandy soil, and occasionally on sea beaches. $\$ . \mathbb{*}$. Manchester Point, Northeast Harbor; Bar Harbor (Rand); $-\mathcal{E} B^{+} y K^{+}$
Seal Harbor; Northeast Harbor; Little Cranberry Isle (Redfield); - Great Cranberry Isle; Fernald Point (R. \& R.); - on beach, Great Cranberry Isle (Rand). This plant in dry soil is strictly procumbent, ustrally undersized and dwarfed; on beaches it becomes large and widely spreading.

B. marina (L.), Dumort. Spergularia salina, Presl. T

Sea beaches; rare. Sutton Island; Great Cranberry Isle Wr font (Rand). Gaul it on Rat Bie

B. borealis, S. Watson. Spergularia borealis (S. Watson),

Robinson. matrue

Frequent on sea beaches and salt marshes. Wasgatt Cove $C$ C P $P$ dus (J. L. Wakefield); - Somesville; Little Cranberry Tsle (Red- SP" Caret +

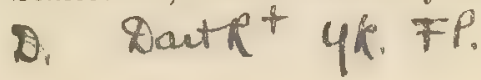




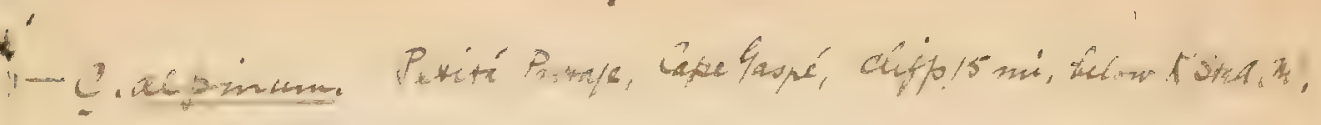

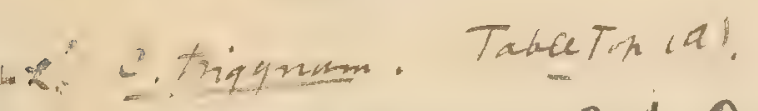

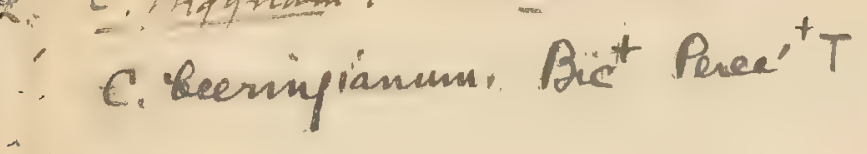

\&......

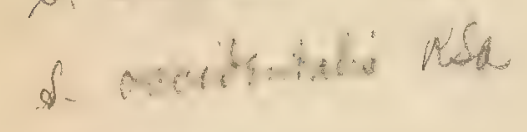

659 and the and 

field); - Southwest Harbor; Sea Wall; Norwood Cove; High Head; Bar Harbor, etc. (Rand); - Great Cranberry Isle (R. \& R.).

\section{SPERGULA, L. SPURREY.}

S. ARvensis, I. Corn Spurrey.

A common weed in cultivated ground. Adrentive from $C, P_{0} P_{1}$ Europe. M. Caul. Mf. Puec' D. yft Bre.T Forma RUBRA.

Flowers deep pink. Field, Salisbury Cove (Rand).

\section{PORTUlaCACE A. Purslane Family.}

MONTIA, L. BLinks.

Sepals 2, ovate, persistent, herbaceous. Petals 5, united at base, 3 somewhat smaller. Stamens 3 , rarely more, on the tube of the corolla. Ovary free, 3-ovuled: style 3-cleft, very short. Capsule 3-valved, 3-seeded. Seeds black, dull, tuberculate, rarely smoothish or shining.-A small branching glabrous succulent annual, with opposite leaves, and small axillary or racemose flowers. Bot. Cal., i. 77.

\section{M. fontana, $\mathrm{L}$.}

Stems procumbent or ascending, 1 to 3 inches long: leaves spatulate to linear oblanceolate, 3 to 9 lines long: flowers a line long or less: capsule globose. (Bot, Cal., l. c.) Rare. Damp, brackish ground, Great Cranberry Isle (Rand); - Great Duck Island (Redfield). The only stations thus far known within the limits of Gray's Manual, or in Eastern U. S. Common, however, farther north, and on Pacific shores. Dat $\mathrm{f}^{+}$
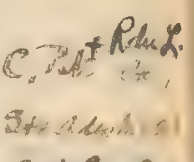
Bpt Capene

\section{Portulaca, L. Purslane.}

P. oleracea, L. Purslane. Purslet.

Cultivated and waste grounds. Bar Harbor (Rand, W. H. * . Manning); - Long Pond (Rand). As yet a very uncommon weed on the Island, and probably introduced since 1880. Naturalized from Europe. 


\section{ELATINACE E W Waterwort Family.}

ELATINE, L. WATERWORT.

\section{E. Americana (Pursh), Arn.}

Rare. Margin of Somes Stream and of Mill Pond, Somesville (Rand, M. I. Fernald).

\section{HYPERICACE E. ST. JoHn's-wort FAmiLY.}

HYPERICUM, L. ST. JOHN'S-WORT.

\section{* H. adpressum, Bart.}

Rare. Adventive from farther south. Along the railroad, Green Mountain (Arnold Greene).

H. ellipticum, Hook.

Wet places and bogs; frequent. Pond Heath; Northeast C. B. Harbor; Southwest Harbor; High Head, etc. (Rand); - Somesville (R. \& R.); - Squid Cove (Redfield).

H. PERforatum, L. Common St. John's-wort.

Fields and roadsides; frequent in some parts of the Island. B. RAu A. Q.S. A well known weed. Naturalized from Europe.

\section{H. mutilum, L.}

Frequent in low grounds and wet places. Outlet of Great Pond; Southwest Harbor; Somesville, etc. (Rand); - shores of Great Pond (Redfield).

\section{H. Canadense, L.}

Common in wet, sandy soil. C.S. \&. Srof Var. majus, Gray.

Frequent. Southwest Harbor; Northeast Harbor; Denning Brook; Long Pond meadows; Somesville; shores of Jordan Pond and Great Pond; Bass Harbor, etc. (Rand); - Seal Harbor; Little Cranberry Isle (Redfield).

Two somewhat peculiar forms of this variety are sometimes met with on the Island:- 
76: Goveale. C.B.S.

5. 
$T$ ins resticilata.

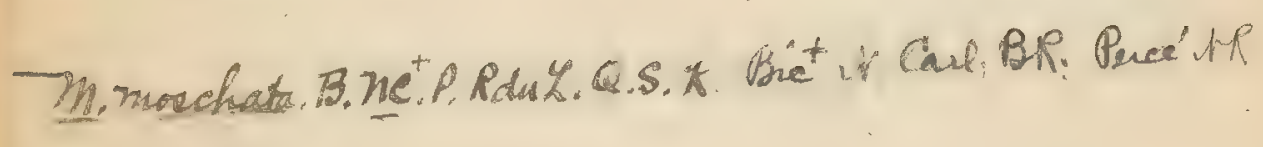

Th. enista. A.

Tilin ansineres $Q$

2 costhaticas. 
(a) Simple; leaves ascending and somewhat appressed. Southwest Harbor, etc. (Rand).

$(\beta)$ Leaves broadly lanceolate, more or less strongly fivenerved at the base. Intervale Brook; Southwest Harbor, etc. (Rand); - shores of Jordan Pond (Redfield).

Y H. nudicaule, Walt. H. Sarothra, Mx. Prne Weed. Oranae Grass.

Sandy or gravelly soil, roadsides and mountain tops; common. The mountain form is exceedingly dwarfed, often being no more than a single unbranched stem, less than one inch in height.

ELODES, Adans. MARSH St. Jomr's-wort.

E. campanulata (Walt.), Pursh. E. Virginica (L.), Nutt. ' $\mathrm{D}^{+} . \mathrm{H}$ '

Common in swamps and bogs, and on borders of ponds. C.B. A C Tdue

MaLVACE $A$. Maliow Family.

MALVA, L. MaLLOW.

M. Rotundifolia, L. Common Mallow. Cheeses.

Waste and cultivated grounds about dwellings; not very com- $\mathbb{Z}$. mon. Naturalized from Europe.

w.k. $4 k$.

2 M. ALCÈA, L.

Escaped to roadsides between Town Hill and Salisbury Cove (M. L. Fernald). Adventive from Europe.

LINACE F. Flax Family.

LINUM, L. FLAX.

83 L. usitatissimum, L. Common Flax.

Uncommon. Roadsides near Seal Cove, and about Southwest Po, Q, Harbor; abundant in grain field, Southwest Harbor (Rand). We, care. Adventive from Europe. $-213$. 


\section{GERANIACE ES. Geranium Familu. \\ GERANIUM, L.}

\section{G. Robertianum, I. Hers RoBert.}

Frequent in damp, rocky places, especially at head of sea $f:$ beaches. Abundant on Cranberry Isles (R. \& R., F. M. Percet Day);-Flying Mt. (Rand); - Bald Porcupine Island (W. H. Manning).

\section{G. Carolinianum, L.}

Frequent in waste places and clearings. Southwest Harbor (Harriet A. Hill); - roadside near Denning Pond (Annie S. Downs); - Somesville; Town Hill; Hulls Cove; High Head; Bubble Pond, etc. (Rand); - Seal Harbor (Redfield); - Bar Harbor (W. H. Manning).

OXALIS, L. WOOD SORREL.

\section{Acetosella, L.}

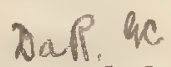

Peuce 0.

Common in mossy ground, deep cold woods. This plant is believed to be the true Irish Shamrock, although the emblem is $5.5 \mathrm{Ac}$

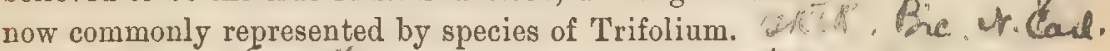

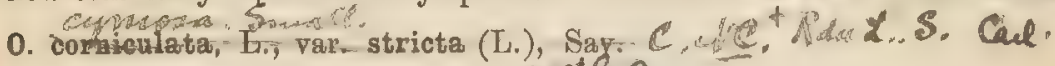
Common, mostly in open ground. BP. IR,

\section{IMPATIENS, L. BALSAM. JEWEL WeEd,}

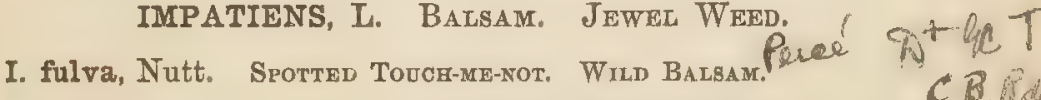
Moist places; common. A spurless form, Sea Wall (Rand). Forma albitora. Daf, Phe Cas. PR. IR.

Flowers white or cream-color, spotted with pink; stems and foliage very pale. Southwest Harbor (Rand).

\section{ILICINE AE. Holly Family.}

ILEX, L. HOLLY.

I. verticillata (L.), Gray. Black Alder.

Low grounds and thickets; common. 5 , f 
pattuse. 1xitions.

Qf fuenaicusn, Q

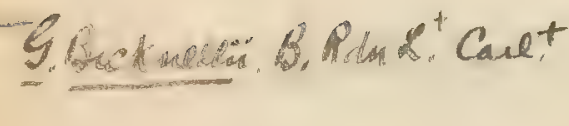

Erotium cicutariom, Cail FP.

E.moochatam, $4 k^{+}$

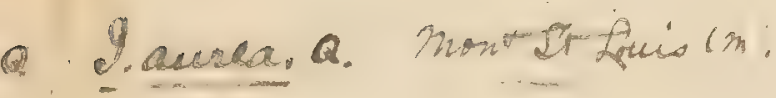




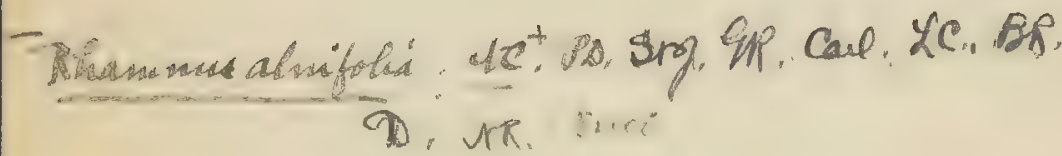

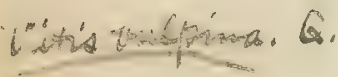

var, tactorasm, Q. 
Var. tenuifolia, Torr.

Leaves petiolate, obovate, thin, smooth beneath except a slight pubescence on the midrib, uncinately serrate, obtuse, or more commonly mucronate-tipped; pistillate flowers 4-5-cleft, commonly solitary, short-pedicelled; berries scarlet. A shrub about $5^{\circ}$ high with very slender branches. Torr. Fl. North. States, 338. A woodland form, appearing most distinct from the type.* On Denning Brook, Somesville (M. L. Fernald).

NEMOPANTHES, Raf. Mountain Holdy.

N. fascicularis, Raf. N. Canadenis (Mx.), DC.C.S.S. E. S F P

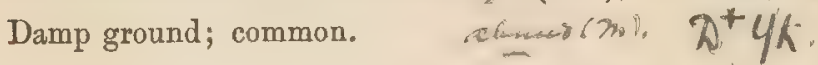

\section{VITACE}

AMPELOPSIS, MX.

A. quinquefolia (L.), Mx. Virgrnian Creeper. Woodbrne.

Common in cultivation, and often escaped to roadsides and waste places. Squid Cove; Southwest Harbor; Somesville (Rand). There is no satisfactory evidence that this plant is indigenous on the Island. Introduced from farther south.

SAPINDACE A. SoApberRy Family.

ACER, L. MAPLE.

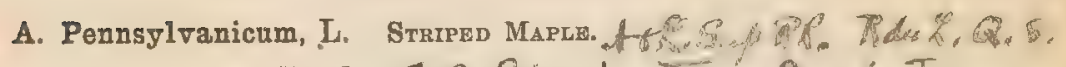

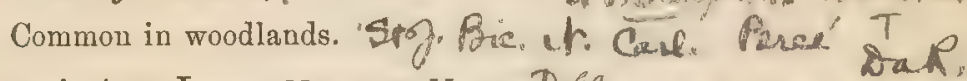
A. spicatum, Lam. Mountain Maple. D. $/$ C.

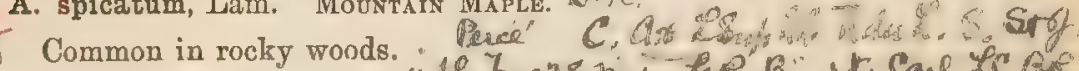

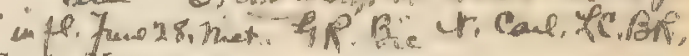
A. saccharinum, Wang. SugAr d LAPLE. $T$ Infrequent. Sargent District, etc. (Rand); Seal Harbor, of etc. (Redfield); - near Bar Harbor (W. H. Manning). Q. S. K. W.

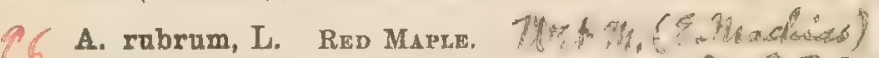
Common in swamps and damp ground C. B P.QS S. T. O. Coul.

* See also Britton in Bull. Torr. Bot. Club, xvii. 314. 


\section{ANACARDIACE E. Cashew Family.}

\section{RHUS, L. SUMach.}

q. R. typhina, T. Staghorn Sumach.

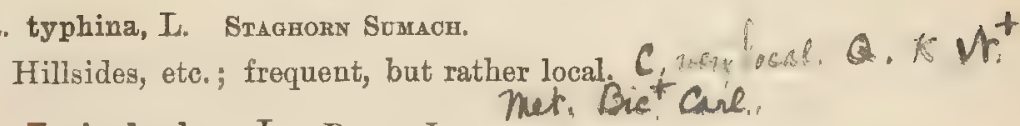

R. Toxicodendron, L. PoIson Ivr,

Thickets and low grounds; frequent, but local in its distri- $\mathbf{Q}$. Ther, bution. Common on cliffs and rocky banks by the seashore; - $W_{\text {i }}$ Pierce Head; Little Harbor; Hunters Beach; Roberts Point, Trae mar (1900) etc. (R. \& R.); - especially on the southern shore 'of the Island. It is also found in some abundance about Somesville and vicinity in low grounds and by roadsides. Poisonous to the touch.

\section{POL YGalace a. Mrlikwort Family.}

POLYGALA, L. MiLKWORT.

\section{P. paucifolia, Willd. Frisged Polygala.}

Infrequent and local. Reported by various collectors from $/ \mathrm{Rp}^{+}$ different parts of the region south and east of Salisbury Cove. Also found southwest of Youngs District (Clara L. Walley, Greenleaf, Lane \& Rand).

P. sanguinea, $\mathrm{L}$.

Infrequent. Fields, Bar Harbor; Southwest Harbor (Rand); Long Pond meadows (Redfield); - "Mt. Desert" (R. H. Day).

P. verticillata, $\mathrm{L}$.

Rare. Fields, Norwood Road, Southwest Harbor (Rand, Anna H. Bee).

ILGUMinOSA. Pulse Faminy.

TRIFOLIUM, L. ClOVER. TrefoIl.

102 T. ARVENSE, T. RABBIT-Foot Clover.

Old fields, roadsides, etc.; common. Naturalized from Europe. 7. 



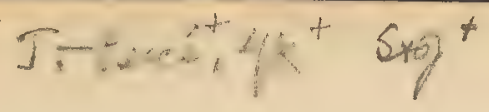

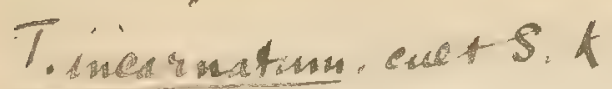

$9-y k$ men

Latus corniculatus, s. 
$\ell^{3}$ T. PRAThNse, I., RED Clover. T

Fields and pastyres; common. Naturalized from Europe. C.T3, Rdos,

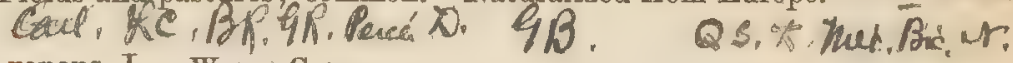

T. repens, L. Whitr Clover.

Common everywhere in fields, pastures, and by waysides.C.,$P$, Naturalized froy Europe, and possibly jndigenous northward. TAd $\mathscr{A}, \mathbb{S}, \ldots$

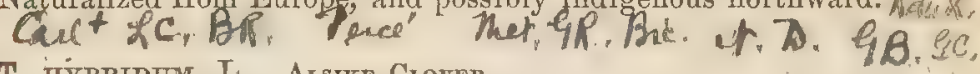

fof T. HÝrRIDUM, L. Alsike Crover.

Roadsides and fields; becoming common. This beautiful 73 P, 48 clover was rare on the Island ten or twelve years ago. Since $\mathbf{Q}, \overline{\$}, \bar{x}_{5}$ that time it has appeared in increasing abundance every year. Ther. 9 p.er. It does not seem, however, to be cultivated, or introduced inten che.. tionally. Naturalized from Europe. $4 \mathrm{c}, 4 / 8$,

T. AGRARIUM, L. Hop CLOVER.

Infrequent in fields and by roadsides. Southwest Harbor 5 , 3 m. (Rand, Harriet A. Hill); - Seal Harbor (Redfield);-Bar

- Harbor (Mary Minot); - Beech Hill (Rand). Adventive from Europe.

\section{T. Procumbens, L, Low Hop Clover.}

Common in fields and by roadsides. Naturalized from Europe, but appearing indigenous. Small, simple, erect forms ( $A_{m} \mathrm{Am}_{\mathrm{A}} \mathrm{B}_{\mathrm{a}}$ ) are common. This is "the real Irish Shamrock" of the newspapers, a long account of which appears regularly every two or three years. The error has been exposed so many times that it seems almost needless to refer to it here. (See Oxalis Acetusella, page 88. )

\section{MELILOTUS, Juss. Mellot.}

108 M. offictraits (L.), Willd.

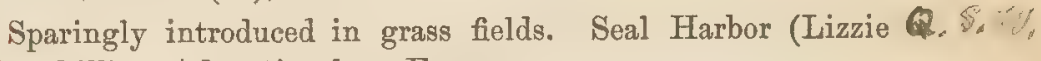
Churchill). Adventive from Europe.

M. ALBA, Lam.

By waysides and in waste ground; more common than the $B$ last. Goose Cove (Wm. C. Lane); - Bar Harbor (F. M. Caret FP. 
Day); - Somesville (Annie S. Downs); - Seal Harbor (Red field, Lizzie Churchill); - Eden; Fermald Point (Raud). Adventive from Europe.

\section{MEDICAG0, L。 MEDICK.}

M. LUpUlina, L. Buack Medick. Smaxts.

Sparingly introduced. Beach, Sea Wall (Rand); - Bar Har S. D. Q . bor (Mary Minot). Adventive from Europe. BR, W , K, Met, Ca.e

ROBINIA, L. LOCUST-TKE.

R. Pseudacacia, L. Common Locust.

Escaped from cultivation to roadsides. Southwest Harbor; Q Somesville; Town Hill, etc. (Rand); - Seal Harbor (Redfield). Adventive from the widdle.States.

\section{R. viscosa, Vent. Clammy Locust.}

Escaped from cultivation. 'Rog̨dside, Northeast Harbor (Red- wh. field). Adventive from the mountains of the Southern States.

Desmodium, Dest. TiçK Treforl.

D. acuminatum (Mx.), DC.

Rare. Clearing, Northwest Arm woods, Great Pond (Annie S. Downs). Perhaps introduced, as this species has not as yet been found elsewhere on "the island.

\section{VICIA, L. Vetch. TARE.}

V: sativa, L. Comron Vetoh, or Tare. Pincé

Common, especially on sea beaches. Naturalized from Europe.

A very pubescent form with flowers often peduncled, Bar Har- Bic, wh bor (Mary Minot).

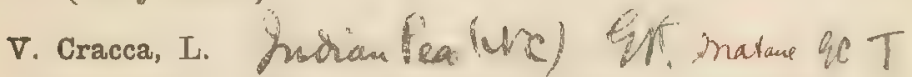

Frequent in fields. Southwest Harbor; Somesville, etc. C.S4\%. 19 (Rand); - Northeast Harbor (R. \& R.); - Seal ILarbor (Sara T \& \& \&, R. E. Boggs, Redfield); - Town Hill (Faxon \& Rand); - Bar Har- Reas Gic. Cast. bor (Mary Minot, W. H. Manning). LC. of. Pr. Pees

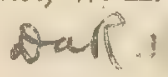




$$
\text { med- } 1 k^{+}
$$

$\because$ M.sethro.B.S.

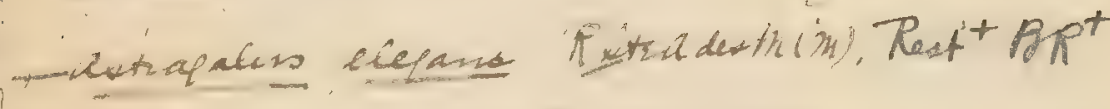

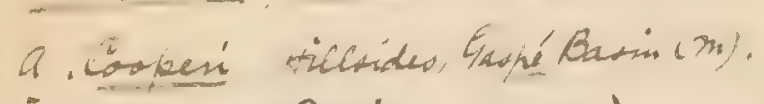

- A. alpinus, Reot (monewarta)

a. figioses, var, americamus, $\mathrm{KC}^{+} \mathrm{BP}+$

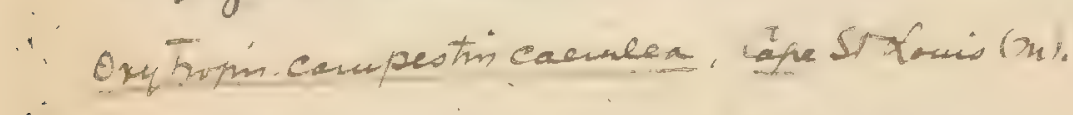

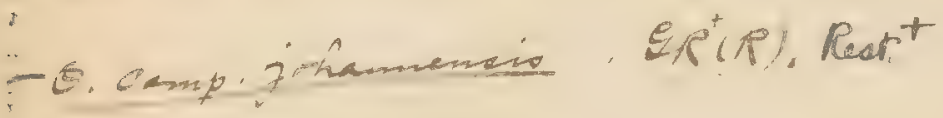

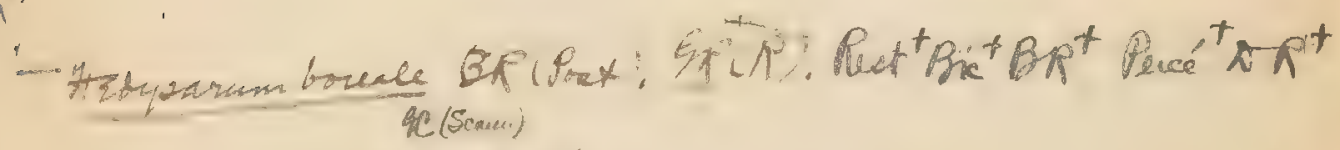

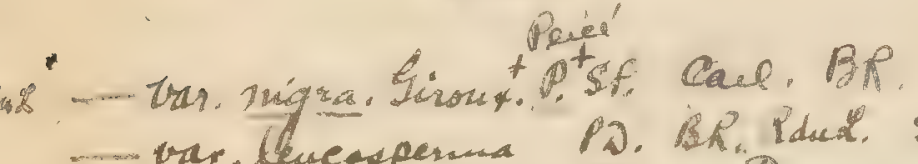
var. Cencespenas P. BR. Pduct. S. cull Bie, Pacé

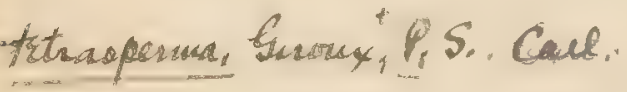

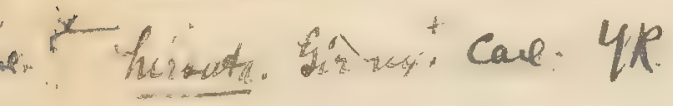


116 ar. alcutica? ? loul

P. amerieana. Q

P. Ariom, ste

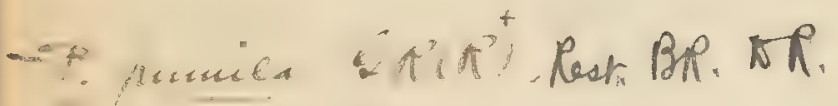


Lathyrus, L. Everlasting Pea.

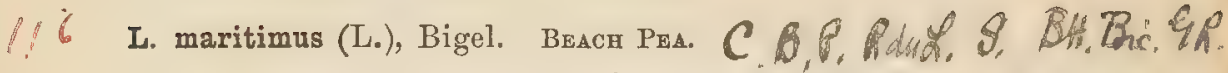

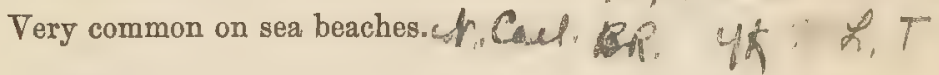

$1 / 7$ L. palustris, L. Margh Pea.

Moist places near the sea; frequent. Aunt Mollys Beach; $C$ BulCost Southwest Harbor; Sea Wall; Great Cranberry Isle (Rand); - Pdec $\mathscr{L}$. Bar Island, Bar Harbor (F. M. Day); - Little Cranberry Islegice. Bà. Seal Harbor (Redfield); - Norwood Coge (M. L. Fernald). if Caul BRt L. Pratensis, L. Firid Pra.

Well established in field, Bar Harbor (Mary Minot). Natu- Sraq. ralized from Europe.

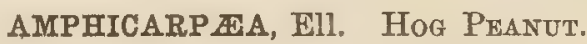

$1 / 9$ A. monoica (L.), Ell.

Damp thickets on Somes Stream (Rand). Apparently indigenous, but perhaps introduced.

ROSACE R. Rose FamiLY.

Prunus, L. Plum. Cherry.

120 P. Pennsylvanica, L. f. WrLo Red Cherrx. C B.P. Pdes Q Q.S.

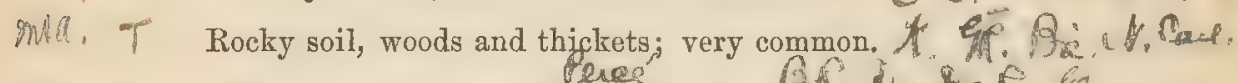

$/ 2 /$ P. Virginiana, L. Cноке Cherry.

$B A, d, \operatorname{baf}$, fic.

Waysides and thickets; frequent. Salisbury Cove, etc. C. A C (Rand); - Somesville (R. \& R.); - Squid Cove (Redfield); - S. Ã.

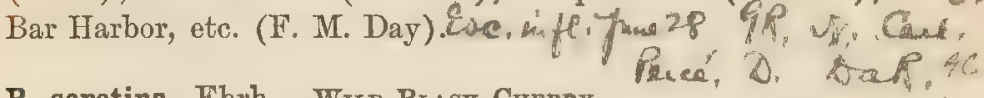

P. serotina, Ehrh. Wird Braok Chrвrq.

Not uncommon about Somesville (Henry C. Jones, and sighinfeyfury others); - Bar Harbor (W. H. Manning). Blooming later than the preceding, which it much resembles. 


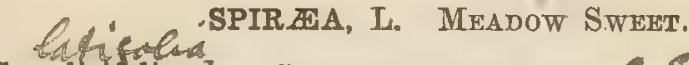

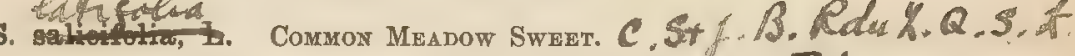
Low grounds and damp hillsides; common. Bie Tad

S. tomentosa, L. Hardhack.

Low grounds; common. C., frome Trus twrothr.

RUBUS, L. RASPBERry. BlackBerRy.

R. odoratus, L, Porple Flowering Ragpherry.

Occasional by roadsides. Emery District; Southwest Harbor (Rand); - Hulls Cove (R. H. Day). An evident escape from cultivation. Adventive from beyond our limits.

R. Chamæmorus, L. Baked Apple Berry. Watace.

Rare. The Heath, Great Cranberry Isle (R. \& R.). Said to C.PD. grow in great abundance near Prospect Harbor, Gouldsborough,

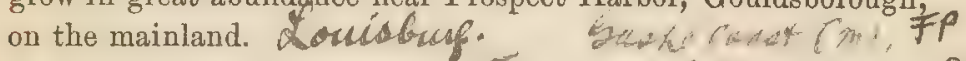

R triflorus, Richards. Woon Ratpeerre Pené gee $P+$

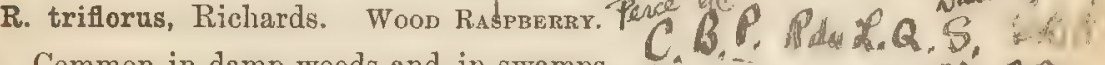
Common in damp woods and in swamps, Raer. Bic. As. Carl, XC. RR.

R. strigosus, Mx. Whd Red Raspberry f Daf hit?

Very common everywhere, especially in clearings and old C. B.At.FP.

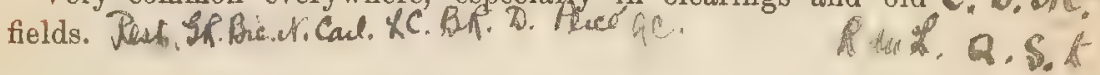

R. villosus, Ait. Hrgh Blackberrr.

Waysides, fields, and thickets; very common. $\mathbf{Q}$

Var. frondosus (Bigel.), Torr.

Frequent. Northwest Cove; about Somesville and elsewhere (E. Faxon, R. \& R.) ; - Bar Harbor (W. H. Manning).

Var. Randii, Bailey.

Low and diffuse, $1^{\circ}-2 \frac{1}{2}^{\circ}$ high, the canes bearing very few and weak prickles, or often entirely unarmed, very slender and soft, sometimes appearing as if nearly. herbaceous; leaves very thin and nearly or quite smooth beneath and on the petioles, the teeth rather coarse and unequal; cluster stout, with one or two simple leaves in its base, not villons, and very slightly if at all 
-S_RduL. Ret ther.

S- pint top(filipenonda) $Q$

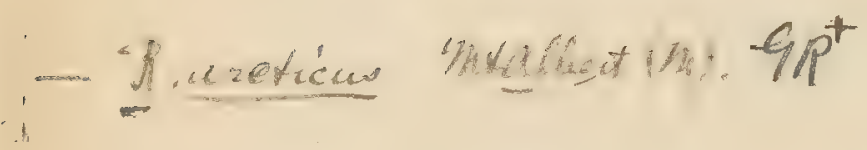

- P. Idacus Pre ${ }^{+}$

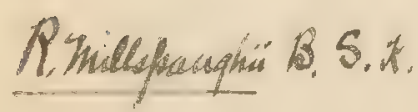

R. vrib of thaime * $8+7$.

R, miquicans, is sig. 
acchemila stal.

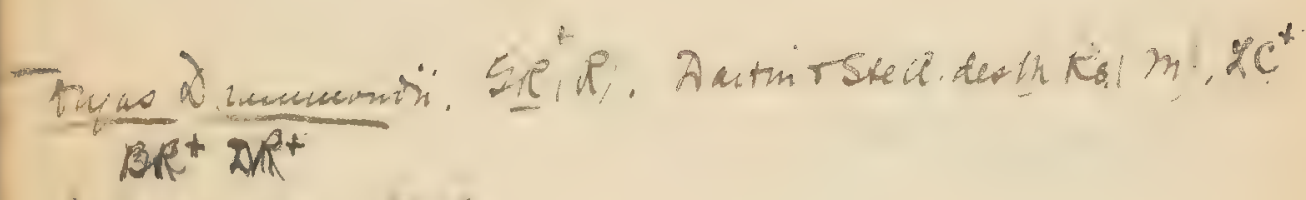

S. virginianum * DaRt:

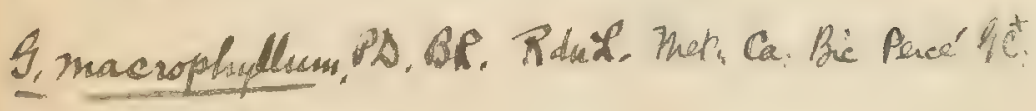

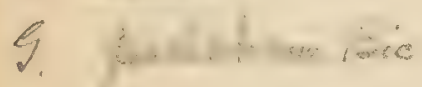

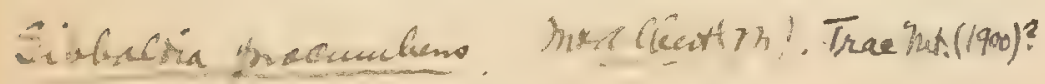


pubescent; flowers half or less the size of those of $R$. villosus; fruit small, dry, and "seedy." Woods, Southwest Valley road (Rand).

2 R. Canadensis, L. Low Blackberry. Dewberry.

Dry fields and roadsides; frequent.

\section{a.}

R. hispidus, L. Running Swamp Buackberry.

Low grounds and by waysides; common. C.

R. setosus, Bigel. Stouter than $R$. hispidus, larger leaved, suberect or ascend- $\mathbf{C}$ \&
ing, the older wood most densely clothed with slender, stiff, slightly reflexed bristles; not evergreen; flowers usually small; fruit reddish black, about $3^{\prime \prime}$ high; leaflets mostly acute, or short acuminate, generally 5 on the leaves of the sterile shoots, and 3 on the flowering branches, short petiolulate or sessile; pedicels and petioles often with a few weak bristles, pubescent. (See N. I, Britton in Bull. Torr. Bot. Club, xx. 278, whence the above description is mainly taken.) Not uncommon. Somesville; Beech Cliff; Oak Hill (Rand).

\section{$/ 35$ D. repens, L.}

\section{DALIBARDA, L.}

Woods; common. Fertile flowers mainly, if not entirely, cleistogamous, appearing rather earlier than the more showy flowers. (See T. Meehan, Proceedings Acad. Nat. Sciences of Phila., 1892, p. 371.)

G. album, Gmelin.

GEUM, L. AVENS.

Thickets; rare. Wasgatt Cove (Wm. H. Dunbar);-Somes-Q. ville (Rand); - near Somesville (Arnold Greene).

G. strictum, Ait. $2 h \times$

Rare. Somesville (Rand). Perhaps introduced. MC, DR, Rdu X, Q G. rivale, L. WATER Avens. Bue. Wi, Call. Thac Juat(15od) BR.

Common in wet fields and meadows in the north and west of C.M.C.S. the Island. Also Long Pond meadows (Redfield); - meadow at $\operatorname{uR}^{2} \mathrm{BiC}$ Schooner Head (Robert B. Worthington); - Cold Brook (Rand). BR. D Dat FF. GC. 
FRAGARIA, L. STRAWBERRT.

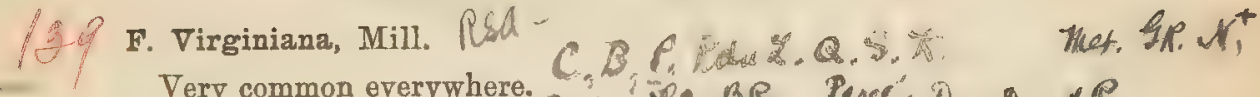
Very common everywhere. Cas SC, BR. Peré, D. AauP.

* F. vesca, $\mathrm{L}$.

Fields and rocky places; rare. Mot, RduL. Bie.M. Cace, Re. BRt.

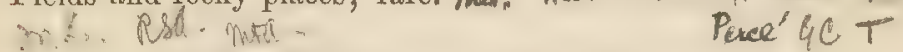

POTENTILLA, L. Cinquefort.

141 P. Norregica, L. RSA $-T$ Pucé fe

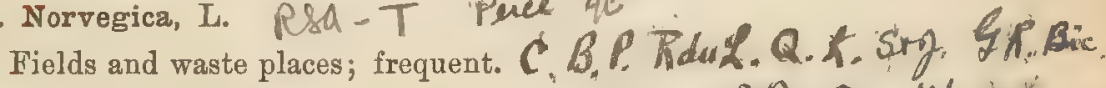

P. Pennsylvanica, I. . i. Carl, BR. D. $4 k$. in

Rare. North of the Island (Wm. C. Lane?).

P. argentea, L. Silvery Cinqueforl.

Dry ground; common.

C. S.x.

P. palustris ( $\mathrm{L}_{*}$ ), Scop. Marsh Potentrila.

Bogs; rare. Somesville (Rand); - Great Cranberry Isle C. B, fie. B. (Redfield). Ca, Bie $\mathrm{AR}^{+}$g]

P. fruticosa, L. Surveby Cinqueforl. 'mela -

Infrequent. Asticou (Wm. C. Lane); - Sargent Mt. (Rand); $\mathbf{B} \mathcal{R}^{+}$. - Jordan Mt. (Arthur Chase); - Southwest Harbor (Annie S.

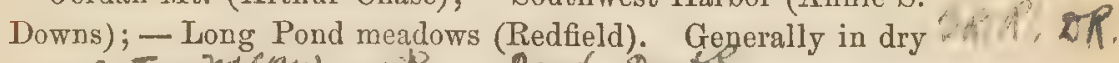
ground. Trachal (Ano) wh Peed Daith.

P. tridentata, Ait. White Potemtrlus sometimespiask at $D$. Tad.

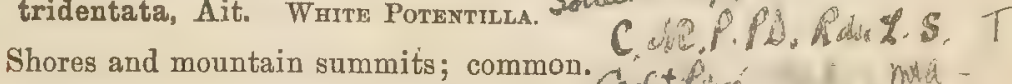

$-\cdots$

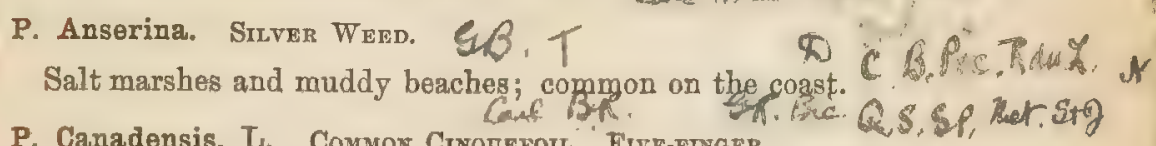

P. Canadensis, I. Common Cineueroll. Fife-fincer。

Fields and waysides; common. The typical form - which is low or dwarf, silky-hairy, with prostrate and decumbent stems is rare. Northeast Harbor (Rand). The common form of the Island is var. simplex (Mx.), T. \& G. This is less hairy and C.Q.* , greener, larger, the ascending stem $1^{\circ}-2^{\circ}$ long, seldom if ever sTy 


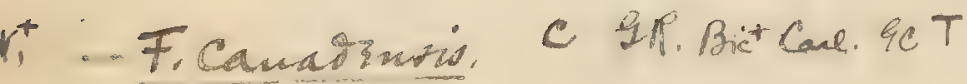

Famericana, Po, IR.

$\exists$. Hayphack, 1 duL.

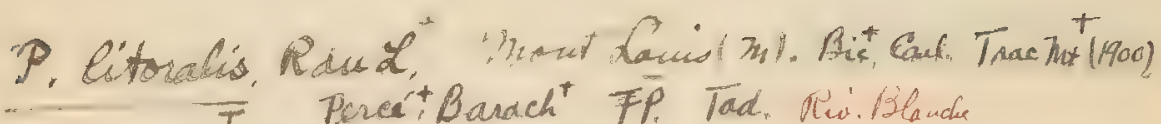

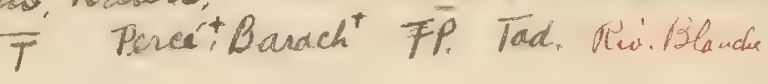

P. argutse. Reat.

P. niver, Biet Pencét

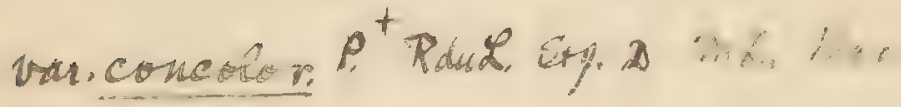




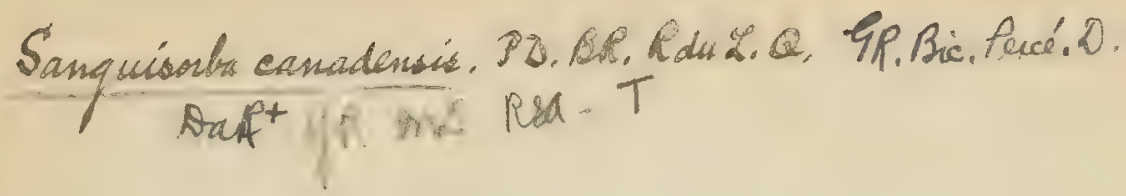

a. igyprepola. 4.

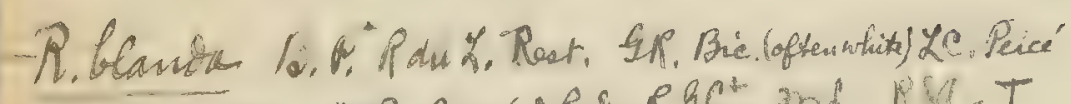

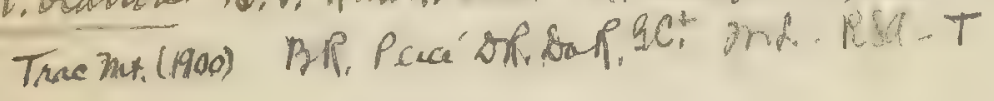
R. astansana. Percet

$$
R \text { - Dact } P^{+}
$$


creeping; from a thicker and harder caudex; leaflets obovateoblong, sometimes almost glabrous. (Torr. \& Gray, Fl. N. A., i. 443; Gray, Man., 5th ed.154.) Although intermediate forms between this and the type are found, it seems that this is a good variety. Observations at Mt. Desert and elsewhere do not accord with those of Dr. N. L. Britton (Bull. Torr. Bot. Club, xviii. 365).

\section{AGRIMONIA, L. AGRIMONY.}

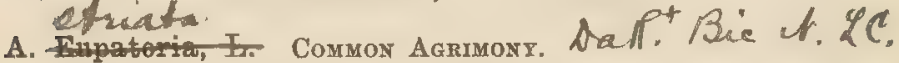

Rare. Woods, Roberts Point, Northeast Harbor; woods, Had- fN, PD. RA,

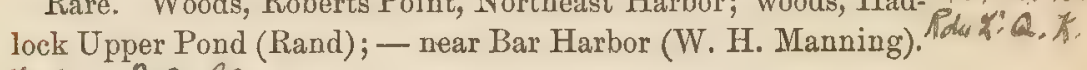
Wher, $B R .9 C$

\section{ROSA, L. Rose.}

$/ 5 /$ R. Carolina, L. SwaMp Rose.

Swamps and borders of streams; frequent.

2 R. lucida, Ehrh. Common Wiln Rose.

Abundant everywhere, usually in dry ground. A form with Cad. Barach downy petioles, in rich soil, north of tong Pond (Redfield). C. B. A C. C

R. humilis, Marsh. T."Ese. ort

A plant answering to the description of this species has been found in woods, Somesville (M. L. Fernald). It is very desirable that the occurrence of this species should be further verified.

R. nitida, Willd. EARLY SWamp Rose.

In bogs throughout the Island, and on Cranberry Isles; $\mathbb{C}$. common.

\section{R. RUbiginosa, I. SweEt Brier.}

Rare. Naturalized from Europe in fields remote from dwell- $P$, $\mathbb{C} .5$ ings, High Head; Bass Harbor (Annie S. Downs); - Seal Har- f. bor (Rand).

/ 56 R. cinnamomea, L. Cinnamon Rose.

Stems $5^{\circ}-8^{\circ}$ high with brownish-red bark, and some straight- $C, C_{2}, Q$ ish prickles; leaves pale, downy beneath; flowers small, pale

$$
\text { ridux. }
$$


pink, cinnamon-scented, mostly double, not showy. Gray, Field, For. \& Gard. Bot., 127. Roadsides, escaped from gardens. Hulls Cove; Oak Hill (Rand). Introduced from Europe.

\section{Pyrus, L. Apple. Pear.}

\section{P. Malus, L. Apple.}

Infrequently spontaneous by waysides, in old fields, etc. $/$. Northeast Harbor; Somesville ; Canada Valley; Sutton Island (Rand).

P. arbutifolia (L.), L. f., var, melanocarpa (Mx.), Hook. Buack Chozeberry.

Common in both wet and dry ground. Very variable in height $6,18$. from $4^{\circ}-5^{\circ}$ in swamps to $6^{\prime}-1^{\circ}$ on mountains. A doubleflowered form, Breakneck Ponds (Rand).

\section{Forma pubescens.}

Pedicels and petioles very tomentose. Not uncommon. Somesville (R. \& R., M. L. Fernald); - Bar Harbor (Mary Minot).

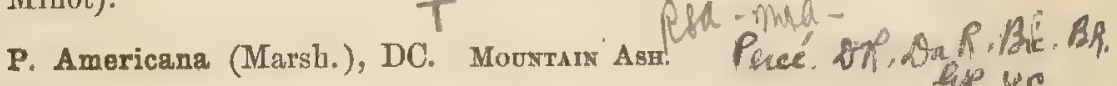
Rocky woods; common. Leaves commonly less taper-pointed, 4 R. XC. and a darker green in color than farther south. Somewhat ap- Micas.

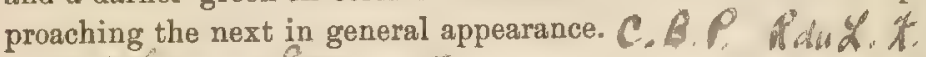

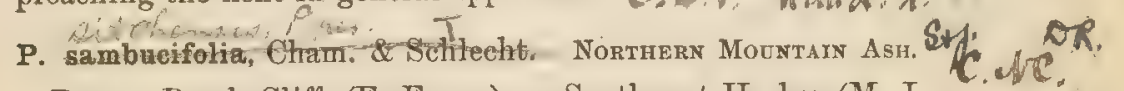

Rare. Beech Cliff (E. Faxon); - Southwest Harbor. (M. L.

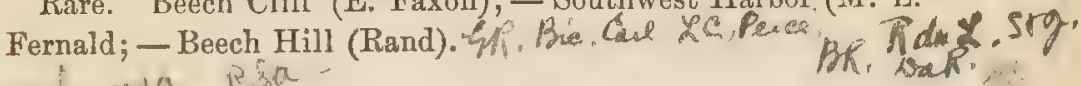
and guta pia

CRAT EgUS, L. HawthorN. ThorN.

C. coccinea, L. Scarlet-rrdited Thorw.

Infrequent. Little Harbor Brook Notch (Rand); - Denning Brook (M. L. Fernald).

Var. macracantha (Lodd.), Dudley.

Frequent by waysides, rocky banks, beaches, etc. The com- 18 , mon Thorn of the Island. 
P. aucuparia, Stg.

b. oryacatha. SP.

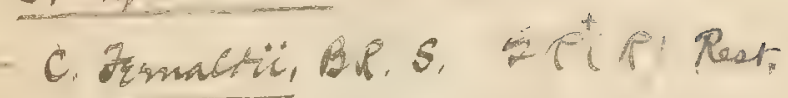

C

!!!!!!!! Q Par

c. 5 .

$\begin{array}{ll}c & k \\ c & k \\ c & k\end{array}$ 


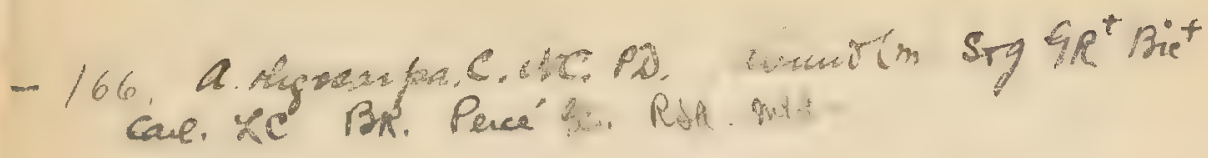

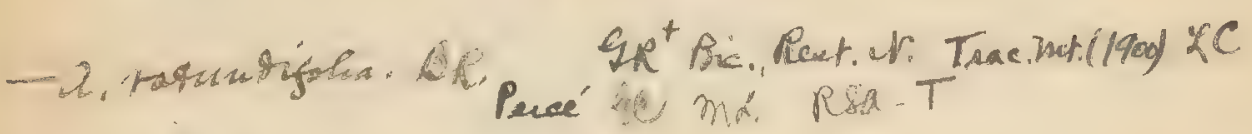

a. Prece

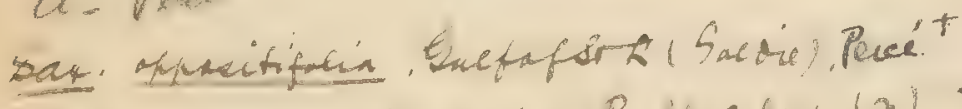

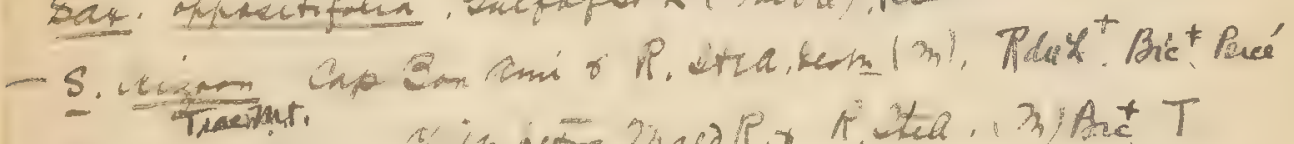

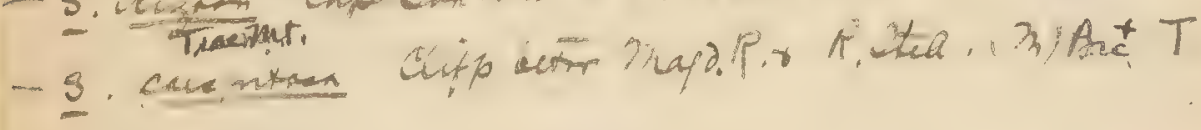

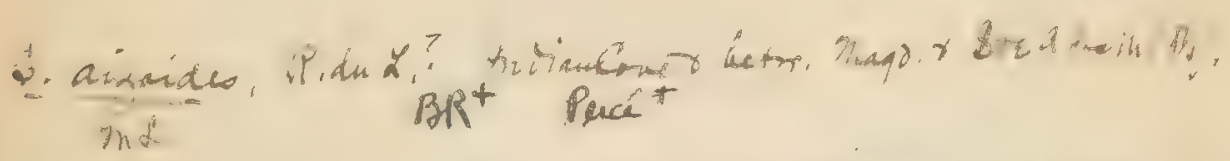

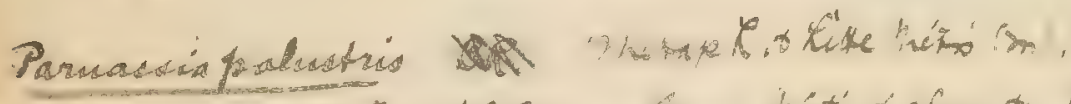

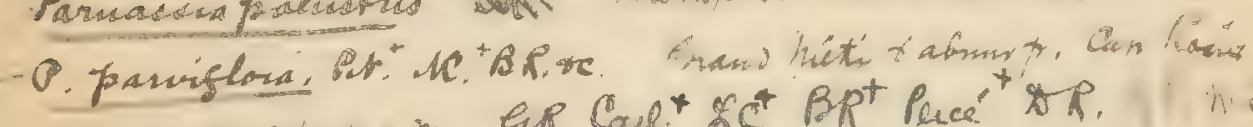

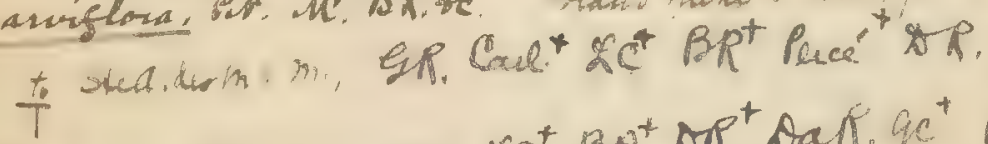

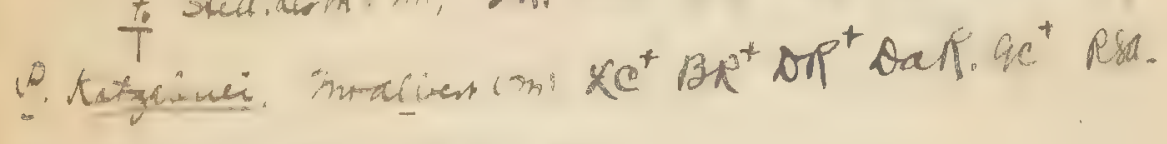

P.caudiniana. Rest. ER. BR $R^{+}$

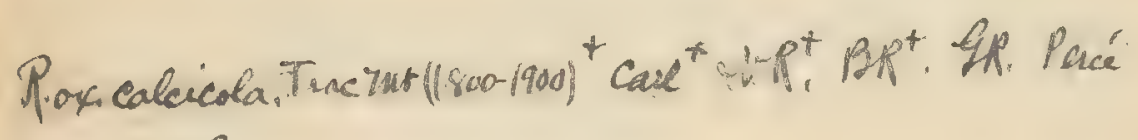
Daf. 
aMelanchier, Medic. Shadbush. Sugar Pear.

A. Canadensis (L.), Medic. Shadresh. 2), AaA. GC. RLA.

Rocky woods; common. Large trees of this species in Little 2,65, Harbor Brook Notch. At. Cal, Bie.

Var. oblongifolia, T. \& G. C MC.S. BR :

Common.

Prece't D

\section{SAXIFRAGACEA. SAXIFRAge Famirt.}

SAXIFRAGA, L. SAXIFRAGE

\section{S. Virginiensis, Mx. Early Saxifrage.}

Rocky places; infrequent. Valley Cove; Dog Mt.; Flying Trowose Mt.; Beech Cliff (Rand); - Sargent Mt. (Greenleaf, Lane \& * Re Rand); - Schooner Head (Clara L. Walley); - ledges on road between Seal Harbor and Hunters Brook (Redfield).

\section{MITELLA, L. MITREWORT.}

\section{M. nuda, L. wha - X. tar. T}

Cool, mossy woods; rare. Hadlock Valley (Redfield); - Cold C P, $\mathbb{S}$ Brook (R.\& R.); - woods, head of Barcelona meadow (Rand). Fdu $\mathcal{L}$.

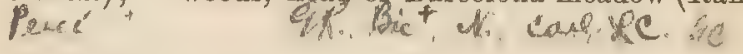

CHRYSOSPLENIUM, I. GOLDEN SAXIFRAGE.

\section{Americanum, Schwein. $Z$ MAt $-T$}

Brooks and wet places; infrequent. On trail between Jordan Pond and Northeast Harbor; Doctors Brook; Little Harbor Brook; Canada Brook; Cold Brook (Rand); - Two Mile Brook (M. I. Fermald). U., \&C. B., \&a. . ".

RIBES, L. Gooseberry. Currant.

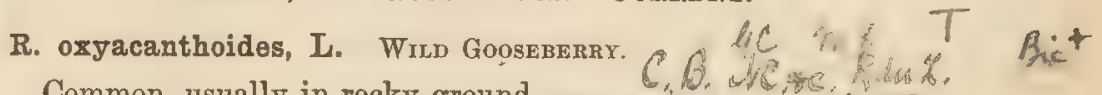
Common, usually in rocky ground. Eve. of Gont. $\mathrm{K}+\mathrm{Tad}$.

R. lacustre, Poir. Peese fre if

Rare. In wet pasture, Great Cranberry Isle (R. \& R.). C R A M

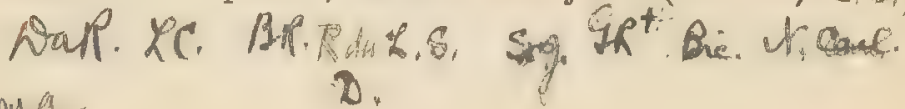


17 R. prostratum, L'Hér. Sxuse Curraxt. BR, Reré, D. DaR, Caul.

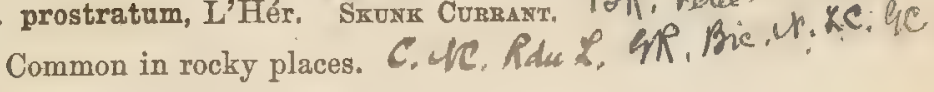

172 R. floridum, L'Hér. Wild Brado Currant.

Uncommon. Clearing, Canada Valley (Rand); - on Somes Stream (R. \& R., M. L. Fernald); - Beech Hill (Redfield). Without doubt introduced in the last-named station, and doubtless escaped from cultivation in the others.

$/ 73$ R. rubrum, L. Red Curraxt.

Sparingly escaped from cultivation. Beech Hill, etc.; near $\mathscr{G}$. High Head, remote from dwellings (Rand).

\section{CRassulace e. Orpine Family.}

SEDUM, L. Stonecrop. ORpine.

S. ACRE, L. Mossy Stonecrop.

Sparingly escaped from cultivation to roadsides, rocky places, $/$, B , Bic, etc. Roadside, Southwest Harbor (William H. Dunbar); established in abundance on rocks, Southwest Harbor (Henry L. Rand) ; - among stones, near the cemetery, Somesville (Redfield). Naturalized from Europe.

S. Telephium, L. LitVe-For-Ever.

Escaped from cultivation to roadsides and fields; frequent. $\mathrm{Cb}_{2}$. de, Southwest Harbor; Fernald Cove; Great Cranberry Isle Thet, (Rand); - Hulls Cove (F. M. Day); - Somestille (R. \& R.). Adventive from Europe. Radun, $v_{0}, D$,

S. Rhodiola, DC. Roseroot.

Rare. Dog Mt. (Henry C. Jones, Rand);-Egg Rock (Henry C. Smith).

\section{DROSERACE E. Sundew Family.}

DROSERA, L. Sundew.

D. rotundifolia, L. RockD-LEated SUNDEW.

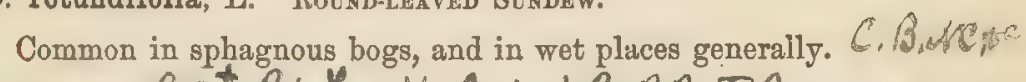

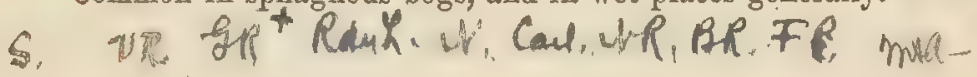




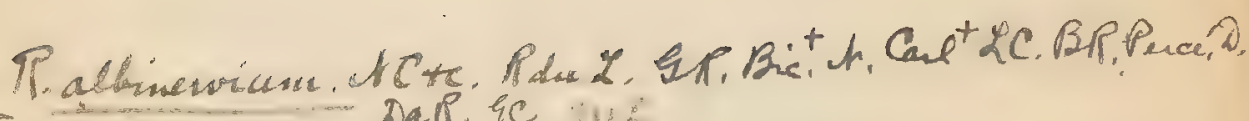
inta- $T$

Drot.comoer SR

X. Congufolin. Ipt

D.eineavis IRt 


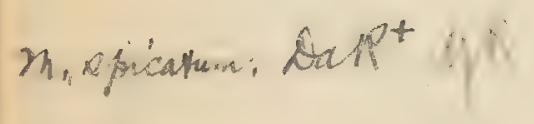

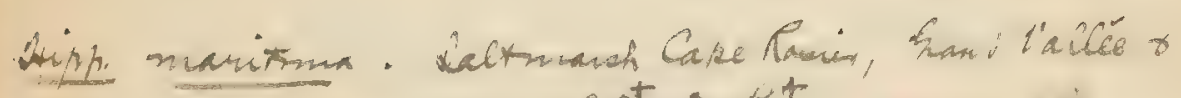

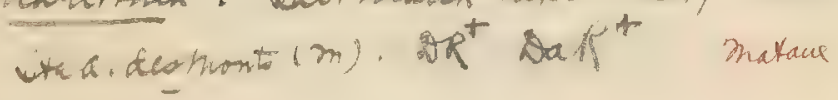


D. intermedia, Drev. \& Hayne, var. Americana (Willd.), DC.C. S S S g, Bogs and borders of ponds; common.

HAMAMELIDE 2 . WitoH HaZeL FaMiLY.

hamamelis, L. Witch Hazed.

179 H. Virginiana, I.

Occasional in woods and by roadsides. $*$,

Halorageze. Water Milfoil Family.

MYRIOPHYLLUM, L. WATER MiLfoId.

$/ 80$ M. verticillatum, $L$.

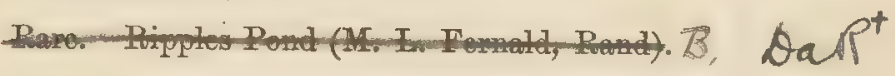

\section{Proserpinaca, L. Mermaid Weed.}

P. palustris, L.

Rare. Meadow at head of Northeast Creek (Rand, M. L. Fernald).

\section{HIPPURIS, L. MARE'S TAIL.}

182 H. vulgaris, L. $n$

Uncommon. Shallow pools at Sea Wall and vicinity, near $\mathbb{B} R^{+} \chi C^{-1}$ op shore (Henry C. Jones, Elizabeth G. Britton, Rand); - marsh, Great Duck Island (Redfield); - not rare, Great Cranberry Isle (Rand, Redfield, Arnold Greene). 17/7

\section{CALLITRICHE, L. WATER STARWORT.}

C. verna, L.

Frequent in muddy streams and ditches. Ditch, Bass Harbor (Rand); - Somes Stream (R. \& R.); - Hunters Brook (Redfield); - Two Mile Brook; Northeast Creek (M. L.

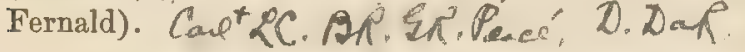




\section{MELASTOMACE E. Melastoma Familx.}

\section{RHEXIA, L. Meadow Beauty.}

\section{R. Virginica, L.}

Sandy or gravelly pond shores; infrequent. Duck Brook meadows; Breakneck Ponds; Great Pond (Rand); - Eagle Lake; Witch Hole (Redfield).

LYTHRACEAE. Loosestrife Family.

DeCOdon, Gmelin. Swamp Loosestrife.

D. verticillatus (L.), Ell. Nescea verticillata (L.), HBK.

Rare. Swamp, Somes Pond (R. \& R.).

\section{onagraCE $\mathbb{E}$. Evening Prinrose Familt.}

LUDWigia, L. False Loosestrife.

L. palustris (I.), Ell. Water Purslane.

Abundant on flats, Ripples Pond (Rand). The only station $S$ m, thus far reported on the Island.

\section{EPILOBIUM, L. WILLOW HERB.}

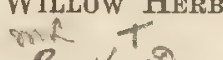

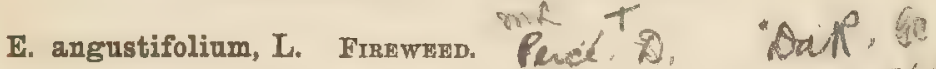

Common, especially in clearings and in burnt ground. C, St, B, R R F'orma albiflorum.

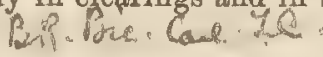

Flowers pure white. Little Cranberry Isle (William H. Dunbar).

/88 E. lineare, Muhl. D.

Bogs and in wet ground; common. A simple form approach- $C, B, M C, S$, ing $E$. palustre, Western Mt. (Rand) A P $^{+}$

Forma latifolium.

Leaves larger, often $4^{\prime \prime}$ broad and $2^{\prime}$ long. Bog, north of Beech Hill (Rand). 


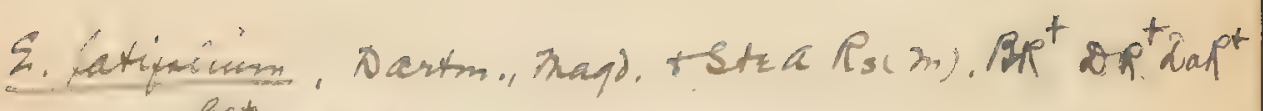
$e^{t}$

- Siliff ge Suta

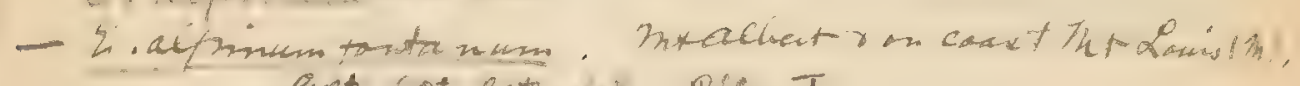

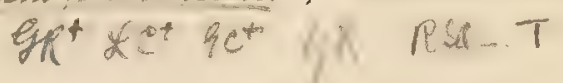

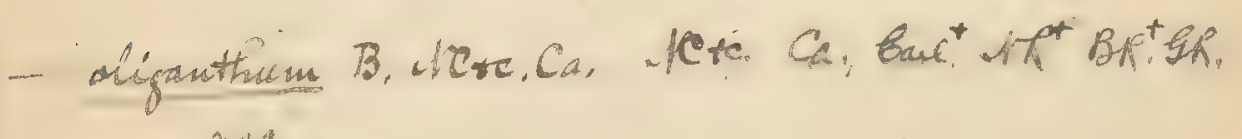
meta.

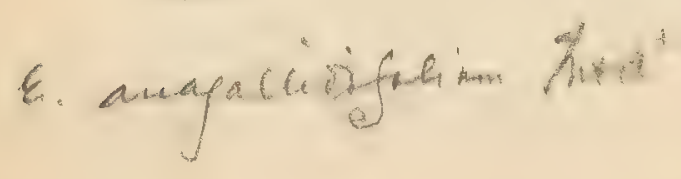




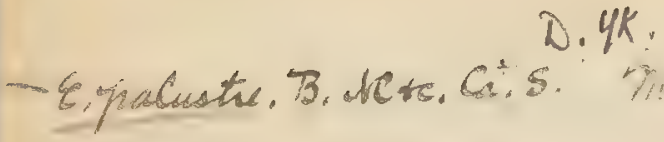

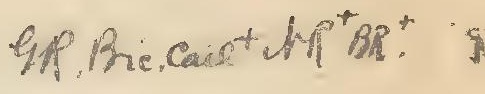

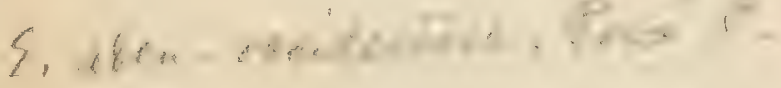

$-\theta+P a r$.

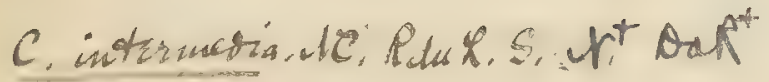

C. Lustriana, Q. * . 
E. strictum, Muhl.

Bogs and wet ground; frequent. Near Sea Wall; High Head; Southwest Harbor; Great Cranberry Isle (Rand); - Salisbury ald . Ast. Cove (Clara L. Walley). A form said by Prof. Trelease to be $\mathrm{L}^{+}$ "perhaps crossed with $E$. lineare," bog, north of Beech Hill (Rand).

E. coloratum, Mubl.

Apparently rare on the Island. Bog, north of Beech Hill; $E_{1}+r^{+}$ Southwest Harbor (Rand). Pl: $^{\mathrm{r}}$

A hybrid, $E$. coloratum $\times E$. adenocaulon, roadside, south of Bubble Pond (Rand).

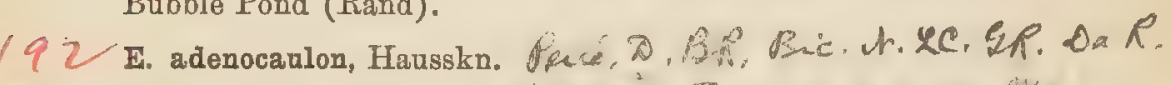

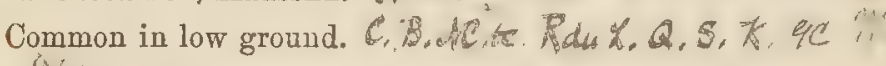
R $A$

\section{cenothera, L. Evening Primrose.}

Pe 2

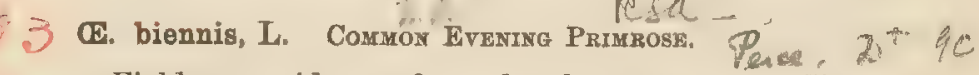

Fields, waysides, and sea beaches; common. Variable. A Sor T.P. Q S

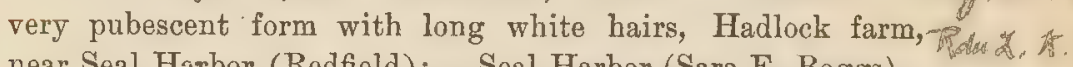
near Seal Harbor (Redfield); - Seal Harbor (Sara Ey Boggs).

CE. pumila, L.

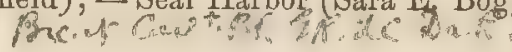

Common in dry șoil. Often much dwarfed. C.B.

CIRCAFA, L. Enchanter's Nrghtshade.

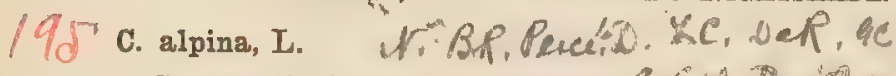

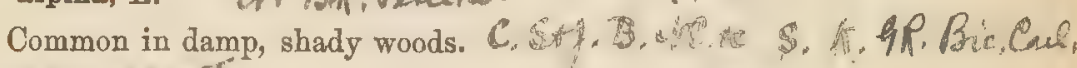
$n:$ nit $\cdots$

\section{CUCURBITACE A. Gourd Famit.}

eCHINOCYSTIS, T. \& G. Wild BaLsam Apple.

E. lobata (Mx.), T. \& G.

Extensively cultivated throughout the Island, and often spon- B. 2,4 . taneous and persistent in waste places and by waysides. Bar Harbor; Southwest Harbor, etc. (R.\& R.) Adventive from the West. 


\section{FICOIDE $A$.}

MOLLUGO, L. INDIAN ChICKWEEd.

M. vertioillata, L. Carpet Weed.

Rare. Bar Harbor (W. H. Manning). A very lately introduced weed on the Island. Probably naturalized from Tropical America.

\section{UMBELLIFER A. Parsley Family.}

daucus, L. Carrot.

D. Carota, L.

Old fields; occasional. Long Pond (Redfield);-Sea Wall (Rand); - Bar Harbor (Mary Minot).

conioselinum, Fisch. Hemlock Parsley.

\section{c. Canadense (Mx.), T. \& G. NAR. MM RS}

Wet woods and meadows; frequent. Seal Harbor; Long Pond C S 1 , P le, meadows (Redfield); - Hadlock Upper Pond; Little Harbor Thto 4.

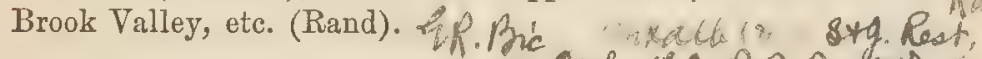

\section{HERACLEUM, L. COW PARSNIP.}

\section{H. lanatum, Mx. Da $R, \&$, Nor. D. mil - T}

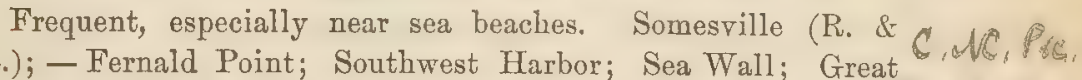
R.); - Fernald Point; Southwest Harbor; Sea Wall; Great
Cranberry Isle, etc. (Rand); - Baker Island (Redfield).

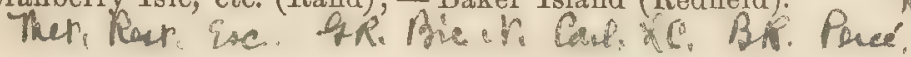

PASTINACA, L. PARsir.

P. sATrVA, L. Mif

Roadsides, waste grounds, and old fields; frequent. Northeast Harbor (Henry C. Jones); - Long Pond; Somesville; Southwest Harbor; Bar Harbor; Canada Valley, etc. (Rand). Naturalized from Europe. Care. 


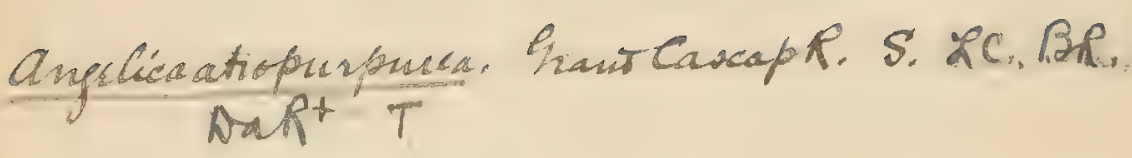

Anthrisous Cerefohion, Matame 


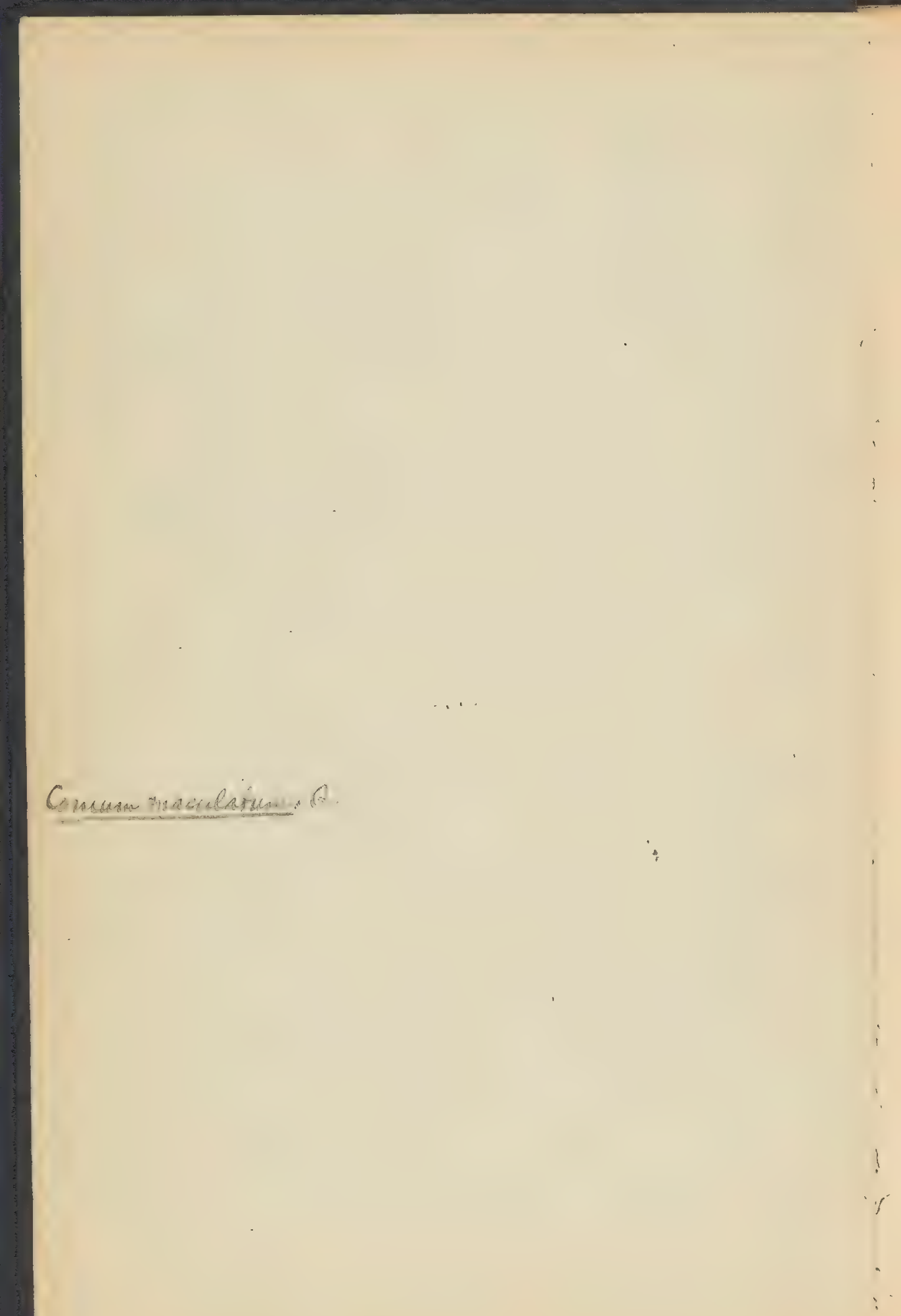




\section{LIGUSTICUM, I. LOVAGE.}

202 L. Scoticum, L. Scoten Lovage. Silk. Aah.

Sea beaches, banks and rocks near salt water; common. C.P. Rdu

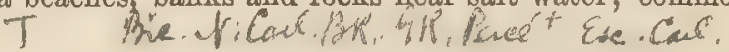

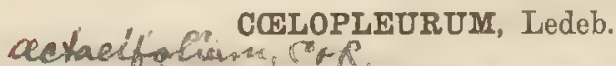

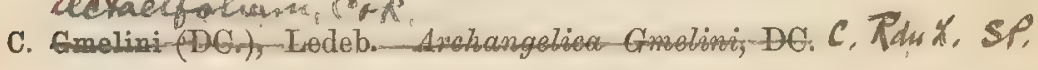

Frequent on the seashore in damp ground. Plant very

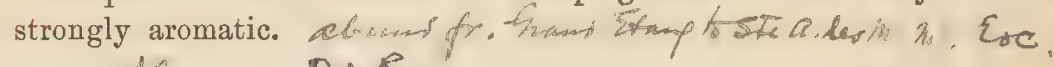
Oet

SIUM, L. Water Parsir.

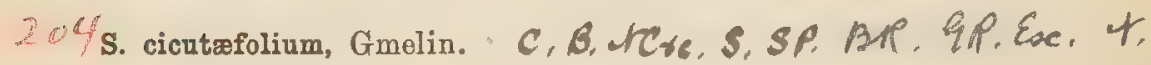
Brooks and pond shores; common.

\section{CARUM, L. Caraway.}

2. 1) C. CARUT, L.

Common in fields and waste places about settlements. Natu- C,S J . $\mathrm{dC}$,

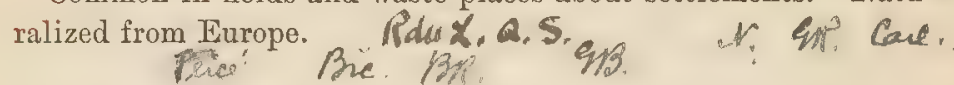

Cicuta, L. Water Hemlock.

2.6 c. maculata, L. D, Dact in

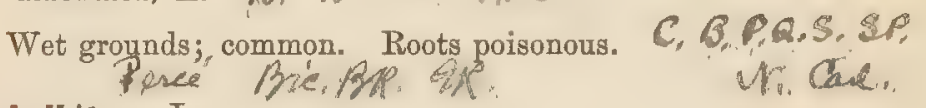

20.7 C. bulbifera, L.

Wet places ; infrequent. Somesville ; Northeast Harbor PD. Car (Rand). Also poisonous. Mh. Aat the

Hydrocotyle, L. Water Pennywort.

II. Americana, L.

Wet places, - woods and meadows; frequent. Hadlock Upper $C_{\text {. }}$ Pond; High Head; Southwest Valley road; Town Hill, etc. (Rand);-Somesville (R. \& R., M. L. Fernald). Stems stoloniferous, especially in late summer. 
SANICULA, L. Black Siakeroot.

S. Marylandica, L. nualous IC Rsa - T.

Wet woods and meadows; infrequent. Little Harbor Brook C, B.PD, Notch (R. \& R., Arnold Greene);-Cold Brook; Long Pond

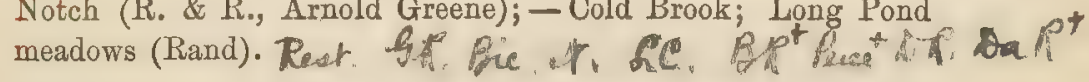

\section{araltace A. Ginseng Family.}

ARALIA, L. WILD SARSAPARILIA.

A. racemosa, L. Spikgnard.

Woods; infrequent. Little Harbor Brook Notch (Rand, Red-Cax, field);-Intervale Brook (F. M. Day);-Hadlock Valley (G. Hunt); - Southwest Valley road; Wild Cat Valley (Rand); roadside east of Seal Harbor; Bubble Pond (Redfield).

A. hispida, Vent. Bristlu Sargaparilitu.

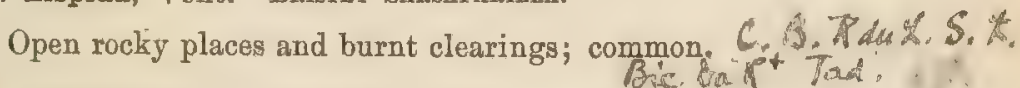

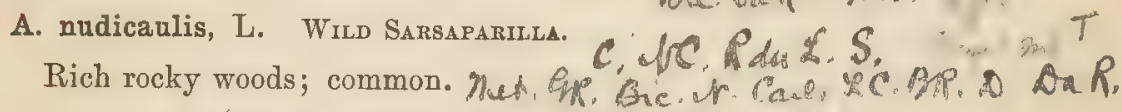
Pins: 40 man.

\section{CORNACEE. Dogwood Famitr.}

CORNUS, L. CorNel. Dogwood,

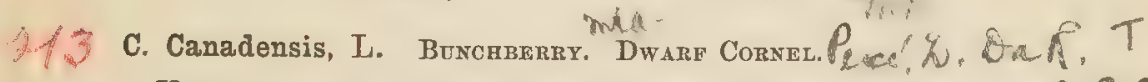

Very common; woods and everywhere. Often blooming until C, G.P. RduLS,

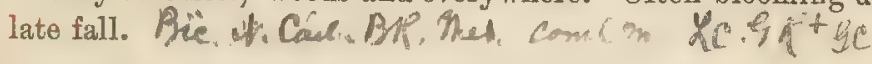

$2 / 4$ C. circinata, L'Hér. Round-leaved Corres.

Woods and thickets; infrequent. Echo Notch (R.\& R.);- B, RAs \&, . . . Little Harbor Brook Notch; High Head; Northwest Cove;

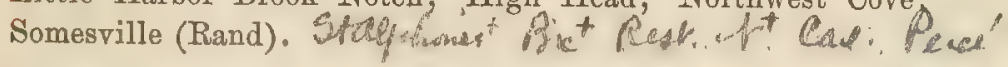

7.15

C. alternifolia, L. f. Alternate-Latyo Cormel.

C.6.5. Pené

Woods and copses; common. "Mabh. RSA. 


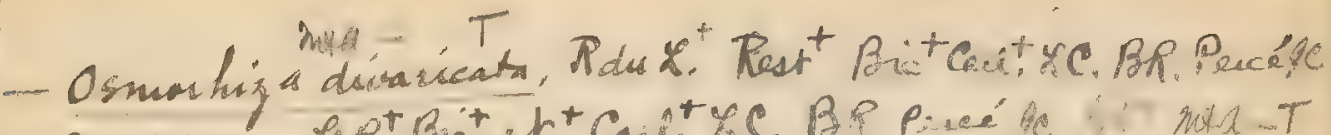

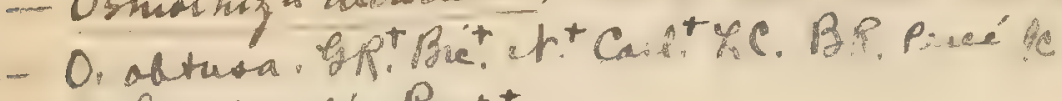
nus $-T$

- O. Conpietulis. Rest.

O. Brevistufis tt

- conciea Pduxi. Ca.

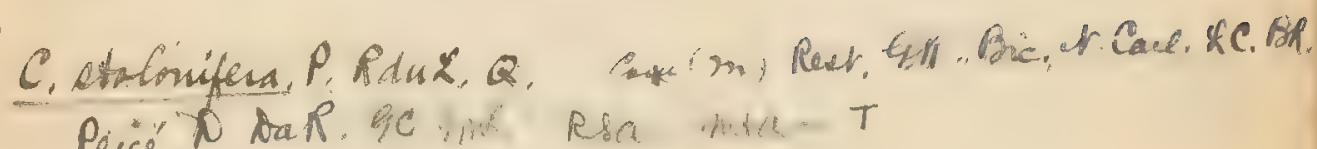
Pricé, to tar. ge the Ra whe- $T$ 
DaR. Rla nuta

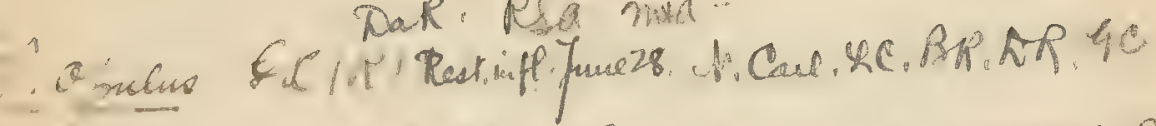

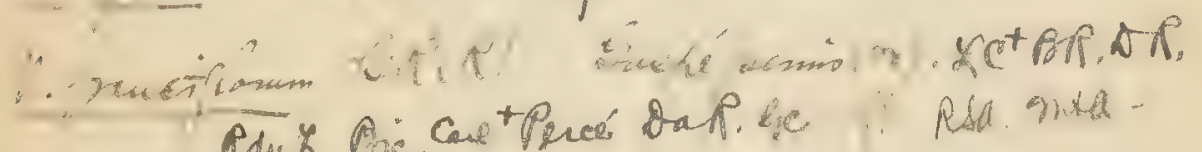

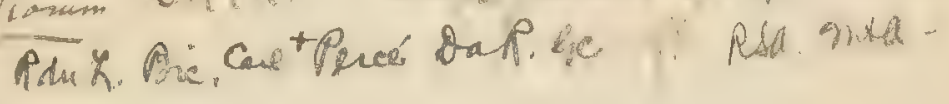

Sym. raemooum! Bie*. 5ymphricaspre racenmasts, Q, 5, K, Tad. 


\section{Drvision II. GAMOPETALE.}

\section{CAPRIFOLIACE EE. Honersuckle Famity.}

SAMBUCUS, L. ELDER.

\section{S. Canadensis, L. Common Elder.}

Frequent in rich soil, but nowhere very abundant. Somes-C.P.P. ville; Northeast Harbor; Southwest Harbor; Gilmore Meadow, etc. (Rand); - Long Pond meadows, etc. (Redfield); - Bar Q Harbor (F. M. Day).

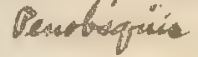

S. racemosa, L. S. pubens, Mx. Red-Berried Elder.

Rocky places and waysides; more common than the last. ${ }_{A}$ C.P.PA. form with yellowish colored fruit, near Northeast Harbor $\mathbb{R} d u \%$. 5 , (Theodore G. White) P A R

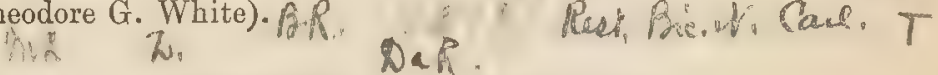
VIBURNUM, L* Arrow WoOd.

If V. lantanoides, Mx. Новвце Веsн.

Common; rocky woods, - especially on mountain brooks, - $C$, and sometimes in low ground.

V. acerifolium, L. Maple-Leaved Viburaum.

Thickets and borders of woods; frequent; rare in the southeastern part of the Island.

V. dentatum, L. ARrow Wood.

Rare. Meadow at head of Northeast Creek (Rand, Redfield, M. L. Fernald).

V. cassinoides, L. WITHE-Rop.

FP. Ti. Hande

Rocky woods, moist banks, and wet places; common. Also on $C$. a the mountain summits. Con. Theintika), S.

LINN AEA, Gronov. TwiN Flower.

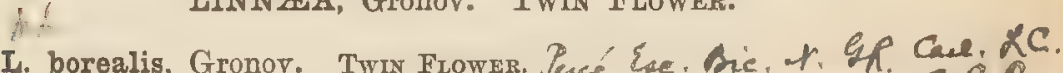

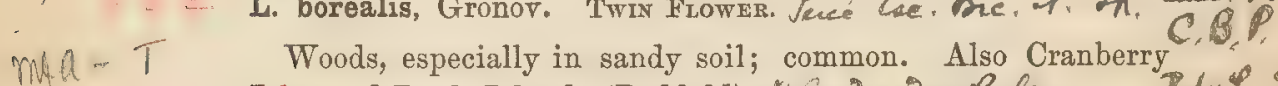

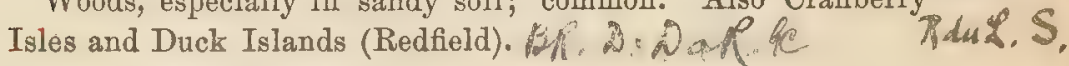




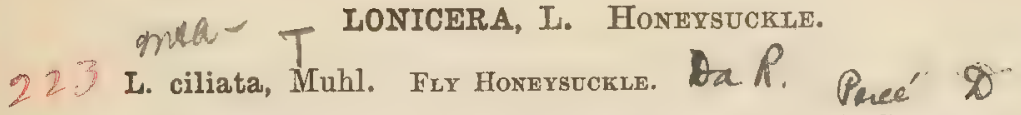

Rocky woods; infrequent. Hadlock Upper Pond; Sargent Mt. C. MC, S, Górge; Little Harbor Brook Notch; Northwest Arm woods, etc. $C_{R^{\dagger}}$ Ric at. (Rand); - Squid Cove (Wm. C. Lane); - Hadlock Valley; Cue. KC. Jordan Pond road; Bear Island (Redfield); - Norway Drive (Mary Minot); - Bald Porcupine Island (W. H. Manning). BR. je

L. cærulea, L. Mountain Fuy Honexsuckle.

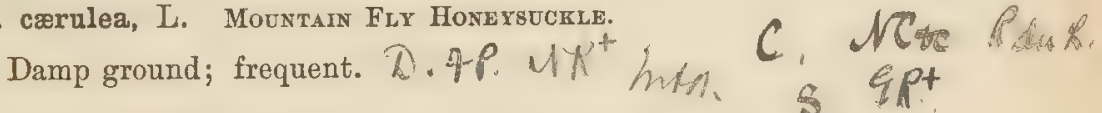
DIERVILLA, Adans. BusH HoNeysuckle.

25 D. trifida, Monch.

Common in rocky ground; woods and clearings. n. it. Gue. KC. BP. Pase' Ba R. Bic

\section{C.MC.RduR.Q.S. QP. $\mathrm{Bic}^{+}$or.}

RUBIACEAE. MADDER FAMILT.

HOUSTONIA, L.

22 H. cærulea, L. Innocents. Butets. Qtaker Ladies. C,

Common in moist grassy places.

mitchella, L. Partridge Berry.

\section{M. repens, L.}

Thickets and woods, especially under Coniferæ; frequent. C, MR.

\section{GAIIUM, L. BeDstraW.}

G. VERum, L. Yellow Bedstraw.

Well established for years in fields, Bar Harbor (Mary Minot, M, L. Fernald), Adventive from Europe.

229 G. Moltugo, L.

Established in field, Bar Harbor (Mary Minot). Adventive from Europe. A somewhat pubescent form with revolute, sharply pointed leaves, Bar Harbor (Mary Minot). 
L. empewirins. $S$

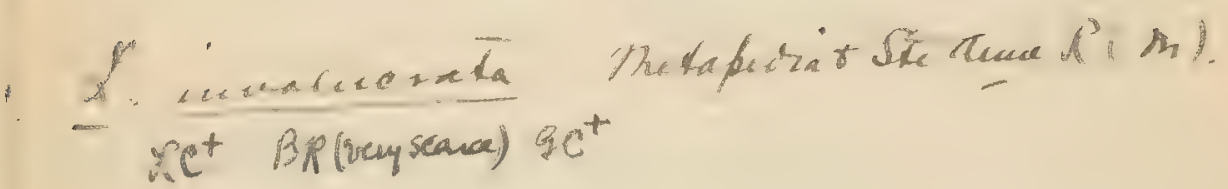

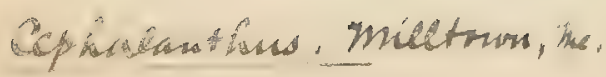


E. erectumittudon Matapioia (RRC)

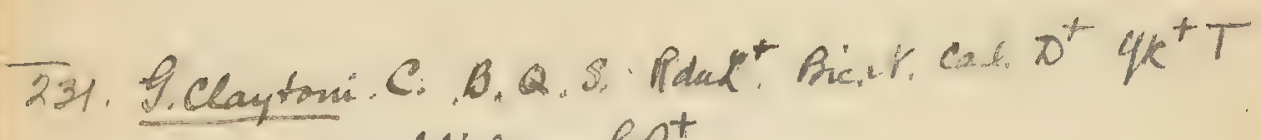
Q. Tificrum subbifformm. ERt

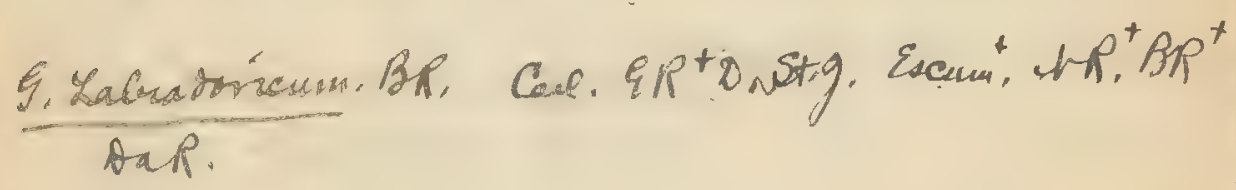

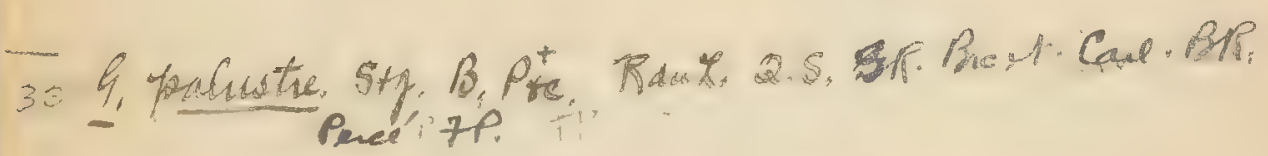

E. Froubdaticuos, matherylm

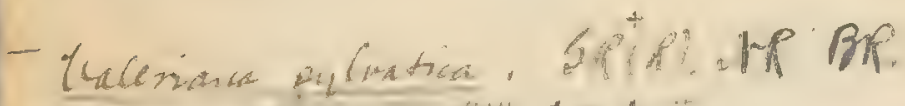

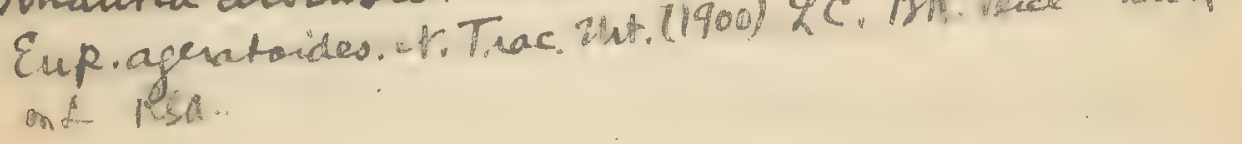


230 G. Aparine, L. Cleangers.

Rare; yet in abundance on beach, Fish Point, Great Cran- Caul berry Isle (Rand). Probably introduced.

23 G. trifidum, L. Smalx Bedstraw.

Common in wet ground. Variable. Ca. Daf.

232 Var. pusillum, Gray.

Not uncommon in cold sphagnum bogs. Little Cranberry C. Isle; Great Duck Island (Redfield); - Southwest Harbor (M. $\mathrm{Ca}^{+}$,

L. Fernald). XC. $A p^{\dagger}$. Apt $p^{+} \mu_{\alpha^{+}}$

G. asprellum, Mx. Rougr Bedstraw. Ar f $4 \mathrm{C} T$

233 RA

Roadsides and low thickets; frequent. Robinson Mt. (Wm. S. . . . , $^{+}$ H. Dunbar); - Somesville; Emery District (R. \& R.); - Sea BR.

Wall; Oak Hill, etc. (Rand). D.

G. triflorum, Mx. Sweet-scented Bedstraw.

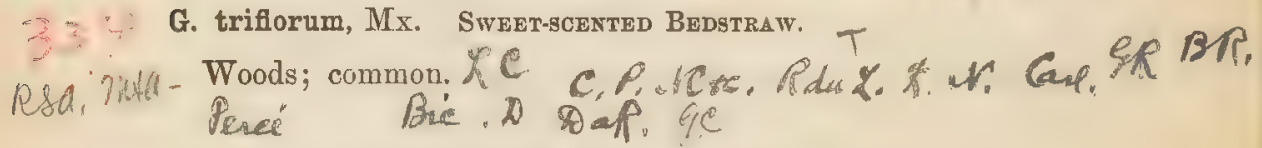

\section{COMPOSIT A. Composite Famity.}

EUPATORIUM, L. ThoroughworT, Da $\mathrm{h}^{+\mathrm{t}} \mathrm{C} T$

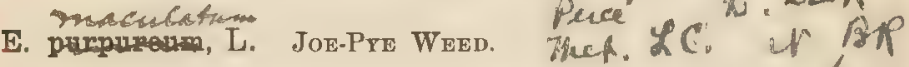

Low grounds; brooksides, and meadows; frequent. A.C., $P$ Q Q,

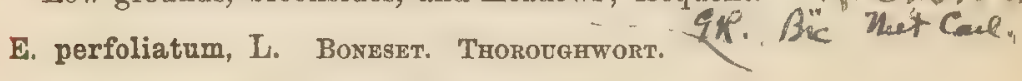

Low grounds and wet roadsides; common.

\section{SOLIDAGO, L. GOLDEN ROD.}

S. squarrosa, Muhl.

Rare; roadsides and thickets. Emery District (Rand, An. T, Q nie S. Downs); - foot of Western Mt. (Rand); - "Salisbury Woods" (Clara L. Walley).

s. latifolia, I. Ge Ral (ones)

Damp, low woods, especially by brooksides; frequent. Head 10,0 of Hadlock Upper Pond; foot of Western Mt.; Little Harbor $/$. Cart. 
Brook Notch; Beech Mt. Notch; and elsewhere (Rand); - path to Bubble Pond; path to Newport Pond; Hadlock Valley (Redfield); - Dog Mt., in dry, open ground (Rand).

\section{S. bicolor, L. WHITE GOLDEN RoD.}

Roadsides and fields; common.

Var. concolor, T. \& G.

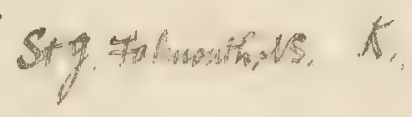

Roadside, south of High Head. The plant, however, is not a very well marked form of this variety.

S. Virgaurea, L.

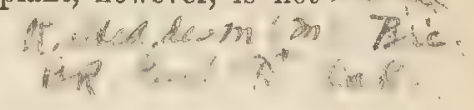

Stem erect, sparingly branched, $4^{\prime}-24^{\prime}$ high, glabrous or pubescent with curled hairs; leaves linear or lanceolate-oblong, $1^{\prime}-4^{\prime}$ long, obscurely toothed, obtuse or acute; heads crowded, 4" long, shortly peduncled, golden yellow; bracts of the involncre linear, acute, glabrous, green, margins scarious; ray flowers 10-12, spreading; disk flowers 10-20; achene pubescent, pappus white. Hooker, Fl. Brit. Isles, 205. (See also Bull. Torr. Bot. Club, xx. 207.) Hio, Southwest Harbor; foot Pemetic Mt.; Great Cranberry Isle; path on Jordan Mt.; Frenchman Camp road (Rand); - Seal Harbor (R. \& R.). This Island form is very like var. angustifolia, Gaud., and var. ericetorum, DC., of the Old World, with lower leaves oblong lanceolate, long petioled, and upper leaves narrower. There are other forms, with narrower, thinner leaves, approaching $S$. humilis, Pursh, but hardly to be placed under that species. Seal Harbor (Redfield); - foot of Western Mt. ; Great Cranberry Isle; Dog Mt. ; east peak of Western Mt. ; Frenchman Camp road (Rand).

\section{Var. Randii, Porter.}

More or less glutinous; stems stout, erect, $1^{\circ}-2^{\circ}$ high, often dark purple, puberulent, or sometimes glabrate below; radical and lower leaves obovate or oblanceolate, acute, serrate, - cauline lanceolate or elliptical-lanceolate, sparingly serrate or entire, glabrous; inflorescence an ample branched panicle or loose virgate thyrse; heads $3^{\prime \prime}$ or more long; outer scales of the involucre mostly ovate or lance-ovate and bluntish, sometimes almost linear and acute, inner ones oblong-linear, yellowish, 
S. Gic, Canata. L, C. $1009 \mathrm{C}^{+}$

ReA-

Rucé

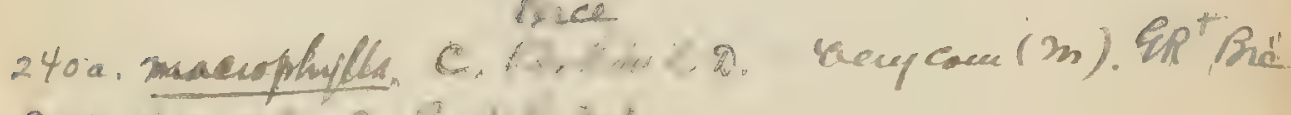

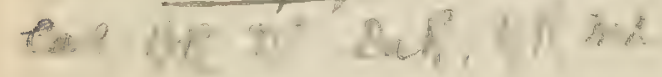

Si chrouditi resa-

8. de........ - $2 x 4 a-$

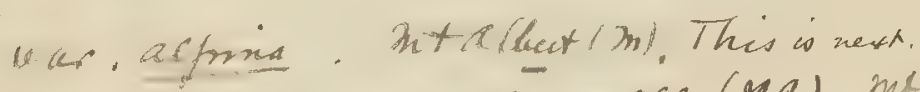

S. multirabiata, ace. (gaa) inta 


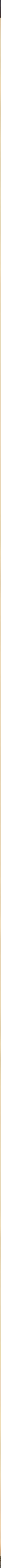


with scarious margins and acute or acuminate tips; achenes pubescent or nearly smooth. Bull. Torr, Bot. Club, xx. 208. Abundant; dry fields, roadsides, and among rocks, especially in the southern part of the Island. Also found on the mountains, distinct, or imperceptibly passing into the next variety. Frenchman Camp; Seal Harbor $(\boldsymbol{R} . \& \mathrm{R}$.); - Sea wall, Long Pond; Northeast Harbor; Southwest Harbor; The Cliffs, Seal Harbor; Hunters Beach Head; Long Pond meadows; Sargent Mt.; Dog Mt.; Pemetic Mt.; Western Mt.; Great Cranberry Isle, etc. (Rand).

Var. monticola, Porter. S. puberula, Nutt., var. monticola, Porter, Bull. Torr. Bot. Club, xix. 129.

Stems $3^{\prime}-12$ high, often slender; inflorescence a short, compact, or sometimes loose thyrse, $2^{\prime}-4^{\prime}$ long; heads $1 \frac{1}{2}{ }^{\prime \prime}-3^{\prime \prime}$ long; scales of the involucre variable, ovate and bluntish or oblong and obtuse, inner ones not elongated. Bull. Torr. Bot. Club, xx. 209. Common on mountain summits, and occrsionally at lower altitudes, even at the sea level. Sargent Mt.; Pemetic Mt.; Jordan Mt.; Western Mt.; Dog Mt.; Hunters Beach Head; Seal Harbor; Great Cranberry Isle (Rand).

Var. Redfieldii, Porter.

Very glutinous; stems stout and rigid, $16^{\prime}-18^{\prime}$ high; leaves thickish or coriaceous; branches of the panicle starting from half-way down the stem or even from the base, strict, erect, bearing short clusters of heads in the upper bracts; heads small, $2^{\prime \prime}-3^{\prime \prime}$ long; scales of the involucre short, more or less scarious. Its inflorescence is strikingly like that of S. juncen, Ait., var. ramosa, Porter \& Britt. Bull. Torr. Bot. Club, xx. 209. Rare. Seal Harbor, etc. (Redfield); - foot of Western Mt.; Great Cranberry Isle (Rand).

S. sempervirens, L. SEa GoLden Rod. $m \mathcal{X}$

Frequent on sea cliffs; common in salt or brackish marshes, $C . .$. and on muddy beaches.

246 S, puberula, Nutt.

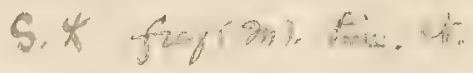

Dry, open ground, and by waysides; common;-less frequent $\vec{R}$. in woods. Very variable in form of inflorescence, etc. A form 
with panicle much-branched, branches erect, much resembling S. juncea, Ait., var. ramosa, Porter \& Britt., in clearings, Sunken Heath, and elsewhere (Rand). A form with inflorescence axillary, much prolonged, Emery District, and elsewhere (Rand).

S. rugosa, Mill. S. altissima, T. \& G., non L.

LC. BR. $D^{+} \operatorname{Dan}^{+}$

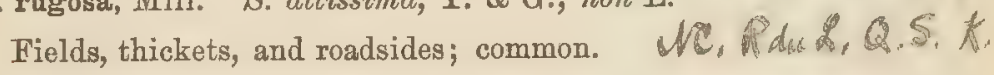

S. neglecta, T. \& G. Swamp Golden Rod.

Swamps, bogs, and meadows. Especially common in sphagnum bogs.

Var. linoides (T. \& G.), Gray.

Sphagnum bogs; infrequent. Great Heath; The Heath, C. Great Cranberry Isle (Rand).

S. juncea, Ait. EARLY GoLDEN Rod.

Dry ground; common. The earliest flowering species of the $\mathbb{R}_{3}, \mathfrak{k}$, genus on the Island. A form more or less pubescent, Hio, Southwest Harbor (Rand). A form approaching var. ramosa, Porter \& Britt., Jordan Mt. (Rand):

S. serotina, Ait.

Rare. Copses, Tung Pond meadows (Redfield); - Salisbury Cove (Clara L. Walley).

Var. gigantea (Ait.), Gray.

Copses and low grounds; frequent. A low form, Long Pond meadows (Redfield). A form approaching S. rupestris, Raf., Long Pond meadows (Redfield).

S. Canadensis, L.

Roadsides, fields, and thickets; common. Puec' $x+B x f, c$

Var, glabrata, Porter.

BR,

Low, slender $2^{\circ}-3^{\circ}$ high, stems glabrous or glabrate below, puberulent above; leaves numerous, crowded, linear-lancoolate, tapering into a long acumination, upper ones entire, lower ones with a few sharp serratures, scabrous on the veins beneath; 
uliginosa B.S. MP BR. ST FP.

S. Almpata Ria- 
Dar iffunctiss. Sty.

var vecubaus, Din, Can."

Ma, ind the de. 
panicles small, with filiform branches; bracts acute or acutish. Roadsides and thickets; infrequent. Near Hadlock Brook, Wasgatt Cove (Rand); - road between Frenchman Camp and Hadlock farm; Frenchman Camp; above Long Pond meadows (Redfield).

\section{5 s. nemoralis, Ait.}

Dry grounds; very common. A form with axillary, much prolonged inflorescence, clusters distant, - between Southwest Harbor and Bass Harbor (Rand).

S. lanceolata, L.

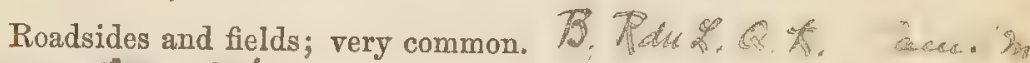
Se. Bre. $2^{t}$

\section{ASTER, L. AsTER.}

\section{A. macrophyllus, L. Great-Leaved Aster.}

Woods and clearings; common. Flowering more abundantly when in clearings or in open ground. Often appearing with few or none of the characteristic root-leaves. To this form, it seems, should be referred specimens collected near Bubble Pond (Redfield), and named by Dr. Asa Gray A. Herveyi.

A. radula, Ait. Rovgh-Leaved Aster.

Low grounds and borders of swamps; common. A form approaching var. strictus, Gray, roadside between Town Hill and Emery District (Rand).

A. undulatus, I.

Rare. Frenchman Camp road (R. \& R.).

A. cordifolius, L. Heart-teaved Aster.

Pdu R -

Wooded banks and waysides; rare. Somesville; Juniper Cove; near head of Northeast Harbor (Rand). Carf.

A. Lindleyanus, T. \& G. Perse'

Dry ground; rare. Southwest Valley road; High Head (Rand); - Thompson Island (Annie S. Downs); - Frenchman

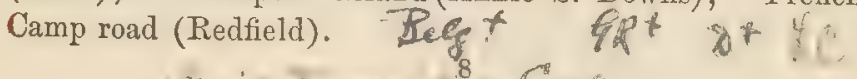

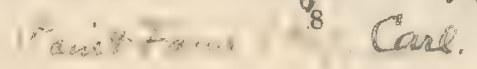




\section{A. polyphyllus, Willd.}

Infrequent and local; in greatest abundance on the mountains. Dog Mt.; Jordan Mt.; Pemetic Mt.; Long Pond meadows (Rand); - Frenchman Camp; on Hunters Brook, near Frenchman Camp (R.\& R.). The forms from Jordan Mt. are much dwarfed.

\section{A. ericoides, $L$.}

Rare. Wayside, road to Jordan Pond (R. \& R.).

\section{A. vimineus, Lam.}

Rare. Frenchman Camp (Rand). Q

A. diffusus, Ait.

Fields, thickets, and waysides; very common. Variable. A form with purple ray flowers and panicles less elongated, Little Harbor (Rand).

Var. thyrsoideus, Gray.

Rare. Seal Harbor (R. \& R.).

\section{A. Tradescanti, L.}

Rare. Southern foot of Western Mt. (Rand).

A. paniculatus, Lam.

Moist ground; frequent and widely distributed, especially in the northem part of the Island. Shore of Northwest Arm, Great Pond; Liscomb Brook, and elsewhere in Emery District; Oak Hill and northward; Somesville; Ripples Pond; Bass Harbor; road to Great Pond, Southwest Harbor, etc. (Rand).

\section{A. salicifolius, Ait.}

Low grounds and roadsides; frequent. Southwest Harbor; southern foot of Western Mt.; Somesville; Beech Hill; Oak Hill; Town Hill; Bass Harbor; Northwest Cove; Eden P. O., etc. (Rand).

\section{A. junceus, Ait.}

Rare. Roadside between Southwest Harbor and Bass Harbor; between Town Hill and Emery District (Rand). 
var, vilicaulis. BP.

$\left.a-\% A^{+} \operatorname{Pace}^{+} A_{1: i}\right)^{+}$ 
A. longifolius, Lam. Not of Gray, Manual, 5th ed.

Low grounds; infrequent. Roadside thicket near Juniper Cove; Cliff walk, Seal Harbor; Meadow Brook, Oak Hill; roadside between Town Hill and Emery District; Eden P. O.; Somesville (Rand).

\section{A. Novi-Belgii, L.}

Abundant everywhere in both wet and dry ground. Very C variable in foliage, size of heads, color of ray flowers, etc. The common form has smooth, thick leaves. A form with very narrow leaves, Somesville; Bass Harbor; Emery Cove; foot of Are. Western Mt.; Pond Heath, etc. (Rand). Forms of this species $\mathrm{Z}^{+}$ apparently pass into A. puniceus, L., var. lucidulus, Gray.

Forma albiflorus.

Ray flowers pure white. Cliffs east of Seal Harbor (Rand). Forma roseus.

A salt marsh form, low, only $1^{\circ}$ high; stems dark purple; leaves linear, thick, with purple midrib, the lower stem leaves bearing abundant axillary clusters of small leaves; rays bright pink. Near Bass Harbor (Rand).

Var. litoreus, Gray.

Salt marshes or wet shores; infrequent or rare. Month of Northeast Creek; Somes Harbor; Great Cranberry Isle (Rand). Forms approaching this variety are abundant, but the variety itself is seldom found.

A. patulus, Lam.

Rare. Mendow, head of Northeast Creek (M. L. Fernald); Town Hill road, Somesville (Rand).

$275^{-A}$ A. tardiflorus, $\mathrm{L}$.

Rare. Wood road to Broad Cove; road from Town Hill to Thomas Bay (Rand).

A. puniceus, L.

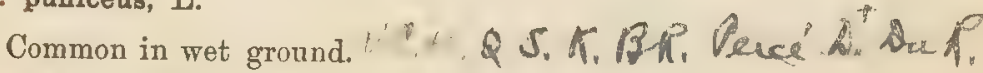
esa $T$ 
Var. Iucidulus, Gray.

Frequent in wet places and moist ground. Seal Harbor (Redfield); - Town Hill; Emery Cove; Youngs District; Eden P. O.; etc. (Rand). Apparently more abundant in the northern part of the Island.

Var. lævicaulis, Gray.

Infrequent. Beech Mt. Notch (Rand); - stream south of $\bar{D}^{+} \mathrm{Da} \boldsymbol{N}^{+}$ Bubble Pond (R.\& R.).

A. umbellatus, Mill. Diplopappus umbellatus, T. \& G. RS1/4rin)

Roadsides, fields, wood clearings, and dry places; very $P$ fre, 8

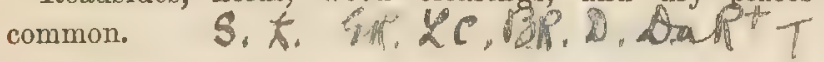

A. acuminatus, $\mathrm{Mx}$. GC RS nut T

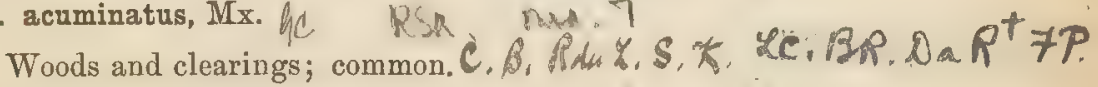

A. nemoralis, Ait.

Peat bogs and open swamps; common. Also summit of Green Mt. in boggy depressions (Rand.) S,

Var. Blakei, Porter.

Stems $1^{\circ}-21^{\circ}$ high, simple, or often branched, inclined to be flexuous; leaves not crowded as in the type, $2 \frac{1}{2}^{\prime}-3^{\prime}$ long, $\frac{1}{2}-\frac{3}{4}$ 'broad, lanceolate, coarsely toothed or entire, margins not revolute, thinnish; heads few or several, sometimes solitary, showy; rays lilac-purple. - Intermediate between $A$. nemoralis and $A$. acuminatus, to both of which some of its forms make a near approach. North border of Somes Pond (Rand).

\section{ERIGERON, L. Fleabane.}

E. Canadensis, L. Horsewed. Butrerweed.

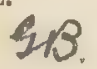

A common weed by roadsides and in waste places, becoming 2 of C. yearly more abundant everywhere on the Island. $G_{0}$ of Ri. Cal.

E. strigosus, Muhl. Smaller Daist Fleabane.

Fields and waysides; common. Rays sometimes more or less deeply tinged with violet. $0^{*}$ S.Mr. 413. 
A. angustus, $C^{*}$

a. oubulatus $\mathrm{B}^{+}$

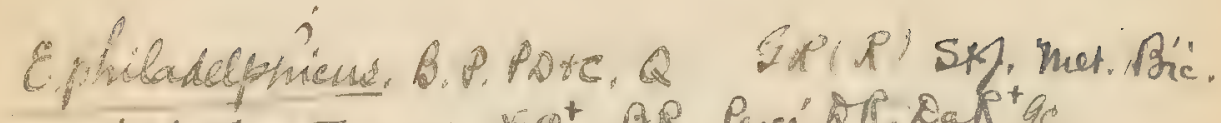

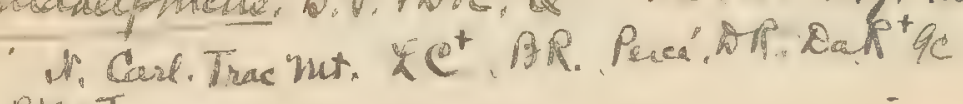

-. Ras $T$

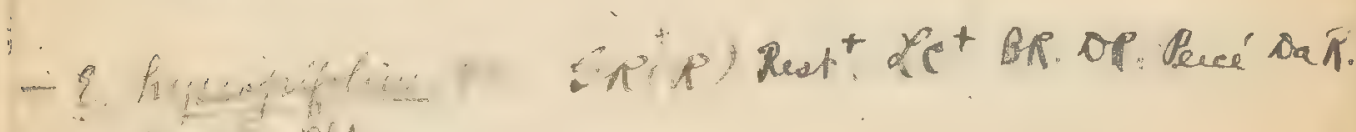

- ite pla

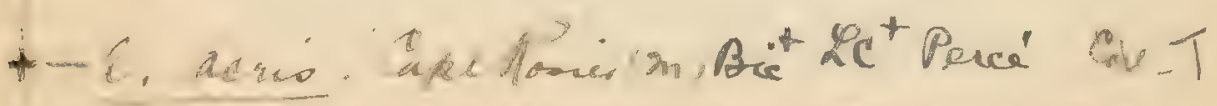

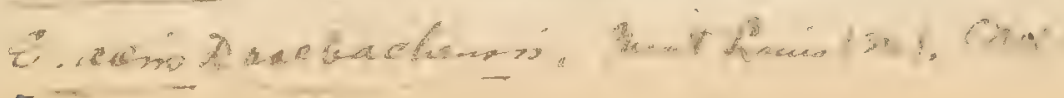




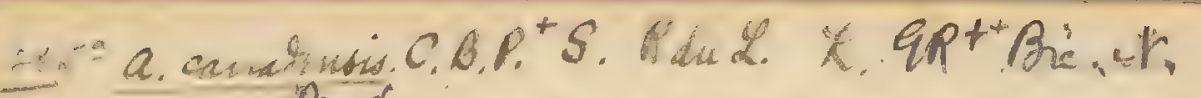
Prece

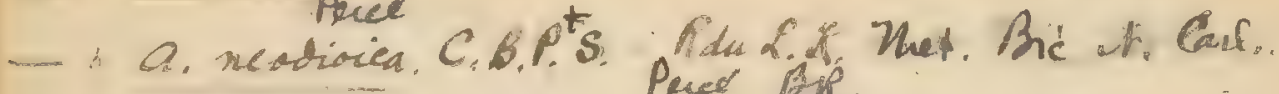

a. ngelecta. $\mathcal{K}$

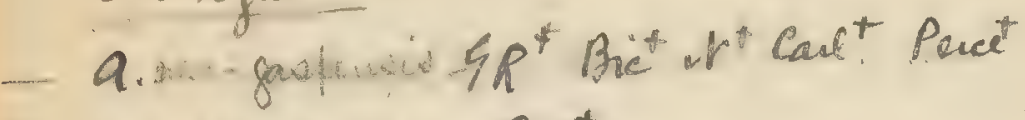

- a. kacteride Qppt

Cunaph. accidentalis. EP(R) Get Pivé, LC Int Rsa

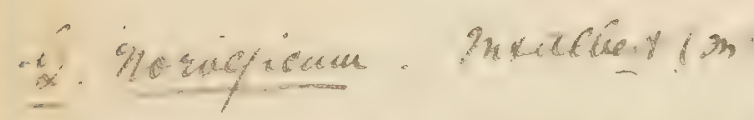

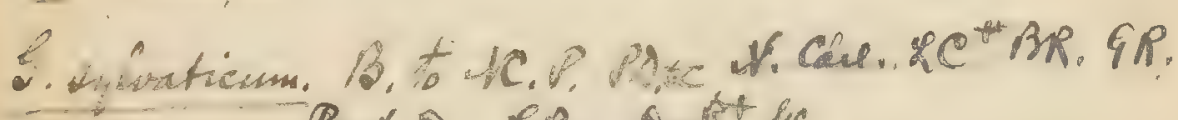
Pacé, D. Pis. oat he

Bunín tréminm Q. X. Can, MP.

$210^{4 i}$

a. tifize Q 
ANTENNARIA, Gærtn. EverLASTING.

A. plantaginifolia (L. ), Hook. Mouse-ears, Iadies' Tobacco.

Dry soil; common.

anaphalis, DC. Pearly Everiastigg.

A. margaritacea (L.), Benth. \& Hook. Añtennaria margaritacea (L.), R. Br. Pearlit everlasting. 9P. Brenth Cand,

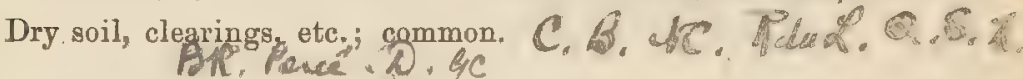
GNAPHALIUM, L. CUDWEED.

G. polycephalum, Mx. Swhet Everlastivg.

Dry fields; common. Very sweet-scented. Ca. T, MP.

G. decurrens, Ives.

Fields, in sandy soil; infrequent. Sawyer Cove; Southwest Harbor; Seal Harbor (Rand); - Little Cranberry Isle (Redfield).

G. uliginosum, L. Low Cudwed. D. Peuce'

Roadsides; common in damp soil. Also Green Mt. (Redfield).

$$
\text { ambrosia, L. RAGWEE. BR, } 7 . \text { Care, }
$$

A. artemisiæfolia, L. RAGWEED.

A common weed in waste places. Many people suppose its pollen an effective cause of hay fover, and find relief on the Island owing to the supposed absence of this plant. This relief however, it seems, must be attributed to some other cause, as the plant in question grows everywhere, - even on the Cranberry Isles, - in more or less abundance, and is spreading with the increasing settlement of the Island.

\section{RUDBECKIA, L. CONE Flower,}

R. hirta, L. Yellow DaIsY. Black-Eyed Sugay.

Naturalized in grass felds from the West. Very common 4 . about Somesville and Bar Harbor, and more or less abundant all over the Island. A fastigiate form, Seal Harbor (Redfield).

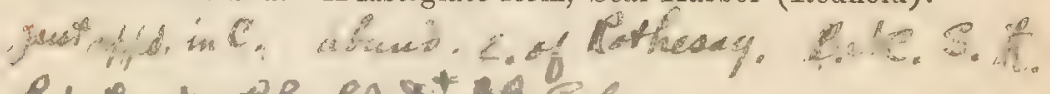

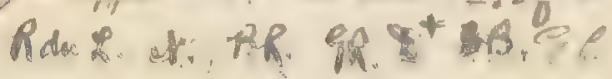


HELIANTHUS, L. SUNFLOWER.

H. annuus, L. Common Sunflower.

Waste places, Fernald Point; Bar Harbor (Rand); - Seal Harbor (Redfield). Escaped from cultivation.

BIDENS, L. Bur-Marigold.

B. frondosa, L. Beggar Troks. Devil's Prtchfork.

Low grounds, wet places, and damp roadsides; common, and spreading. the gr.

B. cernua, L. Smaller Bur-Marjgold.

Wet places; frequent. Sawyer Cove; Mill Cove; Norwood Cove; Valley Cove; northern foot of Beech Hill; Southwest Harbor, etc. (Rand); - Somesville (R. \& R.); - Great Cran- F P berry Isle (Redfield).

\section{ANTHEMIS, I. Chamomile.}

A. Cotula, L. Mamuta Cotula (L.), DC: Maxweed.

Common by roadsides and in waste places. Naturalized from Europe. S, Y $\%$, $4 k+$

\section{ACHILLEA, L. YARROW.}

A. Millefolium, L. Common YarRow. $y k+i c$ n. $T$

Dry soil; common in settlements, and often remote fromC. dwellings. Naturalized from Europe, but also indigonous. a. $3,1-9 R$

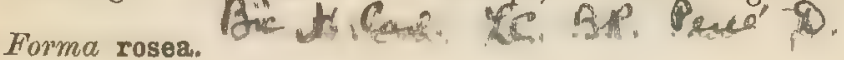

Ray flowers rose to deep rose-red in color. Fréguent. North- $Q_{t} \neq$ east Harbor: Fernald Point road; southern foot of Western Prus $\chi_{\text {. }}$ Mt.; Southwest Harbor, etc. (Rand); - Somesville (R. \& R.).

A. Ptarmica, L. STEEzETORT.

Doubtless an escape, although established for years by road. side spring, far from dwellings, Southwest Harbor (Rand). Adventive from Europe or farther north. 
7. twhenow. H?

B.hypuborea. BR. OR DaR.

B. tripantita Percat

Matrienindiscoutes. C.St, :

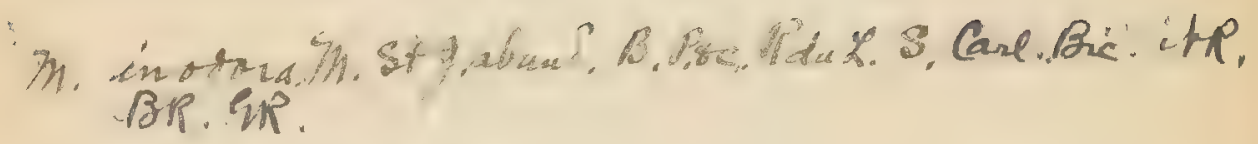

A. farucoos, P. Canet

a.

$s^{*} y t^{+}$

$a$

hate. 


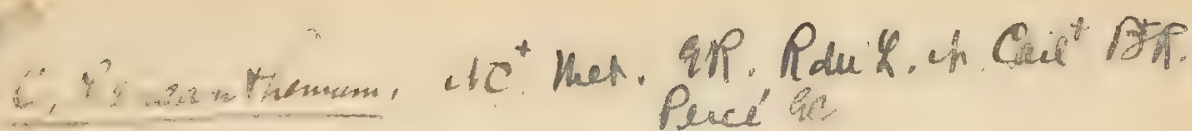

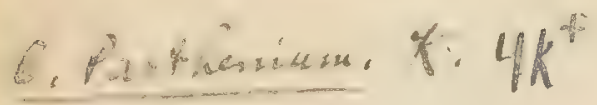

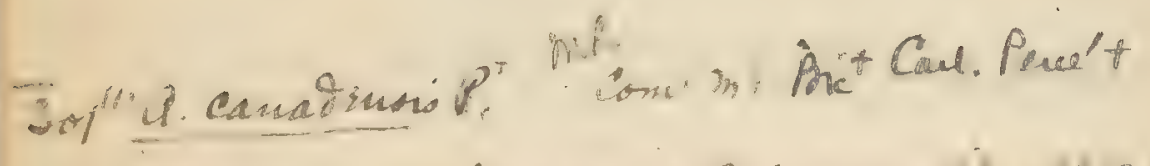

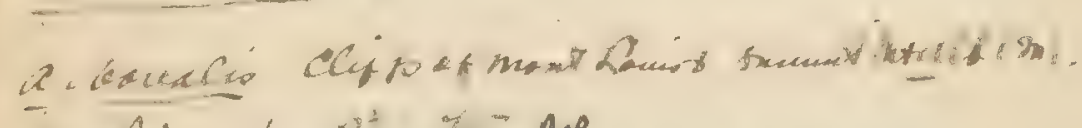

1]. Aicanis Ptc. \%: MP.

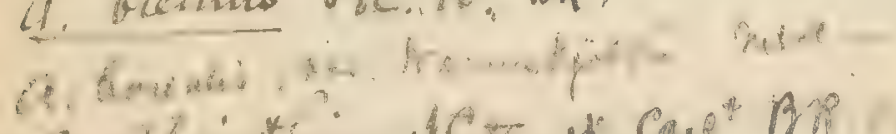

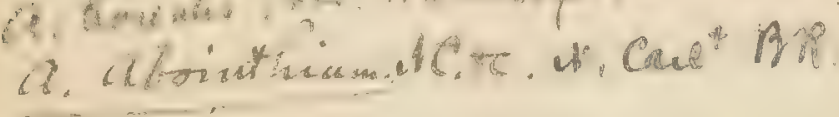

Tusrica, Q. gooff.Stg.jum 27. $4 k^{*}$

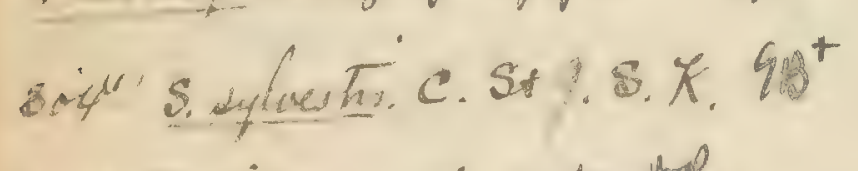

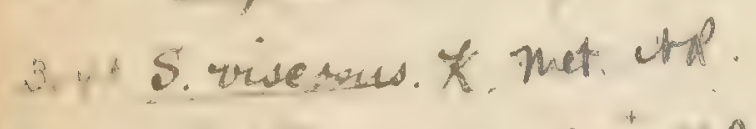

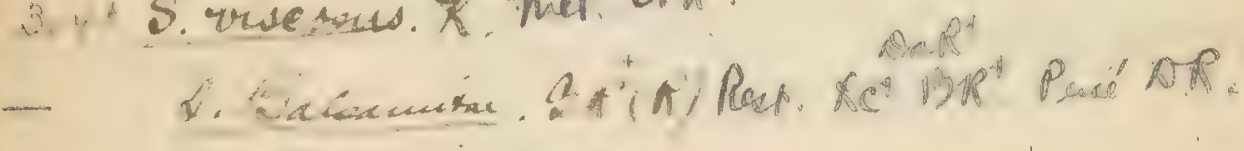

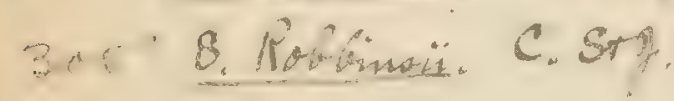

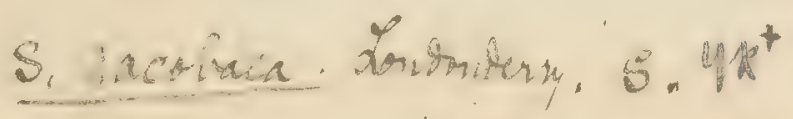

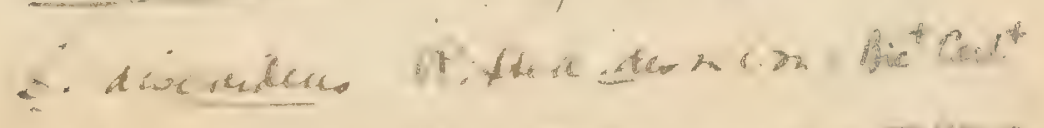

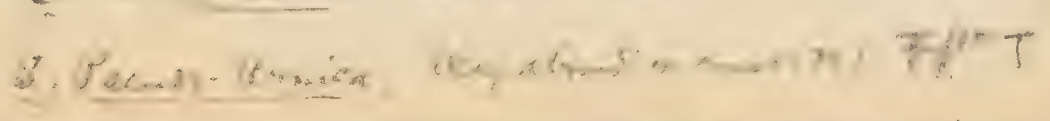


CHRYSANTHEMUM, I. OX-RYE DAISY.

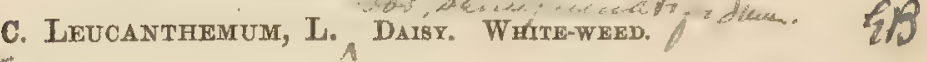

$T$ Fields and meadows; very common. Naturalized from Europe. A form with tubular ray flowers, grens (Rand), $P$,

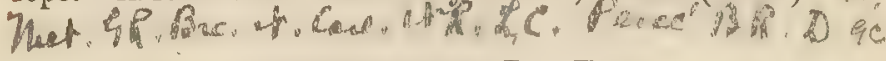

TANACETUM, L. TANSY.

T. vulgare, L. Common Taysy. Gold Buttons. 46

Near old dwellings, and by waysides; frequent: Jordan $B \cdot{ }^{2}$. Pond; Beech Hill; "Sound"; Great Cranberry Isle, etc. B $\mathbb{P}$. (Rand); - Bass Harbor Head; Somesville (R. \& R.); - mouth of Duck Brook (R. H. Day). Naturalized from Europe. C $P$. Piev. U.

ARTEMISIA, L. WORMWOOD.

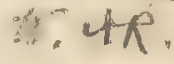

A. VULGaris, L. Mugwort. i : T

Occasional in waste places. West side of Southwest Harbor; $3+6$

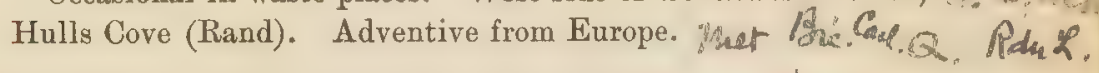

A. Stelleriana, Besser. False Dusty Miller.

Rare. Beach, Mt. Desert Narrows (R. \& R., Annie S.
Downs). Adventive from the north.

PETASITES, Grotn. SWEeT ColtsFoot.

P. palmata (Ait.), Gray. An in fe

Wet ground; rare. Cold Brook (E. Faron, R.\& R.). LC. BP. Pres D.

SENECIO, 工. GROUNDSEL.

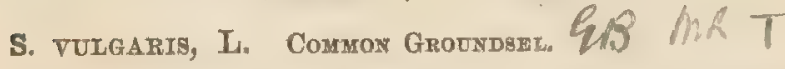

Waste grounds and sea beaches; frequent, especially near $C$. the seashore and on the islands. Also, Green Mt. (Annie S. Atur. Downs). On Flying Mt. is found a low, slender form with cave. aromatic foliage (Rand). Naturalized from Euxope. Whet. $4 \mathrm{R}$,

S. aureus, L. Golden RAGwort.

Rare. Meadow, High Head (Rand). $\mathrm{Cl}^{+} \mathrm{AR}^{+} \mathrm{RC}^{+} \mathrm{BR} \mathrm{P}^{+} \mathrm{Dan}$. ge Pid - 
ERECHTITES, Raf. FIREWEED.

E. hieracifolia (L.), Raf.

Wood clearings, especially in recently burned ground; common.

\section{ARCTIUM, L. BuRdock.}

\section{A. Lappa, L. he h. T} Common in waste places, especially. about old dwellings.
Naturalized from Europe. Har.

CNicus, L. Thistle.

C. tanceoratus (L.), Hoffm. Сommon Thistre. Pencé L/,

Pastures, fields and roadsides; common. Naturalized from $C . \$ 4,0$,

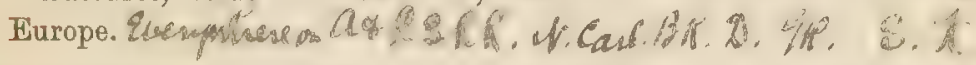

C. arvensis (L.), Hoffm. Canada Turstle. Percé, 0

Pastures, fields, and roadsides; too common. Naturalized $C$.

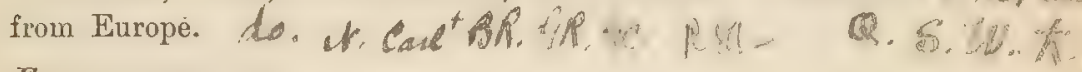
Forma ALBIFLORUS.

Flowers pure white. Southwest Harbor (Rand); - Seal Har- GW, bor (Wm. C. Lane).

\section{Centaurea, L. Star Thistie.}

\section{Cyanus, E: Butebottue. Bachelor's Button.}

Occasionally escaped from cultivation to roadsides and waste places, Southwest Harbor, etc. (Rand, Annie S. Downs).

\section{ARNOSERIS, Gærtn. LamB's Stcoorr.}

A small, annual, scapigerous herb; juice milky; leaves all radical; heads fow, small; peduncles clavate, fistular; involucral bracts in one series, many, after flowering arching over the fruit; receptacle flat, naked, pitted; corollas all ligulate, yellow; anther cells not tailed; upper part. of style and its short obtuse arms hairy; fruit obpyramidal, furrowed and ribbed, not beaked, crowned by a coriaceous angular ring. 


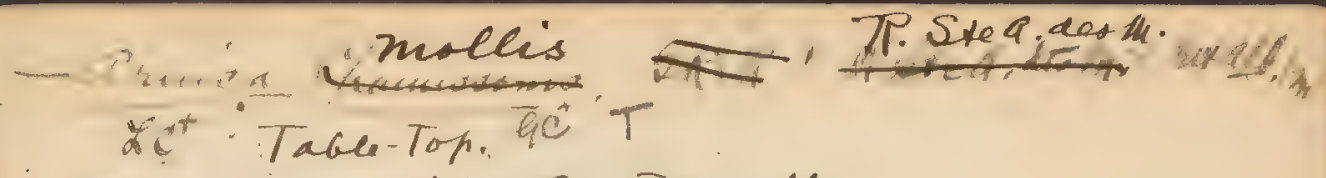

A. gaspenoix. Cas Tourelle

a.Chionophra. ERt Bupt Pereit

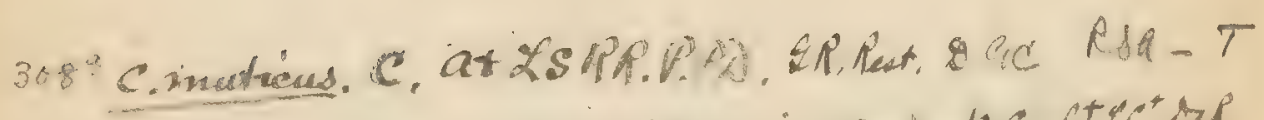

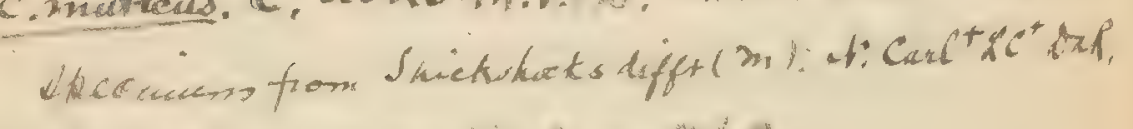

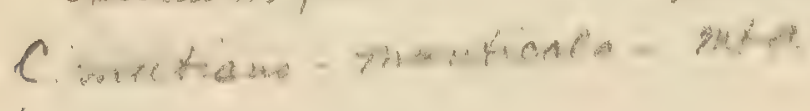

1- E. Beabiona. Eurt

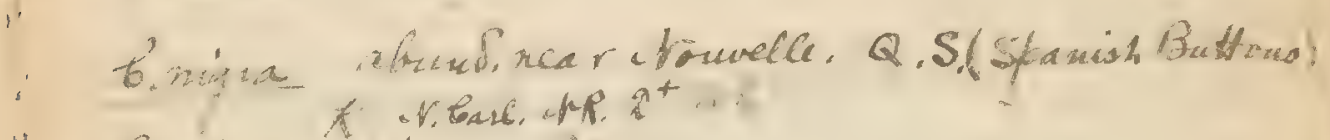

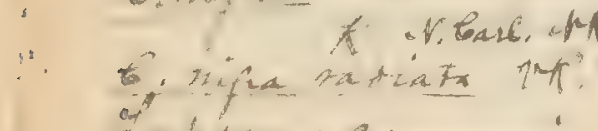

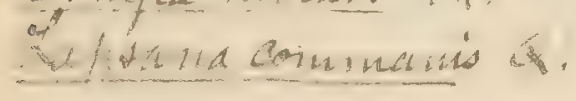




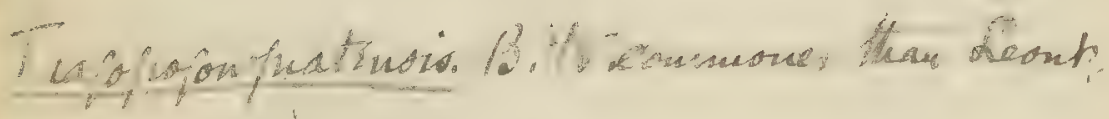
$314 C$. tane

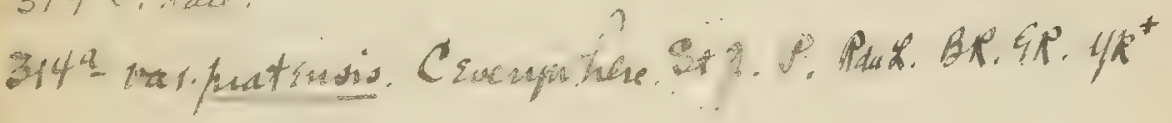
35. Cinstafidiforally

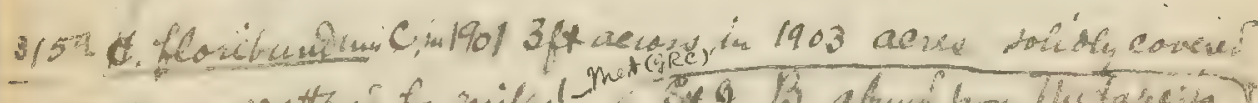

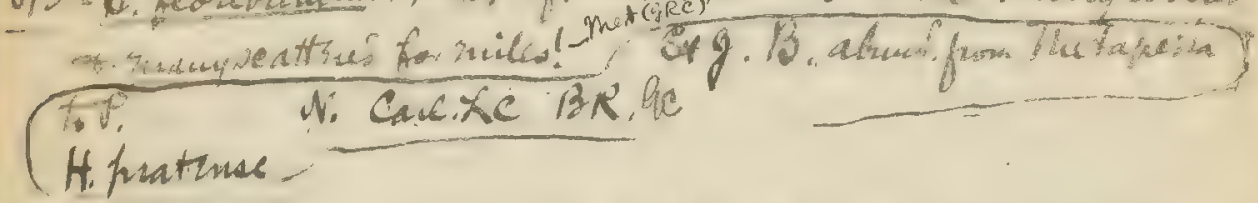

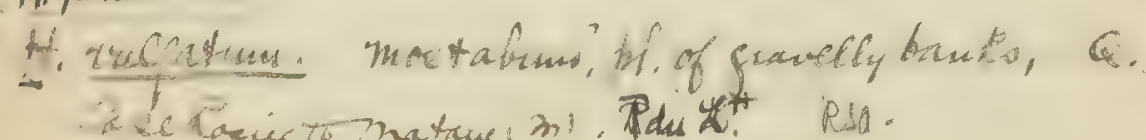

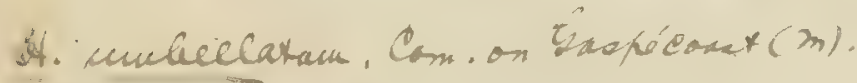

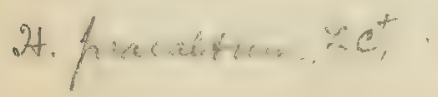


$3 / 2$ - A. pustlia, Grortn.

Glabrous or slightly hairy; heads campanulate, $4^{\prime \prime}$ long, inclined in bud; involucral bracts herbaceous, puberulous, linearlanceolate, tips contracted, obtuse; fruit pale brown, rugose between the ribs; scapes $4^{\prime}-12^{\prime}$ high, many, slender, rigid, sparingly branched above; leaves $2^{\prime}-4^{\prime}$ long, narrow, obovatespatulate or -lanceolate, toothed. Hooker, Fl. Brit. Isles, 229. Rare. Field, Southwest Harbor (M. I. Fernald). Fugitive from Europe.

\section{CICHORIOM, L. CHICORY.}

313 c. Intyius, L. PSh- T EA,

Rare. Formerly in some abundance by roadside, Clark Point, Southwest Harbor (John L. Wakefield, Rand). About 1887 the plant was apparently exterminated in this station. It still persists, however, in another locality on the Point, although in no

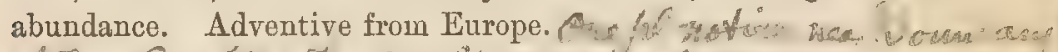

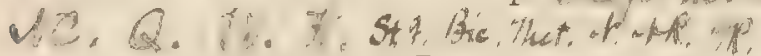

LEONTODON: L. Fali DaNDELion.

\section{AUTUMNALIs, L. 1! $4 k^{*}$}

Fields and roadsides; very common. A form with much aborted ray flowers, Emery District; Southwest Harbor; Great

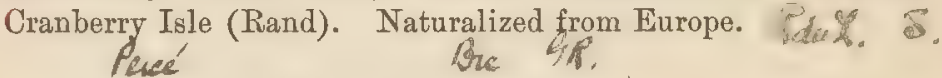
Pevé'

HIERACIUM, L. HAWKWEED.

H. AURantiacum, L. Flaming Hawkwegd.

Fields and meadows; becoming frequent. Beech Hill (R. \& R.); - High Head meadow (Rand); - near Ship Harbor (Faxon \& Redfield); - near Otter Creek (Theodore G. White). Naturalized from Europe.

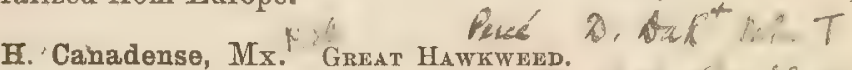

Woods and roadsides; frequent.

H. paniculatum, L.

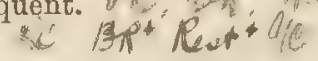

Rare. Clearing on roadside by Denning Pond; east side of of Northeast Harbor (Rand). 
H. scabrum, Mx. RoUgh HaWKWEED. BA. $Z^{*} T$

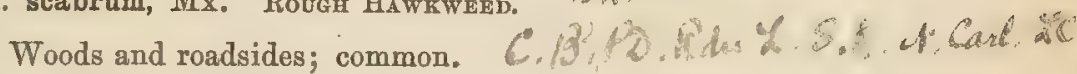

PRENANTHES, L. (Nabalus, Cass.) RATTLESNAKE-Root. serpentania Burs Nabalus Fraseri, DC. C, Q S. $7^{+}$

Dry soil; common.

Var. nana (DC.), Gay. Nabalus nanus, DC.

Common on mountain summits and rocky places. Green Mt. C. R? f (Wm. C. Lane); - Sargent Mt.; Pemetic Mt.; Jordan Mt.;

Beech Cliff; Sutton Island, etc. (Rand). W, wh.... P. altissima, L. Wood Ratrlessake-Root. A $\mathrm{T}$
Rich damp woods; frequent. A form with dark purple his-
pidulous stems, Southwest Valley road (Rand). Xe. the Prax

\section{TARAXACUM, Haller. DANDELION.}

T. officinale, Web. T. Dens-leonis, Desf." Dandelion. "fot

Becoming common; roadsides and waste places, - sometimes $A, P_{0}, 0$

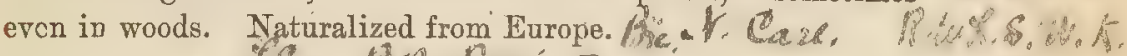

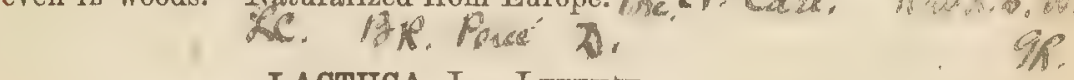

LACTUCA, L. LETTUCE.

L. sativa, I. Garden Lemtuce.

Persistent for years in waste ground, Fernald Point (Rand). Escaped from cultivation.

L. Canadensis, L。 Wind Letrúce.

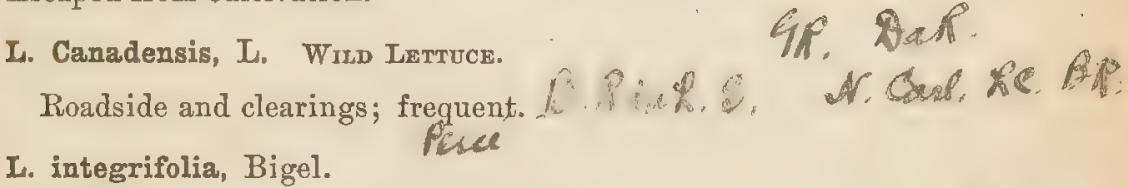

Dry soil; infrequent. Northeast Harbor (John L. Wake L Caul field, Rand);-Somesville; Frenchman Camp (Redfield).

L. leucophæa (Willd.), Gray. Blux Letruce. pela $T$ Low grounds, roadsides, and waste places; common. $A, \mathbb{S}, \mathbb{R}, \mathbb{R}$

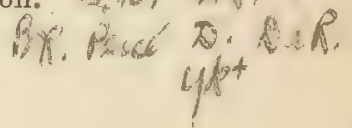




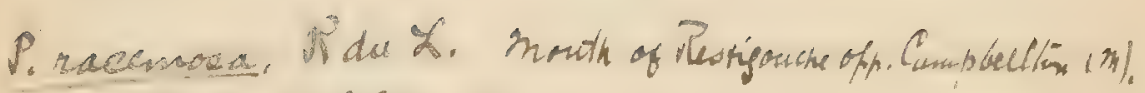
Rest IR ot. AR

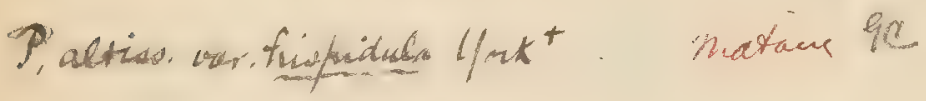

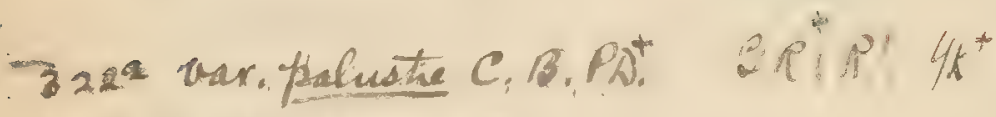
' $322^{\prime}$ isuthardernum $C$.

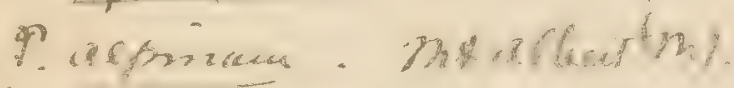

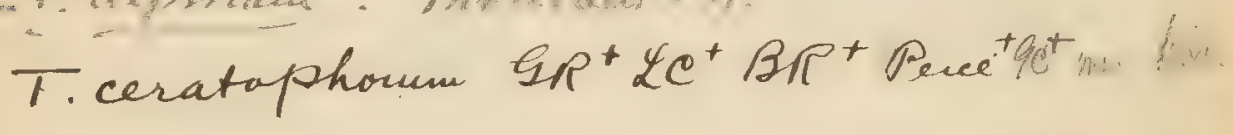

x. 


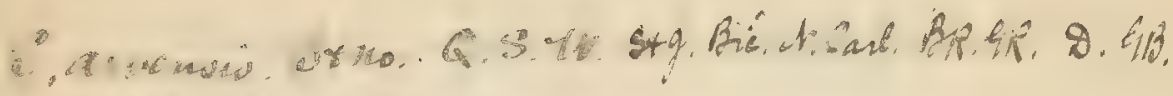
n. is: T

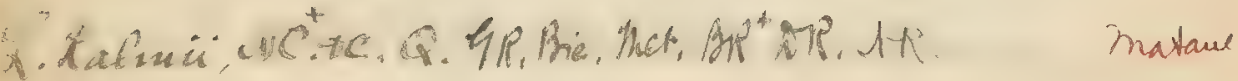


SONCHUS, L. Sow Thistre.

S. oleraceus, L. Common Sow Thistr.x. Waste places; rare. Sawyer Cove; Fernald Point (Rand); - 4 . $4 \mathrm{k}^{\prime}$
High Head (Annie S. Downs); - Clement farm, Seal Harbor (Redfield). Naturalized from Europe. Pace D.

S. Asper, Vill. Sptwy Sow Thustle.

Waste places; very common. Naturalized from Europe.

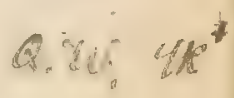

\section{LOBELIACE E. LoBelia Family. \\ LOBELIA, L.}

I. cardinalis, L. Cardinal Flower.

Rare and local. Borders of streams, Somesville and vicinity (Rand and others); - also on brook flowing into Seal Cove Pond (Annio S. Downs).

L. spicata, Lam.

Grassy places; frequent. Somesville; Wasgatt Cove (John L. Wakefield); - fields above Long Pond (Redfield); - Seal Harbor (Sara E. Boggs); - Northeast Harbor; Southwest Harbor (Rand); - "Mt. Desert" (F. M. Day).

L. inflata, L. Indian Toвacco.

Dry fields and roadsides; common. Flowers pale blue, violet, $\vec{C}_{0}$ or whitish.

I. Dortmanna, L. Water LoBelis.

Common on borders of ponds and often of meadow streams. Usually in shallow water; sometimes immersed.

Campanulade fe. Campanula Familt.

SPeCUlaria, Heist. Venus's Looktng-glass.

S. perfoliata (L.), A. DC.

Rare. Dry pasture on road to Mason Point, Somesville (R. \& R.). Possibly introduced. 
CAMPANULA, L. BELLFLOWER.

334 C. RAPUNCULOIDES, L.

Occasionally by roadsides, etc.; escaped from cultivation. $\$ 7 \%, 6 . K$ Oak IIill (Redfield, Annie S. Downs); - High Head; Somesville (Rand). Adventive from Europe. Rie. $1 / \mathbb{K}$.

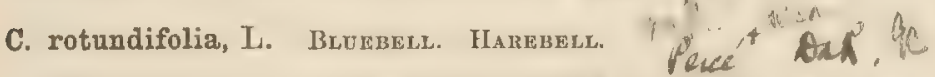
Cliffs on seashore, and frequently on the mountains; common. C. S19.P.Q. Forma albifiora.

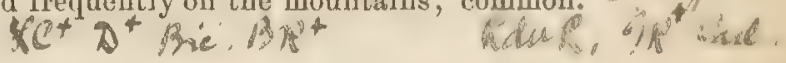

Flowers white. Orens (Annie S. Downs); - Otter Cliffs (Annie S. Downs, Rand).

FricaCe.

GAYLUSSACIA, HBK. HuCKLEBERRY.

337 G. dumosa (Andr.), T. \& G. Boe HuckleBerry.

Frequent in sphagnum bogs. Bog near Somesville (William H. Dunbar); - Somes Pond; Sunken Heath; The Heath, Great Cranberry Isle; Great Heath (Rand).

G. resinosa (Ait.), T. \& G. Сомmox Huckieberrt.

Dry or wet ground; common. C.S.K.

VAccinium, L. Blueberry. Cranberry.

V. Pennsylvanicum, Lam. Dwarf Blueberrx. $2.913 .7 P$.

Very common everywhere in dry soil; abundant on the hills $S R . T$ and mountains. Variable. A form with bluish-red and white fruit, Jordan Mt. (Rand). A well marked form with dark blue- 1.0 .0 . Q green leaves, reddish shoots, and dark blue fruit with little or no bloom, Great Cranberry Isle ( $R_{0} \&$ Ro ). hic.

V. Canadense, Kalm. Cakada Buugberry.

Common in woods or moist ground. Fruit ripening later than that of the preceeding species, and more acid. Biet Gas. in. \&. Ap, or. 
C. Trachelium. Q.

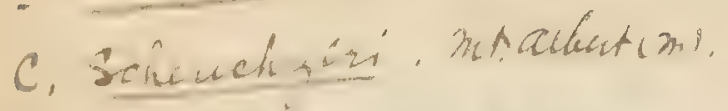

- C. Gonerata Pduxt. Trasi Pistoles

$$
=P^{*}
$$

- $\quad 399$

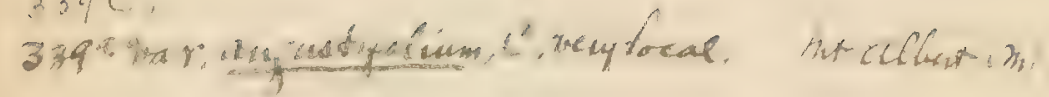
ite. 


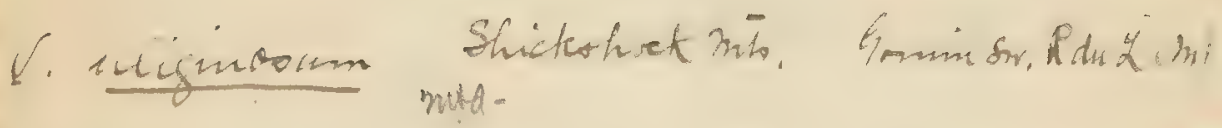

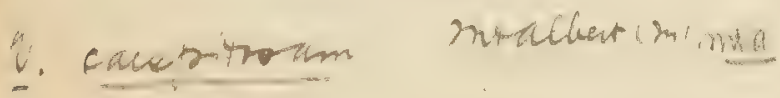

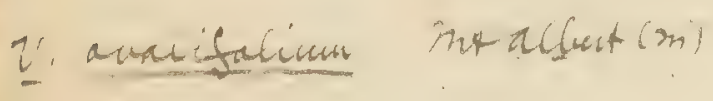

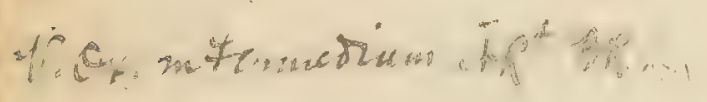

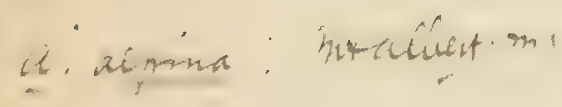


V. corymbosum, L. Hrat-Bush BLUEberry.

Swamps and low thickets; frequent. Somesville and vicinity (R. \& R.); - Witch Hole (Rand); - Hulls Cove (F. M. Day, J. H. Curtis).

Var. amonum (Ait.), Gray.

Somesville (Redfield, M. L. Fernald); - Breakneck Ponds (Rand).

This species and variety seem to be rare except in the central and northern parts of the Island.

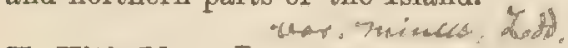

v. Vitis-Idma, td. Mountatx Cranberry. Ear - UK

Common everywhere, shore and mountains, and on the islands. $C$. Fruit much used for sauce, largely taking the place of $V$. macro-

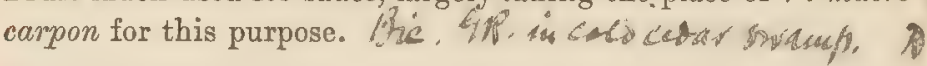

V. Oxycoccus, I. Smatr. Conamberrt.

Common in sphagnum bogs, and in wet places on mountains and shore. Also on Cranberry Isles. or ha $a_{4} \quad \$ f^{t}$

V. macrocarpon, Ait. Large Cramberry.

Bogs; common, but rarely in great abundance. Also on $C, A$. Cranberry Isles, whence their name.

\section{mia chogenes, Salisb. Creeptyg Snowberry.}

C. serpyllifolia, Salisb. C. hispidula (L.), T.\& G. C. $C^{\star}$ Deep mossy woods; common. of Cuse, 70 Xe, Br, flecé An T

\section{ARCTOSTAPHYLOS, Adans. BeARBERRY.}

\section{A. Uva-ursi (L.), Spreng. CoMmon Bé́reernY.}

Open, rocky places; infrequent and local. Browns Mt. (William C. Lane); - Beech Cliff; Dog Mt. (Rand);-Barr $\mathrm{Af}^{f} \mathrm{~B}_{\mathrm{ie}}{ }^{\dagger}$ Hill; Newport Mt. (Redfield); - "Somes Sound, Southwest Harbor" (Elizabeth G. Britton); - Robinson Mt. (Anna H. Bee); - near Bar Harbor (W. H. Manning). Bic $m+4$. 
EPIG Ėa, L. Trailing Arbutus.

E. repens, L. Mapflower. Traiting Arbutus. Ma -

Frequent in woodlands, but seldom very abundant. $S \times \varphi^{t}$

GaULTHERIA, L. Aromatic Wintergreen.

G. procumbens, L. Creeping Wintergregn. Checkerberry,

Common everywhere in woods, shady places, and clearings. $C, A^{+}+T^{+}$

ANDROMEDA, L.

A. pelifohis, J.

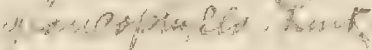

Bogs; infrequent. Swampy roadside, south of Salisbury Cove; Hadlock Upper Pond; Sunken Heath (Rand);-Pond Heath (Greenleaf, Lane \& Rand); - Great Heath (Redfield).

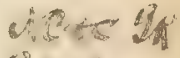

CASSANDRA, Don. Leather Leaf. at $\mathrm{MP}^{+2}$

C. calyculata (L.), Don. ctit. \& 27

KaLMIA, L. American LaUrel.

K. angustifolia, L. Sheep Latrex. Lambill. hia -

Hillsides, pastures, and thickets in dry or damp ground; $C$ C

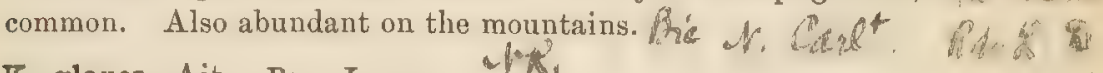
K. glauca, Ait, Pade Latrez. of

Sphagnum bogs; frequent. Pond Heath (Greenleaf \& Rand, E. Faxon); - Freeman Feath (Faxon \& Rand); - Sea Wall Swamp; Sunken Heath; Great Heatlı (Rand); - bog by roadside west of Sea Wall; The Heath, Great Cranberry Isle (R. $\&$ R.).

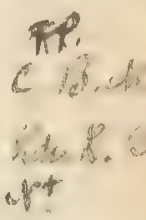

RHODODENDRON, L. ROSE-BAY. AZALEA.

R. Rhodora, Don. Rhodora Canadensis, L. RHodorA.

Common in damp thickets and swamps, and in wet or even $6,3,5$ in dry places on the mountains. Very variable in color of flowers. Sometimes three to five feet high. 


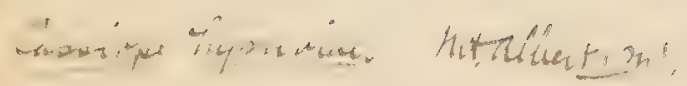

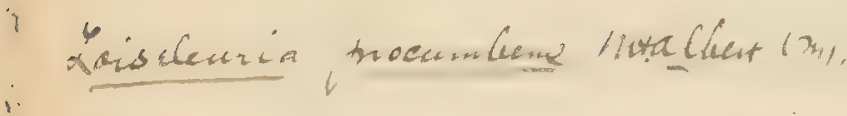

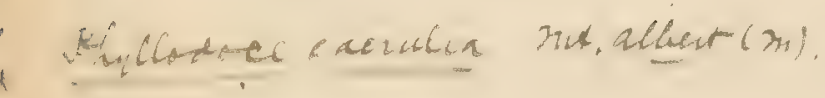

R. Lathonicam Prathert (m). 


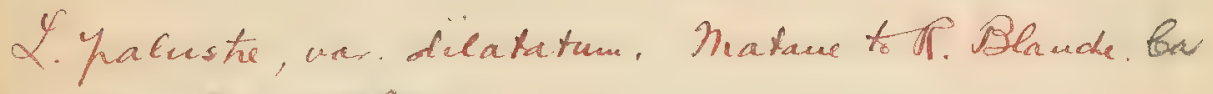
B. F. mita-

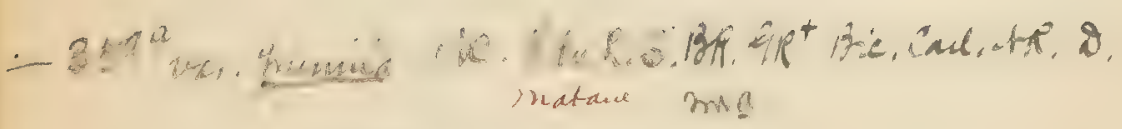

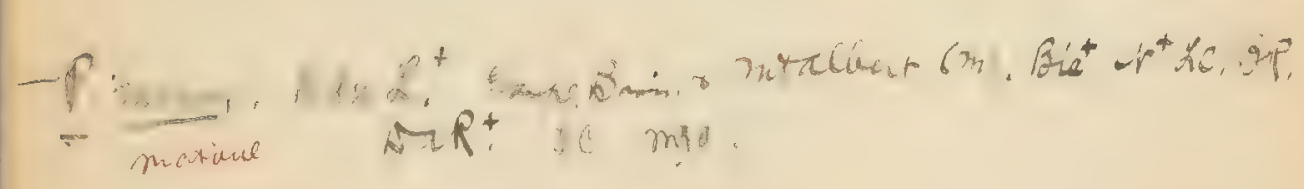




\section{Forma albiflora.}

Flowers pure white. Southwest Harbor (Annie S. Downs).

\section{LEDUM, L. Labrador Tea.}

\section{L. latifolium, Ait.}

Common in bogs, and often in dry ground. Not very abundant in the southern part of the Island, but common on the

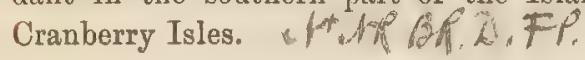

\section{CLETHRA, L。 WHITE ALDER.}

$3 \sqrt{6}$

C. alnifolia, L. Sweet Pepperbugh. White Alder.

Rare. Wet ground near Hadlock Upper Pond (Annie S. Downs). Reported to grow also in meadow on Denning Brook, and on Great Cranberry Isle.

\section{Chimaphila, Pursh. Wintergreen.}

C. umbellata (L.), Nutt. Prince's Prne. Pirsissewa. Winter ge. $\$$

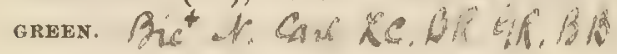

Dry woods; frequent.

\section{MONeSES, Salisb. One-Flowered Pyrola.}

M. grandiflora, Salisb. M. uniflora (L.), Gray. Thataue

Deep mossy woods all over the Island; frequent. Also on the $C$.

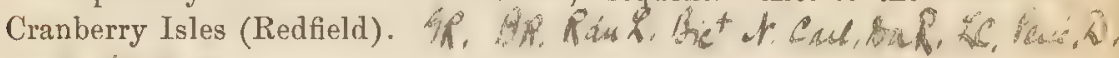
math $l_{1}, i, \ldots l 6$

\section{PYROLA, L. Shivimar.}

P. secunda, L. One-sided Prrola. Verié

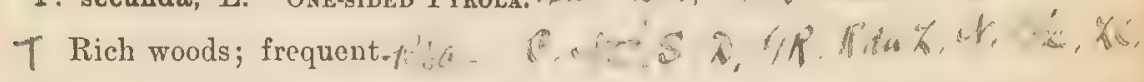

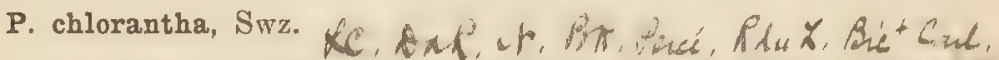

- Deep woods; infrequent. Little Harbor Brook Notch; Cold Brook (Rand); - Bar Island, Bar Harbor (F. M. Day); - Seal Harbor (Redfield); - near Beech Hill (Arnold Greene). pesa 
P. elliptica, Nutt. it:

Common in woodlands.

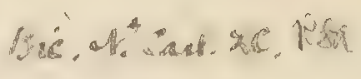

ancriequa, suse $x$.

P. eatromafalio,n. Round-Leaved Pyroxa.

Occasional in dry woods. Great Pond; Salisbury Cove (Henry C. Jones); - Seal Harbor (Redfield); - Cold Brook (Rand).

monotropa, L. Indian Pipe, Pinesap.

M. uniflora, L. Indiax Pipe.

Damp woods; not uncommon.

C.S Bie.culigtifese D. Ha

M. Hypopitys, L. Pinesar.

Dry woods; infrequent. Hadlock Upper Pond; eastern side thé Cod: of Browns Mt. (William H. Dunbar); - Cold Brook (Rand); $4 f$, Green Mt. Gorge (F. M. Day); - Western Mt. (Annie M.

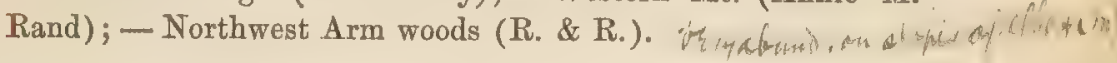

\section{PLUMBAGINACE E. Leadwort Familit.}

Statice, I. Marsh Rosemary.

S. Limonium, L., var. Caroliniana, Gray. Sea Lavender.

Muddy beaches and salt marshes ; frequent. Somes Harbor, $:$ and shores at head of Somes Sound (Henry C. Jones, R. \& R.); - near Orens; Bass Harbor; High Head, and northern shores of the Island (Rand); - Great Cranberry Isle (R. \& R.). Bie

\section{PRimulace E. Primrose Family.}

TRIENTALIS, L. STAR-FLOWTR.

Dak. BR. Fecé D. YK. Bie.et. T. Americana (Pers.), Pursh. Star-flower. STar Anemone. C B a la Low woods; common. $g \mathrm{C}$,

LYSIMACHIA, L. LOOSESTRIFE.

L. quadrifolia, L. WhorLed Loosestrife.

Open woods, hills, and roadsides; common. 


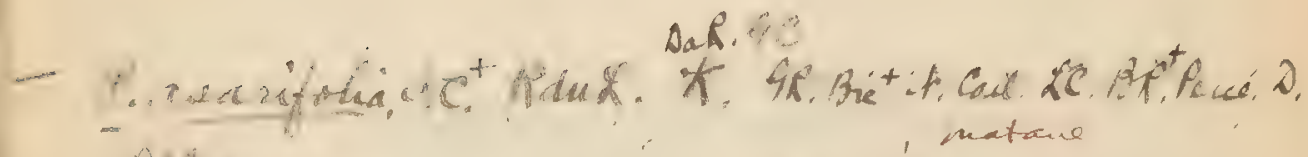
pu-

, matare

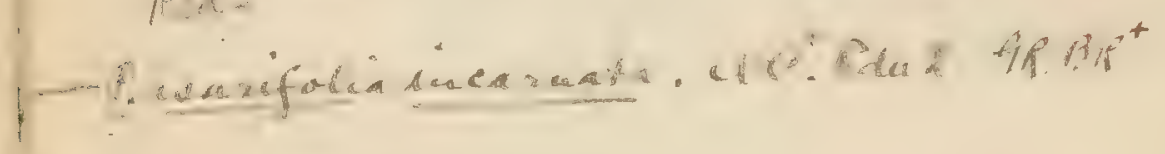

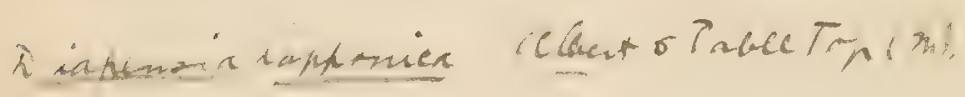

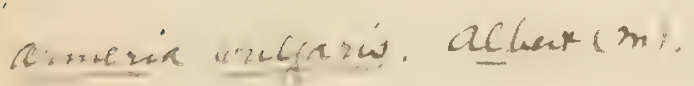

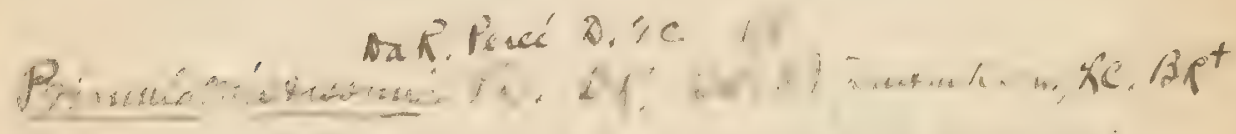

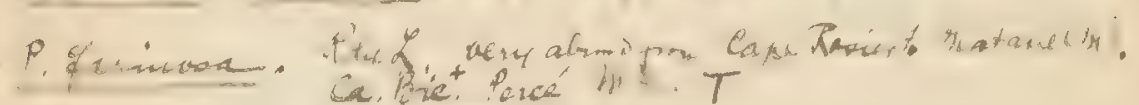

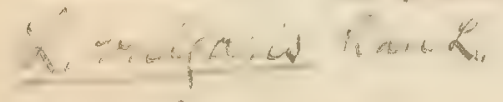


sous masirina. D. W.

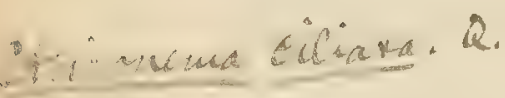

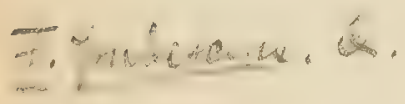

- 2. madium, Rett $4 l^{2}$ in

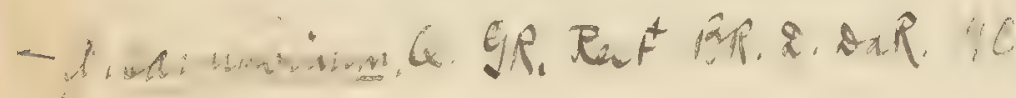




\section{CATALOGUE OF PLANTS. \\ Ratu, Aar, $d$}

\section{L. stricta, Ait. Swamp Loosestrifw.}

Wet ground and swamps; common.

Var. ovata.

Stems at length much branched above, the branches often equalling or exceeding the inflorescence; leaves ovate to ovatelanceolate, narrowing less abruptly at the base, shorter-petioled, veins apparent; raceme short and few-flowered. Wet ground, Somesville (Redfield).

I. thyrsiflora, I. Tueted Loosestrife.

Rare. Intervale Brook, near Hulls Cove (F. M. Day); - a bog, northern foot of Beech Hill (Rand).

GLAUX, L. ' Sta Milkwort.

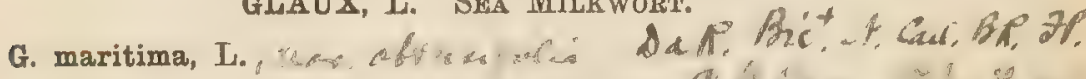
Salt marshes and muddy beaches; frequent. $C ; t<$. T

\section{OLEACEAE. OLIVE Family.}

FRAXINUS, L. Ash.

F. Americana, L. Wuтte AsH.

Moist woods; common.

a. 1

F. sambucifolia, Lam. Busck Asr.

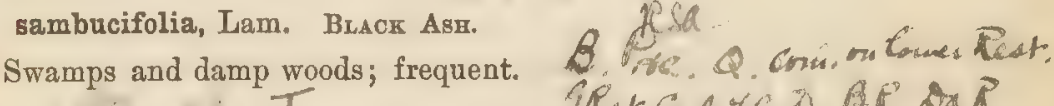

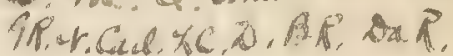
SYRINGA, L. LILAC.

S. vUlgaris, I. Common litac.

Occasionally escaped to roadsides near dwellings (R. \& R.). " Adventive from Eastern Europe or Asia. inff, nuct. Jusse 28

\section{aPOCYNACE}

APOCYNUM, L. DogBane.

A. androsæmifolium, L. Spreading Dogbane.

Roadsides and thickets; infrequent. Otter Creek (William (_. Af H. Dunbar); - Northeast Harbor, and elsewhere (Rand); -

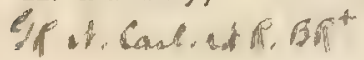


Prettymarsh (Redfield);-Somesville; Hadlock farm, Seal Harbor (R. \& R.) ; - Bar Harbor (F. M. Day).

\section{GENTIANACEE. Gentian Family.}

\section{BARTONIA, Muhl.}

\section{B. tenella, Muhl.}

Rare. Damp hollows, summit of Green Mt. (Rand, William C. Lane).

\section{MENYANTHES, I. BuCKBEAN.}

\section{M. trifoliata, I. Pra TaR. HC T}

Bogs; rare. Northeast Harbor (John L. Wakefield); - Somes C. AC C Pond (Rand); - Great Duck Island (Redfield); - Mt. Desert

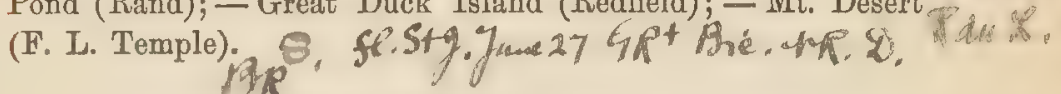

\section{limnanthemum, Gmel. Froating Hrart.}

L. lacunosum (Vent.), Griseb.

Frequent in ponds. Hadlock Lower Pond (William H. Dunbar); - Witch Hole (Rand, F. M. Day, Redfield);-Ripples Pond ; Great Pond; Denning Pond (Rand);-Eagle Lake ; Mountain Pond; Newport Pond (Redfield).

\section{BORRAGINACEAE. Borage FAMiLT.}

MERTENSIA, Roth. LUNGWORT.

M. maritima (L.), Don, SRA Lungwort. R. Blauche $T$

Frequent on sea beaches, especially on southern coast of the $C, 3, a_{\text {. }}$ Island, and on the Cranberry Isles. Flowers pink to blue, very rarely white. Bic Cal? Bar. D. If . in:

\section{LYCOPSIS, L. BUGLOSS.}

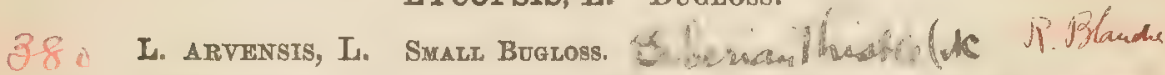

Naturalized for years at Fernald Point (William H. Dunbar, M Rand); - also in waste ground, Somesville (Rand). Naturalized from Europe.

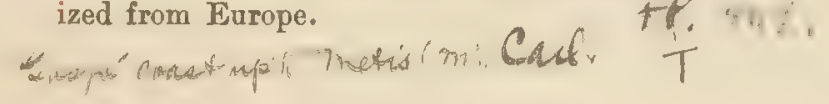




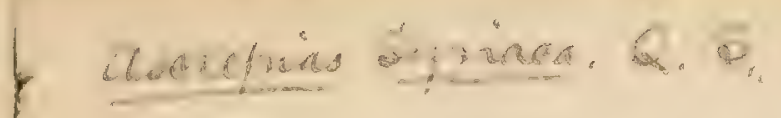

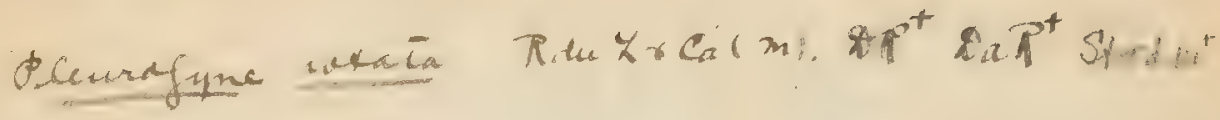

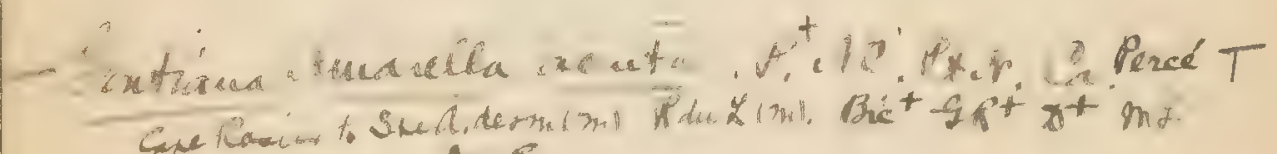
tak

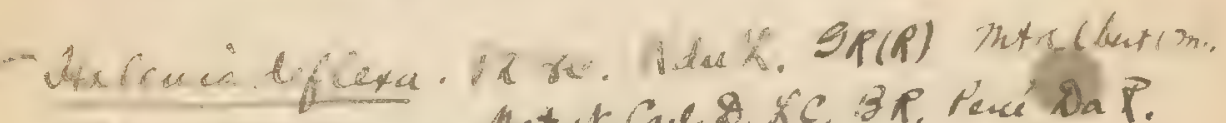

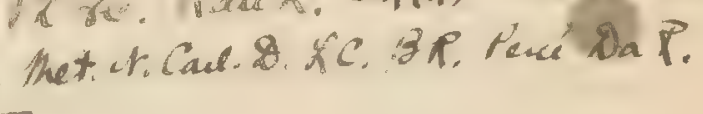

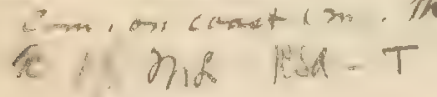

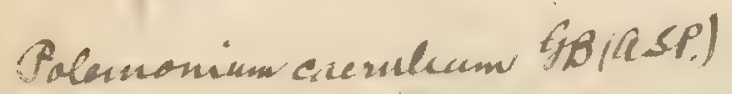

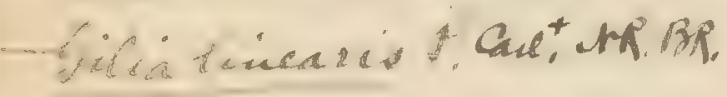

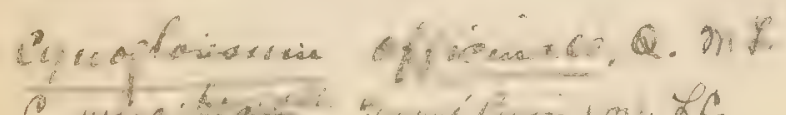

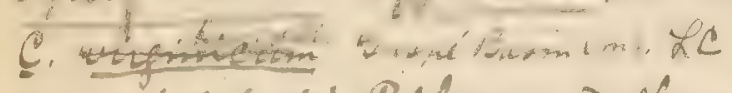

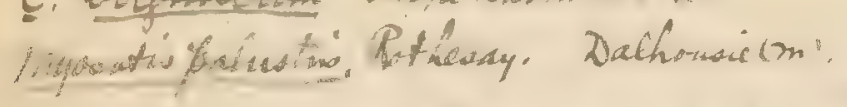

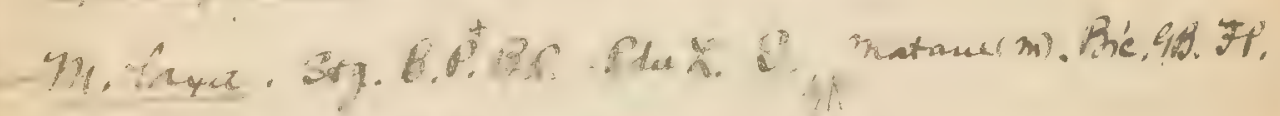

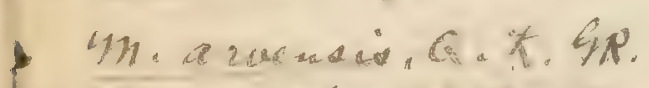

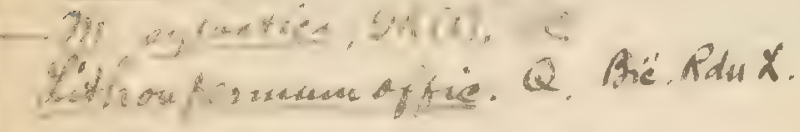

Eumphoptum - - To. Bie

Q. roperimume Qxaburt?

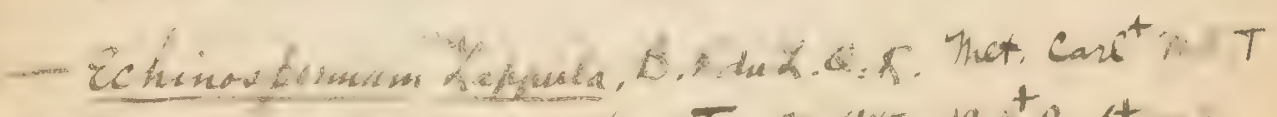

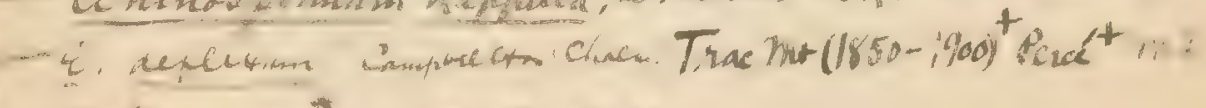

Cherm trafan arderti! 
c. I: thesis \& lpt.

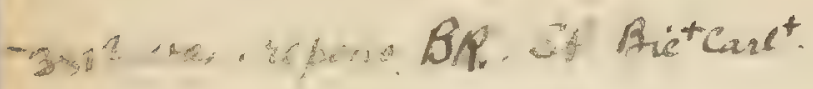

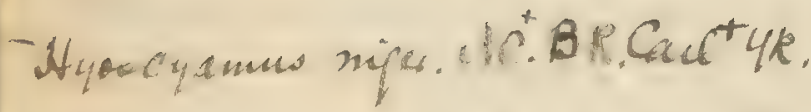

- Phyoain qrantifiona Bret 
CONVOLVULACEAE. Convolvulus Family.

CONVOLVULUS, L. BINDWEED.

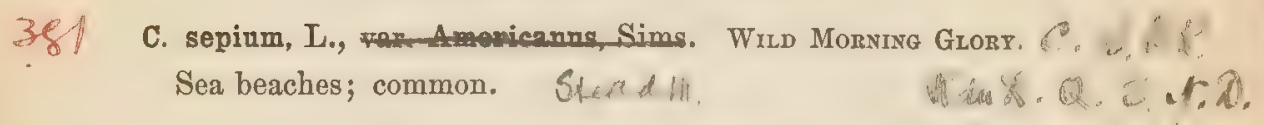

CUSCUTA, L. DODDER.

382 c. Gronovii, Willd.

Infrequent; mostly on the coast. Growing on various $\%$ plants, especially Aster, Solidago, and Ligusticum. Baker Island (Henry C. Jones, Redfield); - Southwest Harbor; Somesville; Sea Wall, etc. (Rand).

SOLANACE $\bar{A}$. Nrghtghade Family.

SOLANUM, I. NIGHTSHADE.

S. Dulcamara, L. Bitrerawert.

Near dwellings and in low grounds. Hulls Cove; Breakneck , Road (F. M. Day);-Bar Harbor (W. H. Manning). Adventive from Europe.

S. nigrum, L. Common Nightshade.

Frequent on sea beaches, seldom elsewhere. Cranberry Isles (John L. Wakefield, R. \& R.) ; - Greening Island; Mill Cove; Bar Harbor; Sea Wall, etc. (Rand). Probably not indigenous within our limits.

\section{NiCANDra, Adans. Apple of Prev.}

N. PHYsaloides (L.), Gritn.

Waste ground, foot of Long Pond (R. \& R.). Adventive from South America.

\section{SCROPHULARIACEAE. Figwort Family.} VERBASCUM, L. MUlleiN.

V. Thapsus, L. Common Molrens.

Fields, pastures, and roadsides; frequent. Naturalized from

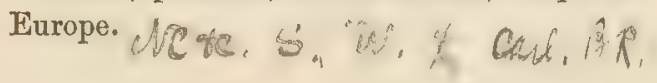




\section{IINARIA, Juss. TOAD FuAx.}

L. Canadensis (L.), Dumont. Wird Toad Flax.

Frequent in dry soil. An exceedingly depauperate form in 6. gravelly hollows among rocks, especially on the mountains. Browns Mt.; Flying Mt., etc. (Rand); - shore, Northeast Harbor (B. E. J. Gresham); - Baker Island (Redfield).

L. vulgaris, Mill. Butter-ANd-Eggs.

Roadsides; infrequent. Southwest Harbor; Town Hill; $Q Q$ Q. $/$ Great Cranberry Isle (Rand); - Baker Island (Redfield). $/ f$. , Calt $^{+}$

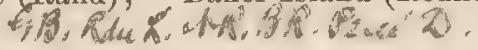

CHELONE, L. SNakthead. Turtle-head.

C. glabra, L.

Wet places, along brooks and rills; frequent. $1 C+Q, Q, i, \ldots l:$

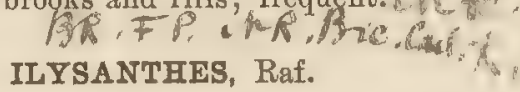

I. riparia, Raf. I. gratioloides (L.), Benth. False Pumpernel.

Rare. Muddy border of Somes Stream (R. \& R.); - shore of little mill-pond, Somesville (Rand).

\section{VERONICA, L. SPEEDWELL.}

V. scutellata, L. Marsh Speedweli.

Boggy ground; infrequent. Northeast Harbor (William H. C. AR. Dunbar); - Ripples Pond; High Head meadow; bog near Sea ihet $D$ Wall (Rand); - "Mt. Desert" (F. M. Day).

V. officinalis, L. Common Speedwell.

Dry ground; rare and local. Roadsides and fields, Salisbury C.C.5. Cove (Faxon, R. \& R.); - "Norway Drive," south of Salisbury y Cove (Rand, Mary Minot). Apparently confined to the neighborhood of Salisbury Cove, and appearing both introduced and indigenous.

V. serpyllifolia, L. Thyme-teated Speedwell. Peć́

Fields, clearings, and roadsides; common. Apparently both introduced and indigenous. $9 R^{3}+3,2, A R, A R, A R$. $\overline{i c}$ 


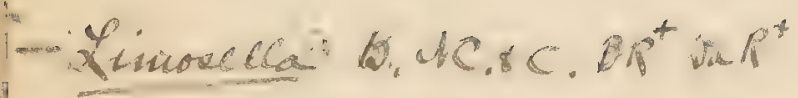

If Lonfifolie. t.

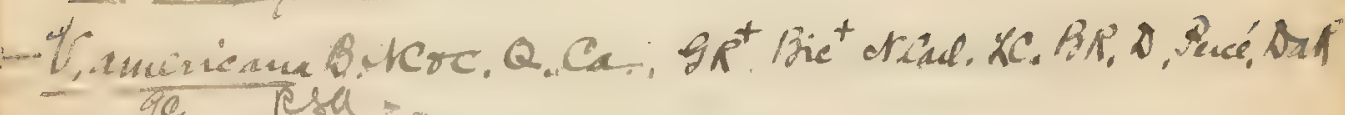

L. Bescofouga, Q.

H. Q Q

V. Sinchirinï Q.

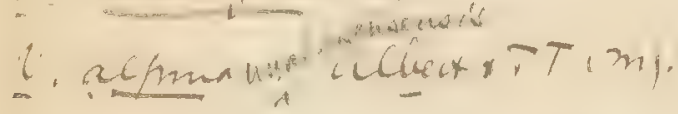

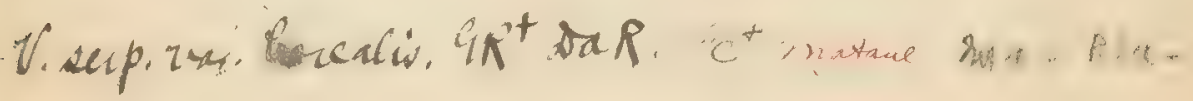




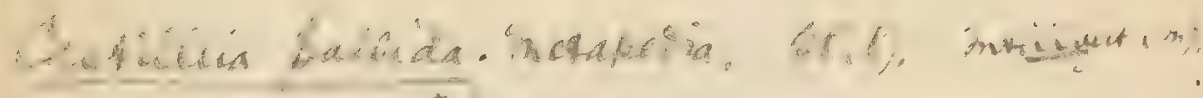
Test. BR, Pisé liet

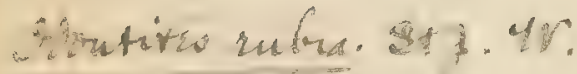

Yision agrestis, if

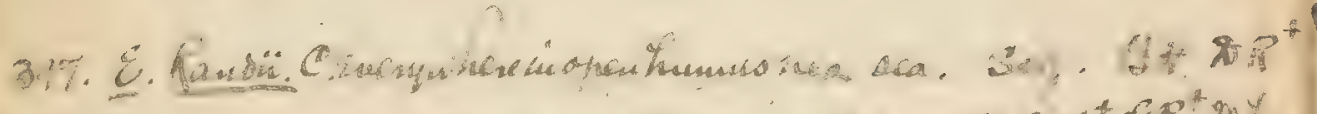

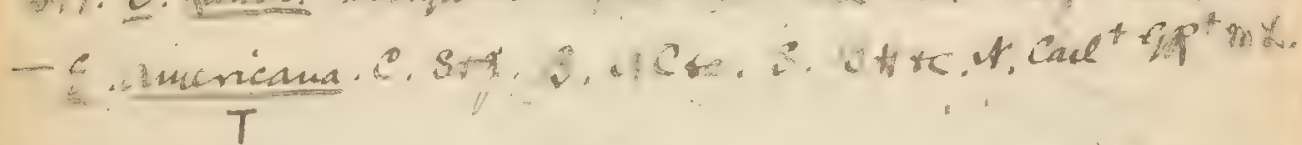

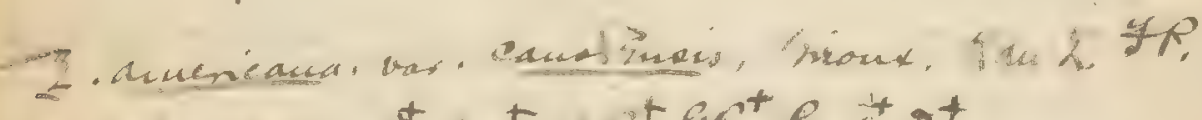

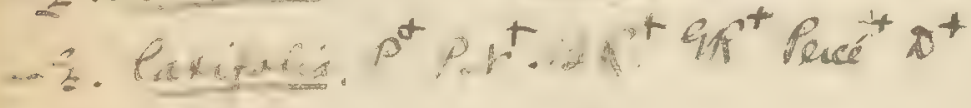

E. 2. Mors6. Ame* 
V: peregrina, L. Purguare Sperdwell,

Dry places; frequent. Flying Mt. (Henry C. Jones); - Norwood Cove, etc. (Rand); - Seal Harbor; Great Cranberry Isle; Great Duck Island (Redfield); - Somesville (M. L. Fernald); - mouth of Denning Brook (R. \& R.). All plants small and dwarfed, hardly branched, very unlike the common garden form of this weed.

V. ARVENSis, L. Corn SPEedwell.

Dry places; infrequent. High Head; Flying Mt. (Rand); $-\mathbb{C}^{d}$ Little Cranberry Isle (Redfield); - mouth of Denning Brook (R. \& R.);-Bar Harbor (Dr. H. C. Chapman). Appearing indigenous here, but said to be naturalized from Europe.

v. Buxbaumit, Ten.

Waste ground; rare. Norwood Cove (Rand). Adventive $g^{+}$ from Europe.

\section{EUPHRASIA, L. EyebRIGHT.}

E. officinalis, L.

Dry ground; common in the southern part of the Island, and on the neighboring islands. Also Bar Harbor (W. H. Manning). If introduced, of very early introduction, but probably indigenous. Very variable. A form from Sea Wall (Rand), having extremely small flowers with corolla scarcely spreading, and leaves less toothed and cut and more crenate, corresponds fairly well to the description of var, Tatarica, Benth., but does not agree with herbarium specimens. It appears to be intermediate between $E$. curta, Fries, and $E$. gracilis, Frios. The species, however, is so variable, and has been so subdivided, that it is impossible to name with any certainty its subspecies and varieties without a careful study of the type specimens.

\section{RHINANTHUS, L. YeLLOW RATTLH.}

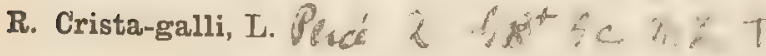

Common in fields and on roadsides. If introduced, of very $C, A$ early introduction.

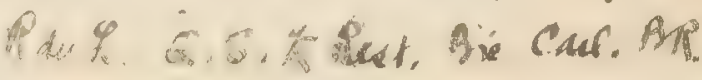




\section{PEDICULARIS, L. LOUSEWORT.}

P. Canadensis, L. Common Lousewort.

Fields; common in centre, west, and north of the Island; rare elsewhere, e. g. Beech Hill (R. \& R.) ; - Northeast Harbor; Southwest Harbor (Rand).

MELAMPYRUM, L. COW WHEAT.

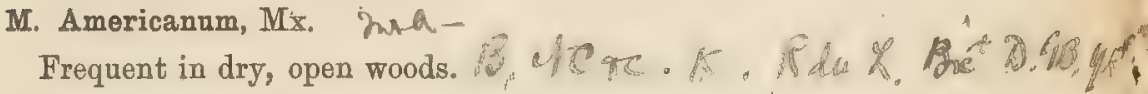

\section{OROBANCHACE 2 E. BRoom-Rape Family.}

EPIPHEGUS, Nutt. BEECHDROPS.

$40 /$ E. Virginiana (L.), Bart.

Under beech trees; rare. Northern end of Jordan Pond (Redfield);-Clark Valley (Rand).

\section{APHYLLON, Mitchell. Naked Broom-rape.}

A. uniflorum (L.), Gray. ONE-hlowtered Broom-Rape.

Low ground, woods and copses; rare. Near Little Harbor; 3 , head of The Barcelona meadow (Rand). Bic

\section{LENTIBULARIACE E. Bladderwort Family.}

UTRICULARIA, L. BLADDERWORT.

U. inflata, Walt. Floating Bladderwort.

Rare. Witch Hole (Rand, F. M. Day, Redfield).

ర. clandestina, Nutt.

Rare. Mountain Pond (Rand); - pools, west side of Great Cranberry Isle (R. \& R.).

U. vulgaris, L. Greater Bladderwort.

Marshy ponds, pools, and slow streams; common. S. WR 


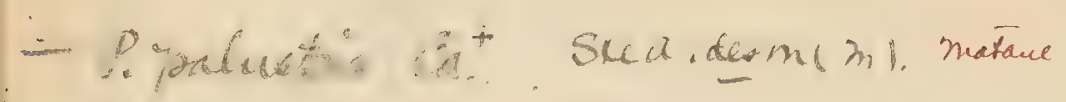

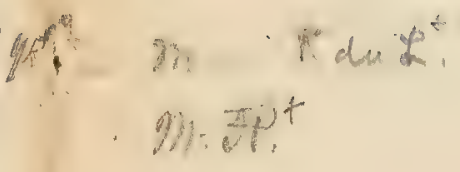




$$
11 . \min \operatorname{tin}^{+}
$$

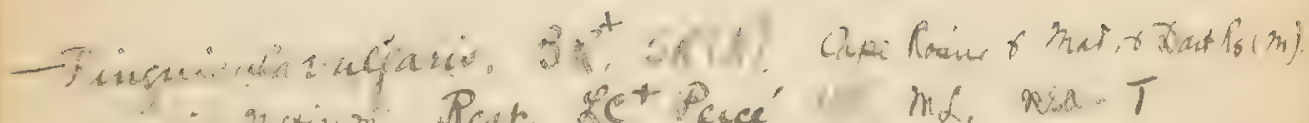

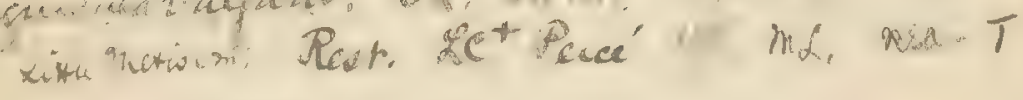

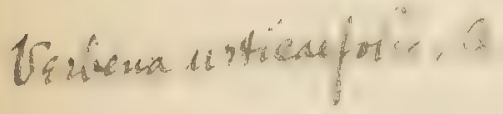


406 U. gibba, L.

Pond shores; rare. Breakneck Ponds (F. M. Day); - Somes Pond (Rand). A form from mud flats, Somes Pond (Rand), closely approaches $U$. biflora, Lam., and may perhaps prove to be that species. "The flower has the spurs of $U$. biflora very decidedly, but the foliage and the bladder's are those of U. gibba. The spur here is oblong, narrow, not curved but projecting straightwise, and the perianth is somewhat larger than is generally the case in $U$. gibba. Other specimens with foliage and bladders better represented might show this to be $U$. biflora, but at present it is safer to call it ' $U$. gibba verging towards U. biflora in flowers.'" Dr. Thomas Morong in litt.

407 U. intermedia, Hayne.

Bogs and streams; , common. Usually sterile; but in flower, Breakneck Ponds (R. \& R., E. Faxon).

U. purpurea, Walt. Large Purple Bladderwort.

Ponds; infrequent. Seal Cove Pond (R. \& R.);-Aunt Bettys Pond (Rand).

\section{U. resupinata, B. D. Greene. Smalt Purple Braddermort.}

Pond shores; rare. Breakneck Ponds (F. M. Day);-Ripples Pond (M. L. Fernald).

U. cornuta, Mx. Long-SpUrred Bladderwort.

Very common on pond shores, in marshes, and sphagnum

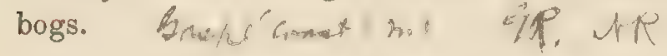

\section{I.ABIAT A. Mint Family.}

\section{TEUCRIUM, L. Germander.}

T. Canadense, L. Amertoan Germander. Wood Sage.

Infrequent on banks and in low ground by the shore, at the head of sea beaches. Long Pond (William L. Worcester); Duck Cove (Rand); - Seal Harbor (Redfield); - Southwest Harbor (Annie S. Downs); - Otter Creek (R. \& R.). 
MENTHA, L.' MrNT.

M. VIRIDIs, L. Spearmint.

Rare. Runlet and roadside ditch, Seal Harbor (Redfield); $-\mathbb{\infty}$ head of Ripples Pond (Rand). Naturalized from Europe.

M. satrva, L. Wronted Mint. (Canhaca) Capelineto

Brooksides; rare. Northeast Harbor; near Carter Nubble t. (Rand) ; - Northeast Creek (M. I. Fernald). Naturalized from Europe.

M. Arvensis, L. Conn Mint.

Roadside ditches and moist ground; infrequent, but not rare about Southwest Harbor. Also Great Cranberry Isle (R. \& R.); - field above Long Pond (Rand). Naturalized from Europe. A tall form with thinner, more sharply serrate leaves, wet roadside by Juniper Cove; broolsides, Norwood Cove (Rand).

\section{Canadensis, L, Wrid Mrxx.}

Wet places and shady banks; frequent. Also Great Cranberry Isle (Rand); - Little Cranberry Isle (Redfield). '? anch.
XC. ER. Precé
D. Aan. $1: a$ IYCOPUS, I

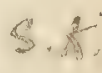




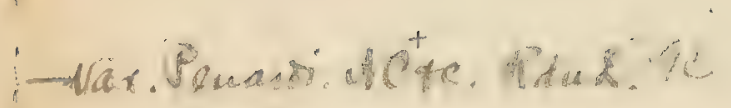

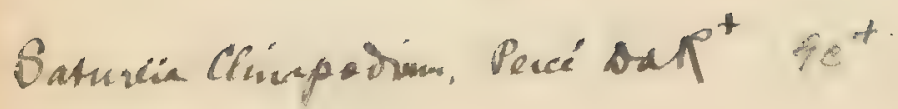




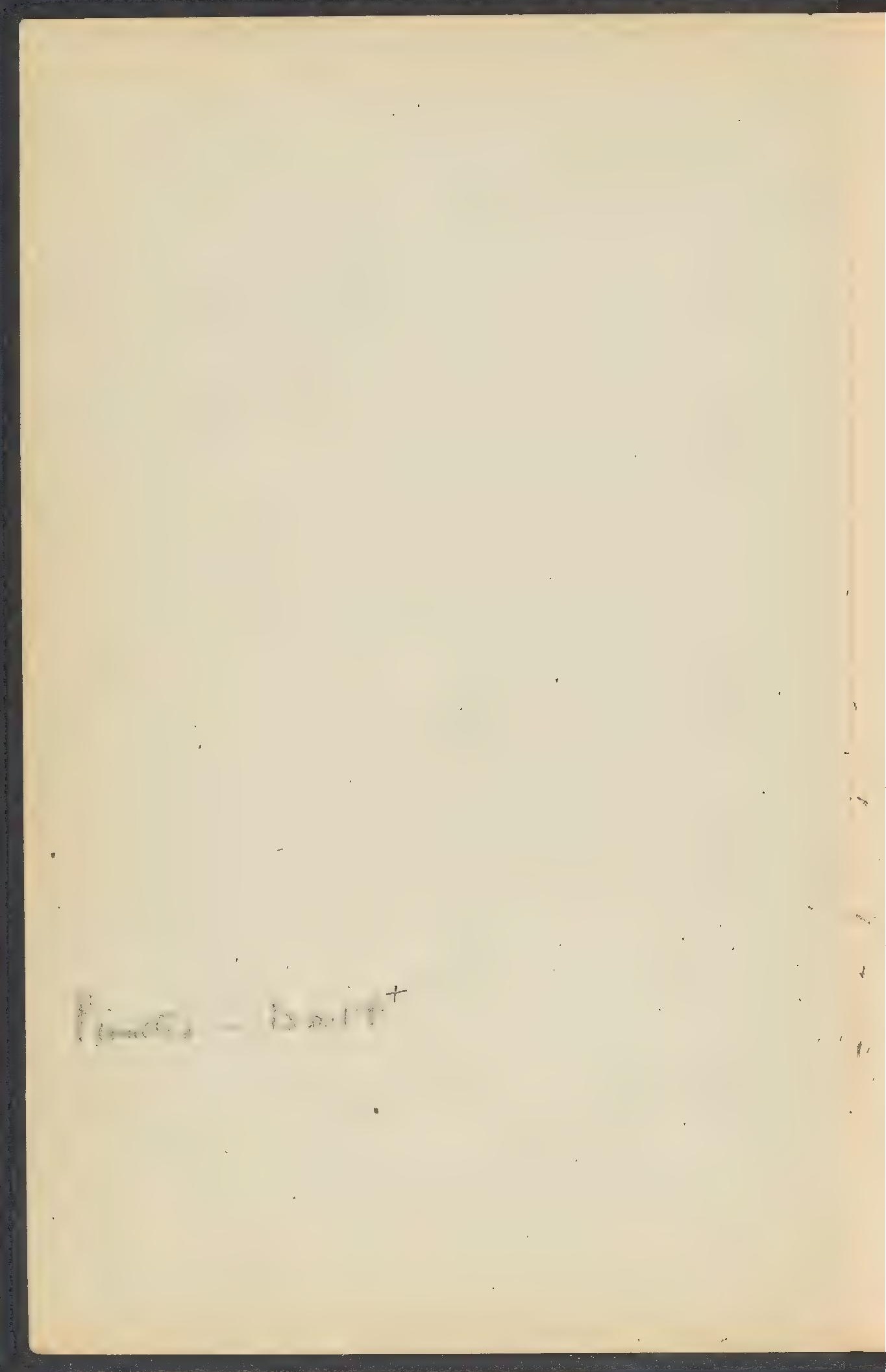


hedeoma, Pers. American Pennyroyal.

H. pulegioides $\left(\mathrm{L}_{\text {. }}\right.$ ), Pers. American Penmyrotal.

Common in dry soil.

\section{NEPETA, L. CATNIP.}

y 21 N. Cataria, L. Catmip.

Rare. Roadside near $0^{\prime}$ Connor Cove; Thompson Island $\mathbb{S}_{n}$ (Annie S. Downs); - waste ground near dwellings, Fernald Cnip Point; Sutton Island (Rand). Adventive from Europe. $\quad$ a $/$.

Y 22 N. Glechoma, Benth. Ground Ivx. Grlmover-the-groum.

Door yards and waste grounds; infrequent. Somesville; Southwest Harbor (Rand). Adrentive from Europe. of Caul $4.7 \mathrm{C}$

\section{SCUTELLARIA, L. SKULlCAP.}

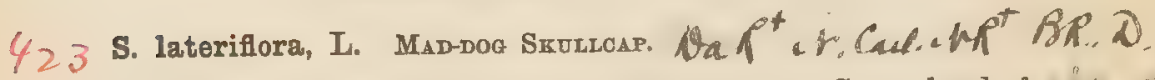

Wet shady places; infrequent. Marsh, Valley Cove; head of Northeast Creek; High Head meadow (Rand); - Somesville (Annie S. Downs, Raud); - "Mt. Desert" (F. M. Day); - Bar Harbor (W. H. Manning). qc

S. galericulata, L. Common Skdlicap. Q C

Common on sea beaches and banks by the shore; more rarely on pond shores. Cuf. XC. $B$.

Forma rosea.

Flowers rose-color. Beach, Great Cranberry Isle (Rand).

BRUNELILA, L. Self-heat.

B. vulgaris, L. Coмmon Self-hear.

Woods, fields, and roadsides; common.

Forma albillora. $\quad G_{N}$, Bie cr. Car. KC.

Flowers pure white. Meadows above Long Pond (Redfield). 


\section{LEONURUS, L. MOTHERWORT.}

I. Cardiaca, L. Common Motherwort.

Waste places and by dwellings; rare. Tarr Valley, near Fer- $Q_{s}<$. nald Point (Annie S. Downs); - Oak Hill; Sargent Cove (Rand). Adventive from Europe.

\section{GALEOPSIS, L. HeMP NETTLE.}

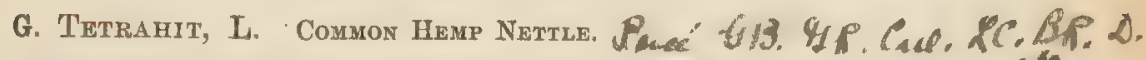
A common weed in waste places and in cultivated grounds. Said to be naturalized from Europe, but appearing indigenous, at least in northern New England.

STACHYS, L. Hedge Nettle.

S. ARVENSIS, L. WOUNDWORT.

Rare. Field, Bar Harbor (Mary Minot). Adventive from Europe.

\section{Pliantaginace A. Prantain Familu.}

\section{Plantago, L. Plantain.}

\section{P. major, I. Commox Plantaix. $4 \mathcal{K}^{+}$Bie}

Fields and waysides; very commoh. Commonly naturalized from Europe. This plant, however, is found all over the Island in many places so remote from dwellings or cultivated grounds as to lead to the inference that it may be also indigenous. Thick-leaved saline forms are frequent on sea beaches. Little Harbor; Little Cranberry Isle (Redfield); - Great Crauberry

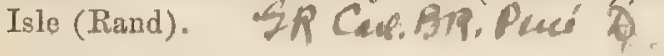

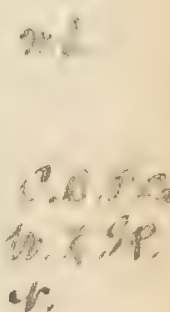

P. laxceolata, L. Ribgrass.

Waysides and grass lands; formerly rare, but of late years $F \cdot V_{r}, t$ becoming more common. Mill Cove; Little Harbor; Southwest Harbor (Rand); - Bar Harbor (Rand, W. H. Manning).

P. decipiens, Barn. Sea Plantain, mi $T$

Very common in salt marshes, on beaches, and among rocks on the shore. Carf. BR. PR. AxR. 
P. Patagonica, Jacq., var. aristata (Mx.), Gray.

Rare. Field, Southwest Harbor (Annie S. Downs); - Bar Harbor (W. H. Manning). Adventive from the West.

Division III. APETAL

\section{AMARANTACEAE. Amaranth Family.}

AMARANTUS, I. Amaranth.

A. retroflexus, L. Amarante Pighegd.

Roadsides and cultivated grounds; becoming frequent. Bar fo Harbor; Southwest Harbor; Somesville; Long Pond, etc. (Rand); - Seal Harbor, etc. (Redfield). Adventive from Tropical America.

A. albus, L. Tumbleweed.

Cultivated grounds; becoming frequent. Southwest Harbor; Long Pond; Seal Harbor, etc. (Rand); - field on Northeast Creek (M. L. Fernald). Adventive from the South and West.

\section{CHENopodiace $\bar{E}$. Goosefoot Family.}

\section{SPINACIA, L. SPINACH.}

Diœcious; flowers axillary, glomerate. Staminate flowers in racemose-paniculate clusters, calyx 4-5-parted, the lobes equal. Calyx of pistillate flowers ventricose-tubular, 2-3-toothed; ovary ovoid, styles 4, elongated, filiform, achene included in the turgid indurated calyx, which is often 2-3-horned on the back; seed vertical and compressed; embryo annular, surrounding the farinaceous albumen. Darlington, Am. Weeds and Useful Plants (Rev. ed.), 274.

S. GLABRA, Mill.

Herbaceous, glabrous throughout; leaves sagittate, sometimes oblong-ovate, entire, acute, slender-petioled; flowers green; fruiting calyx solitary, rounded, without prickles, toothed at 
the apex. [For a more detailed description, see DC. Prod, xiii. 2. 118.] Abundant in waste ground, Somesville (Rand). Adventive from Asia.

CAENOPODIUM, L. Pigweed.

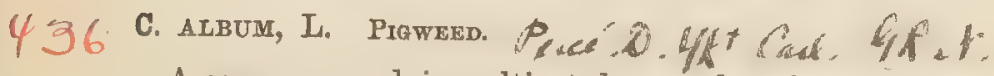

A common weed in cultivated ground, and on sea beaches. $C$. Very variable. Naturalized from Europe. T

\section{ATRIPLEX, L. ORACHE.}

A. patulum, L., var. hastatum (L.), Gray. A. Aft- Ade. AR. Bic Sea beaches, salt marshes, etc.; very common and variable. Both prostrate and erect forms are found. T Var. littorale (L.), Gray.

Rare. Beach, Great Cranberry Isle (Rand). ers $_{4}$.

\section{SALICORNIA, L. SAMPHIRE.}

S. herbacea, I.

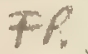

Salt meadows, and muddy shores on the coast; common.

SU IEDA, Forskal. SEA Blite.

S. 'linearis (E1l.), Moq.

Common on sea beaches.

SALSOLA, I. SALTWORT.

Sea beaches; frequent, especially on Cranberry Isies. The Cent

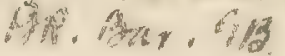

POLYGonaCEE. Buckwheat Famili.

RUMEX, L. Dock. SORrel.

R. Patientia, L. Patience Dock.

Rare. Dry fields near Little Harbor (Redfield). Adventive from Europe. 


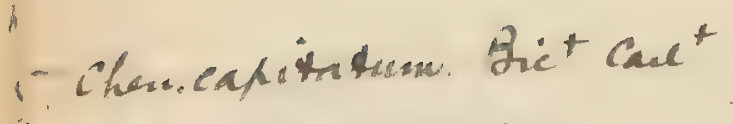

chen. murale tht

chen. Ifuluari lyt

$\therefore \bar{r} \cdot 1 k^{+}$

atr. Perei

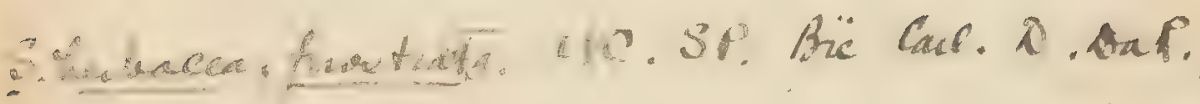

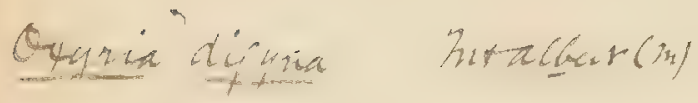




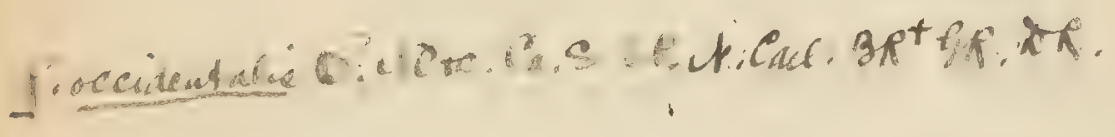

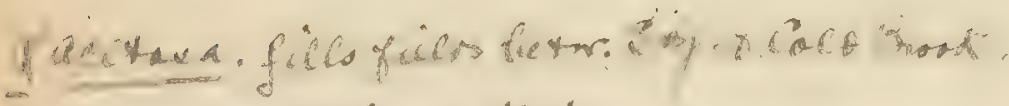

T. Encephacophoine $\mathrm{f} / \mathrm{e}^{*}$

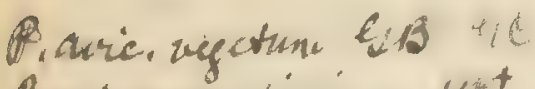

Piavie anguntissimuma $1 / t^{+}$

B rowiticums.. W.

$\rho-m-j$

Poxsertum B 


\section{R. Britannica, L. Great Water Dock.}

Common in swamps and wet places back of sea beaches; less $C, P$. common in wet ground farther inland, - Long Pond meadows; $\mathrm{SC}^{+}$ Somesville (Redfield). fif. CALP. B

R. salicifolius, Weinm. Wuтre Dock. Aaf. M

Sea beaches; frequent, especially on Cranberry Isles and $C$ a $R$ western and northern shores of the Island. Also Northeast Harbor; Sea Wall (Rand); - Bar Harbor (W. H. Manning). B W ' R. verticillatus, I. Swamp Dook.

Rare. Marsh on Northeast Creek; Norwood Cove (Rand).

R. CRispus, L. Curhed Doox.

Common in cultivated and waste ground. Naturalized from C.P.S,

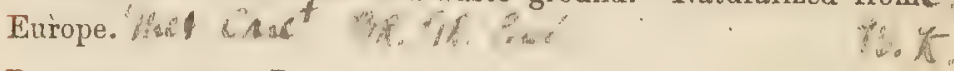

R. OBtusifotius, L. Bitter Dock.

Waste places; rare. Somesville (Rand); - Bar Harbor (W. H. Manning). Adventive from Europe. Thet. Beple Pece $\mathrm{yt}^{t}$

R. Acetoselida, L. Freud Sorrer. /hd, T

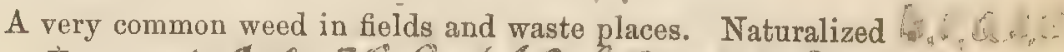

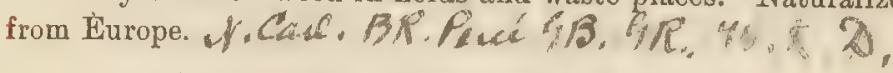

POLYGONUM, L. KNOTWEED.

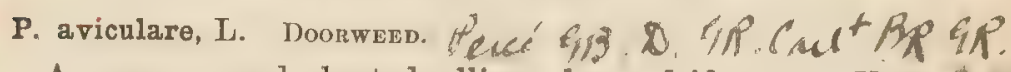

A common weed about dwellings, by roadsides, etc. Variable; erect or prostrate.

P. Raii, Bale. $T /, C_{2 i}$,

U.

Stems long, straggling, prostrate; leaves bending towards $C$ the stem, elliptic-lanceolate, flat; ochrex lanceolate, acute, with few distinct simple veins, at length torn; nut smooth, shining, exceeding the perianth. Resembling $P$. aviculare in habit, but $P$. maritimum in fruit. Filaments broader at the base. It varies with smaller leaves and flowers. Babington, Man. Brit. Bot. (4th ed.) 285. Common on sea beaches. This species has been often mistaken for $P$. maritimum, and so reported. So far as known, however, $P$. muritimum is not found within the 
limits of this list. The specimen attributed to Mt. Desert in Bull. Torr. Bot. Club, xix. 362, is now pronounced to be $P$. Raii (fide J. K. Small).

P. lapathifolium, L.

In cultivated grounds; rare. Southwest Harbor (Rand). Doubtless introduced.

P. Hartwrightii, Gray.

Rare. Bog, Southwest Harbor (Rand). $6 . \&, i_{i} p^{+}$

P. Careyi, Olney.

Rare. Wet ground on wood road, Town Hill (M. L. Fernald).

P. Persicaria, L. Ladx's Thumb.

Common in waste and damp places. Variable. Naturalized from Europe. Bre. BA. Peses \&, A, \&B, T it. of

P. Hydropiper, L。 WATER Pepper.

Common in wet places.

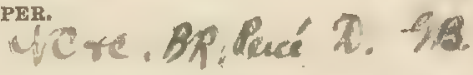

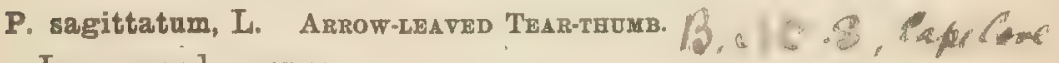
Low grounds; common.

P. Convolfulus, L. Black Bindweed.

Waste grounds and beaches; common. Naturalized from B.PCA.

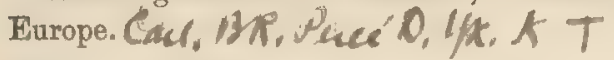

P. cilinode, $\mathrm{Mx}$. qe nu.

Woods and copses, especially in clearings; common. $C$. A. O Y Cein

FAGOPYRUM, Gritn. BuCKWHEAT.

F. enculentum, Moench: Peed D T

Waste ground, Southwest Harbor (Rand). Adventive from : d w Europe.

EUPHORBIACE AE.' SPURge FAMmY.

EUPHORBIA, L. SPURGE.

E. Ctpartisstas, L. Gravetard Flower.

Escaped from cultivation to roadsides and waste places. Bar Harbor; Southwest Harbor; High Head (Rand); - Town Hill (Greenleaf, Lane \& Rand); - Somesville (R. \& R.). Naturalized from Europe. 


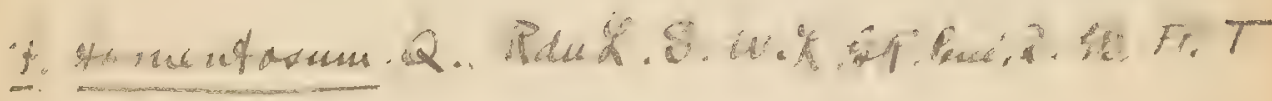

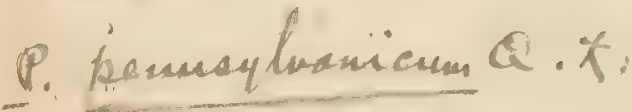

Paruphitium byt

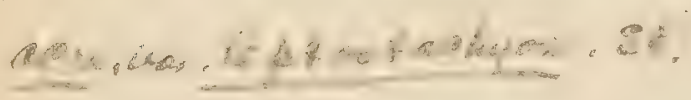

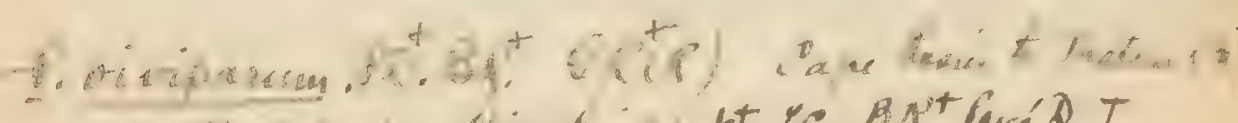

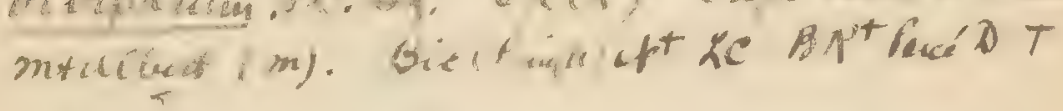

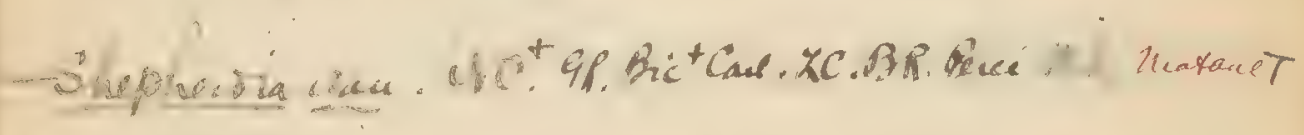

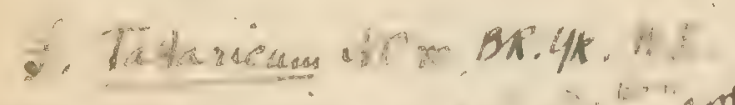

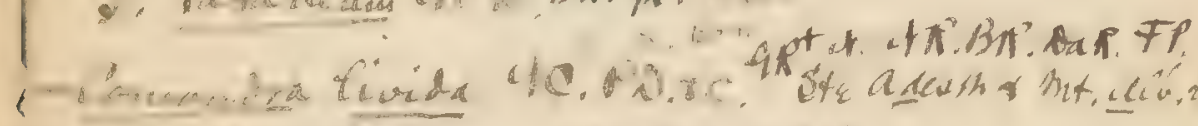

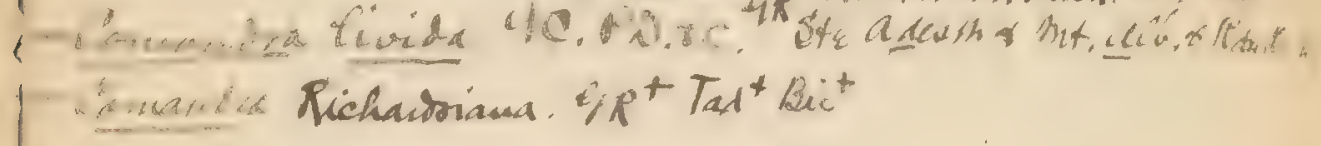

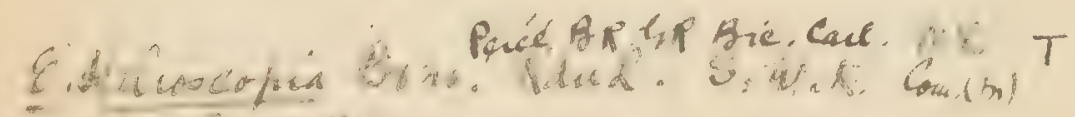

E. Grisin, $\%$,

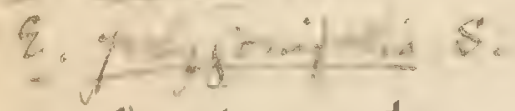

E. hiresta mext, 


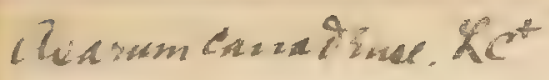

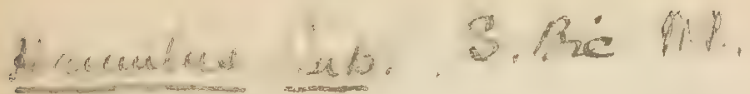

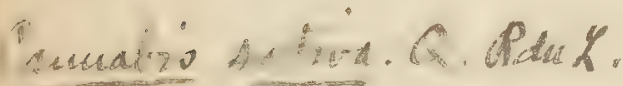

$11 \cdot \operatorname{tin} e^{2} \cdot 4 \cdot 4 k^{t}$

valponta 8., thet. 1:Caul, te

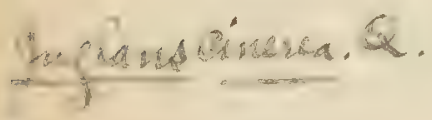




\section{URTICACE F. NeTtle FAMilu.}

ULMUS, L. EnM.

\section{$46 /$ ๘. Americana, L. American Elm.}

Rare. Fields and roadsides, Eden (Rand). Introduced in $C_{\text {. }}$

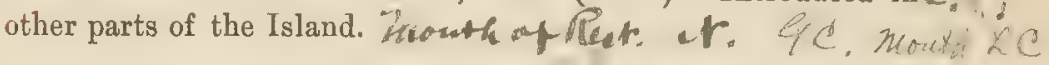

\section{URTICA, L. Netthe.}

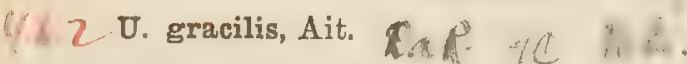

Frequent about and at the heads of sea beaches. Sometimes $C_{0}+A_{0}$ in waste ground. thet, Pr. Die. Cal, Pesé

* U. URENs, L.

Rare. Shore of Little Cranberry Isle (Redfield). Adventive $B_{0}$, from Europe. AR+ $9 R$.

\section{PARIETARIA, L. Pelltory.}

P. Pennsylvanica, Muhl.

Rare. Shores of Little Cranberry Isle (Redfield). Doubtless naturalized from farther south.

\section{MYRICACE E. Sweet Gate Family.}

\section{Qle t d MYRICA, L. BAFBERRT.}

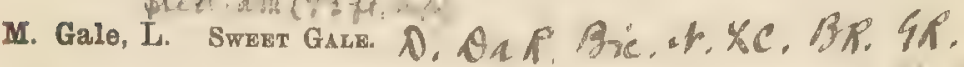
Borders of ponds, streams, and wet meadows; common. $C .6$. Ala 4.8. M. cerifera, I. BAYBERRY.

Rocks on coast; common. Also Browns Mt.; Somesville; $\$ . f^{-}$ Mt. Desert Narrows, etc. (Rand).

M. asplenifolia, L. Comptonia asplenifolia (L.), Ait. SweEt . FERN.

Dry hills, fields, and borders of woods; common.

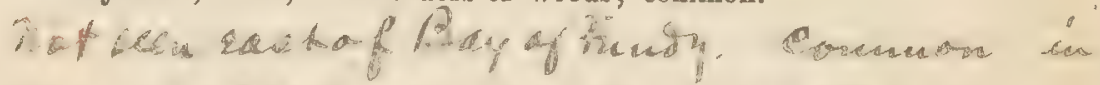

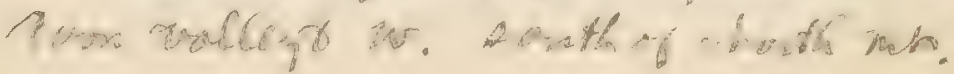




\section{CUPUlifer $\mathbb{E}$. Oak Family.}

BETULA, L. BIRCH.

B. lenta, L. Black Birch. Sweet Birch.

Woods and copses; frequent.

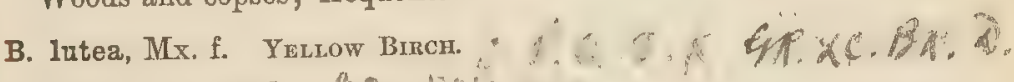
Common in woods. $9 \mathrm{C}$. NAt Re

B. populifolia, Marsh. White Birch. Grax Btrch. C. † Very common in poor soil.

B. papyrifera, Marsh. B. papyraced, Ait. PaPer Bircr. Canoe

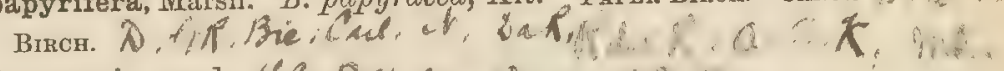

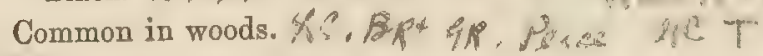
Rega -

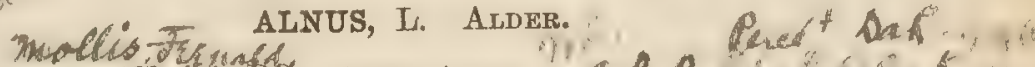

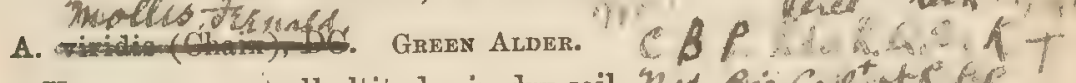

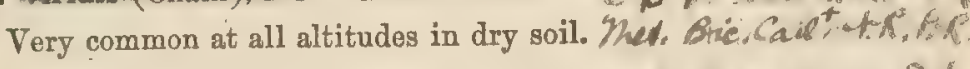

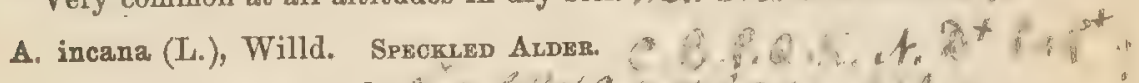
Common in low grounds. $>4$.

\section{CORYLUS, L. HAZEL-NUT.}

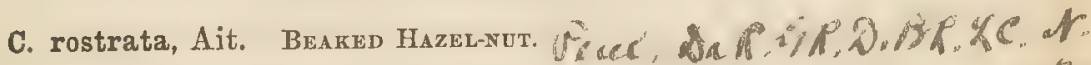
Frequent in woods and clearings, and by roadsides. Northwest Cove; Emery District; Dog Mt.; Sargent Mt.; Aunt Mollys Beach; Somesville, etc. (Rand);-Echo Notch (R. y Cad. \& R.). GC

\section{QUERCUS, L. OAK.}

Q. rubra, L. RED $O_{\Delta K}$.

Frequent; widely distributed, but nowhere very abundant; rare in the south of the Island. Much dwarfed on the mountains. During the early bistory of the Island oaks were apparently abundant, although probably of this species only. At a very early day, however, the oak woods began to be felled for timber. At Somesville, it is said, there was an oak wood on the shores of Somes Harbor, which attracted the attention of 


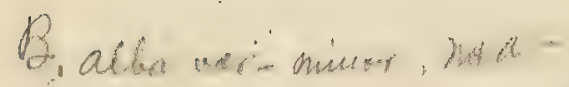

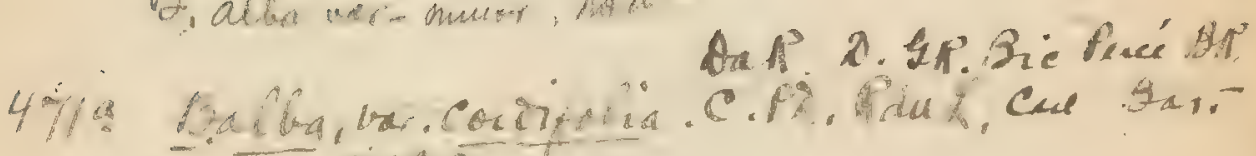

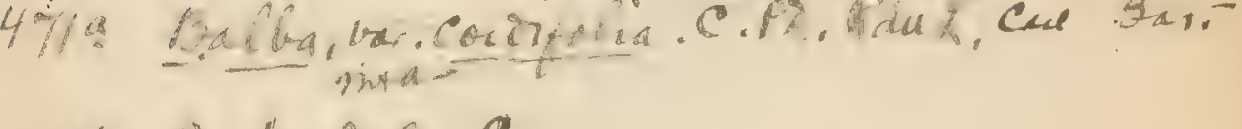
47 b. 13. prumbera C.

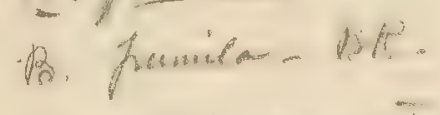

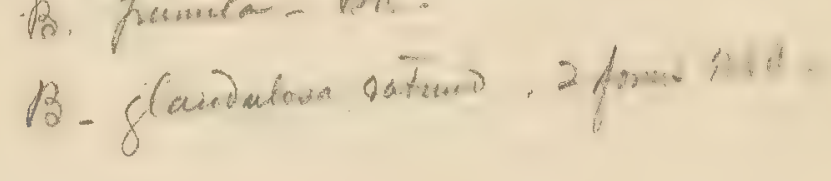


Q S int 1

2 niras.

$\therefore \quad-\infty \quad: 10$.

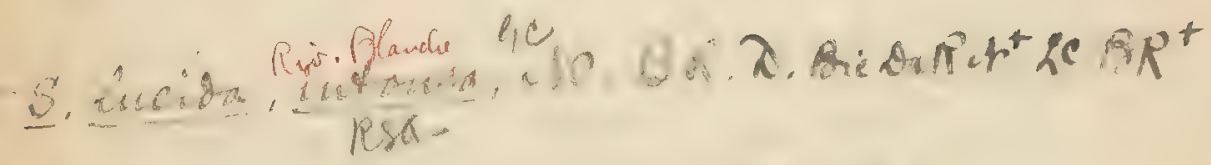

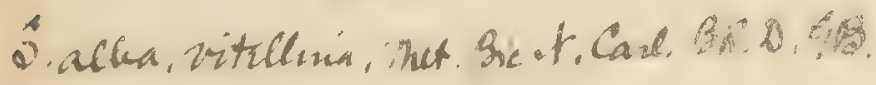

$2 \operatorname{ciscos}$

5 mint 
Abraham Somes, of Gloucester, Mass., when he chanced to sail up the Sound about 1760. He spent the summer in that vicinity making barrel staves, and then returned home with his cargo. The following year he returned to Somes Harbor for the same purpose, and finally, in 1762, built a house on the shore of the harbor near the present steamboat wharf, and began the permanent settlement of Mt. Desert Island.

Y/7 Q. ilicifolia, Wang. Bear OAK. BuACK Screb OAK.

Rare. Dog Mt. (Rand, Elizabeth G. Britton).

FAGUS, L. BEECH.

F. ferruginea, Ait. American Beech.

Woods; common.

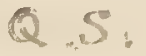

SALICACE $\bar{A}$. Willow Family.

SALIX, L. WILLOW.

S. lucida, Muhl. Strinrrg Wrulow.

Wet places and borders of brooks and ponds; frequent. Otter $C$ Creek Brook; Great Pond; Northwest Cove, etc. (Rand); $-Q .1$ at Bubble Pond; Jordan Pond; Long Pond meadows, etc. (Red-

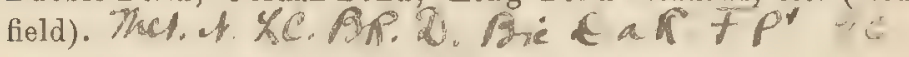
Forma latifolia.

Leaves $1_{\frac{1}{2}}^{\prime}$ wide, rounded or subacute at base; cuspidateacuminate. Swamp north of Beech Hill; Southwest Harbor (Rand); - Bubble. Pond (R. \& R.).

Forma angustifolia. $B p^{+} D$. OR $R^{+} \cdot R \& A$

Leaves narrowly lanceolate, tapering to a long point. Long Pond meadows; Thompson Island, etc. (Rand).

S. Fragilis, Ld Crack Willow. Brittie Willow.

Bog, Clark Point, Southwest Harbor; Somesville (Rand). Naturalized from Europe.

S. FRAGILIS $\times$ ALBA, Wimmer.

Aments leafy-peduncled, slender, loosely flowered; stamens 2, villous at base; scale yellowish, lingulate; capsule very shortpedicelled, conico-cylindrical, glabrous; style very short, stig- 
mas spreading, recurved, 2-lobed; gland embracing the pedicel; leaves broadly lanceolate tapering to a prolonged slender point, glabrous and shining above, silvery-silky beneath when young. Wimmer, Salices Europææ, 133. Southwest Harbor (R. \& R.).

All the common large willow trees of the Island probably belong either to S. fragilis or to this hybrid. Both were of early introduction, and have become spontaneous all over the Island. Forms of $S$. alba may also be looked for.

S. rostrata, Richardson. S. livida, Wahl., var. occidentalis,

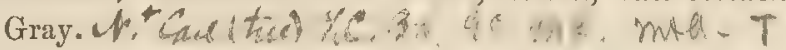

Very common in either wet or dry soil.

s. discolor, Muhl. "Common Swamp Willow. $C$

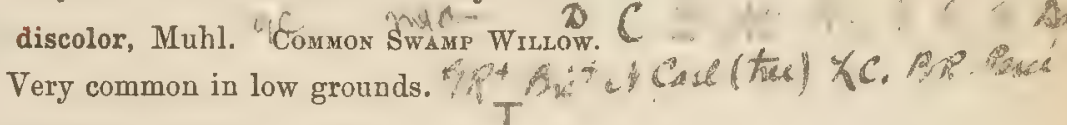

\section{S. humilis $x$ discolor, Bebb.}

Leaves as broad and large as those of $S$. humilis, but duller green, softly tomentose beneath, and with shorter petioles; the aments thick as those of $S$. humiiis, but usually recurved, and the capsules on shorter pedicels. Bebb, Gray Man., 6th ed., 483. Swamp, Southwest Harbor (Redfield).

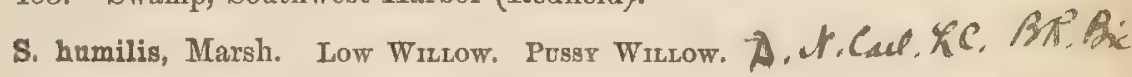

Common in dry or wet ground everywhere from sea level to mountain summits. Forms with large leaves may be described as follows:- 40 $m a$, ma $-T$

Var. grandifolia, Anders. DC. Prod., xvi. 2. 236.

Leaves obovate-oblong $3^{\prime}-4^{\prime}$ long, $1 \frac{1^{\prime}}{2}$ broad above the middle, shining above. Appearing in the following forms:-

\section{Forma obtusifolia.}

Leaves very short-pointed; either smooth and glaucous, or slightly tomentose beneath. Near Great Pond (R. \& R.); Sutton Island (Rand).

\section{Forma acuminata.}

Leaves more attenuate-pointed; glaucous and tomentose beneath, generally becoming smooth. Seal Harbor (Redfield); wood road to Denning Pond (Rand). 
S. ampíân. $m i n t$

b. roctica medebar(in)

S. wat, ma.? mot ${ }^{4}$

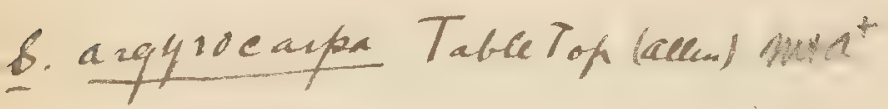

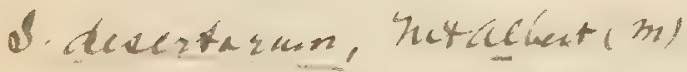

Sidever unicta, mat

"S.tucoutepu wh"

Q ataces rut. mitat

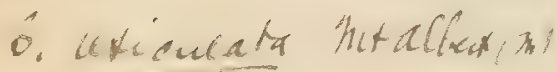

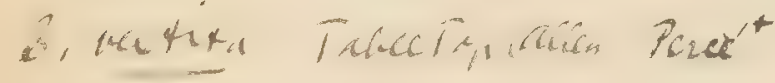

S. phlicifolia $m+a$ 
S. manciat? th

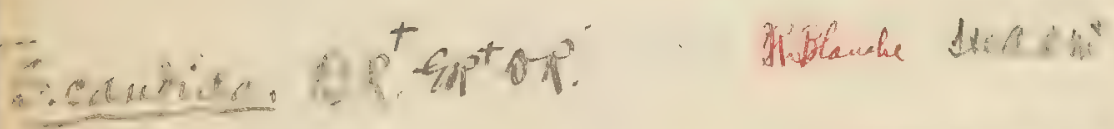

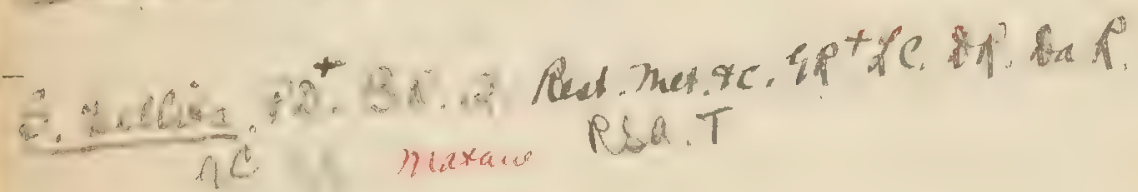

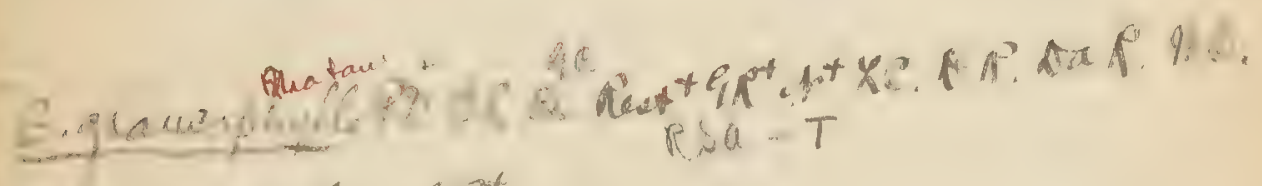

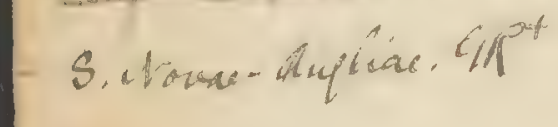

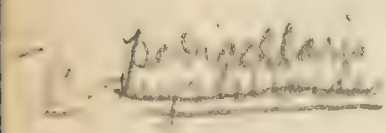

nulutiont ant

S. herbeca. motriat

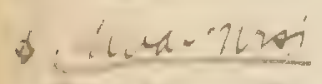


This species and others, especially $S$. discolor, often bear coneshaped galls on the ends of the branches. These consist of imbricated leaves, and are caused by the deposit of eggs of insects.

\section{S. tristis, Ait. DWARF GRAY WiLlow.}

Rare. Hollows, between Northeast Harbor and Little Harbor (Rand). Perhaps introduced in this locality from beyond our limits.

\section{S. petiolaris, Smith.}

Frequent. Somesville; Bass Harbor Marsh; Great Cran- C. berry Isle; Long Pond meadows, etc. (Rand). The type passes into the next variety.

Var. angustifolia, Anders. W. rosmarinifolia (Herb: Hook.), Barratt \& Hooker.

Leaves narrowly lanceolate, almost linear, margin slightly serrulate or subentire, glancous beneath, at first silky, at length glabrate. DC. Prod, xvi. 2. 234. Common on meadows and heaths. Long Pond meadows; Pond Heath; Northeast Meadow, etc. (R. \& R.). The silky hairs of the young leaves are usually of a rusty color.

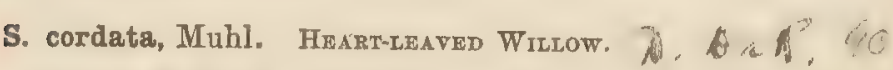

Rare. Roadside near Denning Pond; Southwest Harbor 6 ,

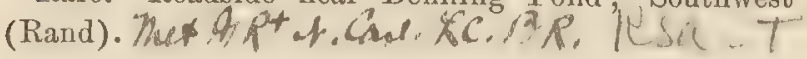

S. balsamifera (Hook.), Barratt. Batsam WiLLow.

Widely distributed, but nowhere very abundant. Sonthwest Harbor; Sargent Mt.; Beech Hill; Ripples Pond; Somes Pond; Denning Brook; Beech Mt. Notch; The Hio; Doctors Brook; road, west side of Browns Mt., etc. (Rand); - Seal Harbor Ca (Redfield); - Browns Mt. (E. Faxon); - High Head meadow (Faxon \& Rand); - Long Pond meadows ( $R_{0} \& R_{4}$ ).

POPULUS, I. POPLAE Aspren.

P. tremuloides, Mx. Aspen. "D. B Frequent in woods.

$$
\text { if } f_{1}, \mathbb{R} \text {. }
$$


P. grandidentata, Mx. LARGe-Toothed Aspen.

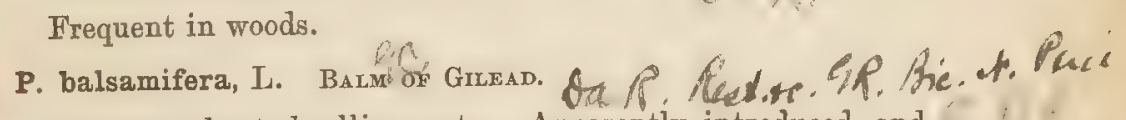

Frequent about dwellings, etc. Apparently introduced, and

RGQ T naturalized by seedlings. Oak Hill, etc. (Rand); - Southwest Harbor (Elizabeth G. Britton, Rand); - Seal Harbor (Redfield); - and elsewhere. At Somesville, however, and east of Town Hill, remote from dwellings, it appears to be indigenous.

P. dilatata, L. Lombardx Porlar.

Occasionally by roadsides, etc., and sometimes spontaneous, $\sqrt{8}$. Somesville; Southwest Harbor (Rand); - Oak Hill (Redfield). Adventive from Europe.

\section{EMPETRACE AE. Crow berry Family.}

EMPETRUM, L. CROWBERRY.

499 E. nigrum, L. Buack Crowberry. R Rlancle

Common on cliffs along the coast and on the islands. Often in heaths and bogs; The Heath, Great Cranberry Isle; Sunken Heath; Great Heath (Rand); - more rarely on mountains and hills; Barr Hill; Green Mt. (Redfield); - Sargent Mt. (Rand). Also Duck Islands (Redfield).

\section{COREMA, Don. Broom Crowberry.}

C. Conradii, Torr.

Dry rocky places; rare and local. Asticou; Little Harbor (Lane \& Rand); - Barr Hill, in a number of localities; hill between Long Pond and Little Harbor Brook (Redfield); Beech Mt.; Dog Mt. (Rand); - Beech Hill (Annie S. Downs, Sara E. Boggs); - Ship Harbor (Annie S. Downs); - in great abundance in pine barreus, east of Ship Harbor; west of Hio (Rand).*

* Corema also grows in abundance with Pinus Banksiana, near Prospect Harbor, Gouldsborough (Redfield). 


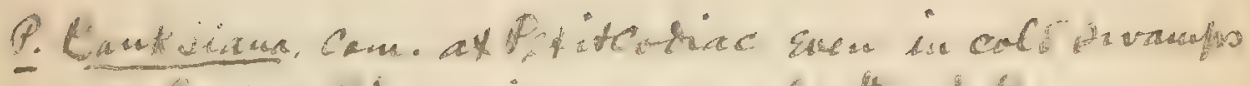

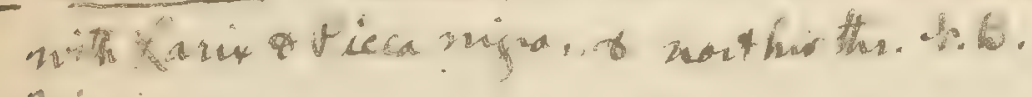
Bre. 


\section{Class II. DICOTYLEDONES GYMNOSPERME}

CONIFERAE. Pine Family.

PINUS, L. PINE. *

\section{P. rigida, Mill. Pтtch Pars.}

P. Strobus, L. Whixw PLNe. Rsapeaces

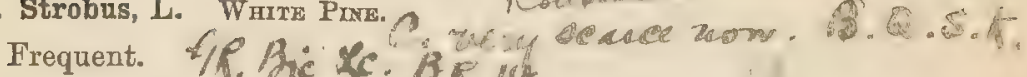

Barren soil; not uncommon, but local. Browns Mt., etc. (R. \& R.). Very abundant on Newport Mt.

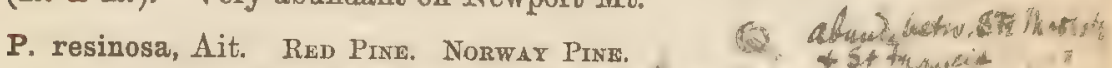

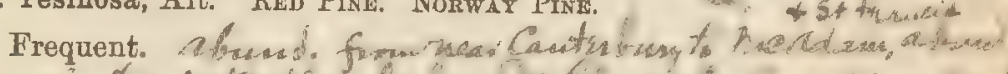

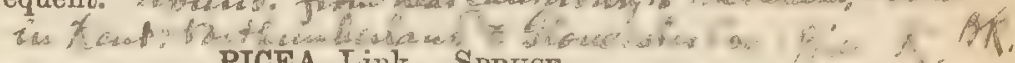
PICEA, Link. SPRUCE.

P. nigra (Ait.), Link. Abies nigra, Poir. Black Sprece.e.

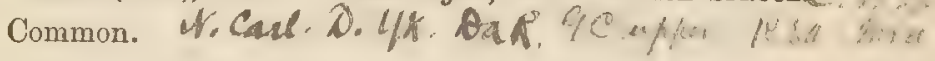

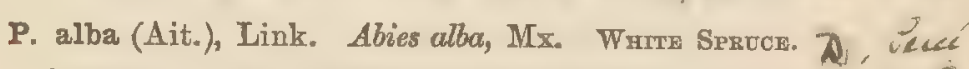

Common. More common near the coast than the preceding $C \&$ species. A very beautiful glaucous or blue form is not uncom- if

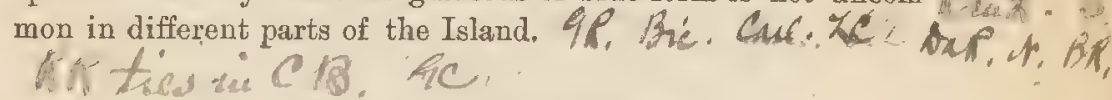

TSUGA, Carr. HemLock.

5. 7 T. Canadensis (L.), Carr.

Infrequent, except in old woods. Also on Cranberry Isles. $f$.

ABIES, Juss. FIR.

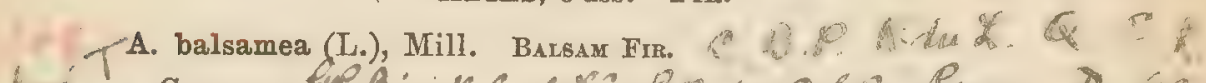

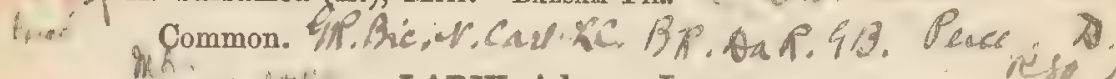
Wh. hathe tarix, Adans. LARCH.

Tof fimithe LARIX, Adans. LARCH.

I. Americana, Mx. Hackmatagk. Tamarack.

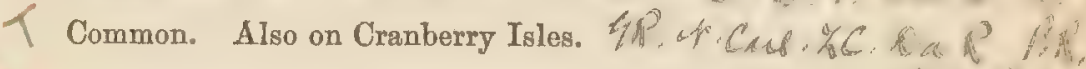

* P. Banksiana, Lamb., is found in abundance on Schoodic Peninsula, across Frenchman Bay, but has not yet been found on Mount Desert Island. 
THUJA, L。ARBOR-VIT雨。

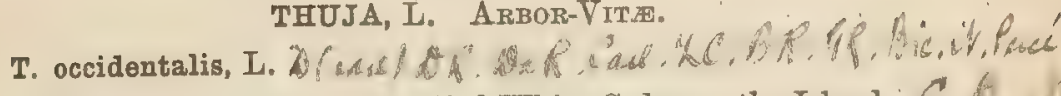
Very common. Usually called White Cedar on the Island. etraw $R \&$. JUNIPERUS, L. JUNIPER.

J. communis, L. Coмmon Juniper.

Common in dry ground, on rocky cliffs, etc. Very low-spreading, and variable. War. Hie. Cond. Pewe

J. Sabina, L., var. procumbens, Pursh. Cresprang Juniper.

Frequent in dry. fields or on rocky eliffs along the coast. Roberts Point, Northeast Harbor; Pierce Head; Sargent Mt. C. Bref (Rand); - shores Seal Harbor (Redfield); - Bar Harbor (Beacerl, Pscé trix Jones); - Bald Porcupine Island (W. H. Manning); very common in dry fields, Sutton Island (Rand et als.).

T. Canadensis, Willd. Yew. Ground Hemlock.

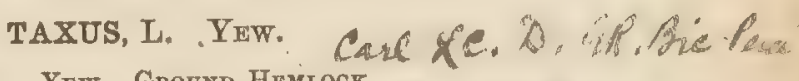
Common in deep moist woods and glens. की कि

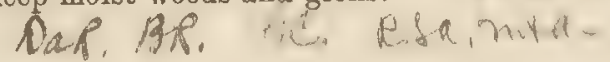

\section{Class III. MONOCOTYLEDONES.}

\section{ORCHIDACEA. ORChid FamilY.}

\section{MICROSTYLIS, Nutt. ADDER's MoUtH.}

5.14 M. ophioglossoides (Willd.), Nutt. Rif /n. Peat Bic

Not infrequent, and very generally distributed over the Island and the Cranberry Isles, in low moist ground. Also Sargent

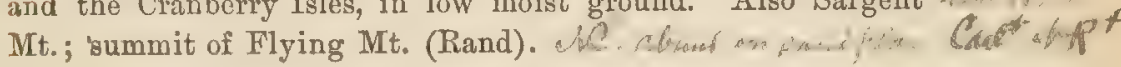

LIPARIS, Richard. TWaYblade.

L. Looselii (L.), Richard.

Local. On Stanley Brook, Seal Harbor (G. Hunt); - swamp on Breakneck road (Brigham); - near Kings Point, and on

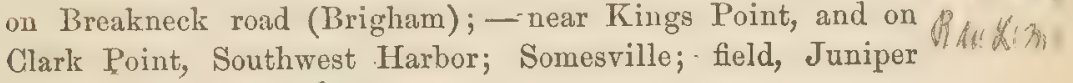
Cove (Rand). $\therefore$. PR. 


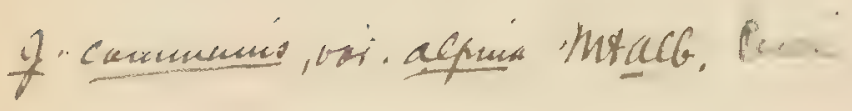

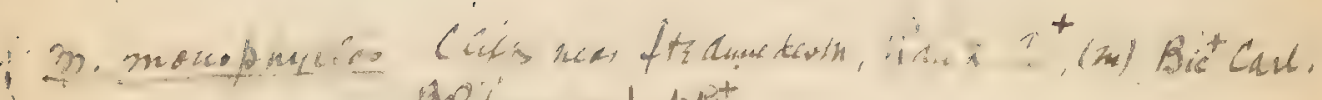

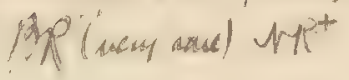

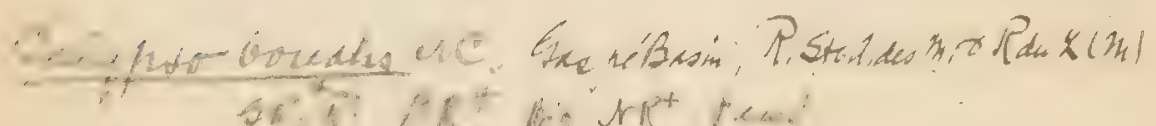

i 
b. : $: 4, \quad+\cdots$

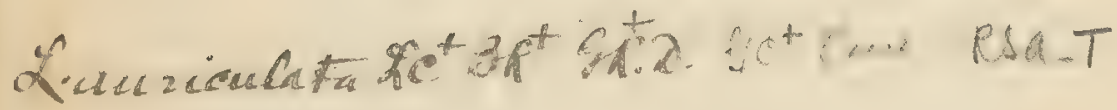


CORALLORHIZA, R. Br. CORAL-ROOT.

C. innata, R. Br. 2. GA R C.AP.T

Deep woods; infrequent. Hadlock Upper Pond (H. C. Jones, Rand); - Southwest Valley; Cold Brook; Little Harbor Brook Notch; Clark Valley (Rand); - Sargent Mt. (Brigham); woods, Jordan Pond road (Redfield); - woods, off Town Hill F/P, Cue, ct,

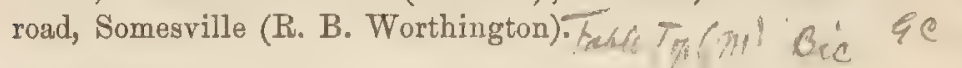
C. multiflora, Nutt.

Infrequent, and not as widely distributed as the last. Hadlock Valley (Rand, Redfield); - Bar Island, Bar Harbor (F. M. Day); - woods, Hadlock Upper Pond; Cold Brook; Clark Valley (Rand). Pas \&in! thet.

LISTERA, R. Br. TWAYBlade.

L. cordata (L.), R. Br. Ruce KC. Q Y

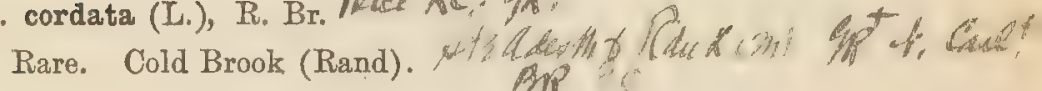

L. convallarioides (Swz.), Nutt.

Very rare. Woods, head of The Barcelona meadow (Rand).

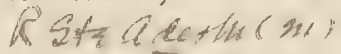
Mr? $1<$ SPIRANTHES, Richard. LAdieis Tresses.

\section{S. Romanzoffiana, Cham.}

Frequent in damp ground or meadows. Flowering in late

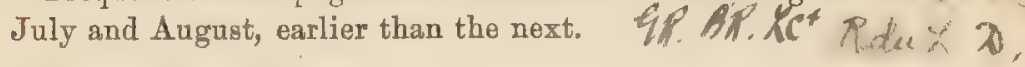

S. cernua (L.), Richard.

Very common in damp ground. Flowering in late August, $s$. September, and early October.

S. gracilis (Bigel.), Beck.

Infrequent. Roadside near Little Harbor (W. C. Lane); Beech Cliff; roadsides, Northeast Harbor, and near Fernald Cove; Norwood Road, Southwest Harbor (Rand); - Barr Hill; fields north of Long Pond (Redfield); - Southwest Harbor (Annie S. Downs). 


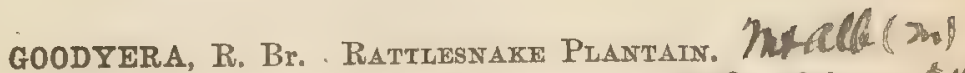

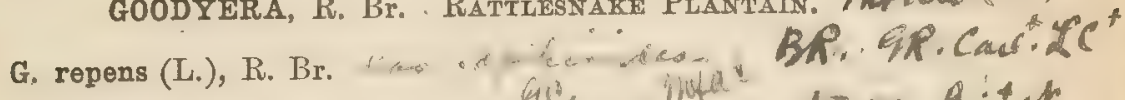

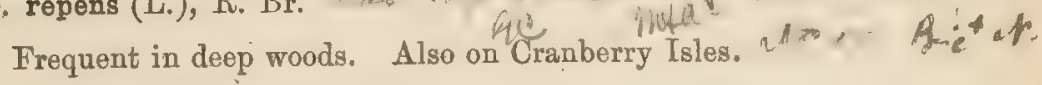

G. pubescens (Willd.), R. Br.

Rare. Sargent Mt. Gorge; Cold Brook (Rand).

ARETHUSA, L.

A. bulbosá, L.

Common in sphagnum bogs. A form with two scapes, each two-flowered, Sea Wall Swamp; meadow on Denning Brook (Rand).

Forma albiflora.

Flowers pure white. Sea Wall Swamp (Redfield, Faxon \& Rand); - Somes Pond Swamp; Sunken Heath (Rand); - Great Heath, etc. (Annie S. Downs).

Forma subcærulea.

Flowers bluish or lavender in color. Swamp on Denning Brook (Rand).

CALOPOGON, R. Br.

C. pulchellus (Willd.), R. Br.

Common, usually in sphagnum bogs.

POGONIA, Juss.

P. ophioglossoides (L.), Ker.

Common in sphagnum bogs and on wet pond shores. On the Island this and the two plants last named are called indiscriminately by the name of Swamp Pink.

Forma albitlora.

Flowers pure white. Jordan Pond (Rand); - Sea Wall; Great Heath (Annie S. Downs). 


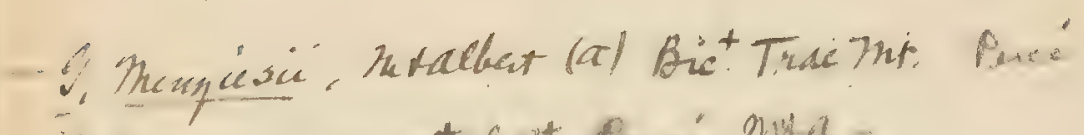

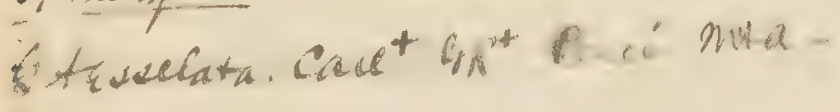




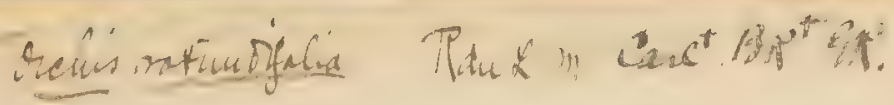

4. Gencerata ripuximi

1 Lacera. S. $x$

$$
k^{2}-3 .
$$

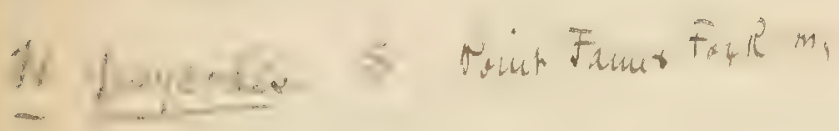

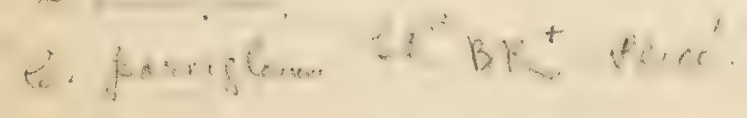


HABENARIA, Willd.

H. tridentata (Willd.), Hook.

Common in moist ground.

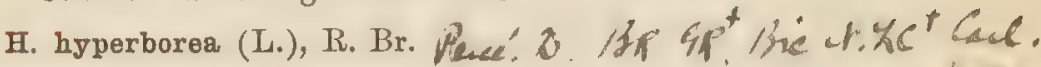

Rare. Swamp, Northeast Harbor (Rand); - trail between Jordan Pond and Northeast Harbor (Redfield). $R$ \& $C$, 7

H. dilatata (Pursh), Gray.*

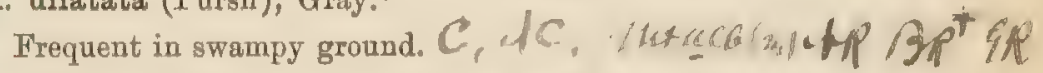
H. obtusata (I'ursh), Richardson. 2. Fi, Cul th. In th ic

Deep, mossy woods; infrequent. Sargent Mt. Gorge; woods, head of The Barcelona meadow; Cold Brook (Rand); - Seal Harbor (Redfield); - Little Cranberry Isle (Redfield, Harriet $\lambda$

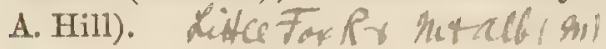

H. Hookeri, Torr. †

Rare. Woods near Otter Creek (Helen B. Walley). Rid H. orbiculata (Pursh), Torr.

Deep woods; infrequent. Sargent Mt. Gorge; Northwest. St ? by \% Arm woods (Rand); - woods, southern end of Great Pond (An- $/ C$ nie S. Downs); - Seal Harbor (Redfield, Lizzie Churchill); - Little Cranberry Isle (Redfield, Harriet A. Hill).

H. fimbriata (Ait.), R. Br. Purple-Frivate Orchrs.

Common in swampy ground. A small-flowered form of this species has sometimes been taken for $I$. psycodes, a species not as yet discovered on the Island.

Fomna albiflora.

Flowers pure white. Hadlock Upper Pond (B. E. J. Gresham); - Long Pond meadows (Redfield).

CYPRIPEDIUM, L. 'LADY'S SLIPPER.

\section{C. spectabile, Salisb. Showr Lady's Surpper.}

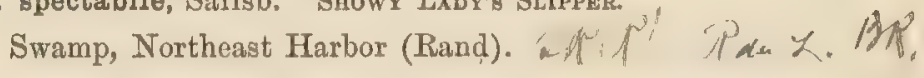

* Not of Hooker. See A. Gray, Ann. Lyc. N. Y., iii. 231.

$\dagger$ H. Hookeriana, Torr, is the more correct name. 
C. acaule, Ait. Strmuess Lady's Stipprr. Common in woods.

Forma albiflorum.

Flowers pure white, or white with indistinct pink -veins. Near Breakneck Ponds (Rand); - Beech Mt. Notch; woods, Hadlock Lower Pond (R. \& R.).

\section{IRIDACE $A$. Iris FamiLx.}

IRIS, L. Blue Flag.

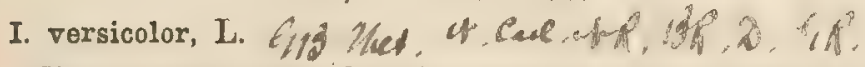

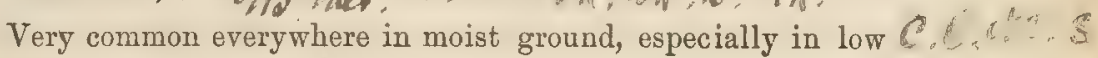
grounds near the coast.

\section{SISYRINCHIUM, L. BLUt-EYed Grass.}

\section{S. angustifolium, Mill. S. Bermudiana, L.*}

Common in grassy places. Whatever may be the fact in re- yhef Peci.D. gard to the specific rank of $S$. anceps and $S$. mucronatum, all the Island forms must be referred to $S$. angustifolium. A specimen with a single spathe, collected by F. M. Day, in 1882, probably in the vicinity of Bar Harbor, has been somewhat doubtfully marked $S$. anceps in the herbarium. A recent and more careful examination, however, seems to show that it is nothing more than $S$. angustifolium with smaller, probably immature seeds. It is worthy of remark that not a specimen of the true $S$. anceps form has yet been found on the Island, although $S$. angustifolium is so very abundant. This fact is evidence that these forms of the plant merit at least varietal distinction.

\section{LILIACEAE. LILY FAMILY.}

\section{HEMEROCALLIS, L。 DAY LILY.}

H. FULVA, I.

Often by roadsides near dwellings; escaped from cultivation. $C A C A$ Town Hill (R. \& R.); - Somesville; Emery District; South
west Harbor (Rand). Adventive from Europe.

* See Morong, Bull. Torr. Bot. Club, xx. 467 . 


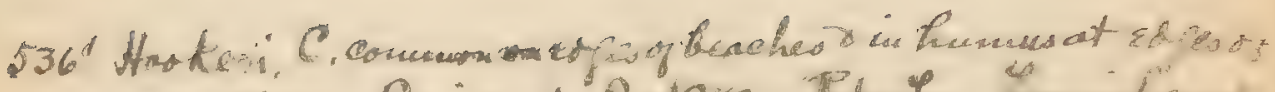

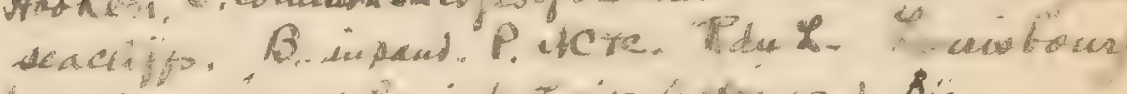

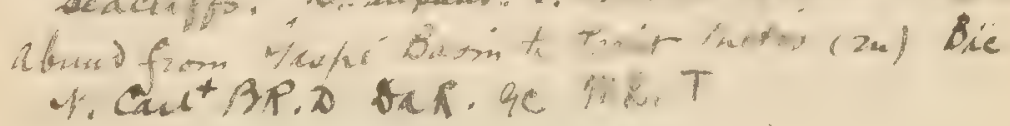

D. Sinontanum $E \mathbb{R}^{+} \mathrm{KC}^{+} \mathrm{AP} \mathrm{P}^{+}$ 

Polygonatum, Adang. Solomon's Seat.

P. biflorum (Walt.), Ell. Smaluer Solomon's Seax.

Infrequent. Head of Little Harbor Brook Notch; woods, Deer Brook; Canada Valley; copses, Long Pond meadows C (Rand); - Hadlock Valley (Redfield); - Rum Key, Porcupine Islands (F. M. Day).

\section{ASPARAGUS, L.}

A. officinalis, L. Gardem asparagus.

Escaped from cultivation; in uncultivated field, Southwest Harbor (Rand). Adventive from Europe.

\section{SMilacina, Desf. False Solomon's Seat.}

S. racemosa ( $\mathrm{L}_{c}$ ), Desf. Falsi Spikmand.

Infrequent. Head of Northeast Harbor; woods, Deer Brook; Northwest Arm woods; Southwest Valley road (Rand); - Hadlock Valley; Browns Mt. Notch (Redfield); - Rum Key, Porcupine Islands (F. M. Day). Tuee $7 x+(1800)$

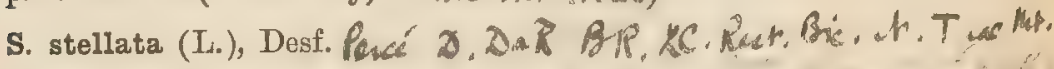
Very rare. Porcupine Islands (F. M. Day) $e^{+} T$

S. trifolia (L.), Desf.

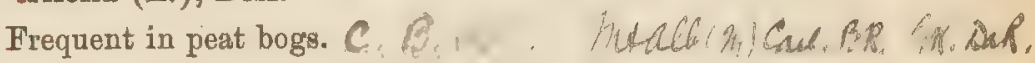

\section{MAIANTHEMUM, Wiggers.}

M. Canadense, Desf. Smitacina bifolia, Ker., var. Canadensis, DaR. H. Gray. Dwarf Solomon's Seat. te

Common everywhere in woods, copses, and clearings.

$$
\text { Cimm }(m)
$$

Streptopus, Mx. Twisted Stali.

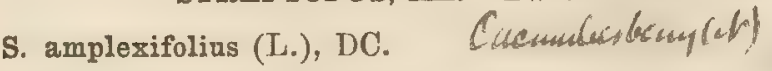
Frequent in deep woods, especially along mountain brooks.

S. roseus, $\mathrm{Mx}$.

Frequent in deep woods. Perhaps more common than the last.

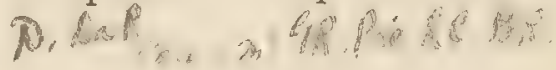




\section{Oytorace (GB)}

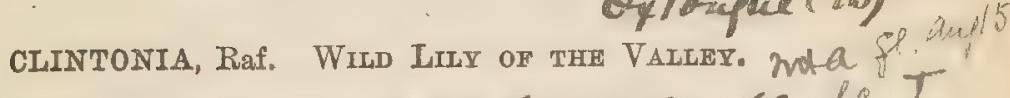

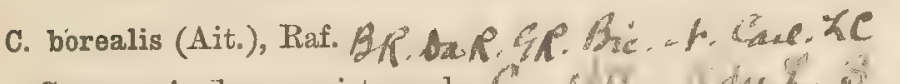
Common in deep, moist woods. $C$. 6 . Wes $A_{n}$. Con

OAKESIA, S. Wats.

0. sessilifolia (L.), S. Wats. Uvularia sessilifolia, L. Common BELLWORT.

Common in deciduous woods and in copses.

ERYTHRONIUM, L. DOG-TOOTH VIOLET.

E. Americanum, Ker.

Rare. Low ground, Clark Point, Southwest Harbor; Fernald Point (Anna H. Bee).

LILIUM, L. LiLY.

L. Philadelphicum, L. Blackberry Lily. Wild Red Lily.

Local, and not widely distributed. Schooner Head (Rand) ; Sargent Mt.; Somesville (R. \& R.); - Norway Drive, and. country about Salisbury Cove (F. M. Day, Clara L. Walley, Mary Minot, Margaret A. Rand).

I. Canadense; I. WILd Yellow Litt. Cakada LiLY.

Rare. Salisbury Cove (Mary Minot). Very common, how- Xetapl ( ever, on the mainland. Ret,

Medeola, L. Indian Cucumber Root.

$\int J 2$ M. Virginiana, L.

Frequent in rich woods.

TRILLIUM, L. WAKE ROBIN。

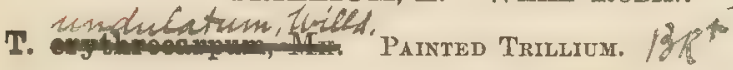

Frequent in damp woods. Sreet $10 \mathrm{~min}(\mathrm{~m})$. ic 
Comalaisa majalis 9 As.

Lilium Bic.

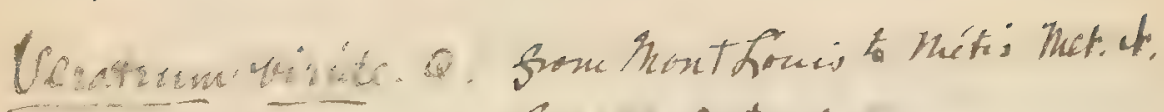

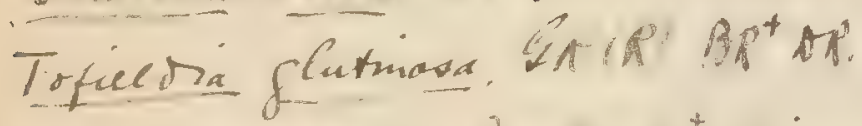

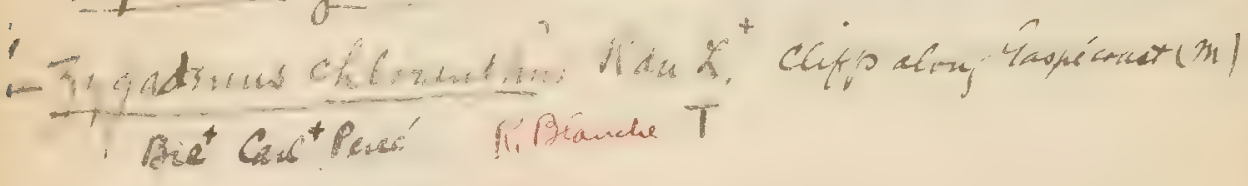

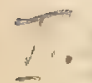

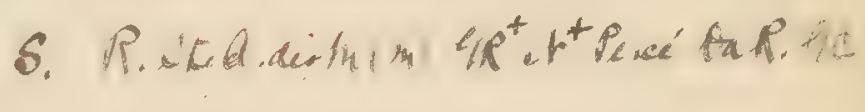
$R \leq a$

- Tercetum.t. t $^{+}$ 


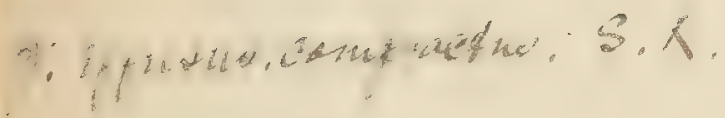

7. tinfisus $7 x+a c b(m)$

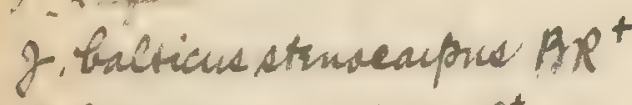

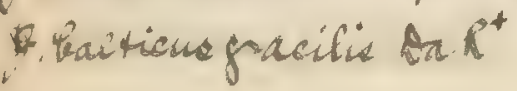

4. 
PONTEDeriace A. Pickered-weed Familt.

PONTEderia, L. Pickerel-Weed.

P. cordata, L.

Streams and muddy pond shores; common. Forms are sometimes found corresponding to var. angustifolia (Pursh), Torr.

\section{XYRIDACE $\mathbb{E}$. Yhliow-eyed Grass Family.}

XYRIS, L. YELLOW-EYED GRASS.

X. flexuosa, Muhl., var. pusilla, Gray.

Peat bogs and sandy shores; rare and local. Hadlock Ponds (Wm. H. Dunbar, Rand);-Breakneck Ponds (A. H. Smith, R. H. Day); - Jordan Pond (T. G. White, Redfield, Annie M. Rand). It is possible that this may be distinct from $X$. flexuosa, and entitled to specific rank. See Bull. Torr. Bot. Club, xix. 38, where this plant is described as Xypia montana, Ries, $-X y r i a$ being an evident typographical error for Xyris. The specific name montan $\alpha$, there chosen, is most unfortunately inappropriate.

\section{JUNCACE E. RUSH FAMILT.}

JUNCUS, L. RUSH.

\section{J. effusus, L. Common Rush.}

Very common in wet ground. $6, \mathbb{S} . \ldots$

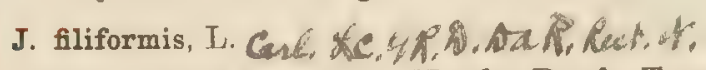

Rare. Edge of pool on Heaths Brook, Tremont, near head $C \in \sqrt{ } C_{W}$ of the Marsh (M. L. Fernald).

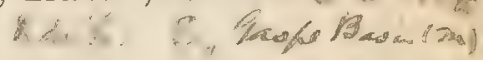

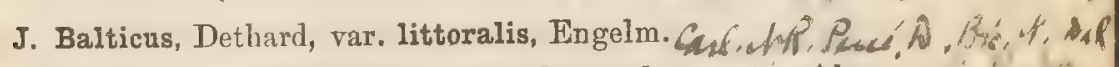

Common in wet brackish ground on the coast. Also on

White Beach, southern end of Great Pond (Rand). $\quad 5 B R$.

J. Greenei, Oakes \& Tuck.

Dry ground; rare. Sargent Mt. (Rand); - Southwest Har. C bor (M. L. Fernald). 
J. tenuis, Willd.

Fields and roadsides; very common.

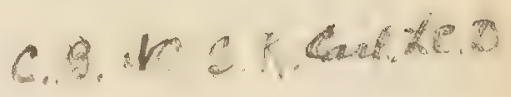

for. secundus (Beauv.), Engelm.

Rare. Dog Mt. (Rand).

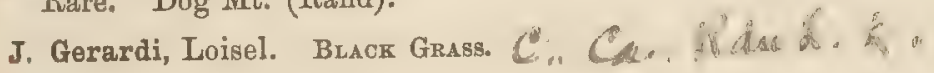

Salt marshes; common.

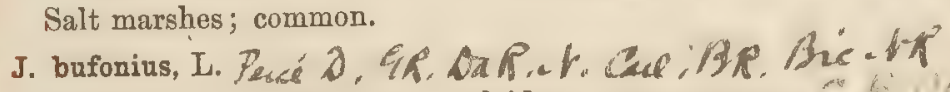

Low ground, especially by roadsides; very common.

J. pelocarpus, E. Meyer.

Wet meadows and pond shores; common. A proliferous $S$, form, Denning Pond (Rand).

J. articulatus, L.

Wet grounds and roadsides; frequent. Northeast Harbor (Greenleaf, Rand); - Somesville (Redfield) ; - Southwest Harbor; High Head (Rand). Foy $\mathrm{h}(\mathrm{m})$ dret a Genfils

$\therefore \ldots$.

J. militaris, Bigel.

Common in streams and ponds.

J. acuminatus, $\mathrm{Mx}$.

Mt. Kebo (Greenleaf); - near Long Pond (Redfield). Probably not uncommon, but as yet seldom reported.

J. Canadensis, J. Gay, Joncicawdatrongetn.

Frequent. Great Head (Redfield); - Southwest Harbor; $气$, Hadlock Upper Pond; Denning Pond (Rand); - Cranberry Islegs Somesville (R. \& R.). L $A$

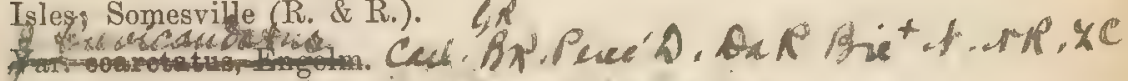
$\because$ Very common. BR

LUZULA, DO. WOOD RUsH.

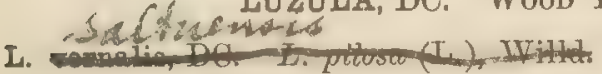

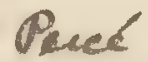

Infrequent. Clearing north of Porid Heath; meadow between Somesville and Town Hill (Rand); - clearings on Indian Point road, Somesville (R. \& R.).

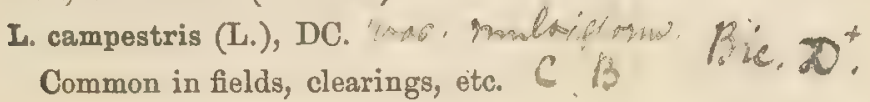




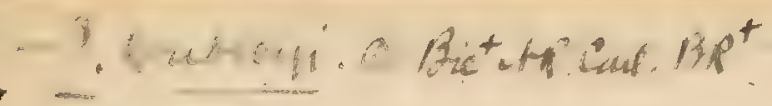

Pi. Bhande

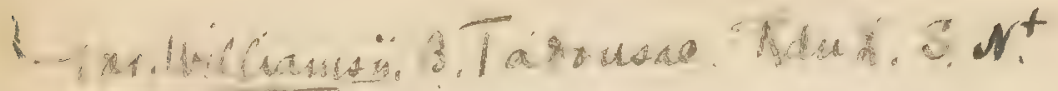

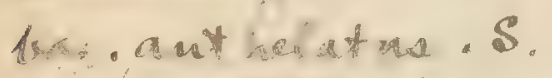

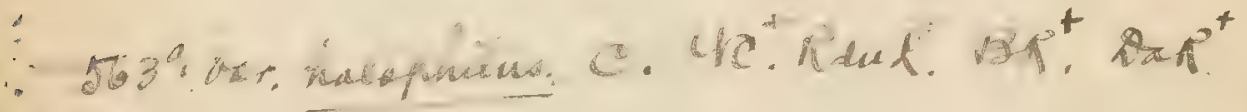
mataue

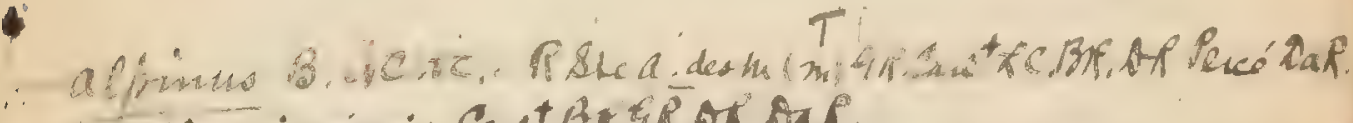

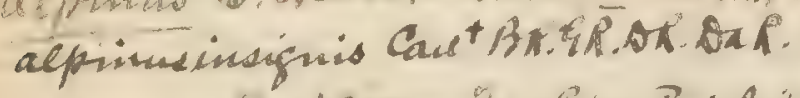

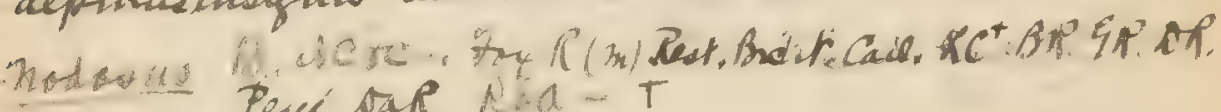
Pence PaR. Nia-T norousen acpinus $O R^{+} A R^{+}$

$\therefore$ Genalugeglianor 5 .

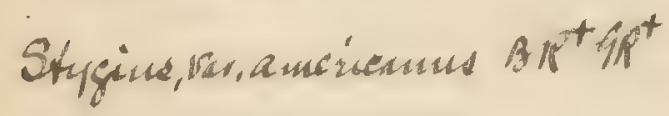

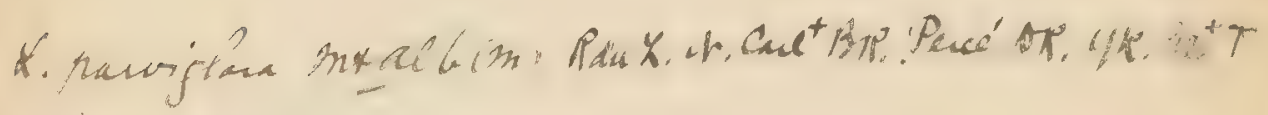

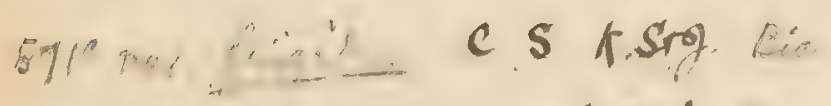

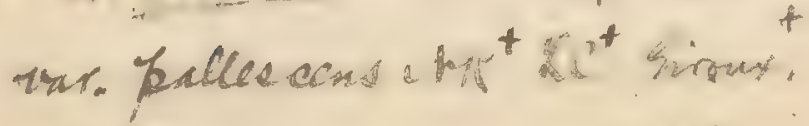

L. opreata, uar. 


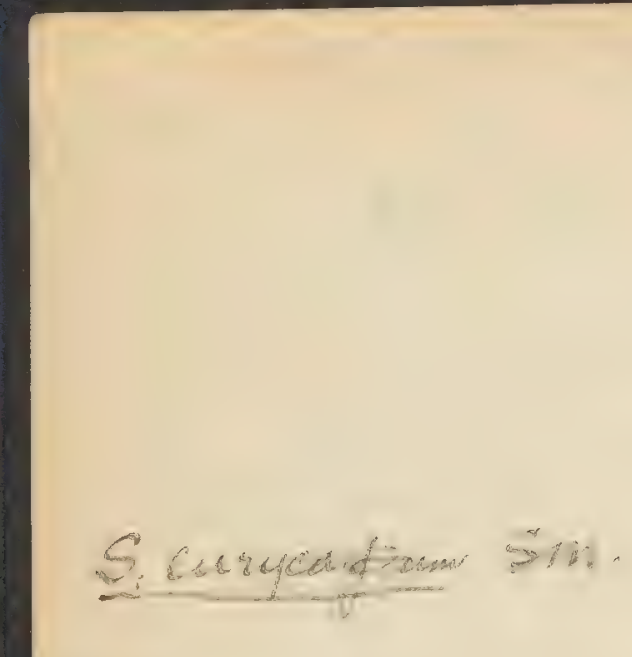

is $\quad 3$ 


\section{TYPHACE $\nexists$. Cat-Tail Family.}

TYPHa, L. Cat-tail Flag.

\section{T. latifolia, L.}

Bogs and marshes; frequent. C. 6 tc $S$ t.

\section{SPARGANIUM, L. BUR-REED.}

\section{S. simplex, Huds. Ax R. $\mathrm{KC}^{+} \mathrm{D}^{+}$}

Common in shallow water, in brooks and ditches. Very variable.

\section{S. androcladum, "zrngerm.}

Common. From a study of Mt. Desert specimens it appears better not to recognize this variety as entitled to specific rank. There is nowell defined dividing line between it and $S$. simplex, and gegasionally the two forms appear on the same plant.

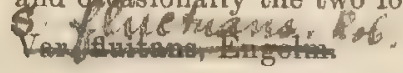

-Floating in water of moderate depth. Somes Pond; Southeast Creek, Bass Harbor (Rand); - Witch Hole (Redfield).

Var. angustifolium (Mx.), Engelm.

Rare. Seal Cove Pond (R. \& R.); - pool, Hunters Brook (Rand). Apparently also in Hadlock Upper Pond (Rand).

S. minimum, Fries.

Ponds and streams; frequent. The Barcelona; Hadlock Upper Pond, etc. (Rand); - Jordan Stream (R.\& R.); - B f f $^{+}$ Northeast Creek (M. L. Fernald).

\section{ARACE E. ARUm Family.}

ARIS.EMA, Mart. INDIAN TURNIP.

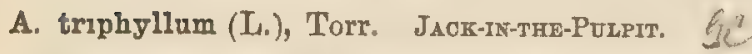

Rich woods, boggy places, and meadows; infrequent. Jordan Pond trail from Northeast Harbor; The Barcelona meadow; Northeast Meadow (Rand); - roadside, north of Doctors Creek (R. \& R.). 
CALLA, L. WATER ARUM.

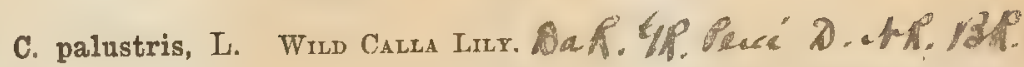
Rare. Near Northeast Harbor (F. I. Temple); - The Heath, Great Cranberry Isle (Arnold Greene, Redfield).

\section{STMPLOCARPUS, Salisb. Skunk Cabbage.}

\section{S. fœtidus (L.), NuTr.}

Swamps; rare. Cranberry Isles (H. C. Jones, Rand, Redfield); Sea Wall and vicinity (Rand). So far as at present $f$ known the distribution of this species is very peculiar. There seems to be no good reason why it should be confined to such a limited area. It may be noted that a slight.elevation of the coast line would connect the Cranberry Isles, where it. occurs most frequently, with Mt. Desert Island at the Sea Wall and vicinity, where it also occurs.

\section{ACORUS, L. Sweet Flag.}

\section{A. Calamus, I.}

Low grounds; rare. Near head of Doctors Creek; near Otter Creek (Redfield); on the "Overflow Brook," Somesville. (R. \& R.).

\section{ALISMaCe E. Water-Plantain Familt.}

SAGITTARIA, L. Arrowhead.

\section{S. variabilis, Engelm.}

Common in shallow water or wet places. Very variable; the various so-called varieties or forms apparently passing into one another by intermediate forms.

Forma hastata.

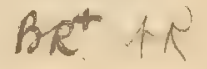

\section{Common.}

Farma obtusa.

Somes Stream (W. H. Dunbar, R. \&.R.). 
finun ruviex s\%

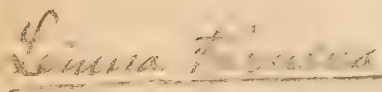

Haber ${ }^{2}-1=5$.

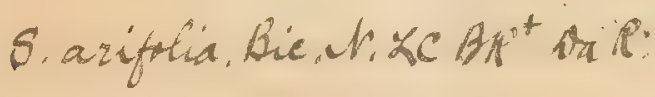


$587 . \mathrm{C}$

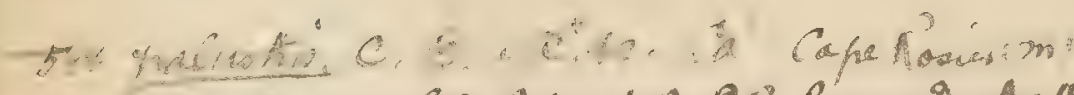

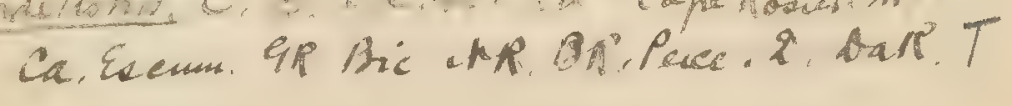

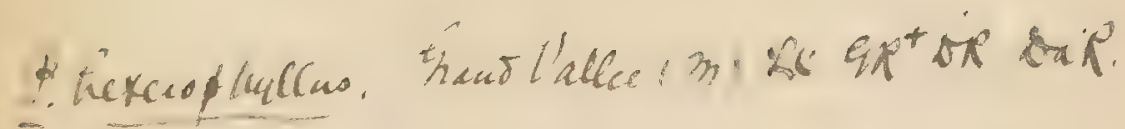


TS Forma angustifolia. Near Bar Harbor (F. M. Day); - Seal Cove Pond; Ripples ‘ N Je
Pond (Rand); - Somesville (R. \& R.).

S. graminea, Mx.

Infrequent. Somes Stream (W. H. Dunbar, R. \& R., Arnold Greene); - Great Pond; Seal Cove Pond (Rand).

NAIADACE AE. Pondweed Family.

TRIGLOCHIN, L. ARrow Grass.

T. maritima, L.T AaR. A. D. AR. GRt Bic. Csemu. UR Common in salt marshes, and on muddy coast shores.

S. palustris, L.

\section{SCHEUCHZERIA, L.}

Rare. Sunken Heath (Faxon, Rand); - The Heath, Great $q p^{+}+1$ Cranberry Isle (R. \& R.).

\section{POTAMOGETON, I. PONDWEED.}

\section{P. natans, $L$.}

Ponds and deep streams; frequent. Seal Cove Pond; Great Pond (Rand); - Somes Pond (R. \& R.); - Northeast Creek; Witch Hole (Redfield). Fuefe Conct (m)

P. Oakesianus, Robbins.

Rare. Northeast Creek; Bubble Pond (R. \& R.).

P. Pennsylvanicus, Cham. P. Claytonii, Tuck.

Common in ponds, streams, and ditches. A very slender deep-water form, Somes Pond (Rand).

P. hybridus, Mx. P. diversifolius, Raf.

Rare. Ripples Pond (Fernald, Rarid). With and without floating leaves.

P. perfoliatus, L.

Infrequent. Long Pond; Jordan Stream (Redfield); - Northeast Creek (E. Faxon, Rand, M. L. Fernald).

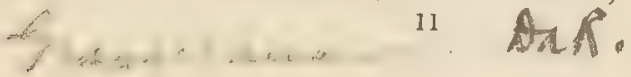


Var. lanceolatus, Robbins. Var, Richardsonii, Bennett.

Rare. Northwest Arm, Great Pond (M. L. Fernald).

P. pusillus, L. AaR.

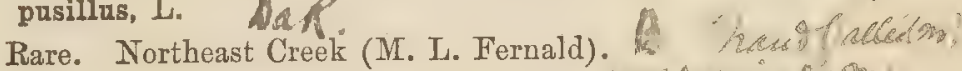

\section{RUPPIA, L. Ditch Grass.}

R. maritima, $\mathrm{L}$.

Ponds and streams of brackish water along coast; common. Plo" RT zOSTERA, L. Eet Grass.

Z. marina, I.

Very common in shoal water along the coast. $/ 4$.

Axil Pie. Carl.

NAIAS, L. Natad.

N. flexilis (Willd.), Rostk. \& Schmidt.

Rare. Ripples Pond (Rand).

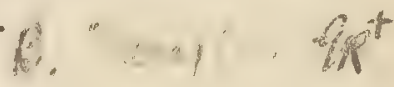

ERiocaule á. Pipewort Family.

ERIOCAULON, L. PipeWORT.

E. septangulare, With.

Common in ponds and along pond borders. Sometimes in deep water, sending to the surface scapes six to ten feet in length, as in Jordan Pond (R. \& R.).

CYPERACE $Z$ E. Sedge Famitr.

DULICHIOM, Pers.

D. spathaceum (L.), Pers.

Frequent on borders of ponds and streams.

Gotherecharis, R. Br. SPIKe Rush.

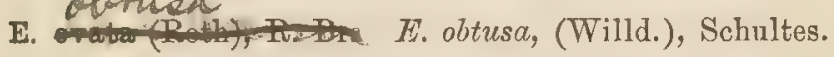

Uncommon. Muddy margins of mill-pond and of Somes $S K$.

Stream, Somesville; bog-hole, High Head; stream near head of

Southwest Harbor (Rand). 


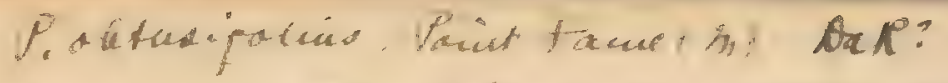

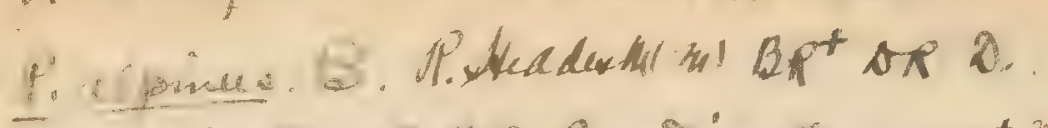

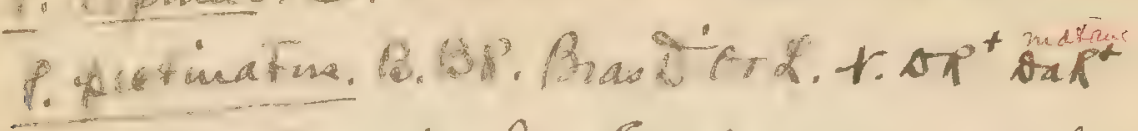

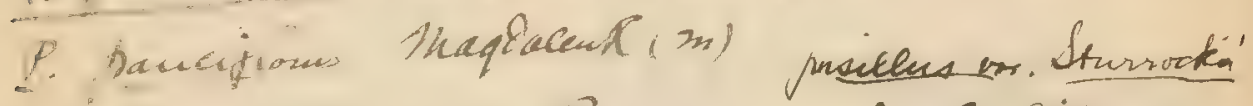

P. marinus Dradsceincle, m!

Dawh. Eivacléz

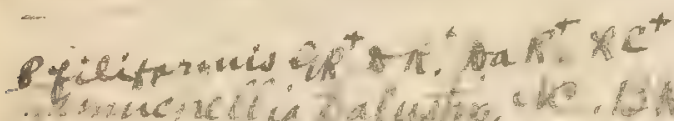

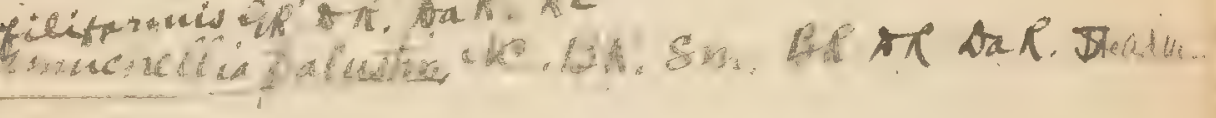

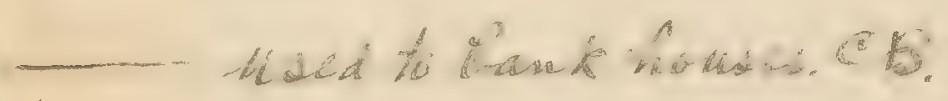


E. intzincia $\operatorname{Sec}^{+} 18 \mathrm{R}^{+} 8 \times 1^{+}$

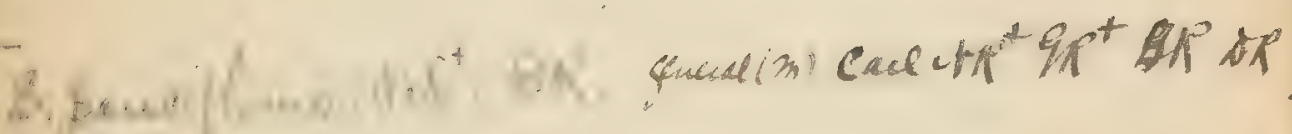
Nar?.

S. vccidentatic Bret 
602 E. olivacea, Torr.

Rare. Sandy margin of Somes Stream (M. L. Fernald).

603

E. palustris (L.), R. Br.

Swamps; infrequent.

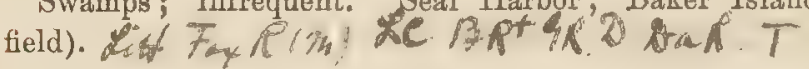

Var. glaucescens (Willd.), Gray.

Very common in marshy ground along the coast. Southwest Harbor; Sea Wall; Somesville; Great Cranberry Isle, etc.

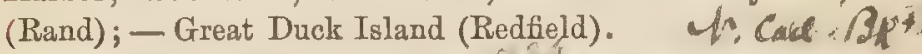
E. tenuis (Willd.), Schultes that Exupl ing if of

Wet ground and pond shores; very common. Variable. $C: Q R$ E. acicularis (L.), R. Br. It A, herthel Cue.

Muddy places; infrequent. Hadlock Lower Pond (Green. $C$. leaf); - Somesville (R.\& R.). Axt.

E. pygmæa, Torr.

Rare. Brackish marsh, Little Cranberry Isle (Redfield). $6 \mathbb{P}^{+} \mathrm{d} A$

\section{SCIRPUS, L. Club Rush.}

\section{$6 \circ 8$ S. cæspitosus, $\mathrm{L}$.}

Local and infrequent. Boggy depressions, Sargent Mt. (R. \& R.); - The Heath, Great Cranberry Isle, - in great abun-

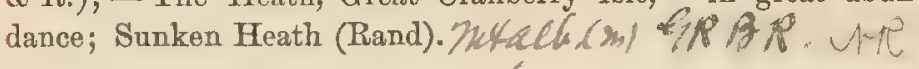

S. subterminalis, Torr.

Ponds and streams : infrequent. Bar Harbor (W. Boott); Seal Cove Pond and Brook; Somes Pond (R. \& R.);-Denning Pond and Brook; meadow at outlet of Great Pond (Rand).

\section{s. punciven, Yaht.}

Rare. Bog on shore south of Sea Wall (Rand). ricisif S. validus, Vahl.

Ponds and marshes; common. In brackish water, Sea Wall the th. (Rand); - Great Cranberry Isle (R. \& R.); - Little Cranberry

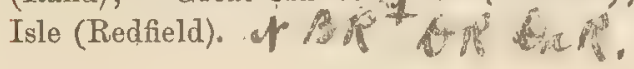


are $N$. ris $x$.

Salt marshes and muddy beaches along the coast; very common.

S. golvaticus, to, - var. dignnus, Boecl. S. microcarpus, Prest.

Wet ground; common. Blooping earlier than the mext. A p. S.A.

S. atrovirens, Muhl.

Wet ground; frequent. Northeast Harbor; High Head; $C_{\text {. A . i }}$ \&, Prettymarsh; Southwest Harbor; Seal Cove, etc. (Rand); Seal Harbor (Redfield). of $\mathrm{R}$.

\section{ERIOPHORUM, L. COTTON GRASS.}

E. cyperinum, I. Scirpus Eriophorum, Mx.

Wet ground; commion and variable.

616 Var. laxum (Gray), Wats. \& Coult.

Frequent. Seal Harbor; Long Pond meadows; Bubble Pond (Redfield); - Southwest Harbor (Rand).

E. alpinum, $L$. A te, $A R^{t}$

Rare. Bog, Northeast Harbor (R. \& R.); - borders of A. I

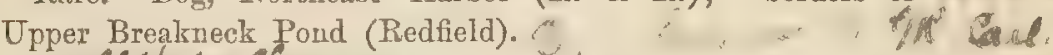
E. Caffish if Cham

Peat bogs and swamps; frequent. Southwest Harbor; Sargent Mt. (Greenleaf, Lane \& Rand); - Little Cranberry Isle;

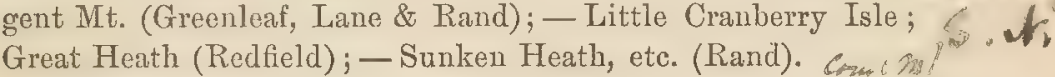

\section{E. Virginicum, I.}

Open bogs and swamps; common. A form bearing unequally $C . S \sqrt{ }$. peduncled spikelets, Great Cranberry Isle (Rand).

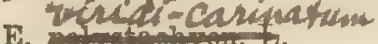

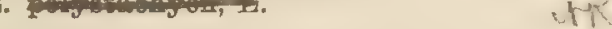

Frequent, Meadow on Sunken Heath Brook (Redfield); Sea Wall Swamp (Rand); - Prettymarsh (Greenleaf, Lane \& Rand).

Var. latifolum (Hoppe), Gray.

More commol than the fpe, - if be varietal listinction is valid. Sargent M. (GreenleakLane \& Rand); - twalls Cove; 


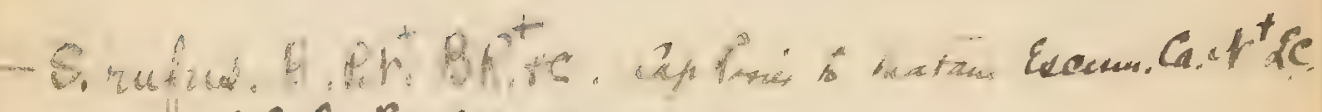
AP. Oal shadu

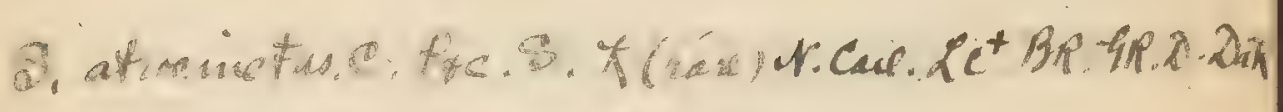

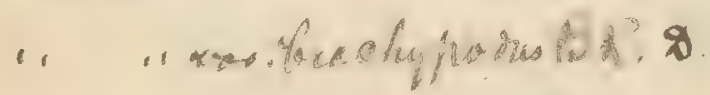

Prereclatio X. Re

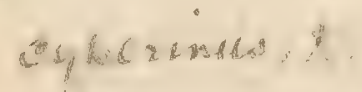

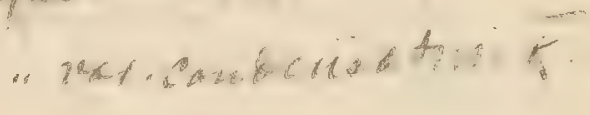

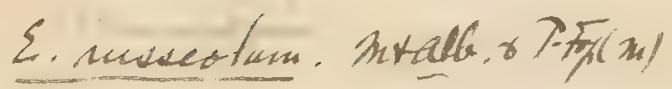

8. Fiolyetachion Ca, Emair Pabo mat. 
6 tenclimm.

$\therefore 5 A^{+} 9 R$.

R.capillaceabst ${ }^{+}$ 
Somesville, etc. (Rand); - Seal Harbor, etc.; Cranberry Isies (Redfield).

622 E. gracile, Koch.

Frequent. Seal Harbor; Little Cranberry Isle (Redfeld); Southwest Harbor; High Head; Great Cranberry Isle (Rand); — "Mt. Desert" (F. M. Day).

RHYNCHOSPORA, Vahl, BeAK Rush.

623 R. fusca (L.), Roem. \& Schultes.

Frequent. Long Pond meadows; Sea Wall (Redfield); ¿K. .... Somes Pond Swamp (R. \& R., Fernald); - Denning Brook; at outlet of Great Pond; Ripples Pond, etc. (Rand).

$62 \%$ R. alba (L.), Vahl.

Wet ground; common.

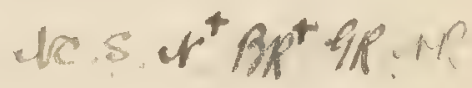

CLAdIUM, P. Br. Twig Rush.

625 C. mariscoides (Muhl.), Torr.

Infrequent. Outlet of Hadlock Upper Pond (R. \& R.); "Mt. Desert" (F. M. Day); - Somesville (M. L. Feruald);swamp above Iong Pond (Redfield).

\section{CAREX, L. SEDGE.}

C. pauciflora, Lightf. yasm. Mirim. mila -

Rare. Borders of Sea Wall Swamp (R. \& R.); - Sunken Heath (Rand). Tryt zcicient is of

C. Michauxiana, Boeckl. C. rostrata, Mx. C. abacta, Bailey.

Rare. Bog, Somes Pond (Rand). Canso, maraut of.

C. folliculata, L. Pieton, ilmax,

Common in swamps and damp meadows.

c. intumescens, Rudge. $\mathrm{A} A \mathrm{C}$ Common in swamps and wet ground. $\$$ he of vigly. ir.anor. qC 
C. oligosperma, Mx. TaGceT\%.

Rare. "Meadow at Gorge, near Bar Harbor" (W. Boott, spec. in Gray Herb.); - "Mt. Desert" (Randall Spaulding); marsh, foot of Lower Breakneck Pond (E. Faxon).

C. utriculata, Boott.

Swamps; common. Heath (R. \& R.).

A form with very narrow spikes, Pond $!$.

Leme.

Var, minor, Boott. Tarecton ac mua

Infrequent. Long Pond meadows (Redfield);-Northeast $A, \boldsymbol{B}, \boldsymbol{C}$.

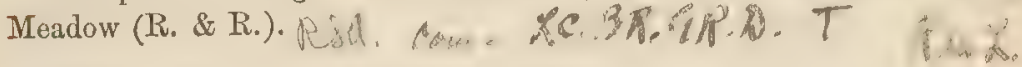

C. Iurida, Wahl. C. tentaculata, Muhl.

Common in swamps and wet ground everywhere. Digley, Hacif, //anu,

C. hystricina, Muhl.

Rare. Meadow on Little Harbor Brook (Redfield). $4 f^{+}, A$, siat 9 .

c. Pseudo-Cyperus, L. Shaur Pú, Pickan, Amuxa

Not uncommon. Seal Harbor (Redfield); - bog south of Sea $12^{4} 5$. Wall; bog at northern foot of Beech Hill (Rand).

C. scabrata, Schw.

Common in wet ground. $Q K, 9 c$

c. Houghtonii, Torr. Xirkifuch

Frequent by roadsides and in clearings. Roadside, Intervale Brook valley; Sea Wall (R. \& R.);-clearings, Youngs District (Faxon \& Rand);-East Peak of Western Mt.; near Oak Hill; road west of Browns Mt. (Rand);-Southwest Harbor (Faxon); - Bubble Pond road (T. G. White).

C. filiformis, $\mathrm{L}$.

Common in bogs and on boggy shores of ponds and streams. An immature form, probably var. latifolia, by roadside south $6 /$. of Salisbury Cove (Greenleaf, Lane \& Rand). com (1/m)

C. fusca, All. C. Buxbaumii, Wahl. $M^{+}$ Rare. Summit of Green Mt. (E. Faxon). 


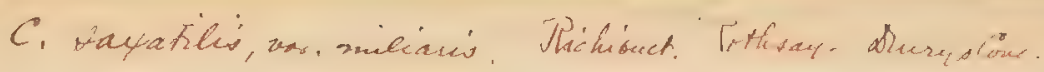

C. Hecicaniar.

$2 x^{+}$

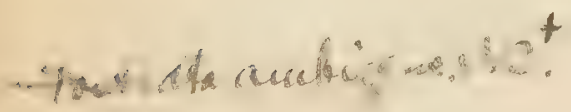

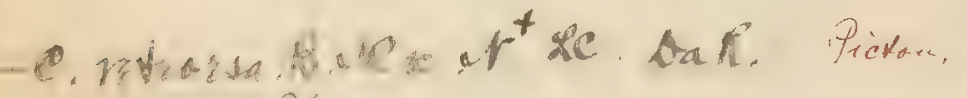

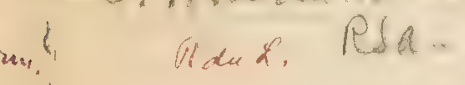

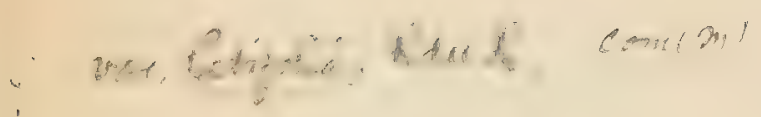

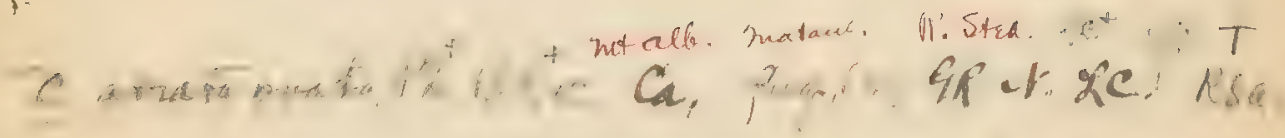

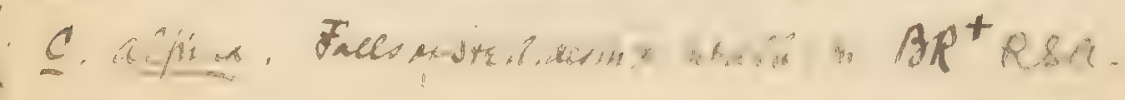


Rigigar thres-

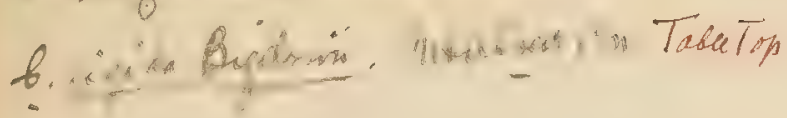

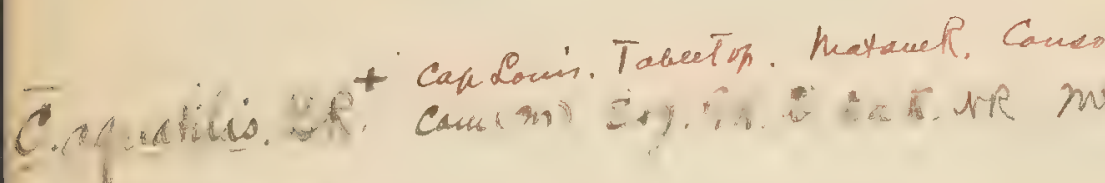
Q nquet. BR.

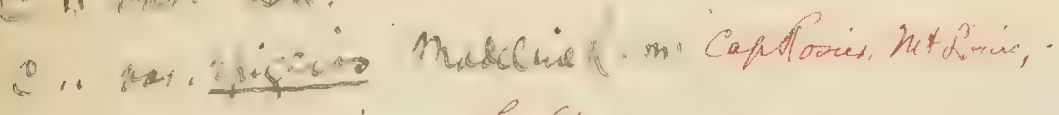
"...r. caspidata, En.Etanf,

b. Glanca. Wiacisor.

G. Torta. Truso \&C.

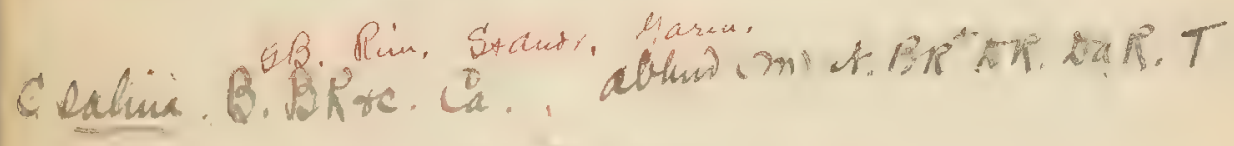

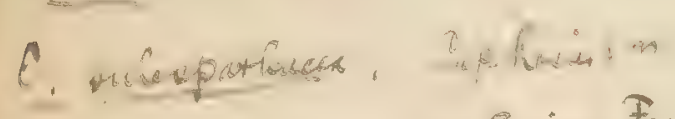

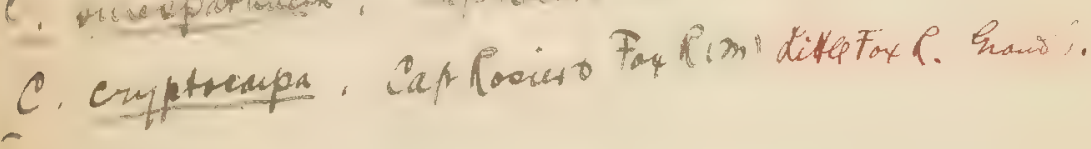

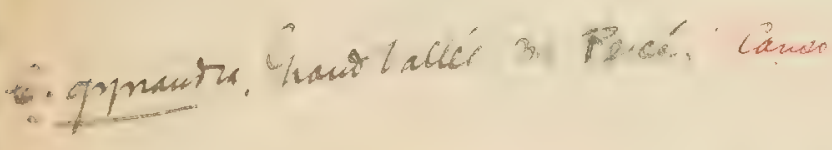

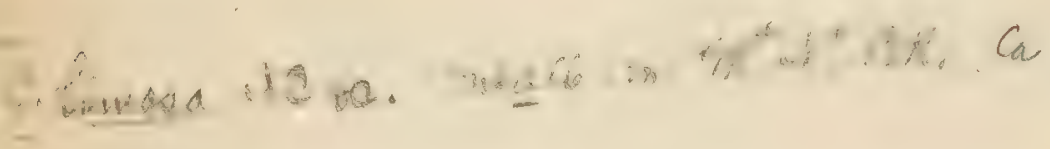

$x=$ maxion mack, TT.

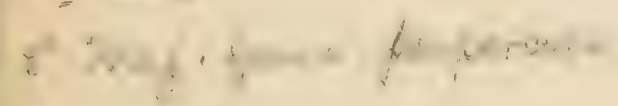




$$
\begin{aligned}
& \text { CATALOGUE OF PLANTS. }
\end{aligned}
$$

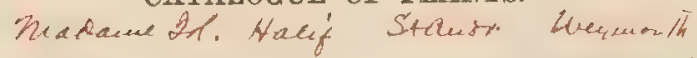

C. vulgaris, Fries. C. rigida, Good, var. Goodenovii (J. Gay), Bailey (Gray, Man., 6th ed., 2d issue, 735 c). Coudo

Common in low grounds; variable. A singular dwarf form, with small very black spikes, Sea Wall (Rand); - Somesville $\mathbf{S}$ (Faxon \& Rand).

intibais that ane

Var. strictiformis, Bailey. C.vigida, Good., var. strictiformis,

Bailey, $l$. $c$.

Frequent. Northeast Harbor (Greenleaf); - Somesville ( $R$. \& R.);-Great Cranberry Isle (Rand); - Northwest Arn, Great Pond; Little Cranberry Isle (Redfeld).

642

c. stricta, Lam. $\gamma_{3}+2, \mathbb{R}$.
Common in wet ground.

11:-int?

Var. angustata (Boott), Bailey.

Infrequent. Intervale Brook valley (Rand); - Somesville and vicinity (Redfield, Faxon, Rand).

Var. decora, Bailey.

Rare. Meadow on Sunken Heath Brook (Rand).

C. lenticularis, $\mathrm{Mx}$. himitinf for.

Gravelly borders of ponds and streams; frequent. Stanley Brook, Seal Harbor (Redfield); - Jordan Pond; Great Pond

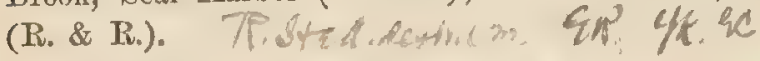

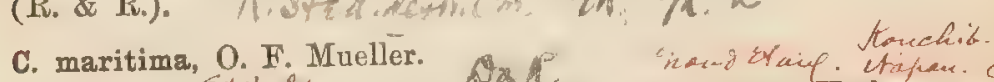

Marshy shores on the coast; common. Southwest Harbor;

Northeast Harbor; Seal Harbor; Otter Creek; Thomas Bay; C Rre. High Head; Seal Cove; Bass Harbor, etc. Fement f.

C. crinita, Lam.

Low ground; common. $C$ chen hovie
Var. minor, Boott.

Rare. On Little Harbor Brook, at crossing of Northeast $C$ Harbor trail to Jordan Pond (Redfield). S. Pich.

c. Magellanica, Lam. C. irrigua, Smith. Camso. Yarue. Sry.

Frequent in cold bogs. Also Green Mt. (Redfield); - Sar-C. B,te. gent Mt. (R. \& R.); - Little Cranberry Isle (Redfield).

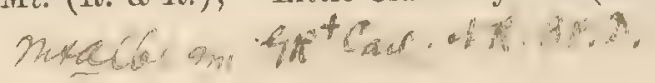




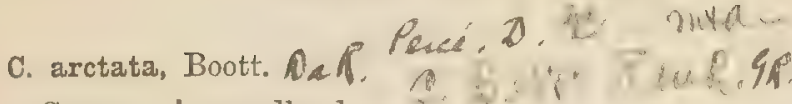
Common in woodlands.

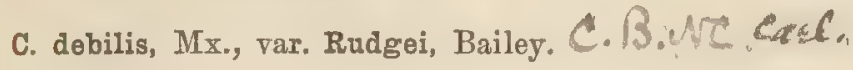
Common. Aifh. Couss, Hal.

C. gracillima, Schw.

Meadows and low ground; frequent. Seal Harbor (Redfield); C. - south of Town Hill; High Head meadow (Rand); - Northeast Meadow (R. \& R.), and elsewhere. Euccum, ev. DxR, ?C

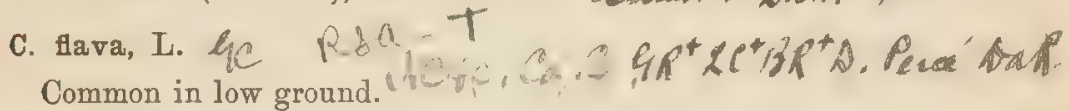
Var. graminis, Bailey.

Uncommon. Shores, Northwest Arm, Great Pond; Ripples _ . $/ / C$ Pond (Rand); - field near Ship Harbor (Redfield \& Faxon); Long Pond meadows (Redfield). Depauperate forms of this variety are found in abundance on shores of Great Pond (Rand). Var. viridula (Mx.), Bailey. C. Ederi, Gray, Man., 5th ed. D , e

Common in both wet and dry ground.

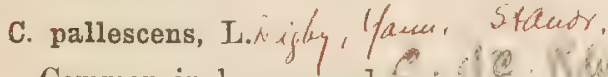

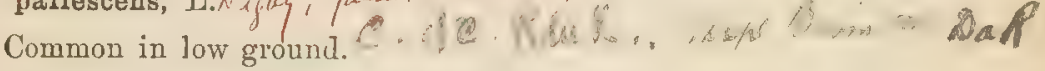

C. conoidea, Schk. Stauor, Camaf.

Low ground about Long Brook, Great Cranberry Isle (Red-C. field, Rand); - Southwest Harbor (M. L. Fernald).

158 c. Iaxiflora, Lam.

Grassy places; common and variable.

Var. varians, Bailey. Qc Mt $A-T$

With the type, and as common.

C. PANICEA, L.

Rare. Low grassy ground at mouth of Long Brook, Great Cranberry Isle (Theodore G. White, Redfield). Appearing indigenous, but probably naturalized here from Europe for many years. 


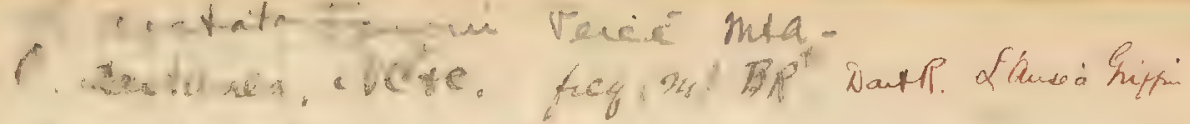

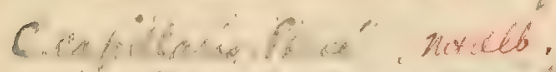

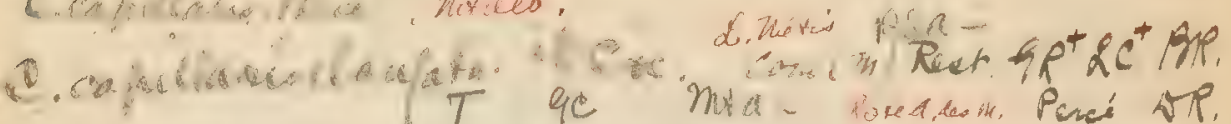
C. Sumedecran.

6. $f$ and

- Coderi itd" apt Pencé e:- is ${ }^{+}+1 \mathrm{t}$

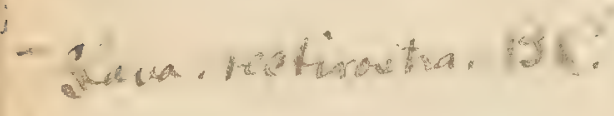

c. Givina $\sin ^{+} \lg ^{+}$

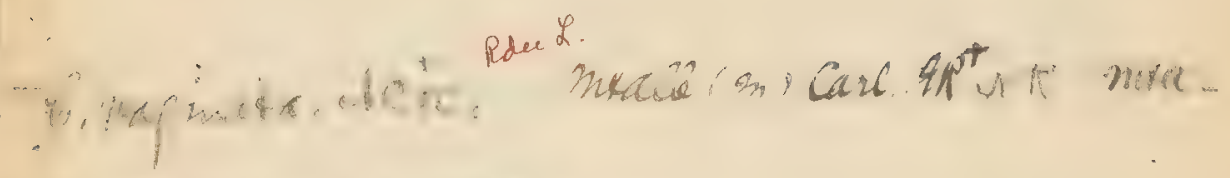
C. Gicolarist 1181

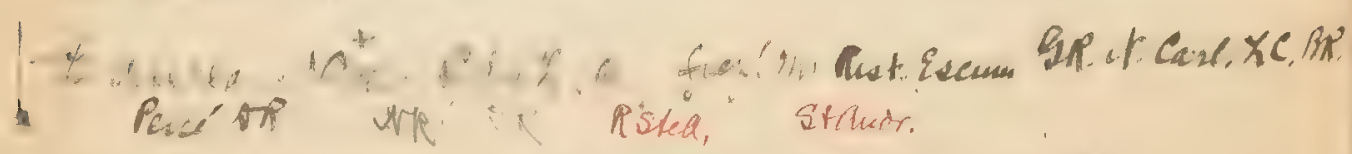

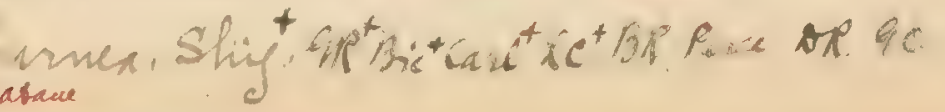




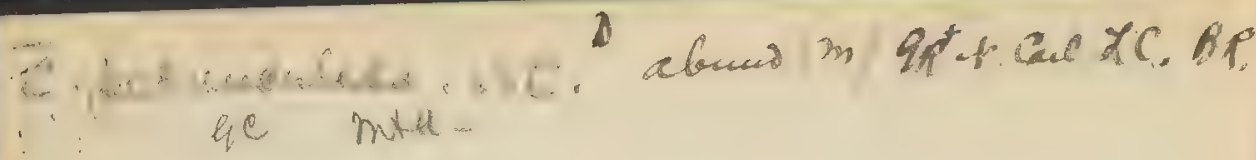

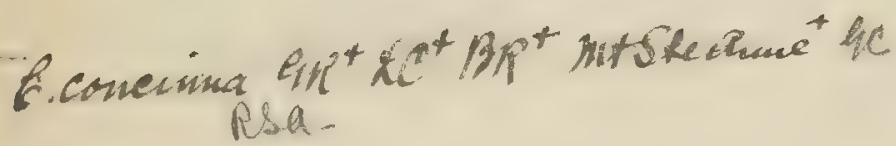

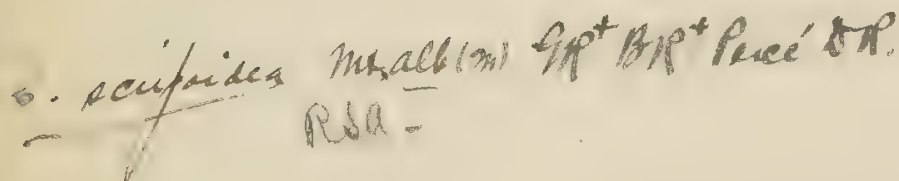

b. allicaur, frea.desm, $1 c^{+}$Resa.

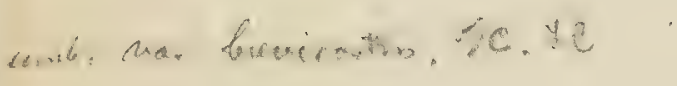


C. deflexa, Hornem. C. Novoe-Anglice, Gray, Man., 5th ed., mostly; non Schweinitz.

Not uncommon on mountains, and in dry ground in woods and clearings. Sargent Mt.; Beech Mt. Notch; Breakneck road; Southwest Valley"road; Sonthwest Harbor; Hadlock Lower Pond; Somesville (Rand). On Town Hill road, Somesville, a form approaching var. Deanei (Rand). mka.

Var, Deanei, Bailey.

Frequent in dry clearings. Somes Pond pastures; Hulls Cove; Beech Hill cross-road (Rand); - Somesville; Seal Harbor (Redfield); - Indian Point road, Somesville (Faxon \& Rand).

C. varia, Muhl, C. Emmonsii, Dewey.

Infrequent. In dry ground, Sutton Island (Redfield); Beech Hill cross-road (Rand); - Norwood Road, Southwest Harbor (Faxon \& Rand).

C. Norm-Anglix, Schw. S, Staut, Stg;

Common, especially in dry clearings. Beech Cliff; Somes $C$ Pond pastures; Interrale Brook valley; Sargent District; Seal Cove; Indian Point road, Somesville; Norwood Road, Southwest Harbor; head of The Barcelona, etc. (Rand); - Breakneck road, etc. (Redfield). Cose. Hy.

\section{Pennsylvanica, Lam.}

Infrequent. Abundant, however, along the Indian Point road between Somesville and Oak Hill (R. \& R. ); - Breakneck road, near Hulls Cove (Redfield).

c. communis, Bailey. C. varia, Gray, Man., 5th ed.

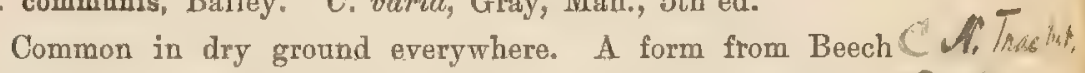
Mt. Notch "approaching var, Wheeleri, Bailey" (Rand). A D. $8 \mathrm{C}$ form from clearing on Meadow Brook, Indian Point road, Somesville, with very soft, short, bright green leaves (Faxon \& Rand). Perigynia of this species much infested by a smut.

C. umbellata, Schk.

"Very low and compact, with the spikes all closely clustered near the surface of the ground." I. H. Bailey, Bull. Torr. 
Bot. Club, xvi. 219. Frequuent. High Head; Browns Mt.; Youngs District, etc. (Rand); -Breakneck road (Redfield).

Var. vicina, Dewrey.

"Looser and taller than the type, with many of the peduncles elongated and becoming true culms." Bailey, l.c. More common than the type, Sargent Mt.; elearings, near Sunken Heath; Intervale Brook valley; Beech Mt. Notch, etc. (Rand); - Somesville; Sargent Mt,; Beech Cliff (R.\& R.). Forms intermediate between this and the type are not in-

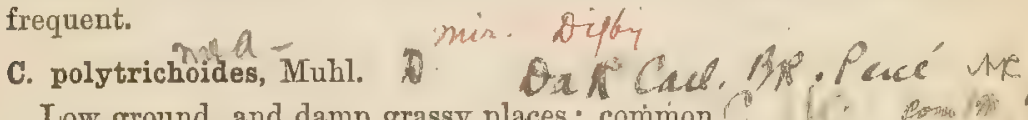
Low ground, and damp grassy places.; common. ( )

\section{c. stipata, Muhl. he RSa gare \\ Very common, and variable, \\ C. tenella, Schk.

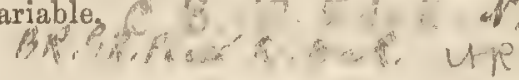

Damp places; infrequent. Sargent Mt; woods on Town $C, 1 \mathrm{C} / \mathrm{h}$

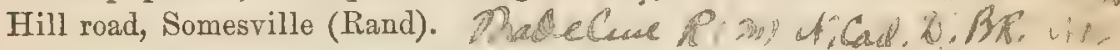

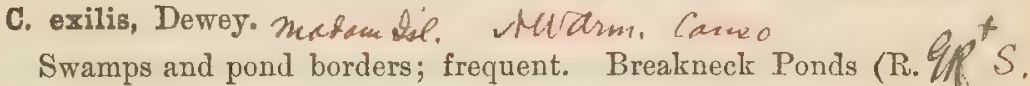
\& R.); - Somes Pond (E. Faxon); - Sunken Heath (Rand). Perigynia much infested by a smut.

c. sterilis, Wille- C. echinata, Murray, var.-micrastachys, Boeckl, Gray, Man., 6th ed., 618.

Short, stiff, and erect (usually not much exceeding $1^{\circ}$ in mothle. beight), the old leaves often persistent; head tawny or greenishyellow, short, composed of from three to five small loosish contiguous spikes, of which the uppermost is usually conspicuously attenuated at the base by the presence of staminate flowers, sometimes the terminal spike, or even the whole head, is entirely staminate; perigynium thin and flat, conspicuously contracted into a slender beak, - which is nearly or quite as long as the body and spreading so as to give the spike an echinate appearance, - sharp-edged and rough on the upper margins, variously nerved and very sharply toothed. I. H. Bailey, Bull. Torr. Bot. Club, xx. 424. Common in bogs and meadows. Head of 


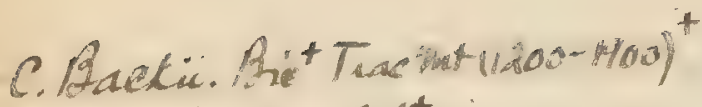

C. rucpionsidea $C_{N^{+}}$

- C.eluoworlaja $\mathrm{er}^{+}$

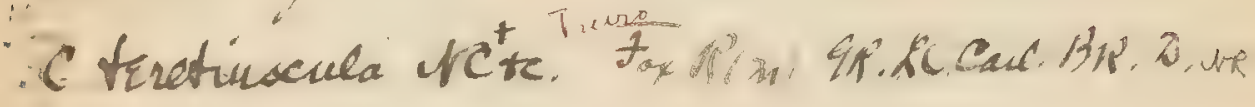
C. furirea Bnit

C. inea, unv nodiata of het

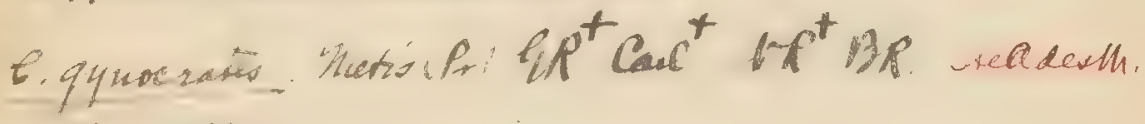
in, Detió 


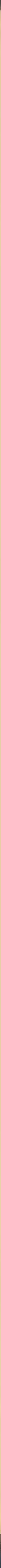


Northeast Creek; Sargent Mt.; High Head, and elsewhere (Rand); - Somesville; Sea Wall (R.\& R.).

Var. excelsior, Bailey: $\$$. Yамu. Caur.

Taller and more slender (often $2^{\circ}$ high), the heads usually $C$ more' scattered and mostly somewhat greener. Bailey, l. c. f
Common in bogs and low grounds throughout the Island.

Var. cephalantha, Bailey. C. echinata, Murray, var. cephalantha, Bailey. Gray, Man., 6th ed., 618. Causo

Rather stiff but slender and tall, or the top of the culm weak $\left(1^{\circ}-2^{\circ}\right.$ high); head mostly continuous or more or less dense and composed of five to eight approximate (rarely seattered), large (15-30-flowered) green or greenish loose spikes, in which the mature narrow long-beaked perigynia usually spread nearly or quite at right angles. 'Bailey, l. c. Frequent. Little Cranberry Isle; Barr Hill; Seal Harbor (Redfield); - meadow, Doctors Brook; High Head meadow (Rand); - Salisbury Cove (R.\& R.).

Var. angustata (Carey), Bailey. C. echinata, Murray, var. T. . angustata, Bailey. Gray, Man., 6th ed., 618.

Very_slender, sometimes almost thread-like, weak, bearing long and narrow divaricate perigynia, which are either in loose small heads or in scattered spikes. Bailey, l. c. Rare. Wet ground at junction of Prettymarsh and Seal Cove roads (Rand).

C. Atlantica, Bailey. C. echinata, Murray, var. conferta,

Bailey. Gray, Man., 6th ed, 618. Mricume'. Caneo

Tall $\left(16^{\prime}-24^{\prime}\right)$ and very stiff and strong, the leaves broad but stiff and usually becoming somewhat involute when dry; spikes contiguous or scattered, spreading, globular or shortcylindrical, densely flowered, green; the terminal one slenderly contracted below or even eutirely staminate; perigynium large and very broad (the body about as broad as long), with a distinct rough, bifid beak, strongly many-nerved, especially upon the back, squarrose or usually retrorse at maturity, shelling off readily when ripe. Bailey, $l . c$. Frequent. Sargent Mt.; Freeman Heath; meadow on Sunken Heath Brook; wood road to Aunt Bettys Pond (Rand). None of the specimens thus far collected are really typical. 
c. canescens, L. Rla -

Wet grounds ; common everywhere. Forms approaching $C, C, C$. var. alpicola, Wahl., occur on Indian Point road, Somesville; Vhe in woods near Spruce Point, Eden; and near northern end of l'i.er Denning Pond (Rand).

Var. vulgaris, Bailey. Muram

Common as the type; in woods and drier places.C. Pene C. Norvegica, Willd. Enanci Etan

Rare. Borders of salt marsi, Little Cranberry Isle (Red- $D$. I

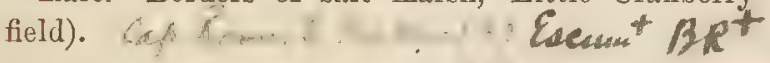

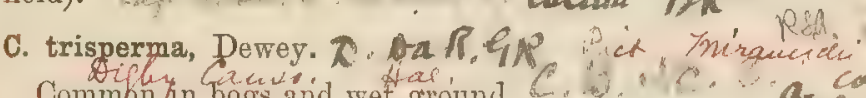

Commpn in bogs and wet ground. $C$. A

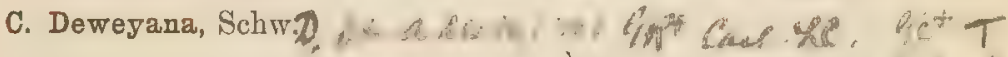

Rare. Southwest Valley road (Greenleaf, Lane \& Rand). i A

C. tribuloides, Wahl. Cantivecefim

Northeast Meadow (R. \& R.); - burnt woods, Youngs District (Rand), Commonly appearing in the next named variety.

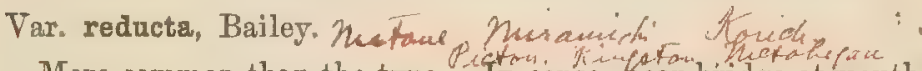

More common than the type. Prin copse, hear bridge at mouth of Northeast Creek; High Head meadow; Oak Hill; bog at 1 dus $\mathrm{S}$,

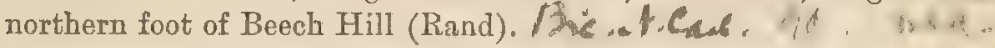

Var, cristata (Schw.), Bailey. C. cristata, Schw.

Rare. Damp roadside at northern foot of Beech Hill (Rand).

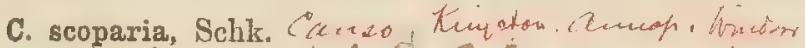

Commoz everywhere. C. S. S. .

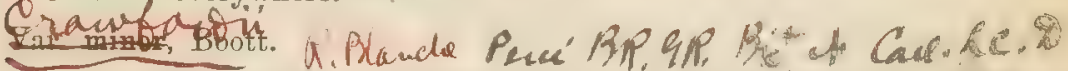

Dry ground; infrequent. Beech Mt. Notch; Youngs District; Somesville; road west of Browns Mt. (Rand). "C.

C. adusta, Boott. C.pinguis, Bailey, not C. adusta of Gray, Man., 5th ed. Sacuont, Keurc.

Local, but not uncommon. Mt. Kebo (Greenleaf); - burnt woods, south of Sunken Heath (Faxon \& Rand); - Sea Wall fe. he, road, Southwest Harbor; Intervale Brook valley; road west of Browns Mt. (Rand). 
- Hheluaca, B.

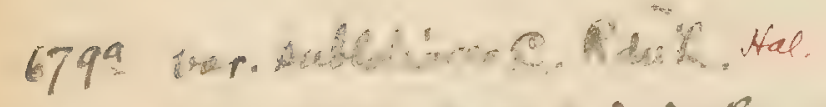

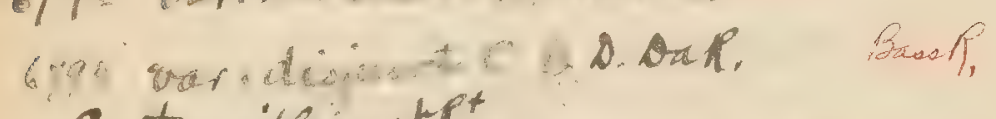

c. teunifiond $t \mathrm{R}^{+}$

c slareosa.

Hic $*$ s

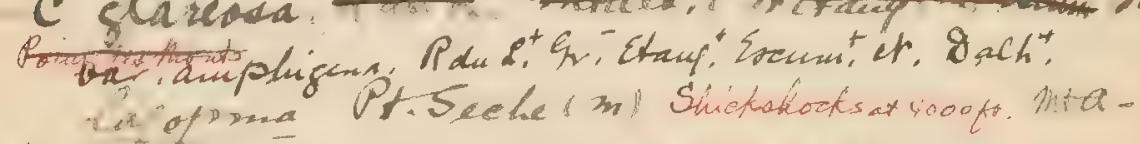

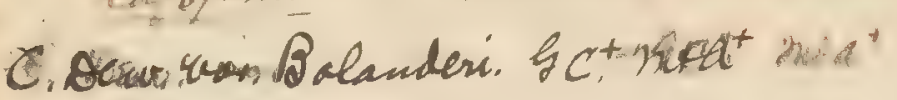

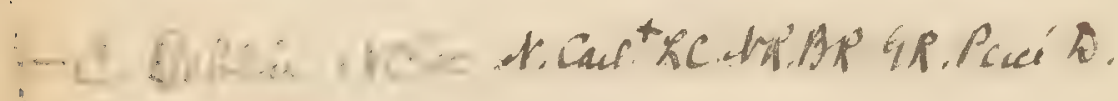

P. Slauche

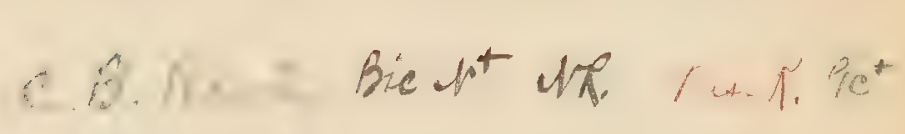
Y6 19. 


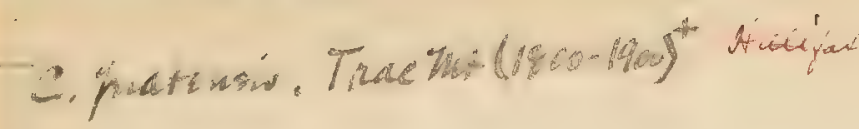

b. íparina. Yasmonth 
689 C. foenea, Willd. C. adusta of Gray, Man., 5th ed. Cace. Dry ground; conmon.

Var. perpleza, Bailey.

Rare. Somesville (Redfield); - Beech Hill (Rand). * to

C. silicea, Olney. Mirmen, Saber 9

Rare. Beach near Thumbcap, Great Cranberry Isle (R. \& R.). 5 †

C. straminea, Willd. C. straminea, var. tenera of Gray, Man., $C$ 5th ed.

Infrequent. Woods, Seal Harbor (Redfield); - meadow on Denning Brool; Somesville (Rand).

693 Var. brevior, Dewey.

Infrequent. Mt. Kebo (Greenleaf); - Seal Cove (Rand); Town Hill (M. L. Fernald). A depauperate form, Newport Mt. (Rand).

Var. aperta, Boott. Bass $\mathbb{R}_{0} \hat{R}$, Muatave

Common in wet ground, especially near the coast.

Var. invisa, W. Boott.

his ti $^{\circ}$

Rare. Schooner Head (W. Boott, spec. in Herb. Gray); bog on shore south of Sea TVall (Rand); - Southwest Harbor (M. L. Fernald), A form nearly approaching this variety, Bass Harbor (Rand). Buidest. Pietau

Var. alata (Torr.), Bailey. .C. ulata, Torr.

Seal Harbor (Redfield). Specimens not entirely characteristic, but apparently this variety.

C. albolutescens, Schw. Cr. straminea, Willd, var. foenea, Torr. Gray, Man., 6th ed., 622.* Mirdme

Infrequent. Road west of Browns Mt. (Rand), and probably elsewhere.

Var. cumulata, Bailey. C. straminea, Willd., var. cumulata, Bailey. Gray, Man., 6th ed., 622.

Dry ground, especially in newly disturbed soil; common.

* See also Bailey in Bull. Torr. Bot. Club, xx. 421, 422. 


\section{GRAMine A. Grass Family.}

SPARTINA, Schreb. MARSI GraSS.

s. cynosuroides (L.), Willd. GaR. T

Frequent on salt marshes. Also Beech Hill (Rand); where seed was doubtless introduced in seaweed used for field dressing.

S. juncea (Mx.), Willdy SALT RUSH Grass.

Salt marshes and sea beaches; common. $S_{4}, C_{a}$

S. Atricta (Ait.), Roth, var. glabra (Muhl.), Gray. SALT Marsi Grass.

Common on muddy shores along the coast, and in salt marshes. Also Cranberry Isles. A form intermediate between this and the next variety, Little Cranberry Isle (Redfield).

Var. alterniflora (Loisel.), Gray.

Rare. Head of Somes Sound (Redfield); - Norwood Cove (M. L. Fernald). Cuect 0 , Ale

PANICUM, L. Panic Grass.

\section{P. capillare, L.}

Uncommon. Shore, Ripples Pond; field on Great Pond road, Southwest Harbor (Rand). Perhaps introduced on the Island.

P. commutatum, Schultes.

Rare. Field, Northwest Cove (Rand). "Although this plant differs much from southern specimens of $P$. commutatum, it is hard to place it elsewhere than in this species." $F$. Lamson-Scribner.

P. nitidum, Lam.

East Peak, Western Mt. ; Southwest Harbor (Rand).

Forma densiflorum.

An unusual form having panicles somewhat densely manyflowered. Shore of Ripples Pond (Rand). 


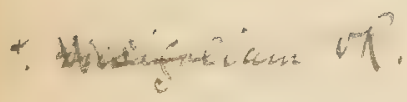

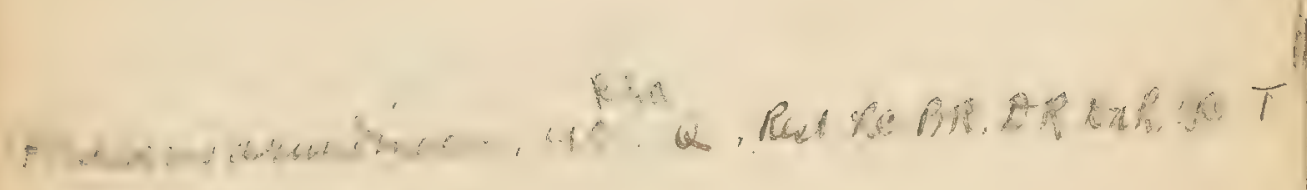


P. depauperatum, Muhl.

Roadsides and clearings. Somesville (R. \& R.); - near Sunken Heath; Sheep Island, Somes Harbor (Rand).

P. dichotomum, $L$.

Common both in dry and damp ground. Exceedingly variable, appearing certainly in forms commune and fasciculatum.

P. 1axiflorum, Lam.

Frequent. High Head; Somesville (R. \& R.); - Beech Hill road, Southwest Harbor (Rand). A smooth form (P. laxiflorum, Chapm. Flora S. States, Suppl. 667), Northeast Meadow (Rand).

P. Crus-galli, L. Barsyard Grass.

Frequent in barnyards and cultivated ground. Appearing in both long-awned and awnless forms. Naturalized from Europe.

Setaria, Beauv. Bristly Foxtail Grass.

S. giaduca (L.), Beauv, Foxtail Grass.

Cultivated ground. Southwest Harbor; High Head; Seal Harbor (Rand). Adventive from Europe.

S. viridis (L.), Beaur. Green Foxtail.

Common in cultivated grounds and waste places. Naturalized from Europe.

S. Italica (L.), Roem. \& Schultes. Mrtlet.

Head of Northeast Harbor, in grass plots (Rand). Adventive from Europe. The form is that known as S. Germanica (L.), Beauv., distinguished from the type by its more dense or compact, and usually erect, panicle or "head."

LeErsia, Swz, Cux Grass.

L. oryzoides (L.), Swz. Rrcm Cut Grass.

Border of Somes Stream (Rand); - Seal Cove Pond meadow (R. \& R.). 
ANTHOXaNTHUM, L. Sweet Vernal Grass.

A. odoratum, L.

Fields and meadows; becoming common. Naturalized from " Europe.

HIEROCHLOE, Gmelin. SwEET Grass.

H. borealis, Roem. \& Schultes. A N R R R . AP. Ric of

Borders of salt or brackish meadows and marshes. Bass Harbor; Southwest Harbor; Little Harbor; Seal Harbor; $C$ Northeast Meadow; Thomas Bay; Cranberry Isles; Duck Islands, and elsewhere. Much used by the Indians for basket work. nkacl son:

STIPA, I. Feather Grass.

S. Macounii, Scribner." S. Richardsonii, Gray, non Link. Gray, Man., 6th ed., 641.

Rare. Burnt woods, Youngs District (Rand, E. Faxon); wood clearings, Somesville (M. L. Fernald).

ORYZOPSIS, Mx. Mountain Rice.

0 . asperifolia, $M x$.

Dry woods and clearings; not uncommon. Theldeic $P(m)$ Pie.

MUHLENBERGIA, Schreb. Drop-seed Grass.

M. glomerata (Willd.), Trin.

Infrequent. Damp field, Northeast Harbor (Rand); - Long $A$. A Pond meadows (Redfield); - Sargent Mt.; Beech Mt. Notch; FP s scé shore, Jordan Pond (Rand). Muedelius $P$, m/ Twe hut (1500-Hool

* M. diffusa, Schreb.

"In abundance," western slope of Green Mt. (Arnold Greene).

BRACHYELYTRUM, Beauv.

B. aristatum (Pers.), Beauv.

Low woods throughout the Island; frequent, but not abundant. Pdu $f$

* Bull. Torr. Bot. Club, xix. 154. 
Pt, al/ama, nuta-

Cipgenatzois. Bic.

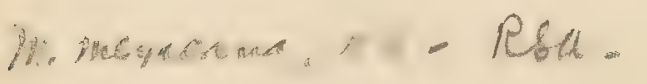




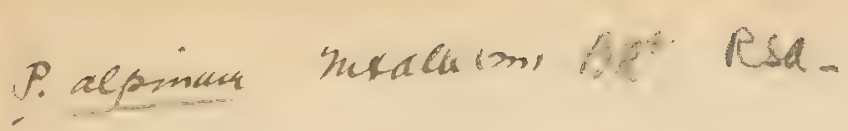

Aepopinumatu. S. Sog

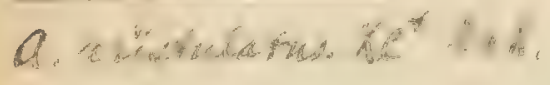


phliedm, L. Cat's-tail Grass.

P. PRATENSE, L. TimothY.

722 Common in fields and meadows. Naturalized from Europe.

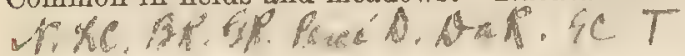

\section{ALOPECURUS, L. Fox-TAIL Grass.}

A. pratensis, L. Mendow Fox-tair.

Fields and by roadsides; infrequent. Seal Harbor (Redfield); $C, S$, - Southwest Harbor ; Bar Harbor ; Hulls Cove; Somesville (Rand). Naturalized from Europe.

- SpORobolus, R. Br. Drop-seed Grass.

S. serotinus (Torr.), Gray. Misr Grass. $C$ ": Meadows and low ground; frequent.

\section{Agrostis, L. Bent Grass.}

A. alba, L. White Bent Grass. Herd's Grass. A $\lambda$, ef Cul Xe, BR, Meadows and fields; common. Naturalized from Europe through cultivation. Tenet, D. T

6 Var. stupatica (L.), Scribner.

A viviparous form of the species. Beech Hill; Somes Stream $;$. (Rand).

727 Var. coarctata (Hoffm.), Scribner.

Panicle dense; branches short, flower-bearing to the base; plant not stoloniferous. In wet ground; frequent. Baker Island (Redfield); - High Head; bog, Kings Point, Southwest Harbor (Rand); - head of Somes Sound (Greenleaf). Doubtless indigenous.

Var. stolonifera (L.), Vasey.

Panicle dense, but the spikelets less crowded than in var. coarctata, narrow, often linear; plant stoloniferous. On beaches by the shore, and elsewhere. Hunters Beach; High Head (Rand) ; - Little Cranberry Isle; Little Harbor (Redfield). Doubtless indigenous. 
Var. vULGARIS (With.), Thurb. ReD-Top.

Meadows and fields; common. Naturalized from Europe $C_{4} / S$ through cultivation, and perhaps also indigenous.

A. perennans (Walt.), Tuck.

Frequent in damp shady places and by brooksides. Beech Mt. Notch; Deer Brook, Jordan Pond; Gilmore Brook; Little Harbor Brook Notch, and elsewhere (Rand);-Southwest Harbor $\partial$ (M. L. Fernald). It seems doubtful whether these northeru plants belong to the true southern $A$. perennans. A form from woods south of Beech Mt. may be $A$. Novo-Anglioe, Tuck.

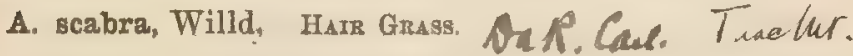

Dry soil; common. A dwarf form growing in tufts in rock $S, A$. hollows and dry places is var. montanum (Torr.). Sargent $\mathscr{D}, \mathscr{A C}$, Mt. (Greenleaf, Rand); - Western Mt.; White Beach, Great Peacé Pond (Rand). Cone ( 2 sy. T

A. canina, I. B. B. (m. in the ?r.)

Infrequent. Sargent Mt. (Greenleaf, Rand).

2xalforin:

\section{CINNA, L. WOOD REed Grass.}

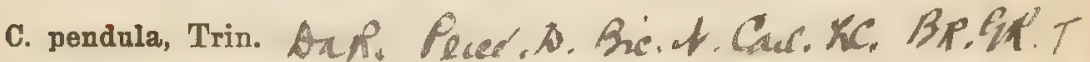

Damp woods. Cold Brook; Little Harbor Brook valley; $\mathrm{d} / \mathrm{C}$; Beech Mt. Notch (Rand); - Seal Harbor (Redfield). A very
robust form, head of Beech Mt. Notch (Rand). "द

Calamagrostis, Adans. Reed Bent Grass.

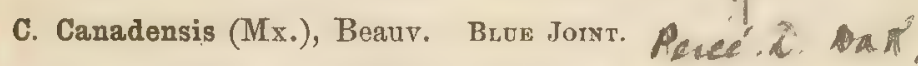

Common in moist or wet ground from sea level to mountain $C, B, 10$,

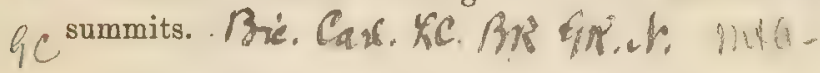

Pdu. Q

DESChampsia, Beauv. Hair Grass.

D. flexuosa (L.), Trin.

Common in dry places, especially on hills and mountains. $C, N d u h$.

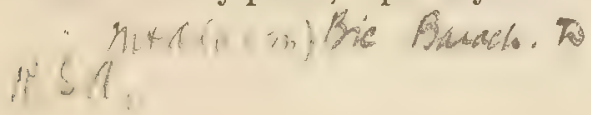

5 


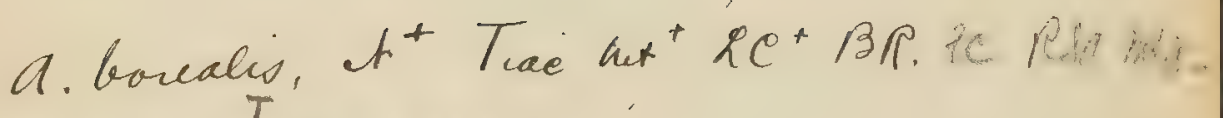
A. Iurm gemale mith-

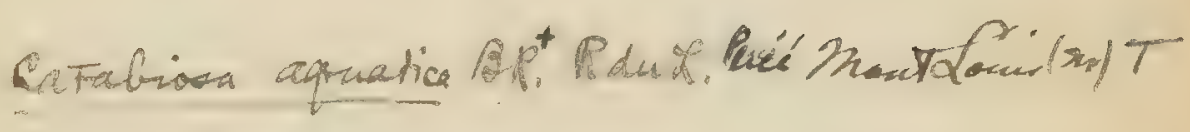

Thiens tanatiw. 5 .

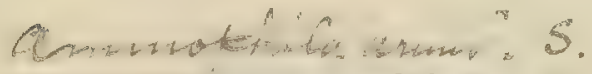

Cal, Seriburi, Le $e^{F}$, RSA $^{+}$

cionsonfic nit $a \pm$

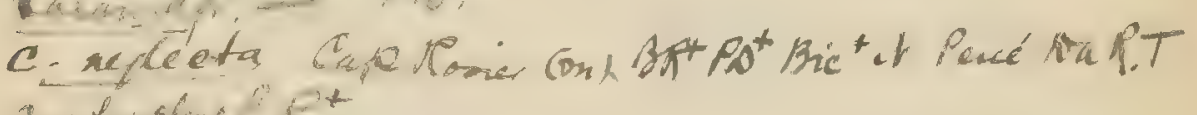

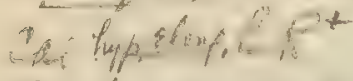

Cal perforaraviour biet

Cal neg toncalis. nexat.

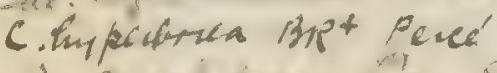

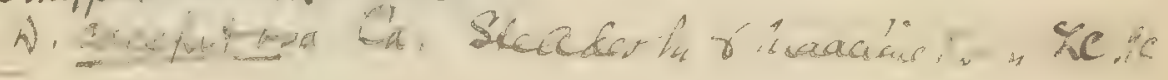

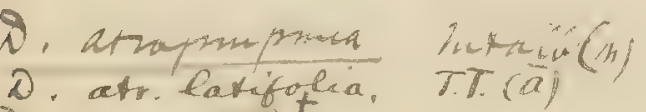

$D_{1}$. atr. Lavif

D. Cuesp-alfina hitd- 
Zues pating $5,4, y k$

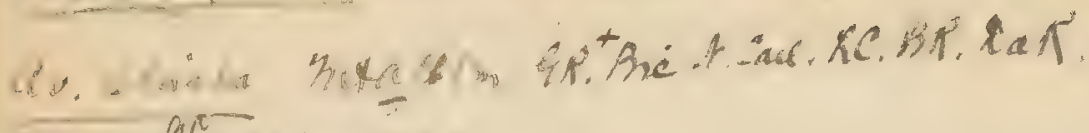

A. . ot ac allicanisinta:

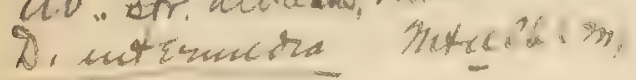

Rinatimo. Ca. Aat

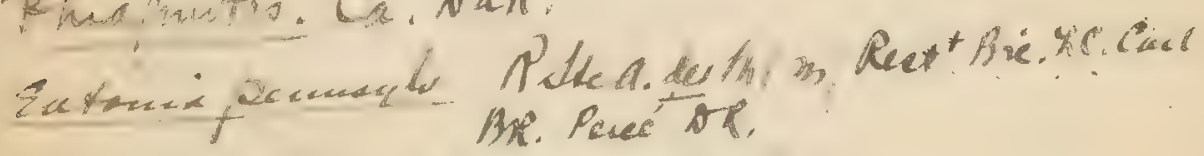

Distichion snantina st.

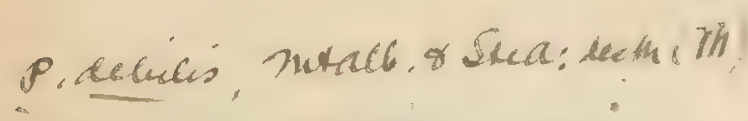

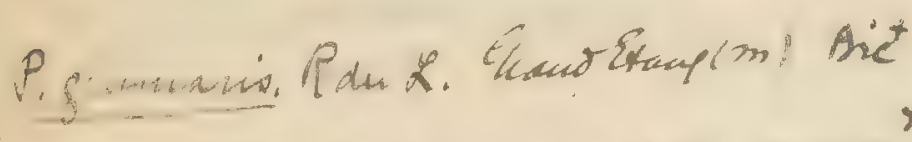

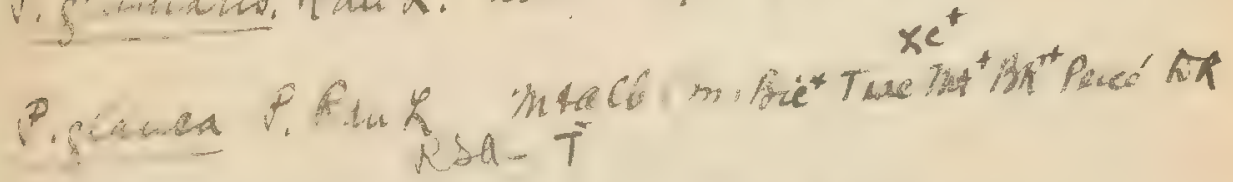

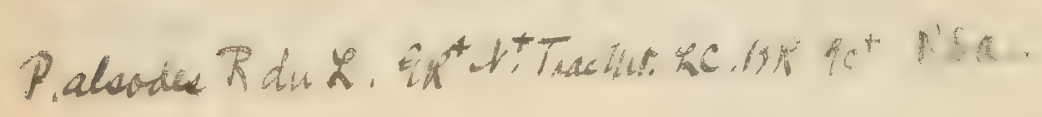

P. S.

Pao tivinutio. S Pacé

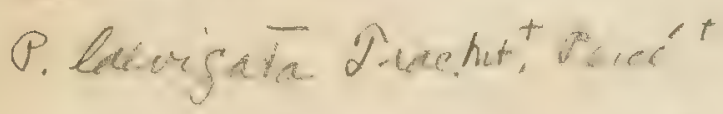

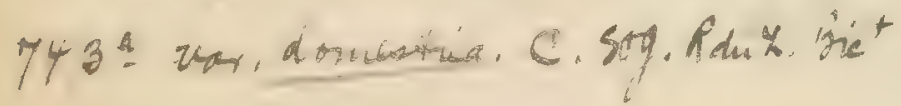

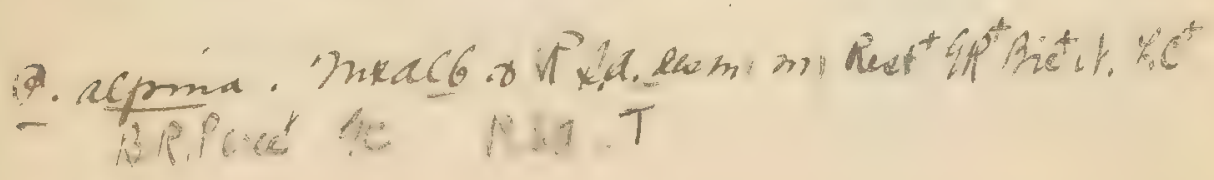




\section{Pesci HC e TRISETUM, Pers.}

$7 / 36$

T. subspicatum (L.), Beaur., var. molle (Mx.), Gray.

$$
\text { Danthonia, DC. WILD OAT Grass. }
$$
137
D. spicata (L.), Beauv.

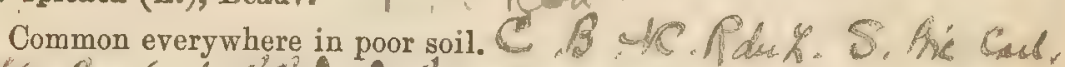

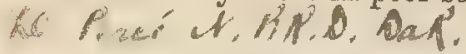
DACTYLIS, L. ORChaRd Grass.

D. GI OMERAtA, L.

Sparingly introduced. Northeast Harbor; "Fox Dens,", Southwest Harbor; Somesville (Rand). Naturalized from Europe.

POA, L. Spear Grass. Meadow Grass.

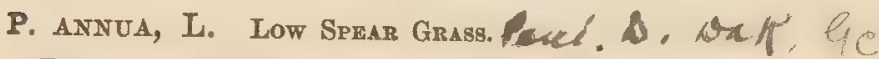

Roadsides and cultivated grounds. Bar Harbor; Southwest $C^{2}$ is of Harbor; Northeast Harbor; Somesville, and elsewhere. Intro $\& 4$. duced, and becoming common. Naturalized from Europe. Cuet AP,

P. compressa, L. Wire Grass. Pewé

Frequent in dry soil, or rocky places. Appearing indigenons $8 t_{\mathrm{H}} \mathrm{d}$ in many places on the Island, but doubtless naturalized from 6 Europe. cone $(x$, Cu: BR.

P. nemoralis, L. Peseś

Somesville (Rand). " dic AC. MPt T

P. serotina, Ehrh. FALSE Red-Top.

Common and very variable. This species runs gradually into $P$. nemoralis; specimens from Bubble Pond (Rand) can hardly be distinguished. A large form with ample panicle ( $P$. fertilis, Host), Somes Pond, at outlet, in water (Rand).

P. pratensis, I. Kentucky Buve Grass.

Common. Perhaps indigenous, but mostly naturalized from

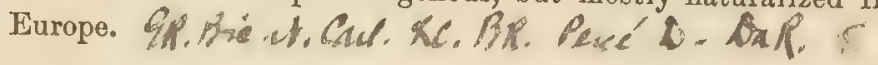




\section{GLYCeria, R. Br. MANNA Grass.}

G. Canadensis (Mx.), Trin.

Common in wet places.

Ratrlesnake Grass. Job's Tears.

\section{G. 1axa, Scribner.}

A coarse leafy grass, $2^{\circ}-4^{\circ}$ high, with a diffuse ample panicle and oblong, somewhat turgid spikelets. Sheaths scabrous, the lower exceeding the internodes. Ligule about $1^{\prime \prime}$ long, thin, lacerate. Leaves $8^{\prime}-15^{\prime}$ long, $3^{\prime \prime}-4^{\prime \prime}$ wide, very rough-scabrous, both sides tapering to a sharp point or the lower ones abruptly sharp-pointed. Panicle $7^{\prime}-9^{\prime}$ long, the main axis and branches strongly scabrous, lower branches in twos or threes, the upper solitary, the longer and usually widely spreading lower ones $3^{\prime}-5^{\prime}$ long. Spikelets oblong or broadly ovate, 3-5-flowered, about $2^{\prime \prime}$ long, much exceeding the pedicels, and from $1^{\prime \prime}-1 \frac{1}{2}{ }^{\prime \prime}$ wide. Empty glumes unequal, scarious-margined, the larger second glume about one half the length of the first floret. Flowering glumes rounded on the back, $1^{\prime \prime}-1 \frac{1}{1}^{\prime \prime}$ long, broadly obovate, obtuse, with a narrow scarious margin above, 7 -nerved, nerves not prominent. Palea nearly equalling the glume, the keels smooth, strongly curved above. Closely allied to $G$. Canadensis, but the smaller spikelets are green or purple tinged, and the more obtuse floral glume scarcely exceeds the narrower palea. F. Lamson-Scribner in Bull. Torr. Bot. Club, xxi. 37, sub nom. Panicularia laxa, and republished here as above at his desire. Seal Harbor (Redfield); - Somesville (Rand). A form apparently of this species with smaller and imperfectly developed spikelets, Great Cranberry Isle; Seal Harbor (Redfield).

G. obtusa (Muhl.), Trin.

Common in wet grounds about Somesville. Also Southwest Harbor; Sea Wall (Rand).

G. elongata (Torr.), Trin.

Wet woods. Near Beech Hill (Arnold Greene); - Canada Valley; Seal Harbor; Beech Mt. Notch (Rand).

G. nervata (Willd.), Trin.

Common in meadows.

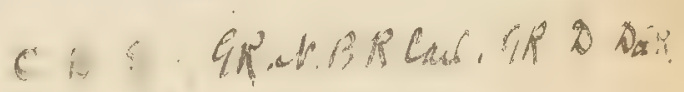

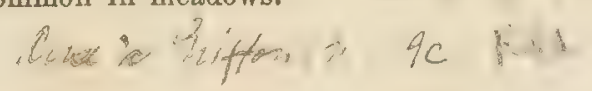




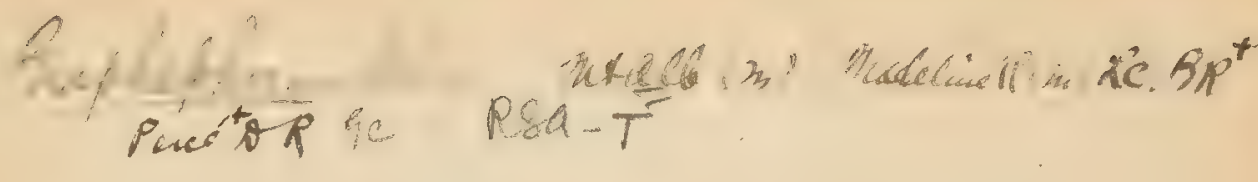

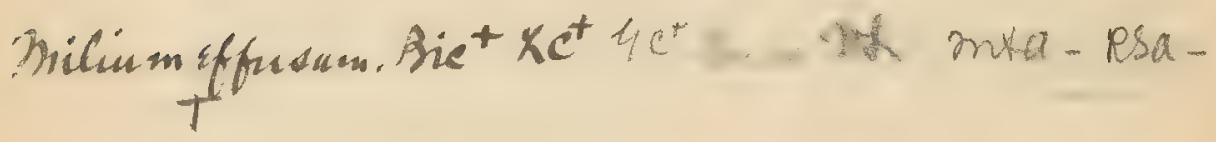

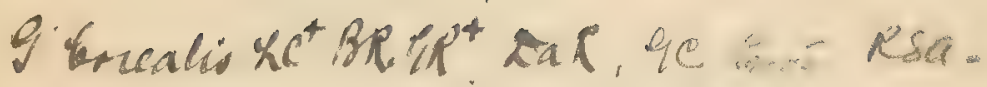


GAvitan of Cur. Pencé

P. tistaros. 3x:

I avose cant

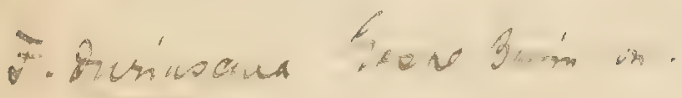

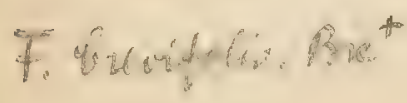


G. pallida (Torr.), Trin. $20 \mathrm{KC}^{+}$

A narrow-leaved form in bog at northern foot of Beech Hill (Rand).

G. grandis, S. Watson. G. aquatica (L.), Sm.

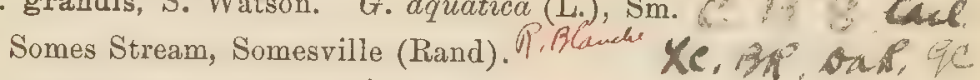

G. fluitans (L.), R. Br. of Ruwer.

Brooksides and ditches; frequent.

\section{PUCCINELLIA, Parl.* (Atropis, Rupr.)}

P. maritima (Huds.), Parl, Atropis maritima (Huds.), Griseb. Glyceria maritima (Huds,), Wahl. SEa Spear Grass.

Rare. Shore, Somes Harbor (Redfield). A puzzling form, perhaps a hybrid between this species and $P$. distans, Norwood Cove (M. L. Fernald). Geses in Cul T is

Var. (?) minor, S. Watson. Atropis maritima, var. Nutkaensis (Presl), Scribner. Atropis angustata (R. Br.), Griseb.

Puccinellia angustata (R. Br.). Glyceria angustata (R. P

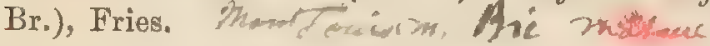

Sea beaches. Somesville; Orens; Thomas Bay; Southwest Harbor; Mt. Desert Narrows, and elsewhere. "This form is distinguished from $P$. maritima by its smaller and weaker habit, and by having the keels of the palea smooth below and only very minutely scabrous above. In $P$. maritima the keels of the palea are strongly fringed nearly or quite to the base." F. LamsonScribner. More recent examination shows this form to be specifically distiuct, and should bear the name in the Manual of $P$. angustata (R. Br.).

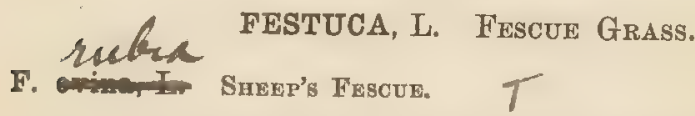

Common especially on or near the shore. Also on Cranberry Isles. All specimens from Mt. Desert and vicinity are referred, however, to $F$. rubra, L., by Prof F. I Jamson-Scribner, which

* Better considered as a sub-genus of Glyceria. Areé

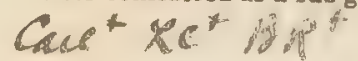


he considers without doubt a distinct species from $F$. ovina. Specimens from High Head, Great Cranberry Isle, Little Cranberry Isle, and 'Thompson Island, may be referred to var. genuina, Hack. A specimen from Indian Point road, Somesville, appears to be var. fallax, Hack.

"In $F$. rubro the leaves of the culm and sterile shoots are similar, the ligules in the latter are not auriculate, and the shoots themselves are extra-vaginal; i. e. the buds of the branches at the base of the culm burst through the base of the leaf sheath in the axil of which they are formed. In F. ovina the leaves of the flowering culms and sterile shoots are unlike, the ligules on the latter are auriculate and the shoots themselves are intra-vaginal; i. e. the buds in the lower leaf axils grow up out of the sheaths and do not break through them below. $F$. ovina is strictly tufted, while $F \cdot$ mubra extends more or less by rootstocks." F. Lamson-Scribner.

F. DURTUscula, L. F. ovina, L., var. duriuscula, Koch. Gray, Man., 6th ed.

Rare: Near Hulls Cove (Rand). Naturalized from Europe.

F. ELATYOR, L.

Fields and roadsides; common. Northeast Harbor; South- $C$, $C$ west Harbor; Bar Harbor; Somesville; Seal Harbor; High Head. Naturalized from Europe.

Var. Pratensis (Huds.), Gray.

Fields. Seal Harbor; Southwest Harbor; Somesville; High Head, and elsewhere. Naturalized from Europe.

BROMUS, L. Brome Grass.

B. ciliatus, L. $2 C^{+}, A R$. AR. $A R, \&, A c$, $f$,

Frequent in rocky woods and low ground. Variable.

AGROPYRUM, Gærtn. (Triticum, L.) False Wheat.

A. repens (L.), Beauv. Triticum repens; L. Qurtcr Grass. Witch Grass. Ais, \& T

Fields, waysides, and shores; common and very variable.

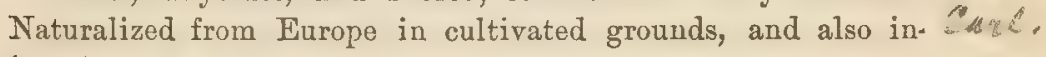

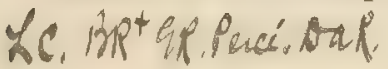




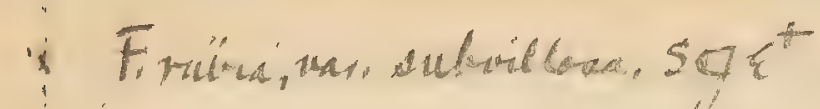

- P. deabulla málolom

Br.ciliarns vir. Caveriglomins 'xct Bipeatinus B.

Br.

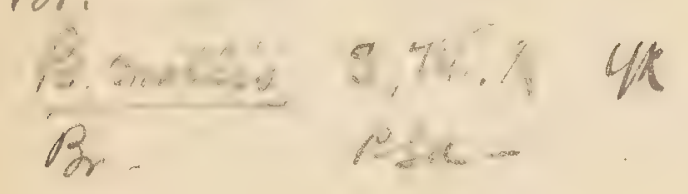




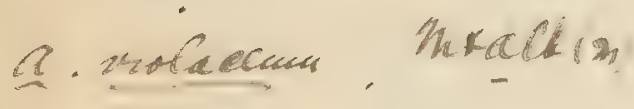

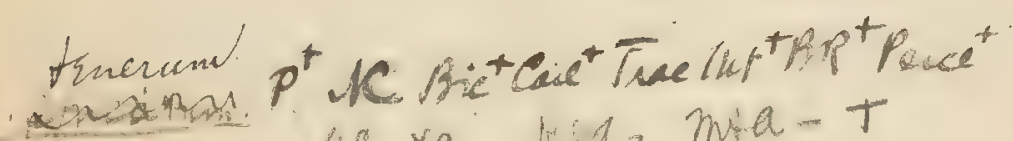
matane la, xc M:A, Mia-T

Drati, R. RS . 
digenous. The Island forms appear for the most part to be indigenous northern and coast forms, and abound on beaches and on banks by the shore.

Var. glaucum (Desf.), Boiss. Triticum repens, L., var. intermedium, Fries.

A glaucous, rigid, maritime form, with large crowded spikelets and glumes blunt or mucronate. Seal Harbor (Redfield); Northeast Harbor; Southwest Harbor, etc. (Rand).

Var. pilosum, Scribner.

Upper surface of leaves pilose, rhachis of spike pubescent to 12 . hirsute, flowering glumes awnless or short cuspidate pointed. Southwest Harbor (Rand). This, however, may be the same as var. agreste, Anders.

Other well marked forms are numerous; but it seems impossible to identify them with any certainty without a careful comparison with authentic specimens in European herbaria.

A. caninum (L.), Roem. \& Schultes. Triticum caninum, L. Nall, NA. Ahe Rare. Field near the head of Northeast Harbor (Rand). 1het, AC. Aft Naturalized from Europe, and also indigenous. Probably introduced on the Island. Whit

\section{HORDEUM, L. BARLEY.}

H. jubatum, L. Squirred-Tail Grass.

Common on the coast. Bre, di, Gab MA, Ax R

ELYMUS, L. Wild Rye. LyMe Grass.

E. Virginicus, L. AaR. T.

Common on the coast.

E. mollis, Trin.

Muddy or sandy shores on the coast; frequent. Considered by many authors as identical with $\boldsymbol{E}$. arenarius, L., which occurs on the Pacific coast. He. Brs. D. T 
SERIES II. CRYPTOGAMIA; FLOWERLESS PLANTS.

\section{Crass I. PTERIDOPHYTA.}

EQUISETACE E. Horsetair Family.

EQUisetuM, L. Horsetail. Scouring Rush.

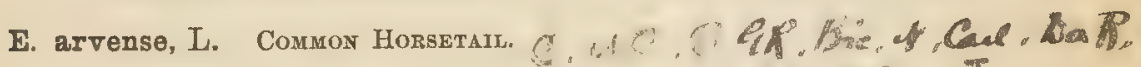

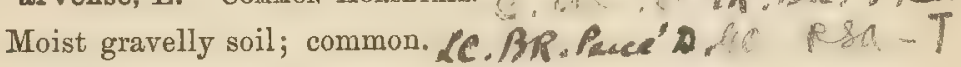

E. sylvaticum, L. Atheficul. D.

Wet banks and shady places; frequent. C r W , RS

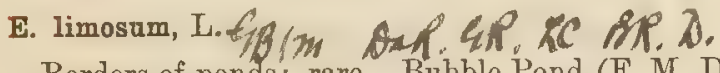

Borders of ponds; rare. Bubble Pond (F. M. Day, Redfield); $C$, a

- Great Pond (Rand).

FILICES, Ferns.

POLYPODIUM, L. POLYPODY.

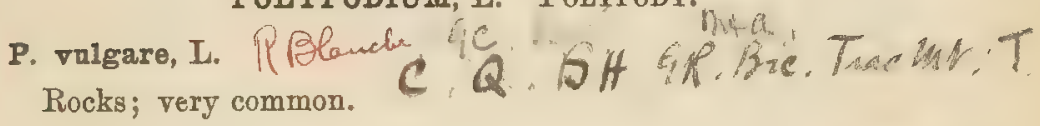

PTERIS, I. BRAKE. PiC

P. aquilina, $\mathrm{L}$.

Dry soil; very common.

ASPLENIUM, L. SPLEENWORT.

A. Filiz-fœmina (L.), Bernh. Lady FERN. $\bar{D}, 4 / 3$

Damp shady places; frequent, and very variable.

Var. angustatum (Willd.), D. C. Eaton. Var. Michauxi, Mett.

Fronds $1^{\circ}-3^{\circ}$ high, rather rigid, narrow in outline, nearly. bipinnate; pinnæ obliquely ascending or curved upwards, narrowly lanceolate; segments oblong, crowded, crenated or serrate; 


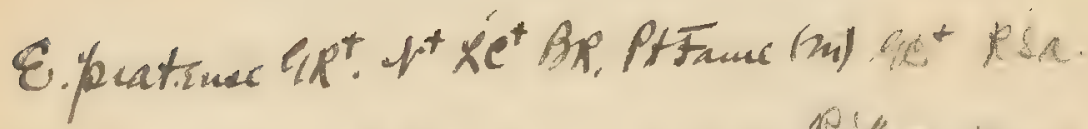

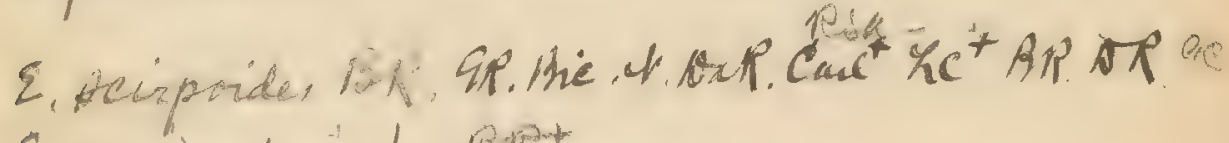

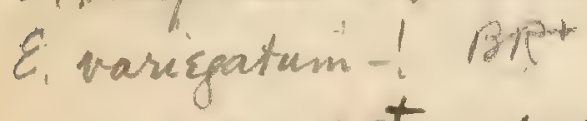

2. palustre ARt Cact oR ouR. PiA.

$\therefore$ E. variggaturw Ant Percé Ax. eng (m)

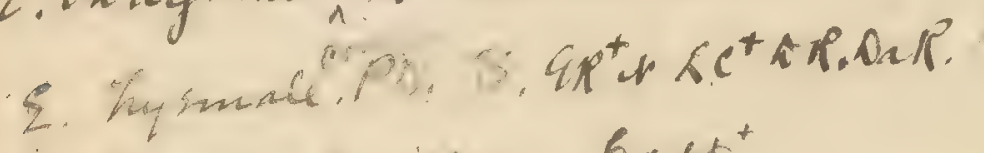

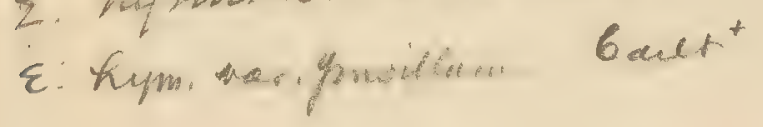

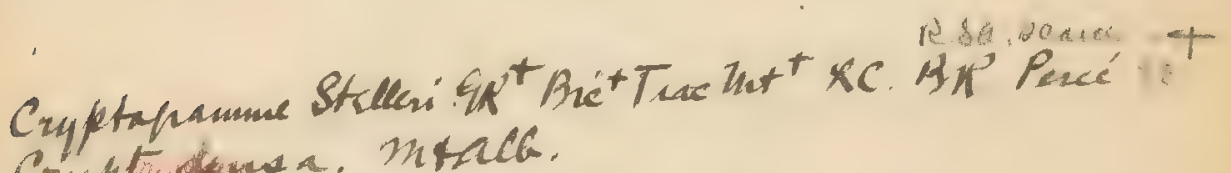

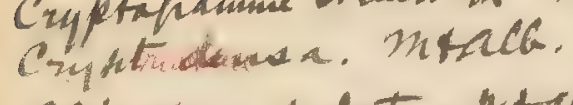

ariantume peratum. nitrla(m) Eit.

a.p.var.alecticum, $\%$ is.

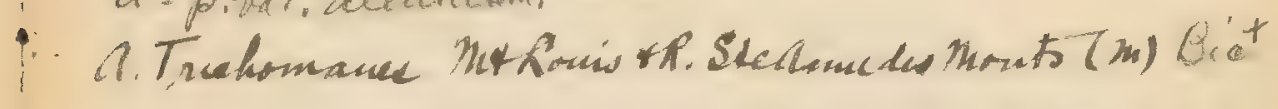

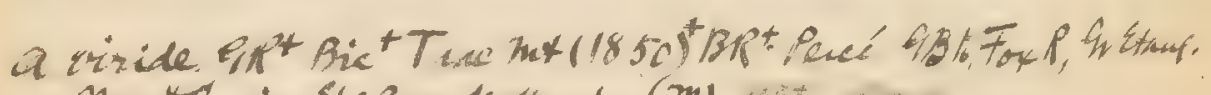

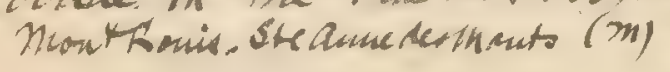

a. thetpenterides the ic 
P. calcarca PRt 
sori usually abundant, straight or curved. Eaton, Ferns N. A. 227. Woods; infrequent. Salisbury Cove (Clara L. Walley).

Var. exile, D. C. Eaton.

Fronds $3^{\prime}-6^{\prime}$ high, lanceolate, pinnate; pinnæ oblong-lanceolate, deeply cut into oblong laciniæ which are two- to threetoothed at the end. Eaton, Ferns N. A. 227. Woods; rare. Breakneck road (Clara L. Walley).

\section{phegopteris, Fée. Beech Fern.}

P. polypodioides, Fée. Polypodium Phegopteris, L. A. 19 , qC

Common in damp woods. A form closely approaching $P$. hexT agonoptera, Fée, in woods, Seal Harbor (Redfield). A form with the main rhachis forked at the apex, woods, head of The Barcelona meadow (Rand). IP. N Am. Are t. KC. Pecé

P. Dryopteris (L.), Fée. ThA- $A A^{3} 4 B$.

T Damp woods and shaded rocky places; common. Ras t the. XC. Peaci to

\section{ASPIDIUM, Swz. Shield FerN.}

\section{A. Thelypteris (L.), Swz.}

Damp ground; not uncommon. Seal Harbor; Long Pond meadows; Sutton Island; Great Cranberry Isle; Baker Island. (Redfield); - on Somes Stream (R. \& R.).Caŕ. BR.

\section{A. Noveboracense (L.), Swz.}

Damp woods; frequent. Seal Harbor; Great Cranberry Isle, $C A$ etc. (Redfield); - Breakneck road (Clara L. Walley); - "Mt. Desert" (F. M. Day).

A. spinulosum (Retz), Swz.

Damp woods; not uncommon. Breakneck road (Clara I. Sif, Walley): - Seal Harbor; Orens (Redfield).

Var. intermedium (Muhl.), D. C. Eaton.

1C 5,9 ,

Woods; common. 


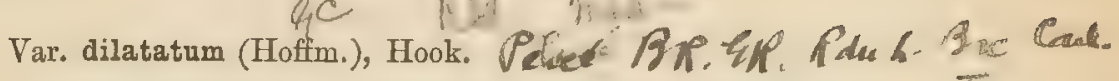

Woods; infrequent. . Breakneck road (Clara L. Walley); west of Southwest Harbor (M. L. Fernald). A dwarf form in woods, Breakneck road (Clara L. Walley).

A. cristatum (L.), Swz.

Wet ground; frequent.

C.AC Car. Hp. Ant.

Var. Clintonianum, D. C. Eaton.

Rare. Near Breakneck road (Clara L. Walley).

A. marginale (L.), Swz.

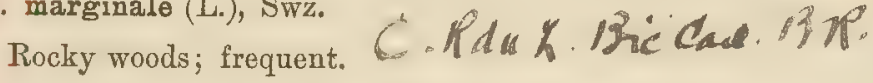

A. acrostichoides (Mx.), Swz. Christmas Ferk.

Deep rocky woods; frequent.

CYSTOPTERIS, Bernh. BLADDER FERN.

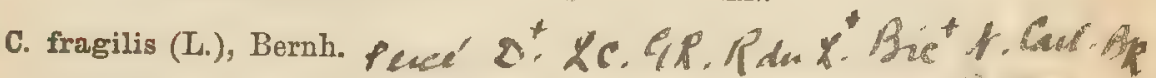
Rare. Caves, Barr Hill (Redfield); - wet cliffs, West Branch of Hadlock Brook (Rand). GC inpa-T

ONOCLEA, L. SeNsitive FERN.

o. sensibilis, L.

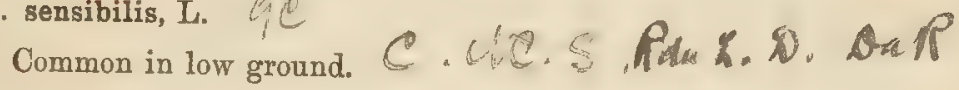

WOODSIA, R. Br.

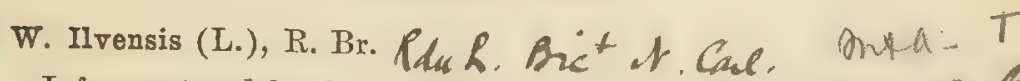

Infrequent and local. Dog Mt. (Rand); - Flying Mt. (An- Q $4 p^{+}$ nie S. Downs, H. C. Jones, and others); - Beech Cliff (Annie S. Downs, R. \& R.).

\section{DICKSONIA, L'Hér.}

D. pilosiuscula, Willd. Common everywhere.

D. punctilobula (Mx.), Gray. $\Leftrightarrow$. 


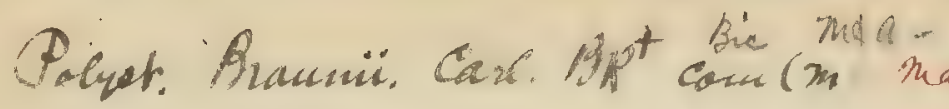

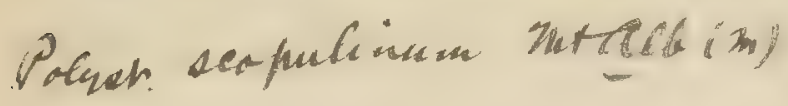

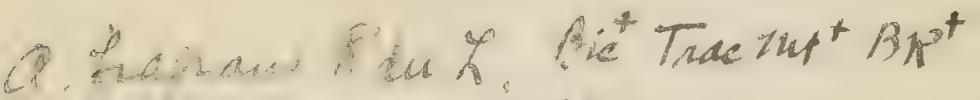

R.STA.An, TeS.R(m) inta-

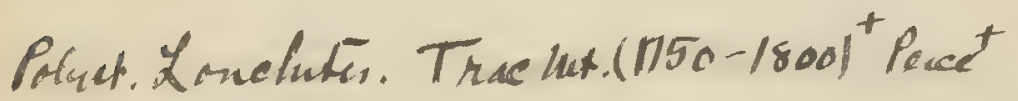

A. Filix-thas Pesee't Joy R(m)

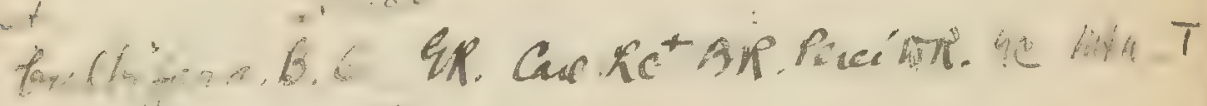
C. montina mtall $(m)$

C. $9 \pi$

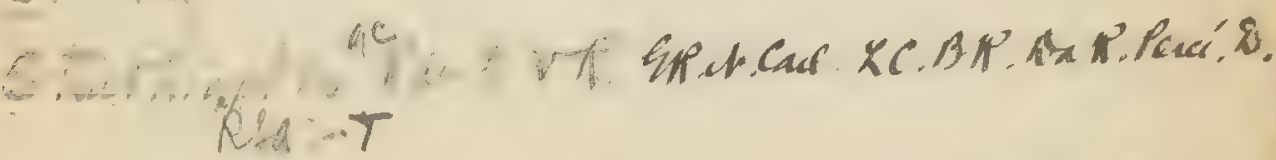

Rin $\rightarrow$

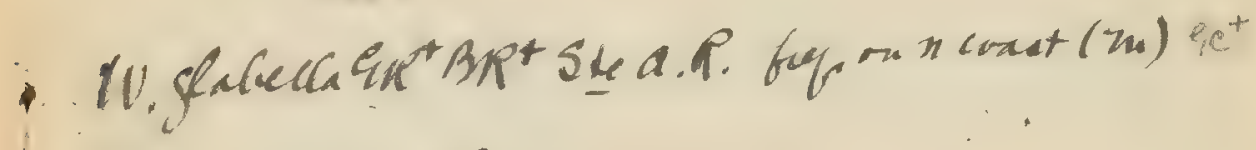

- Hi: aregana $B_{i i^{*}}$

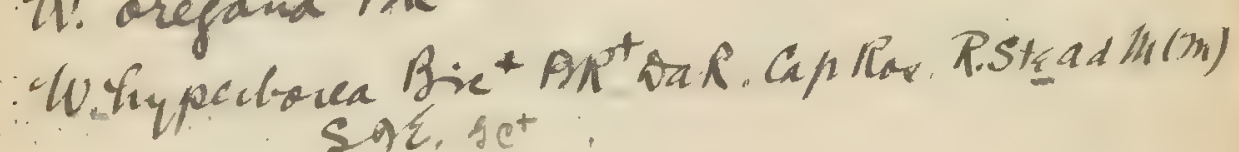
Sye. get $^{+}$.

W. focopuina $T^{+}$

firomatia ripisica. Ele (m) 


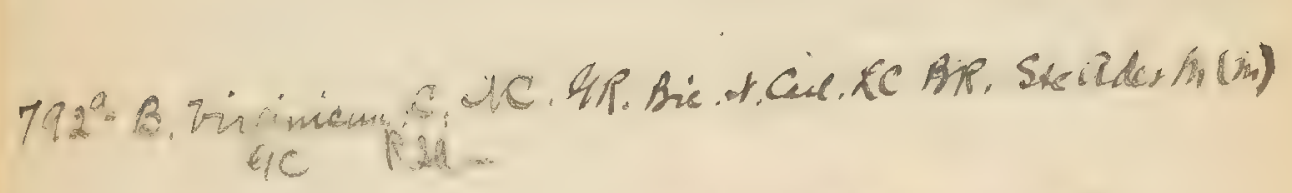

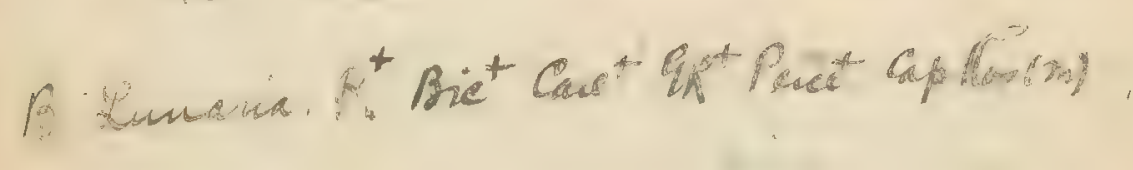


OSMUNDA, L. Flowering Fern.

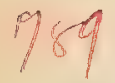

790

791

\section{0. regalis, L. Flowering FerN.}

Swamps and wet meadows; common.

\section{Claytoniana, L. Internupted Flowering Fern.}

Common in low ground. A form with upper pinnæ of sterile frond partly fertile, Bar Harbor (Mary Minot). A form with middle pinno of fertile frond partly sterile, and undeveloped, Az f, Seal Harbor (Redfield). Futh -

0. cinnamomea, I. Cinnamon Fern.

Common in damp ground everywhere.
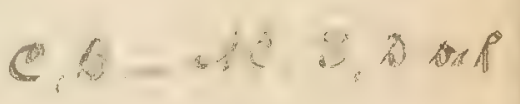

\section{OPHIOGLOSSACEAE. Adder's Tongue Family.}

\section{BOTRYCHIUM, SWz, MOONWORT.}

B. simplex, Hitcheock. Hict $\mathrm{H}^{+} \mathrm{fC}^{+} \mathrm{F}_{\mathrm{C} . . .}+\mathrm{T}$

Rare. High Head (Rand, Redfield \& Faxon); - Beech Cliff (Rand).

B. matricariæfolium, A. Br.

Infrequent. Jordan Pond road (Harriet A. Hill); 一 field, $\rho d$ Northwest Cove; Cold Brook; Great Cranberry Isle (Rand).

B. ternatum (Thunḅ.), Swz.

Somewhat frequent in low fields and pastures; occasionally in woods. Seal Harbor; Long Pond meadows (Redfield); - Sutton Island; Sawyer Cove (Harriet A. Hill); - Duck Brook road (Clara L. Walley); - woods, north of Jordan Pond (Theodore G. White); - meadow, head of Northeast Creek; Southwest Valley road (Rand).

Var. intermedium, D. C. Eaton.

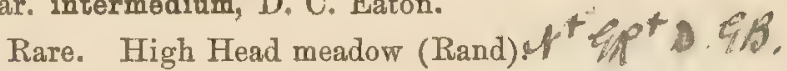
Var. obliquum (Muhl.), Milde.

Not uncommon. Duck Brook road (Clara L. Walley); meadow, head of Northeast Creek; Southwest Harbor (Rand);

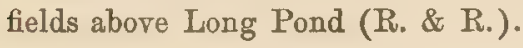


Var. dissectum (Spreng.), Milde.

Frequent. Duck Brook road (Clara L. Walley);-meadow, head of Northeast Creek; High Head meadow; fields, Clark Point, Southwest Harbor (Rand); - fields above Long Pond (Redfield); - Sawyer Cove (Harriet A. Hill).

OPHIOGLOSSUM, L. ADDER's TONGUE.

0. vulgatum, $\mathrm{L}$.

Rare. Wet field, head of Southwest Harbor (Annie S. Downs).

LYCOPODIACEA. Club Moss Family.

LYCOPOdiUM, L. Club Moss.

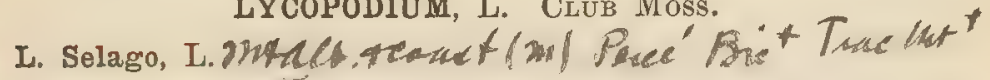

Rare. Sargent Mt. (H. C. Jones, Rand, Redfield).

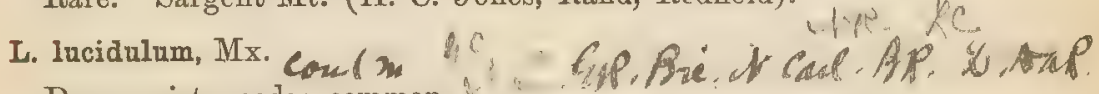
Deep moist woods; common.

I. inundatum, L.

Bogs' and wet places; frequent. Cedar Swamp; Southwest Harbor; Sea Wall Swamp; Aunt Bettys Pond; Ripples Pond; Gilmore Meadow; Great Cranberry Isle, etc. (Rand); - bog, Hadlock Upper Pond, etc. (Redfield).

Var. Bigelovii, Tuck.

Bogs; uncommon. Border of Upper Breakneck Pond; border of Aunt Bettys Pond (Rand).

L. annotinum, L. $\operatorname{com}(2 n)$ (ic $R(1-m \times d$,

Woods and damp thickets; frequent. Also Sargent Mt.

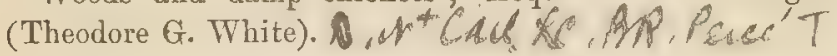

L. obscurum, L.

Rare. Roadside between Somesville and Southwest Harbor, near Canada Valley (Rand).

Vax. dendroideum (Mx.), D. C. Eaton. Ground Pine. C $\mathbf{B} y^{2} \mathrm{Ap}^{4}$.

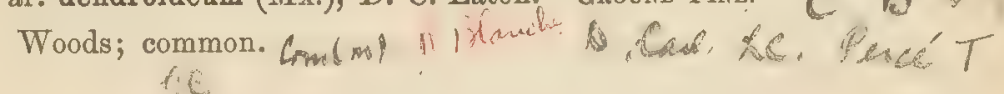


c

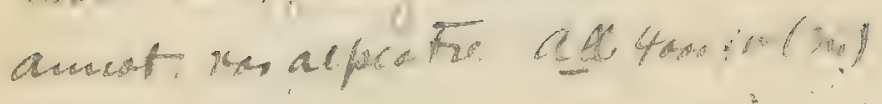




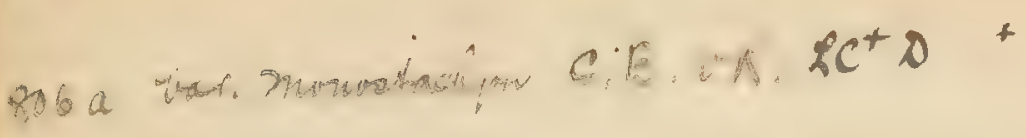

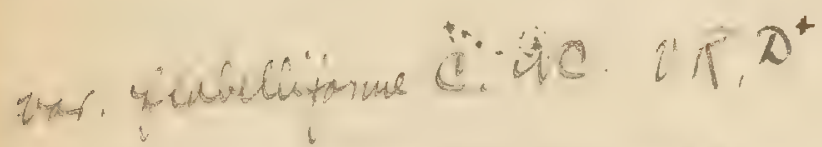

R. sitchense thtept 0 . $4 k$ mea -

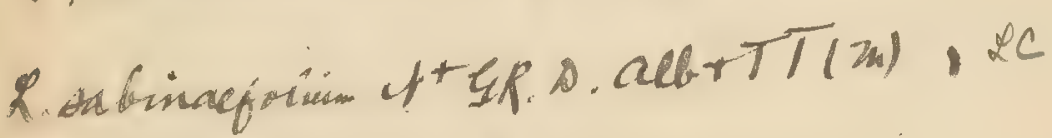

L. alponinar mia.

S. spinosa. $4 R^{+} R C^{+} B R^{+}$IR Pexé gradr R.SLedd moni mial. 


\section{KSa-}

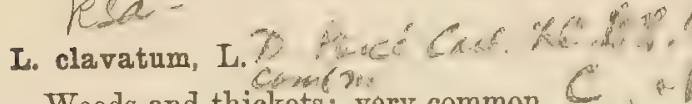

Woods and thickets; very common.

I. complanatum, L. Trailing Christmas Gremen.

Dry woods and thickets; common. A tof

Var. Chamæcyparissus (Braun), D. C. Eaton.

Woods and mountain thickets; infrequent. Between Jordan Pond and Eagle Lake (Redfield); - between Jordan Pond and Northeast Harbor (R. \& R.);-Pemetic Mt.; woods, Aunt Bettys Pond (Rand). Vi. Houde

\section{SELAGINELLACE $\not E$.}

SELAGINELLA, Beauv.

S. rupestris (L.), Spring.

Rare and local. Flying Mt. (H. C. Jones, Rand); - Dog Mt. (Elizabeth G. Britton, Rand, E. Faxon).

\section{ISOETES, L. QUILLWORT.}

I. lacustris, L.

Infrequent. Mouth of Deer Brook, Jordan Pond (Redfield); - west shore of Jordan Pond (Rand). A very small form on west shore of Jordan Pond (Rand). A peculiar form, probably of this species, Deer Brook (Rand); - Northwest Arm, Great Pond (Fernald).

I. echinospora, Durieu, var. Braunii (Duxieu), Engelm.

Sandy and gravelly brook courses and pond shores; frequent. Jordan Stream; Deer Brook; shores of Jordan Pond (R. \& R.);

- Somes Stream; Denning Brook (Rand).

I. riparia, Engelm.

Rare. Sornes Stream (George G. Kennedy); - southeast end of Ripples Pond (Rand). A form "not typical I. riparia, and yet quite a departure from I. lacustris," — Ripples Brook (Rand, M. L. Fernald), fide L. M. Underwood. 


\section{Class II. BRYOPHYTA.}

\section{Drvision I. MUSCI; MOSSES.}

\section{Order I. SPHAGNACFAE. Peat Mosses.}

List prepared by Edward I. Rand, assisted greatly by Edwin Faxon and Prof. Daniel C. Eaton, and arranged mainly in accordance with the writings of Dr. Carl Warnstorf, of Neuruppin, Germany.

The plants catalogued in the following list have been collected mostly by Edwin Faxon and Edward L. Rand; the determinations are by Dr. Carl Warnstorf. In view of the great difficulty of finding descriptions of the various species and varieties, it has been thought wise to give freely references to Lesquereux and James's "Mosses of North America," and to Dr. Warnstorf's articles on North American Sphagna, to be found in Coulter's Botanical Gazette, Vol. XV., in the numbers for the months of June, August; September, and October, 1890 , - both of which works can be consulted with little trouble. In cases, furthermore, where descriptions are not there given, they have been either translated or specially prepared for this list by Prof. Eaton and Mr. Faxon. It has not seemed best to include forms and sub-forms herein, since most of them have little value except for the critical student. Very many of them are, however, represented in the Mt. Desert Herbarium. On the other hand, all varieties are given, without regard to the distinctions on which they are founded. The value, however, of most of the so-called color varietieg is very doubtful, aince careful observations seem to prove beyond question that the color of Sphagnum varies greatly with the season. It is even a matter of doubt whether a given plant of any species may not quickly vary through the influence of temporary external conditions, assuming at one time the form of one variety, at another time the form of another. This doubtful value of varieties of Sphagnum furnishes an additional reason for the exclusion of mere forms and sub-forms from a local catalogue of plants. 
$\frac{8}{2}$
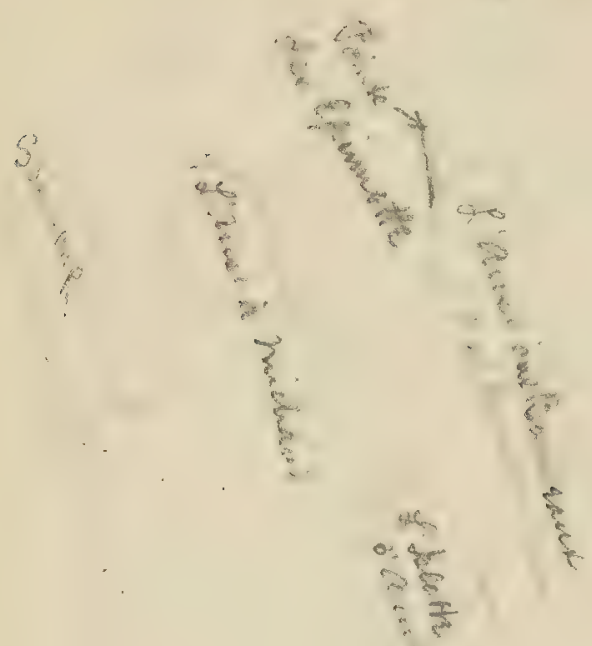

है
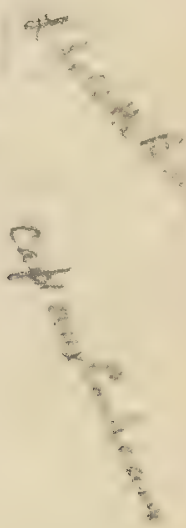


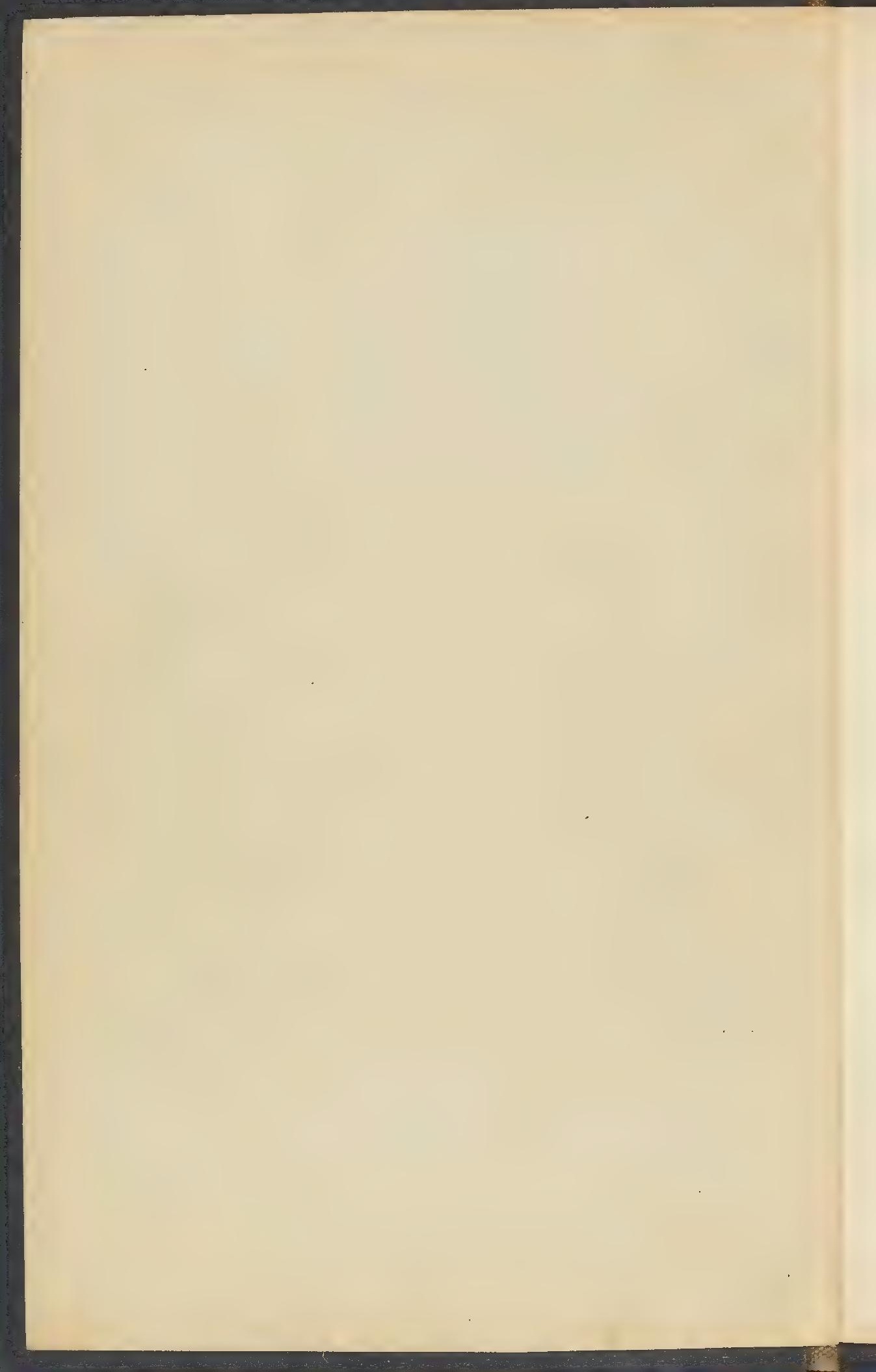




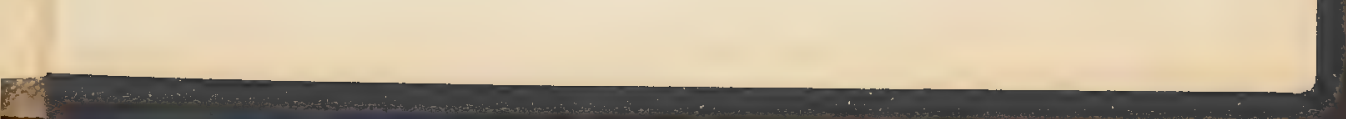




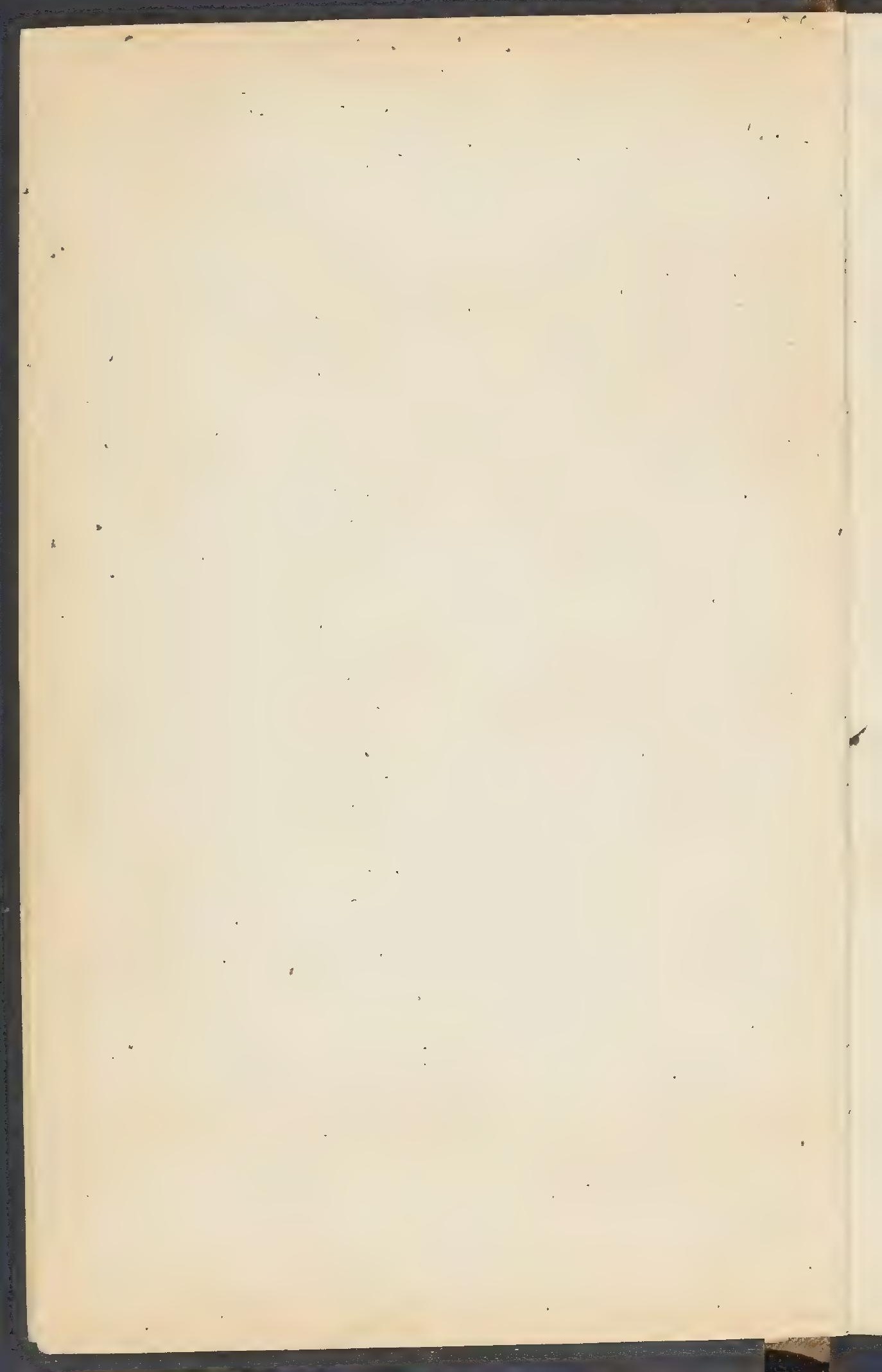





